LBL-36197

\title{
Study of Small Carbon and Semiconductor Clusters Using Negative Ion Threshold Photodetachment Spectroscopy
}

\author{
Caroline Chick Arnold \\ Ph.D. Thesis \\ Department of Chemistry \\ University of California
}

and

Chemical Sciences Division

Lawrence Berkeley Laboratory

University of Califomia

Berkeley, California 94720

Augusi 1994

This work was supported in part by the Director, Office of Energy Research, Office of Basic Energy Sciences, Chemical Sciences Division of the U.S. Department of Energy under Contract No. DE-AC03-76SF00098, and in part by the National Science Foundation. 


\section{Study of Small Carbon and Semiconductor Clusters Using Negative Ion Threshold Photodetachment Spectroscopy}

Copyright (ㄷ) 1994

by Caroline Chick Amold

The Government reserves for itself and others acting on its behalf a royalty free, nonexclusive, irrevocable, world-wide Iicense for govermental purposes to publish, distribute, translate, duplicate, exhibit, and perform any such data coprighted by the contractor.

The U.S. Department of Energy has to right to use this thesis for any purpose whatsoever including the right to reproduce all or any part thereof 


\title{
Study of Small Carbon and Semiconductor Clusters Using Negaive lon Threshold Photodetachment Spectroscopy
}

\author{
by \\ Caroline Chick Amold \\ Doctor of Philosophy in Chemistry \\ University of Califomia, Berkeley \\ Professor Daniel M. Neumark, Chair
}

The bonding and electronics of several small carton and semiconductor clusters containing less than ten atoms are probed using negative ion threshold photodetachment (zero electron kinetic energy, or ZEKE spectroscopy. ZEKE spectroscopy is a particularly advantageous technique for small cluster study, as it combines mass selection with good spectroscopic resolution. The ground and low-lying electronic states of small clusters in general can be accessed by detaching an electron from the ground anion state. The clusters studied using this technique and described in this work are $\mathrm{C}_{6}{ }^{-} / \mathrm{C}_{6}, \mathrm{Si}_{n}{ }^{-} / \mathrm{Si}_{n}(\mathrm{n}=2,3,4), \mathrm{Ge}_{2}-1 \mathrm{Ge}_{2}, \mathrm{In}_{2} \mathrm{P}^{-}$ $\left\|n_{2} \mathrm{P}, \operatorname{In} \mathrm{P}_{2}-/\right\| n \mathrm{P}_{2}$, and $\mathrm{Ga}_{2} \mathrm{As}$. The total photodetachment cross sections of several other small carbon clusters and the ZEKE spectrum of the $l: \mathrm{CH}_{3} l \mathrm{~S}_{\mathrm{N}} 2$ reaction complex are also presented to illustrate the versatility of the experimental apparatus.

Clusters with so few atoms do not exhibit bulk properties. However, each specie exhibits bonding properties that relate to the type of bonding found in the bulk. $\mathrm{C}_{6}$, as has been predicted, exhibits a linear cumulenic structure, where double bonds connect all six carbon atoms. This double bonding reflects how important $\pi$ bonding is in certain phases of pure carbon (graphite and fullerenes). The symmetric stretch frequencies observed in the $\mathrm{C}_{6}^{-}$ spectra, however, are in poor agreement with the calculated values. Also observed as sharp structure in total photodetachment cross section scans was an excited anion state bound by only $-40 \mathrm{~cm}^{-1}$ relative to the detachment continuum. This excited anion state appears to be a 
valence bound state, possible because of the high electron affinity of $\mathrm{C}_{6}$, and the open shell of the anion.

The small silicon clusters differ substantially from the carbon clusters in that $\pi$ bonding does not in general occur, except for in the dimer. These clusters are much more congested with clase-lying electronic states. For $\mathrm{Si}_{2}$, transitions from two close-lying anion states to five neutral strtes are observed. The trimer ground state is still not known, although we observe transitions to a $D_{3 h}$ neutral from a pseudorotating anion. The $\mathrm{Si}_{4}{ }^{-} \mathrm{ZEKE}$ spectrum, in addition to showing extensive vibrational structure in the first excited neutral state, gives evidence that the close-lying excited electronic states undergo vibronic coupling. The ZEKE spectrum of $\mathrm{Ge}_{2}$ reveals the electronic similarity between the two Group IV dimers, although relativistic effects are more dramatic in $\mathrm{Ge}_{2}$.

Snall group III-V mixed clusters represent a different class of small clusters; more structural isomers are available in a binary system, and the bonding between substituent atoms may have some ionicity involved. From the ZEKE spectra of $\ln _{2} \mathrm{P}^{-}$and $\ln \mathrm{P}_{2}{ }^{-}$, we are able to assign the neutral ground states based on molecular orbital arguments and the atomic motion suggested by the vibrational frequencies observed in the spectra. These two open shell species exhibit interesting trends in vibrational frequencies between the anion and neutral ground states, and, in the case of $\operatorname{lnP}_{2}$, the first excited state. The photoelectron spectra of $\mathrm{Ga}_{2} \mathrm{As}^{-}$and $\mathrm{In}_{2} \mathrm{P}^{-}$ reflect remarkable electronic similanity between the two species, but the respective ZEKE spectra show that the actual bonding properties of the molecular ortitals are in fact different.

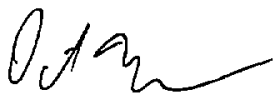


Dedicated to my parents (Mock and Jock) and young Aurelien 


\section{Table of Contents}

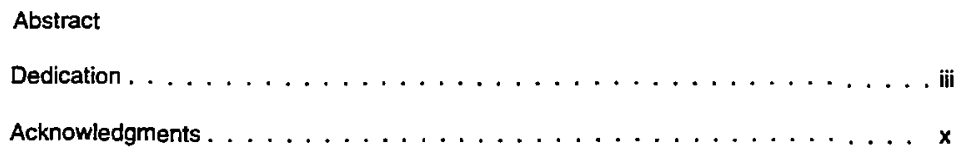

Chapter 1. Introduction . . . . . . . . . . . . . . . . . . . 1

I. Principles of negative ion threshold photodetachment (zero electron

kinetic energy, or ZEKE) spectroscopy . . . . . . . . . 4

II. The Wigner threshold law, and how it applies to polyatomics . . . . . 8

III. Application of negative ion photodetachment techniques toward the characterization of small carbon and semiccnductor clusters . . 10

\section{Chapter 2. Experimenial modifications to the threshold photodetachment} spectrometer ............................. 21

I. The cluster source . . . . . . . . . . . . . . . . 22

A. Piezoelectric molecular beam valve . . . . . . . . . 24

B. Liquid nitrogen-cooled clustering channel . . . . . . . . . 25

II. Deflectors added to the mass spectrometer . . . . . . . . . . . 26

III. Improvements to the detector region . . . . . . . . . . . 27

A. Total photodetachment cross section windows . . . . . . . . 28

B. Five-element Einzel lens . . . . . . . . . . . . . . 29

c. Improved magnetic shielding . . . . . . . . . . 29 
Chapter 3. Study of $C_{6}$ and $C_{6}{ }^{-}$using threshold photodetachment spectroscopy and autodetachment spectroscopy ....................... 37

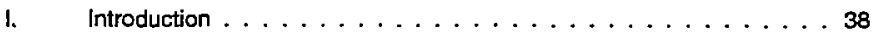

II. Experimental .......................4 4 1

III. Results. ...................... 44

IV. Analysis and Discussion ................ 4 B

A. Threshold photodetachment spectrum ............ 48

1. Peak assignments ................ 48

2. $c_{6}$ force constants ................ 49

3. $\mathrm{C}_{6}-\mathrm{C}_{6}$ geometry change $\ldots \ldots \ldots \ldots . \ldots . \ldots . . . . .52$

4. Comparison to the $\mathrm{C}_{5}{ }^{-}$ihreshold photodetachment spectrum . 54

B. Partially discriminated and total photodetachment cross section spectra . . . . . . . . . . . . . 54

1. Peak assignments . . . . . . . . . . . . . . 54

2. Autodetachment mechanism and autodetaching state lifetimes ................. 60

3. Nature of the excited anion state . . . . . . . . . . 63

v. Conclusion ....................... 65

Chapter 4. Structure observed in the total photodetachment cross sections of $c_{n}{ }^{-}(n=4,6-9) \ldots \ldots \ldots \ldots \ldots$

I. Introduction ..................... 89

II. Experimental ..................... 9 1

III. Results ....................... 92 
IV. Analysis $\ldots \ldots \ldots \ldots \ldots \ldots \ldots \ldots$

v. Conctusions $\ldots \ldots \ldots \ldots \ldots \ldots \ldots$

Chapter 5. Reassignment of the $\mathrm{Sit}_{2}{ }^{-}$photodetachment spectra . . . . . . . . 111

Chapter 6. Threshold photodetachment (ZEKE) spectroscopy of $\mathbf{S i}_{3}{ }^{-} \ldots \ldots \ldots 121$

I. Introduction . . . . . . . . . . . . . 122

II. Experimental . . . . . . . . . . . . . . 124

III. Results . . . . . . . . . . . . . . 124

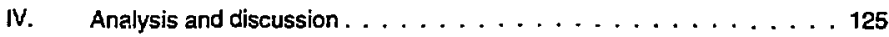

A. Assignment of electronic and vibrational structure . . . . . . 125

B. Inclusion of Jahn-Teller distortion in $\mathrm{Si}_{3}{ }^{\circ} \ldots \ldots \ldots \ldots$

C. Role of the ${ }^{1} A_{1}$ state . . . . . . . . . . . 135

v. Conclusions ................. 136

Chapter 7 . Study of $\mathrm{Si}_{4}$ and $\mathrm{Si}_{4}{ }^{-}$using threshold photodetachment spectroscopy . . . . . . . . . . . . . . . . . 147

I. Introduction $\ldots \ldots \ldots \ldots \ldots \ldots \ldots \ldots \ldots \ldots \ldots$

II. Experimental . . . . . . . . . . . . . 151

III. Results . . . . . . . . . . . . . 152

A. Band A . . . . . . . . . . . . . 153

B. Band $B^{\prime} \ldots \ldots \ldots \ldots \ldots \ldots \ldots . \ldots \ldots$

C. Band $C^{\prime} \ldots \ldots \ldots \ldots \ldots \ldots \ldots \ldots \ldots \ldots$

IV. Analysis and discussion . . . . . . . . . 156

A. Band $\mathrm{A}^{\prime} \ldots \ldots \ldots \ldots \ldots \ldots . \ldots \ldots$

B. Band $B^{\prime} \ldots \ldots \ldots \ldots$. . . . . . . . . . . . . 162 


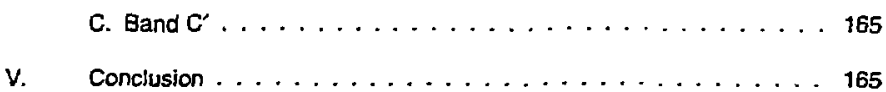

Chapter 8. Study of the low-lying states of $\mathrm{Ge}_{2}-$ and $\mathrm{Ge}_{2}$ using negative ion ZEKE spectroscopy . . . . . . . . . . . . . . . . . . . . . . . 184

I. Introduction .......................... 185

11. Experimental ..................... 188

Ilı. Results . . . . . . . . . . . . . . . . . . 189

IV. Analysis and Discussion .................... 191

A. Triplet band . . . . . . . . . . . . . . . . 192

B. Singlet band . . . . . . . . . . . . . . . . 197

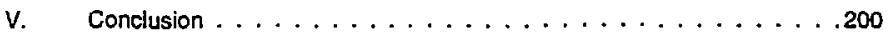

Chapter 9. Study of $\ln { }_{2} P / I n_{2} P$ - and $\ln P_{2} / n P_{2}-$ using negative ion zero electron kinetic energy spectroscopy . . . . . . . . . . . . . . . . . . . . . 212

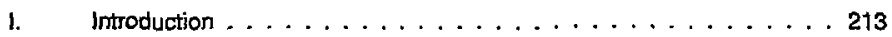

II. Experiment. ...................... 216

III. Results. . . . . . . . . . . . . . . . . 217

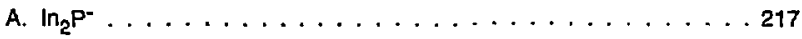

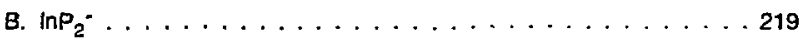

1. Band $x \ldots \ldots \ldots . \ldots . \ldots . \ldots 220$

2. Band A . . . . . . . . . . . . . . 221

3. Ab initio result . . . . . . . . . . . . . 222

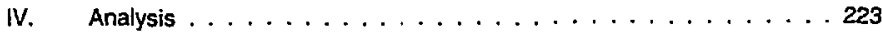

A. $\ln _{2} P-\|_{n_{2}}$ P. . . . . . . . . . . . . . . 224

1. Band $X$ vibrational structure and peak assignments . . . . 224 
2. Approximate geometry differences between the anion and neutral . . . . . . . . . . . . 228

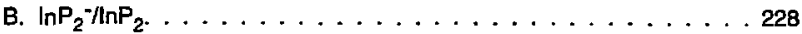

1. Band $X$ vibrational structure and peak assignments . . . . 229

2. Band A vibrational structure and peak assignments . . . . 231

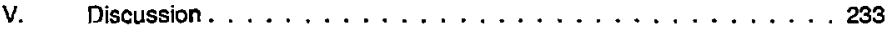

A. $\ln _{2} \mathrm{P}^{-} / \ln _{2} \mathrm{P}$ electronic state assignments . . . . . . . . . 233

B. $\operatorname{lnP}_{2}-\pi n P_{2}$ electronic states .............. 236

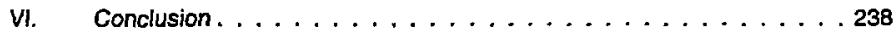

\section{Chapter 10. Study of small gallium arsenide clusters using negative ion}

photodetachment techniques . . . . . . . . . . . . . . . . 254

I. Introduction ..................... 255

H. Experiment ...................... 258

A. Photoelectron spectrometer . . . . . . . . . . . . . . 258

B. ZEKE spectrometer . . . . . . . . . . . . . . . . . 259

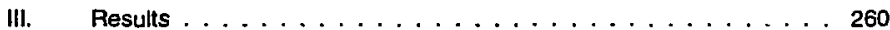

A. PES of $\mathrm{Ga}_{x} A s_{y} \ldots \ldots \ldots \ldots \ldots \ldots$

B. Higher resolution PES of GaAs . . . . . . . . . . . . . . . . 262

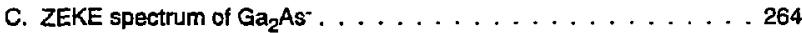

IV. Analysis and Discussion . . . . . . . . . . . 265

A. Electronic structure of $\mathrm{Ga}_{x} \mathrm{As}_{\mathbf{y}} \ldots \ldots \ldots \ldots$

B. Electronic structure of GaAs . . . . . . . . . . . . 268

C. Analysis of the ZEKE spectrum of $\mathrm{Ga}_{2} \mathrm{As}^{*} \ldots \ldots \ldots \ldots . \ldots 271$

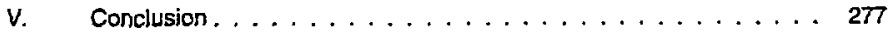


Chapter 11. The power of negative ion ZEKE spectroscopy: an aside on the $1^{-} \cdot \mathrm{CH}_{3} I \mathrm{~S}_{\mathrm{N}^{2}}$ reaction complex. . . . . . . . . . . . . . . . . . . . . 291

I. Introduction . . . . . . . . . . . . . 292

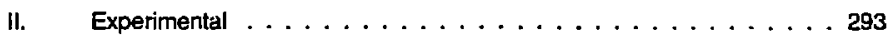

III. Results and Discussion . . . . . . . . . . . . 293

Appendix I. List of publications $\ldots \ldots \ldots \ldots \ldots \ldots \ldots \ldots$ Appendix II. Jahn-Teller interaction code $\ldots \ldots \ldots \ldots \ldots \ldots \ldots$. . . . 30 ? 


\section{Acknowledgments}

Mere words cannot express the admiration and gratitude I feel toward Prof. Daniel $M$. Neunark. His understanding of chemistry and experimeint is inspiring, and 1 feel very fortuna+to have been allowed to join his research group. Over the past five years, I have enjoyed spending his millions for laser dye. gallium arsenide, and materials and labor for ti.e new ZEKE detector. He has allowed my coworkers and me the opportunity to go to conferences both to present data and to have some fun. He gerierously acknowledges his siddents when talking about our restarch, and strc agly supports us in letters of recommendaticn. The ideal research advisor: Amold Schwarzenegyer.

There are several other names which deserve acknowledgment. While every soul one encounters makes same impact on one's life, here are a few who in particu' ar have compelled me toward this end. There is of course my tather, Timothy Chick, who taught me about trees, and my: (late) grandfather, Emost Chick, who challenged my tender mind with questions about aerodynamics and nydrodymamics on our sailing trips.

The darnage that my elementary school did to my sense of capability was repaired by several teachers in junior high school: Mr. Fitzgerald and Mirs. Jones, the very capable science teachers, and Mr. Trombley, who involved me in the math olympics. In high sci nol, I was fortunate enough to have Mi. Kim Kolbe teach me several biology classes. He was a very encouraging and supportive teacher. I would also like to thank Dow Chemical, the place that hired me in high school when all of the fast-food joints rejected imy applications for employment.

When I proceeded to the University of Michigan, Ann Arbor, my interest in biology waned as I was introduced to the subtle allure of chemistry by Prof. Robert Kuzckowski, Prof. Lawrence Bartell, and Prof. Aichard Lawton. Most importantly. Prof. A. H. (Aick) Francis tonk me in and let me build a little experimental apparatus. I really appreciate the time he spent with 
me answering all of my naive questions. Prof. Efrat Lifshitz was also an importanit role model in my undergraduate career.

The experiments described in this thesis would not have been possible had Theo Kitsopoulos not built the original apparatus and trained me to use it. Prof. Irene Waller patiently explained what we were endeavoring to learn with the machine, while Theo taught me how to beat it into submission. I wish the best of luck to the present operators of the ZEKE machine, Yuexing Zhao sid Ivan Yourshaw.

Other members of the group, past and present, have also enhanced my tenure at Berkeley. The useful conversations with Drs. Alex Weaver, Risky Metz, Doug Cyr and, especially, Steve Bradforth, seeing Prof. Bob Continetti actually get a job, getting burritos (at least one per day) and coffee with Dr. Dave Leahy and Dr. Esther deBeer, singing with Dave Ossom, sharing a bed at the ACS with Eun Ha Kim (I couldn't even tell she was there-she didn't even kick me!), excitement over InP clusters with Cangshan Xu, hearing bunny jokes from Dr. Gordon Burton, and telling Eric Ross and Jeft Greenblatt how to be good students have all contributed to my colorful Berkeley experience. The friendship of Cheryn Gliebe (Dan's boss) has also meant a great deal to me. I hope she doesn't mind if I take her to LA with me.

Of course, there is my favorite group member, Don Wesley Amold, whom I liked so much, I married him! In addition to being a loving husband, he has taught me how to run experiments on his photoelectron spectrometer, adapted his fitting code to accommodate my ZEKE data, and he has put up with my computer ignorance like a real hero. He has also managed to put up with my mood swings and weird habits (e.g., overeating, incessant need of diet coke, compulsive shopping and hair chopping, etc.). I aiso appreciate the love and kindness of my in-laws, with wham 1 instantly felt at home.

Heart felt thanks go to Prof. Yuan T. Lee; I was honored that he chaired my preliminary exam committee. I learned a great deal dusing that exam, thanks to him. His kinetics course was invaluable, too. My thanks also go to Prof. Robert Harris, quantum mechanic 
extraordinaire, for also being on my committee along with Prof. Eugene Commins (about whom I ha.J heard a great deal in Prof. Harris' lectures) and Prof. Richard Mathies. 1 also thank Prof. Richard Saykally for his guidance and support, as well as his spotwelder. I know now not to wait too long before having children.

The gentlemen in the machıne shor, especially Eric Graniund, Dave Murai, Bob Dunn, and Hans Graetsch, deserve an enomous amount of thanks for their hard work, and how well they put up with our spastic demeanor and excessive demands.

Finally, I am eternally grateful for the undying, unconditional love and support of my terrific family: Grampa and (greatly missed) Grandma Wickstrom, Grandma Chick and Grandma Penzien, my Aunties (Flash, Judd, Phyllis, Joy) and Uncles, my cousins who have been my dear friends throughout the years, Steve Chick, mon cher frère (whose wave function has had miraculousiy good overlap with mine through our years of wandering) ma belle-sceur, Anne Galy, et leur beau fils, Aurelien, and, most of all, my parents, Maiy and Gene Penzien, without whom I would surely be lost, and the Almighty, without whom there would be nothing to study. 


\section{Chapter 1. Introduction}

A primary objective in the field of atomic and molecular clusters is understanding the evolution of physical and chemical properties of clusters as a function of size from the atomic to bulk scales. In particular, small carbon, silicon, III-V and II-VI clusters composed of fewer than 10 atoms have been the focus of a recent upsurge in both experimental and theoretical work. While these largely uncharacterized species are intriguing by virtue of their novelty, very smail clusters play important roles in several tields of study. For instan, 2 , the small carbon clusters have been found in combustion ${ }^{1}$ and astrophysical studies. ${ }^{2}$ Spectroscopic signal due to interstellar small silicon clusters has also been observed. ${ }^{3}$ The properties of small Group IV, mixed $|1|-V$ and mixed $\mid \mathrm{I}-\mathrm{VI}$ clusters are also of great interest from a technological standpoint because of their potential role in chemicai vapor deposition (CVD) which is a standard method for producing the high grade semiconductor wafers used in research and industry. Moreover, the dynamics and electronics of small clusters may relate to etching processes and defects in the bulk.

Experimental work on small carbon clusters has been ongoing for at least a century, ${ }^{4}$ but the intense interest in the other Group IV and mixed semiconductor clusters, which in part has been fueled by ab initio studies, has been more recent. However, spectroscopic information has proven more difficult to acquire than ab initio results due to the challenges of cluster study. First, most cluster sources simultaneously generate a distribution of cluster sizes, charge, and, 
in some cases, oxides. Second, the slectronic structure of these species are often congested with low-lying electronic states due to their dangling bonds and the potential open shells, making a complete electronic characterization a challenge. While these characteristics of small clusters may hinder more conventional optical spectroscopic techniques, negative ion photodetachment techniques are more easily applied to cluster study for the following reasons. From a practical standpoint, negative ions can be mass-selected prior to spectroscopic investigation, thereby eliminating any ambiguity regarding species identity. Moreover, by detaching an electron from various valence orbitais of the cluster anion, the low-lying neutral states, which often cannot be observed via electronic absorption from the ground neulral state, can be accessed. Vibrational frequencies in totally symmetric modes are generally observed in the ground state as accessed by the anion. The neutral ground state frequencies obtained in this method are therelore complementary to the ground state frequencies obtained in IR absorption studies, which are generally frequencies for non-totally symmetric vibrational modes.

Fixed-Irequency negative ion photoelectron spectroscopy (PES) has been the most common negative ion photodetachment approach to cluster study. Smalley has applied electronicalis resolved negative ion uftraviolet photoelectron spectroscopy (PES) to obtain the electronic band structures of carbon, ${ }^{5}$ silicon, $^{6}$ germanium, $^{6}$ gallium arsenide, ${ }^{7}$ and various metal clusters. ${ }^{8}$ However, the 0.1 to $0.2 \mathrm{eV}$ resolution of Smalley's experiment is such that detailed information about the structures or the electronic states of the ciusters cannot feasibly be extracted from their spectra. Using somewhat higher resolution PES, ranging from 60 to 100 $\mathrm{cm}^{-1}$, negative ion photodetachment techniques have been successfully applied to the investigation of small meta $\left.\right|^{9}$ and semiconductor ${ }^{10}$ clusters, In our own group, we have obtained the PES of $C_{n}{ }^{-}(n=2$ through 11$),{ }^{11} S_{n}^{-}(n=2$ through 8$),{ }^{12}$ and $\ln _{x} P_{y}-(x, y=1 \text { through } 4)^{13}$ with a resolution of 80 to $100 \mathrm{~cm}^{-1}$. The PES of $\mathrm{C}_{n} \cdot \mathrm{Si}_{n}^{-}(n=2$ through 4$)$ and $I n \mathrm{P}_{2}^{-}$were vibrationally resolved, 
However, even the higher resolution PES experiments often cannot resolve the structure due to some of the lower-frequency mades in both the anion and neutrals, which tend to congest the spectra of clusters composed of more than two atoms. On the other hand. negative ion threshold photodetachment (zero electron kinetic energy, or ZFKE) spectroscopy is a technique that offers dramatically better resolution $\left(3 \mathrm{~cm}^{-1}\right)$ than the more conventional PES. While the combination of mass selection and good spectral resolution affordid by the ZEKE technique may appear to render PES redundant, the selection rules are more restrictive for ZEKE than for PES (see below). Because of this, ZEKE spectroscopy is most powerful when used in conjunction with PES. We have obtained ZEKE spectra of $\mathrm{C}_{5},{ }^{-14} \mathrm{C}_{6}{ }^{-15} \mathrm{Si}_{n}^{-}(\mathrm{n}=2,3$ and 4), ${ }^{16,17} \mathrm{Ge}_{2}-\ln _{2} \mathrm{P}^{-}$and $\operatorname{InP}_{2},{ }^{18}$ and $\mathrm{Ga}_{2} \mathrm{As}$. These spectra will be the subjects of the subsequent chapters in this thesis.

As mentioned above, theoretical studies on small clusters have generally preceded experimental investigations. Comparison of the ZEKE spectra with results from ab initio calculations is invaluable in interpreting the cluster spectra. There are several theoreticians in particular who have devoted a tremendous effort toward calculating the properties of these elusive species. Raghavachari's studies on the small silicon cluster studies were in part the motivation for our work on silicon clusters. ${ }^{19}$ His rk showed that, in contrast to the better characterized linear carbon clusters, the silicon clusters tended more toward three-dimensional structures. The striking geometric differences between the calculated ground state structures of carbon and silicon clusters are illustrated in Figure (1.1). Raghavachar has aiso $d \cdot$ ne extensive cakculations on larger silicon clusters, small carbon clusters, ${ }^{20}$ sulfur clusters, ${ }^{21}$ and several mixed semiconductcr clusters. 22

Clusters containing heavier atoms present a more formidable challenge to the theoretician, since the relativistic effects in the heavy atoms can strongly intluence the bonding characteristics in the jiuster. Balasubramanian, however, has carried out geometry calculations, always with an extensive set of electronic states and geometric arrangements on 
species such as $\ln _{3}{ }^{29} \ln _{2} S b$, InSb $b_{2}$, and the gallium arsenide triatomics 24 in addition to several silicon 25 and germanium clusters. 26

From the aforementioned experimental and $a b$ initio results, it has been seen that clusters comiaining only several atoms generally have structural and electronic properties that do not resemble bonding in the bulk. Rather, the structures may have more contracted bonds and compact structures relative to the bulk diamond lattice (or graphite, in the case of carbon) in order to minimize the dangling bonds. This is in contrast to larger nanoparticles containing on the order of several hundred to tens of thousands of atoms, which generally exhibit crystalline structures found in the bulk, but have shifts in the phase diagrams and band structures due to high surface to volume ratios and quantum confinement effects. At which point the small clusters reach a size that the bulk crystal structure becomes eriergetically viable has not yet been experimentally determined, atthough it would appear that mobility measurements should have already determined this. ${ }^{27}$ Ultimately, however, negative ion photodetachment may provide the answer.

\section{Principles of negative ion photodetachment spectroscopy: anion photoelectron spectroscopy and zero electron kinetic energy, or ZEKE spectroscopy}

While the focus of the subsequent chapters is on the application of ZEKE toward cluster study, in practice, these studies usually follow lower-resolution fixed-frequency negative ion photoelectron spectroscopic (PES) work. The results of the ZEKE spectra are then often presented in the context of previously obtained PES. Therefore, a brief description of negative ion PES will precede the description of the principles of ZEKE spectroscopy. Both the PES and ZEKE techniques start with the generation of an intemally cold, mass-selected negative ion beam, but different photodetachment and electron detection schemes are employed. 
PES: In photoelectron spectroscopy, the mass-selected negative ions are photodetached with a fixed-frequency laser yielding a neutral in some vibronic state, and electron with some excess kinetic energy per:

$$
A_{x}\left(v_{n}^{\prime}, T_{0}^{\prime}\right)+e^{-}(K E \neq 0) \stackrel{n, \text { towed }}{\leftarrow} A_{x}^{-}\left(v_{n}^{n}, T_{0}^{\prime \prime}\right) . \quad \text { Eqn. (1.1) }
$$

The kinetic energy distribution of the ejected photoelectrons is measured with an electron timeof-flight analyzer. Figure (1.2) shows a schematic of the energetics involved in this technique. The photoelectron kinetic energies (eKE) are given by

$$
\text { eKE }=h v-E A-T_{0}^{\prime}+T_{v}^{m}-E_{v^{\prime}}^{0}+E_{v^{\prime \prime}}^{\prime} \text {, }
$$

where $\mathrm{hv}$ is the detachment laser photon energy, EA is the neutral electron affinity, $\mathrm{T}_{e}^{\prime}$ and $\mathrm{T}_{e}^{\prime \prime}$ are the term values of the specific neutral and anion electronic states, respectively, and $E_{v^{\prime}}^{0}$ and $E_{v}$ are the vibrational energies with respect to the zero point energies of the neutral and anion states, respectively. The electron kinetic energy distribution is therefore peaked from transitions between anion and neutral levels, as shown in Figure (1.2).

Another analytical tool in PES is the use of laser polarization to discem between overlapping electronic transitions which often occur in clusters. Photoelectrons detached from different mclecular orbilals of the snion (which accesses the different neutral electronic states) will generally have different angular distributions with respect to the laser polarization The intensity of a particular electronic band in the spectrum will therefore change relative to other electronic bands at altemative polarizations.

The intensity of transitions from a particular vibronic level of the anion to different vibrational levels within an electronic state of the neutral, $v^{\prime \prime}$ and $v^{\prime}$, respectively, is assumed proportional to the Franck-Condon factors, 


$$
\text { Intensity } \propto\left|\left\langle v^{\prime \prime} \mid v^{\prime}\right\rangle\right|^{2}
$$

If the anion and neutral are of the same symmetry, transitions between the . nd vibronic state of the anion to totally symmetric vibronic levels in the neutral will be the most intense in the spectrum. Moreover, geometry differences between the anion and neutral can be estimated by simulating the observed vibrational progressions. This can be achieved most simply by approximating the anion and neutral vibrational wave functions as products of hammic oscillator wave functions. The displacement of the anion wave function along the appropriate neutral coordinate can then be varied until the simulated progression profiles match the spectrum per Eqn. (1.3). In some cases, the displacements obtained in this manner can be transformed into actual bond distance and angle differences between the anion and neutral geometries.

The energy resolution of the electron time-ot-flight analyzer in the spectrometer used in the studies presented in this work is $8 \mathrm{meV}$ at $0.65 \mathrm{eV}$ eKE and degrades as (eKE) ${ }^{3 / 2}$ at higher electron kinetic energies. While this should be adequate to resolve most symmetric stretch progressions in the neutral carbon and silicon clusters, this is insufticient to discem rotational structure and lower frequency modes that may be active, in addition to the hot bands and sequence bands that may congest the spectra.

ZEKE: Considerably higher resolution spectra can be obtained using negative ion ZEKE spectroscopy. The energetics involved in this technique are illustrated in Figure (1.3). The method of ZEKE spectroscopy follows

$$
A_{x}\left(v_{n}^{\prime}, T_{0}^{\prime}\right)+e^{-}(K E \approx 0) \stackrel{\text { m,seanned }}{\leftarrow} A_{x}^{-}\left(v_{n}^{\prime \prime}, T_{0}^{\prime \prime}\right) .
$$


Mass-selected negative ions are photocetached with a tunable pulsed laser rather than a fixedfrequency laser, and only those electrons with nearly zero kinetic energy are detected. This is in contrast to a total photodetachment cross section spectrum, which is obtained by collecting all of the photoelectrons as a function of photon energy while tuning the detachment laser through the anion detachment continuum. The total cross section would ideally resemble that shown on the upper right side of Figure (1.3): An onset of electrons occurs at detachment threshold, and at the threshold of accessing a higher-lying energy level of the neutral, more eiactron signal would be collected.

Because the ZEKE spectrum is obtained by discriminating against energetic electrons and collecting only those electrons detached with no excess kinetic energy (i.e., those detached at the threshold of a neutral $\leftarrow$ anion transition) the electron signal plotted against the detachment waveiength consists of peaks, as is illustrated in the lower right side of Figure (1.3). Each peak corresponds to transitions between energy levels of the anion and the neutral, since nearly zero kinetic energy electrons (ZEKE's) will be produced only when the photon energy is equal to such a transition.

Discrimination of energetic electrons is achieved by a delayed collection scheme. The negative ions are detached in a field free region, and the energetic electrons are allowed to scatter away from the neutrals and undetached anions, while the ZEKE electrons, with little to no kinetic energy relative to the neutrals and undetached lons will therefore remain close. After a certain period of time has passed (typically on the order of 200 to $600 \mathrm{~ns}$ ), a gentle field extracts the remaining ZEKE electrons to an electron detector. In principle, the longer the ZEKE extraction is delayed, the more efficiently the energetic electrons will be discriminated against, and the better the resolution. A more detailed description the electron detection scheme can be found in Chapter 2.

This method of selectively detecting low kinetic energy eiectrons is similar to techniques developed by Müller-Dethlefs et al.28 for threshold photoionization of neutrals. However, there is 
a fundamental difference between ZEKE of neutral species and ZEKE of anions. Namely, in ZEKE of neutrals, neutrals are typically excited to an extremely high-lying neutral Rydberg st:te lying just below the detachment continuum, or a quasibound vibrationally excited fydberg state. After allowing some time for any energetic electrons to scatter, the bound or quasi-bound neutral is field ionized by some pulsed extraction field. Because the electron is bound prior to field ionization, very long (on the order of microseconds) delay times can be employed. The quality cf the neutral ZEKE spectrum is therefore sensitive io the ionizing field strength and the shape of the pulse. In contrast, ZEKE of anions involves completely free low-kinetic energy electrons which are notoriously susceptible to stray electric or magnefic fieids, including the repulsive fields (space charge effects) from nearby undetached anions. The space charge effects are typically what limits the resolution of negative ion ZEKE to 2 to $3 \mathrm{~cm}^{-1}$.

Because the electrons detected in an anion ZEKE are free and have very low-kinetic energy, the anion ZEKE experiment is inherently more difficult than a neutral ZEKE experiment. However, in anion ZEKE, the benefit is that one obtains information regarding neutral state:s, while in neutral ZEKE, the positive ions, or high-lying neutral states that resemble the positive ions, are accessed. Also, the peak intensities in anion ZEKE spectra are easier to interpret than in neutral ZEKE, as they are not confounded by Rydberg state lifetimes or coupling with other close-iying Fydberg states.

\section{The Wigner threshold law, and how it applies to polyatomics}

While it is desirable to obtain the higher resolution threshold photodetachment spectra for all of the carbon clusters studied using photoelectron spectrcscopy, the abiitity to acquire threshold photodetachment spectra is contingent on the photodetachment cross section near threshold. The Wigner threshold law ${ }^{29}$ gives that near threshold, the photodetachment cress section for an atomic system goes as 


$$
\sigma \propto \sigma_{0}\left(E_{h v}-E_{\text {threshold }}\right)^{f+1 / 2}
$$

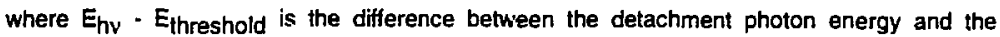
transition energy between an anion and neutral level, and $\ell$ is the angular momentum imparted upon the photoelectron. For $\ell=0$ (s-wave detachment) the cross section rises shaply above threshold, but for $\ell \geq 1$ ( $p, d, \ldots$-wave detachment), the cross section is very small near thresinold, making the ZEKE technique insensitive to transitions other than those involving swave detachment.

Reed ef al..$^{30}$ extended the threshold law to polyatomic anions with the end result that orbitals that transform as $x, y$, or $z$ in the anion symmetry can yield s-wave phctoelectrons (as well as higher wave electrons). For the linear homonuclear species we have studied, in which the $\pi_{\mathrm{u}}$ and $\sigma_{\mathrm{u}}$ orbitals transform as $(x, y)$ and $z$, respectively, we have found this rule to satisfactorily predict the threshold detachment behavior. As expected, of the small carbon clusters, we were able to obtain ZEKE spectra of $\mathrm{C}_{5}{ }^{-}$and $\mathrm{C}_{6}{ }^{+}$, as the neutral $\leftarrow$ anion transition involves the removal of a $\pi_{u}$ electron (we have not yet been successful in our attempts to obtain the ZEKE spectrum of $\mathrm{C}_{9}$ ", which should also detach via s-wave, possibly due to strong anion $\leftarrow$ anion transitions complicating the total photodetachment cross section- see Chapter 4).

In many cases, particularly in nonlinear polyatomics, no ZEKE signal is observed. This may be due to the lack of a molecular axis upon which orbital angular momentum projection is meaningful. The ejected photoelectrons generally have contributions from many waves, and how closely the molecular orbital resembles some $p$ - or $\pi_{u}$-type orbital determines the magnitude of the s-wave component in the departing photoelectron. For instance in a triatomic $\mathrm{C}_{2 \mathrm{v}}$ molecule, of the four characters $\left(a_{1}, a_{2}, b_{1}\right.$ and $\left.b_{2}\right)$ only $a_{2}$ does not transform as $x, y$, or $z$. However, we have found empirically from our studies on $\mathrm{Si}_{3}{ }^{-}$and $\ln _{2} \mathrm{P}^{-}$that the photodetachment cross section for an electron in an $a_{1}$ orbital mav be too small to observe any 
ZEKE signal. $A_{1}$ obitals in $C_{2 v}$ molecules have no plane through which reflection results in a change in the sign of the wave function, as is the case with a p-orbital, and in the cases mentioned above, this may be the reason for the small cross section near threshold. However, consider an $a_{1}$ orbital composed of primarily the $p_{2}$ orbitai $c n$ the apical atom, or even composed of the two $p_{z}$-orbitals on the two side atoms. Such $a_{1}$ orbitals may yield photoelectrons with strong s-wave contribution. This may be the case for a molecule such as $\operatorname{lnP}_{2}$, in which the bonding is reminiscent of a partially positive iri atom loosely bound to a partially negative $P_{2}$ diatom, and the $a_{1}$ orbital can be described as a $\pi_{4}$ bond between the two $P$ atorns, but in the plane of the $\operatorname{lnP}_{2}$ molecule.

On the other hand, virtually no photodetached electrons were observed near detachment threshold for the bipyramidal $\mathrm{Si}_{5}{ }^{-}\left(\mathrm{D}_{3 h}\right), \mathrm{Si}_{6}{ }^{-}\left(\mathrm{D}_{4 h}\right)$, and $\mathrm{Si}_{7}{ }^{-}\left(\mathrm{D}_{5 \mathrm{~h}}\right)$ clusters $\left[\mathrm{Si}_{5}\right.$ and $\mathrm{Si}_{6}$ are shown in Figure (1.1)l even though ab initio calculations predict the electron to be detached from a $p_{2}$-like orbital $\left(a_{2}^{\prime \prime}, a_{2 u}\right.$, and $a_{2}{ }^{\prime \prime}$, respectively). However, calculations determining the shapes of the orbitals may show that even though reflection through the bipyramidal plane changes the sign of the orbital wave function, the distribution of " + " and "-" density on either side of the plane may cancel out any overlap with a p-orbital. In these species, there is also a substantial geometry difference between the anion and neutral, which may contribute to the problem of low ZEKE signal.

\section{Application of negative ion photodetachment techniques toward the charasterization of small carbon and semiconductor clusters}

Some of the interesting issues in small cluster study revolve around how cluster geometries and electronics evolve as a function of the number of atoms, and how clusters composed of materials in the same periodic group compare to one another. For instance, 
Figure (1.1) shows the dramatic structural differences between carbon clusters and silicon clusters. ${ }^{19,20}$ The linear cumulenic structures of the small carbon clusters reflect how $\pi$-bonding is favored in carbon atoms, while the tei ijency for silicon to form bent or three dimensional structures reflects how $\pi$-bonding is acommon for silicon. Cyclic structures, in which cumulenic bonding dominates, are afso predicted to be nearly degenerate with the linear structures for the even numbered carbon clusters with four or more atoms, ${ }^{20}$ although very little experimental evidence for the existence of the cyclic structures presently exists. This may be due to the high temperature in the initial stages of cluster fomation in laser vaporization sources.

The structural differences in these species can be inferred from their negative ion photodetachment spectra. Figure (1.4) shows the negative ion PES of several small carbon"1 and silicon clusters obtained using a $4.65 \mathrm{eV}$ detachment energy. The contrasts between the $\mathrm{C}_{n}^{-}$and the $\mathrm{Si}_{n}^{-}$spectra are striking; the $\mathrm{C}_{n}^{-}$spectra are dominated by sharp and intense origin transitions while the $\mathrm{Si}_{n}{ }^{-}$spectra, particulariy for $\mathrm{n}>4$, ire congested by oroad and vibrationally unresolved electronic bands. The carbon cluster spectra show the characteristic ndd-even altemation of electron affinities with the even numbered clusters having higher electron affinities than the odd numbered clusters. This is consistent with their linear, cumule.ic structures, in which the odd numbered clusters closed-sheil electron configurations, as opposed to the openshell configurations of the even numbered clusters. The electron affinities also increase as a function of cluster size within the even or odd series.

With the $\mathrm{Si}_{n}-\mathrm{PES}$, size trends are not as obvious, except that the spectra get more congested as a function of size. The especially broad ongin transitions for the larger clusters indicate trat the LUMO of the neutral species has a profound effect on the cluster geometry. $A b$ intitio calculitions predict the LUMO's for $n=5,6$, and 7 are antibonding between the apical atoms of the bipyramids. However, with so many close-lying neutral electronic states, vibronic coupling may also contribute to the congestion. 
The differences between small silicon and germanium clusters are more subtie. Lower resclution UV.PES investigations on $\mathrm{Si}_{n}{ }^{-}$and $\mathrm{Ge}_{n}{ }^{-}$by Smalley et al. ${ }^{6}$ revealed virtually identical electronic structure for the two elements. The ZEKE spectra of $\mathrm{Si}_{4}{ }^{-}$and $\mathrm{Ge}_{4}{ }^{-}$shown in Figure (1.5) exhibit a resolved vibrational progressions in the $v_{2}$ mode of the ${ }^{3} B_{3 u}$ first excited neutral state over the same wavelength range (the transition to the ground neutral state carn not be observed ir the ZEKE spectrum, as it involves p-wave detachment, see Sec. II). This range corresponds to the eKE $=1.4$ to $1.7 \mathrm{eV}$ range on the PES of $\mathrm{Si}_{4}{ }^{-}$shown in Figure $(1,4)$. Aside from the difference in quality of the two spectra, the $\mathrm{Ge}_{4}{ }^{-}$progression has a $174 \mathrm{~cm}^{-1}$ spacing while the $\mathrm{Si}_{4}^{-}$progression has a $311 \mathrm{~cm}^{-1}$ spacing. Considering that $\omega_{e}=\left(k^{\prime} \mu\right)^{1 / 2}$, this means that $\mathrm{K}_{2, \mathrm{Si} 4}=1.23 \mathrm{k}_{2, \mathrm{Ge4}}$. That the $\mathrm{Ge}_{4}$ force constant is smaller is consistent with bonding trends in the penodic table.

These are just a few brief examples of how negative ien photodetachment technirives can be applied to study cluster properties. Chapter 2 describes several improvements to the ZEKE apparatus implemented to obtain the spectra. Chapters 3 through 10 give more detailed descriptions and analysis of ZEKE spectra of carton, silicon, germanium, indiurh phosphide, and gallium aresenide clusters. Finally, chapter 11 presents and discusses the ZEKE spectrum of the $1 \cdot \cdot \mathrm{CH}_{3} l \mathrm{~S}_{\mathrm{N}} 2$ reaction complex, showing that ZEKE spectroscopy is a powerful tcol for studying dramatically different types of systems. 
1P. Gehardt, S. Loffler, and K. H. Homann, Chem. Phys. Lett. 137, 306 (1987); J. B. Edwards, Combustion, Fomation, and Emission of Trace Species (Ann Artor Science, Ann Arbor, 1974); J. Heath, S. C. O'Brien, R. F. Cur, H. W. Kroto, and R. E. Smalley, Comments Condensed Matter Phys. 13, 119 (1987); H. W. Kroto and K. McKay, Nature 331, 328 (1988).

2A. E. Douglas, Nature 269, 130 (1979); R. F. Knake, ibjd. 269, 132 (1979); H. W. Kroto, J. R. Heath, S. C. O'Brien, R. F. Curt and R. E. Smaliey, Astrophys. J. 314, 352 (1987); K. H. Hinkle, J. J. Keady, and P. F. Bemath, Science 241, 1319 (1988); P. W. Merill, Publ. Astron. Soc. Pac. 38, 175 (1926); R. F. Sanford, Astrophys. J. 111, 262 (1950); B. Kleman. Astrophys. J. 123, 162 (1950).

3S. P. Davis and J. W. Brault, J. Opt. Soc. Am. B 4. 20 (1987).

${ }^{4}$ For instance, some of the work on $\mathrm{C}_{3}$ includes W. Huggins, Proc. R. Soc. London 33,1 (1882); G. Herzberg, Astrophys. J. 96, 314 (1949); L. Gausset, G. Herzberg, A. Lagerquist, and B. Rosen, Discuss. Faraday Soc. 35, 113 (1965); C. A. Schmuttenmaier, R. C. Cohen, N. Pugliano, J. R. Heath, and R. J. Saykaliy, Science 249, 897 (1990).

5L. S. Wang, J. Conceicao, C. M. Jin, and R. E. Smalley. Chem. Phys. Lett 182, 5 (1991); S. Yang, K. J. Taylor, M. J. Craycraft, J. Conceicao, C. L. Pettiette, O. Cheshnovsky, and R. E. Smalley, ibid. 144, 431 (1988).

${ }^{6}$ O. Cheshnovsky, S. H. Yang, C. L. Pettiette, M. J. Craycratt, Y. Liu, and R. E. Smalley, Chem. Phys. Lett. 138, 119 (1987).

7C. Jin, K. J. Taylor, J. Conceicao, and R. E. Smalley, Chem. Phys. Lett. 175. i7 (1990).

BK. J. Taylor, C. L. Pettiette, O. Cheshnovsky, and A. E. Smalley, J. Chem. Phys. 96,3319 (1992).

${ }^{9}$ For example, see J. Ho, K. M. Ervin, and W. C. Lineberger, J. Chem. Phys. 93, 6987 (1990);J. Ho, M. L. Pollak, K. M. Ervin, and W. C. Lineberger, J. Chem. Phys. 99.8542 (1993); J. G. 
Eaton, H. W. Sarkas, S. T. Arnold, K. M. MeHugh, an K. H. Bowen, Chem. Phys. Lett. 193, 141 (1992); G. Gantefor, M. Gausa, K. H. Meiwesbroer, H. O. Lutz, Faraday Disc. Chem. Soc. 86. 197 (198); Z. Phys. D 9, 253 (1988); S. M. Casey, P. W. Villalta, A. A. Bengali, G. L. Cheny, and D. G. Leopold, J. Am. Chem. Soc. 113, 6688 (1991).

10M. R. Nimlos, L. B. Harding, and G. B. Ellison, J. Chem. Phys. 87, 5116 (1987).

${ }^{11}$ D. W. Amold, S. E. Bradforth, T. N. Kitsopoulos, and D. M. Neumark, J. Chem. Phys. 95, 8753 (1991).

${ }^{12}$ T. N. Kitsopoulos, C. J. Chick, Y. Zhao, and D. M. Neumark, J. Chem. Phys. 95, 1441 ;1991); C. C. Amold, T. N. Kitsopoulos, and Daniel M. Neumark, J. Chem. Phys. 99, 766 (1993); T. N. Kitsopoulos, C. J. Chick, A. Weaver, D. M. Neumark, J. Chem. Phys. 93, 6108 (1990).

${ }^{13}$ C. Xu, E. de Beer, D. W. Amold, C. C. Amold, and D. M. Neumark, J. Chem. Phys. 101 (1994).

${ }^{14}$ T. N. Kitsopoulos, C. J. Chick, Y. Zhao, and D. M. Neumark, J. Chem. Phys. 95, 5479 (1991).

${ }^{15}$ C. C. Amold, Y. Zhao, T. N. Kitsopoulos, and D. M. Neumark, J. Chem. Phys. 97, 6121 (1992).

16C. C. Amold and D. M. Neumark, J. Chem. Phys. 100, 1797 (1994).

${ }^{17}$ C. C. Amold and D. M. Neumark, J. Chem. Phys. 99, 3353 (1993).

${ }^{18}$ C. C. Amold and D. M. Neumark, accepted for publication, Can. J. Phys. (1994).

19K. Raghavachari and V. Logovinsky, Phys. Rev. Lett. 55, 2853 (1985); K. Raghavachari, J. Chem. Phys. 84, 5672 (1986); K. Raghavachari and C. M. Rohlfing, Chem. Phys. Lett. 143, 428 (1988); J. Chern. Phys. 89, 2219 (1988).

20K. Raghavachari and J. S. Binkley, J. Chem. Phys. 87, 2191 (1987). 
21K. Raghavachari, C. M. Rohifing, and J. S. Binkley, J. Chem. Phys. 93, 5862 (1990).

22M. A. Al-Lam, G. W. Trucks, and K. Raghavachari, J. Chem. Phys. 96, 1137 (1992);M A. AlLaham and K. Raghavachan, Chem. Phys. Lett. 187, 13 (1991).

23P. Y. Feng and K. Balasubramanian, Chem. Phys. 138, 89 (1989).

${ }^{24}$ K. K. Das and K. Balasubramanian, J. Chem. Phys. 94, 6620 (1991).

${ }^{25}$ K. Balasubramanian, Chem. Phys. Lett. 125, 400 (1986); Chem. Phys. Lett. 135, 228 (1987)

26K. Balasubramanian, J. Mol. Spectrosc. 123, 228 (1987).

27 There appears to be some size overlap in the silicon clusters studied by M. F. Jarrold and J. E. Bower using mobility measurements [J. Chem. Phys. 96, 9180 (1992)] and P. E. Batson and J. R. Heath [Phys. Rev. Lett. 71, 911 (1993)] who used electron microscopy and EELS to determine some crystallinity in smaller silicon nanocrystals.

${ }^{28}$ K. Müller-Dethlefs, M. Sander, and E. W. Schlag, Z. Naturtorsch, 39a, 1089 (1984); Chem. Phys. Lett. 12, 291 (1984).

${ }^{29}$ E. P. Wigner, Phys. Rev. 73, 1002 (1948).

30K. J. Reed, A. H. Zimmerman, H. C. Anderson, and J. I. Brauman, J. Chem. Phys. 64, 1368 (1976). 
Figure (1.1) Comparison of the structures of small carbon and silicon clusters (the low-lying cyclic structures predicted for $\mathrm{C}_{4}$ and $\mathrm{C}_{6}$ are nol shown).
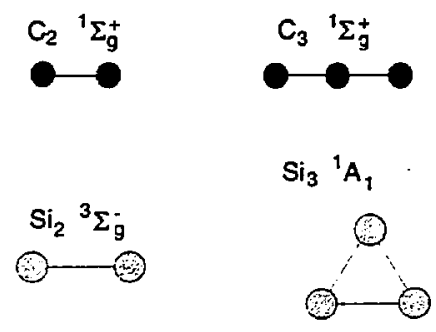

$\mathrm{Si}_{3}{ }^{1} \mathrm{~A}_{1}$
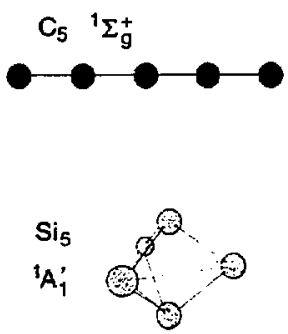
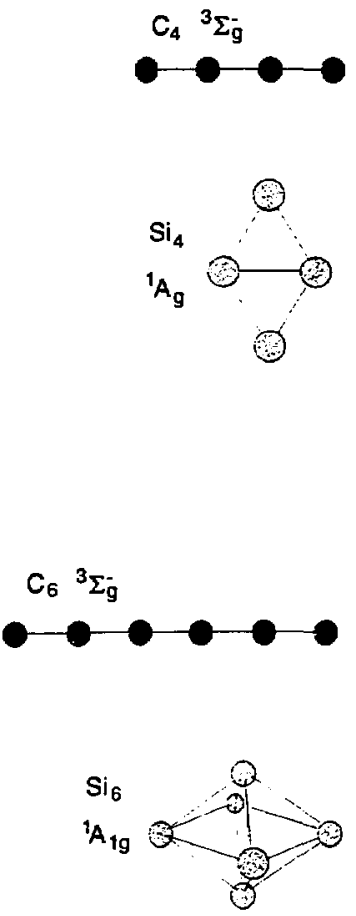
Figure (1.2) Energetic considerations in negat've ion fixed-frequency photoelectron spectroscopy.

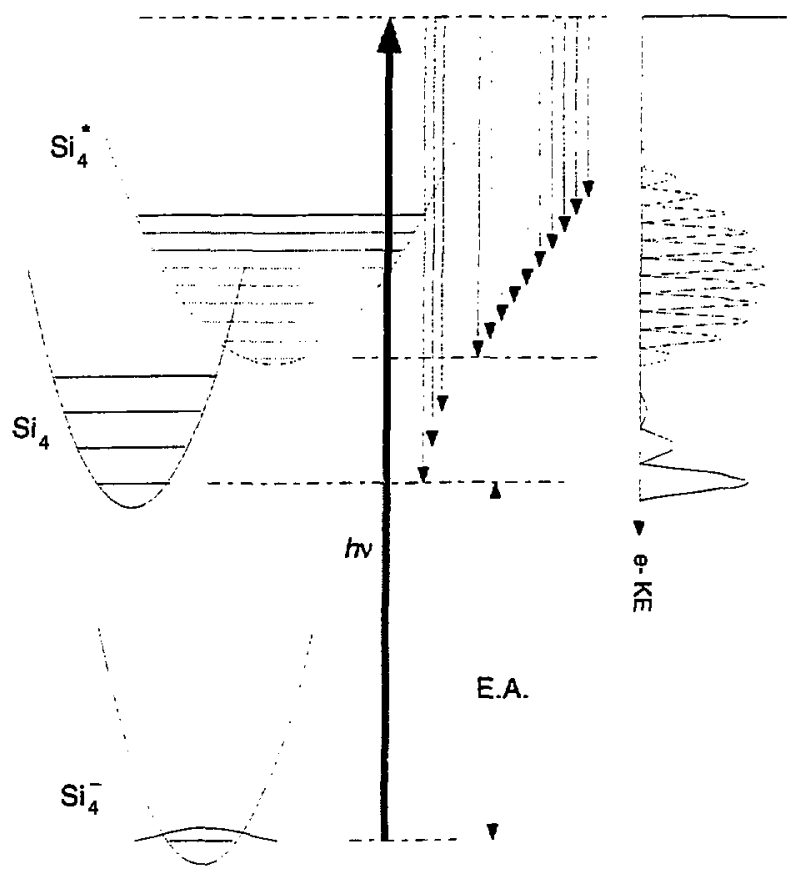


Figure (1.3) Energetic considerations in negative ion ZEKE spectroscopy.

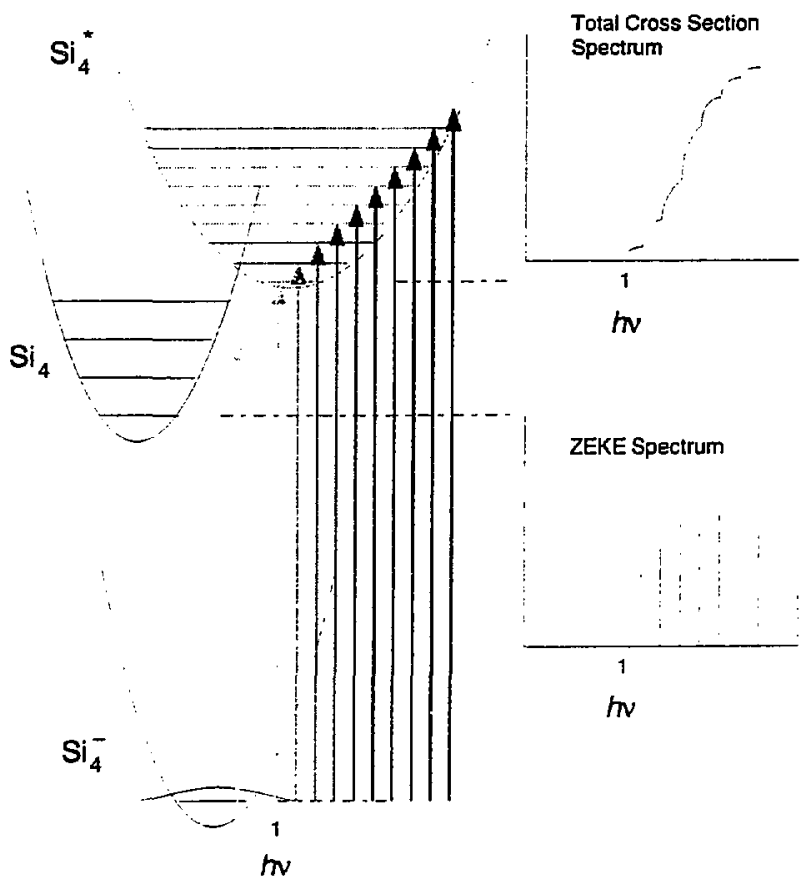


Figure (1.4) PES of several small carbon and silicon clusters obtained using $4.66 \mathrm{eV}$ photon energy.

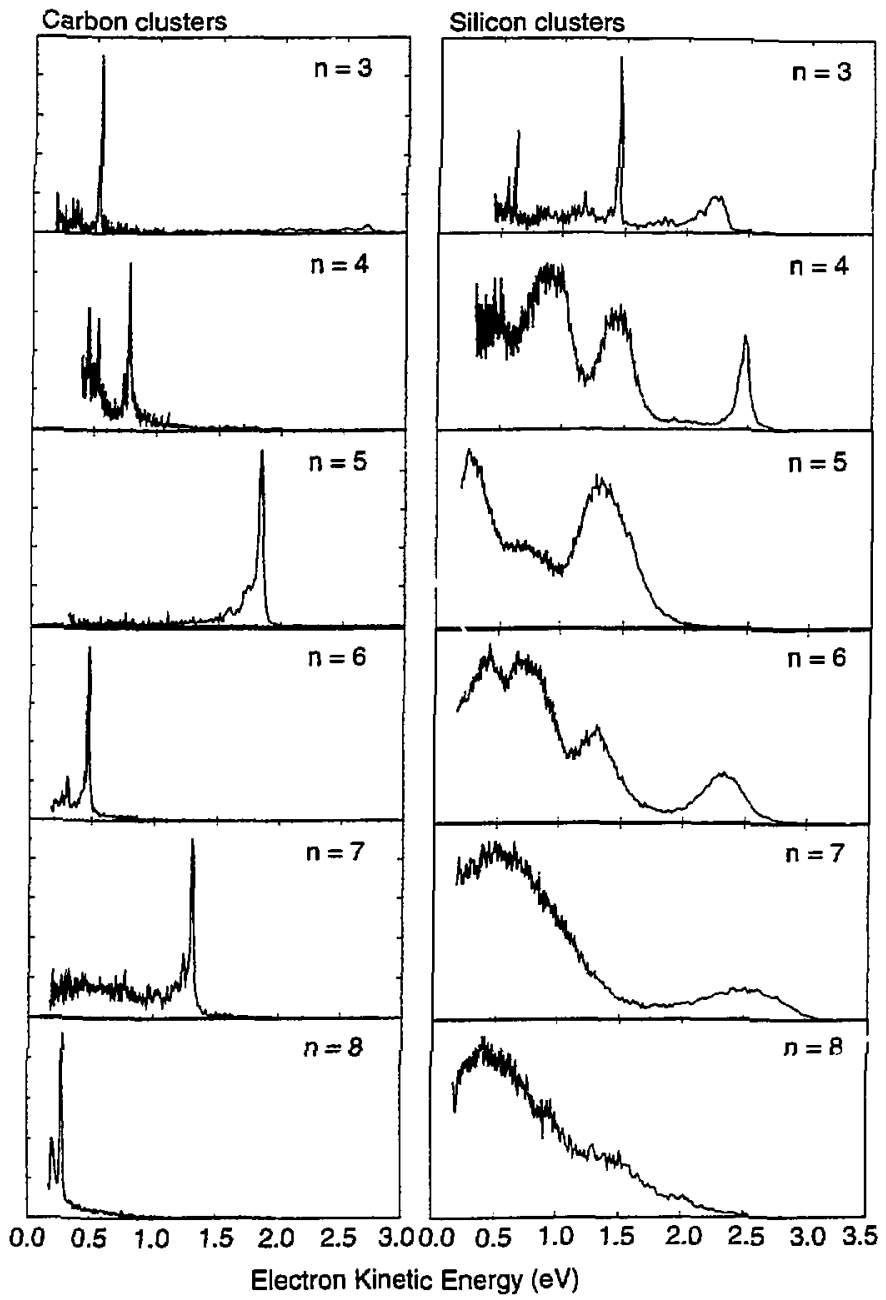


Figure (1.5) ZEKE spectra of $\mathrm{Si}_{4}{ }^{*}$ and $\mathrm{Ge}_{4} ;{ }^{*} \mathrm{~B}_{3 u}+e^{-} \leftarrow{ }^{2} \mathrm{~B}_{2 g}$ transition.

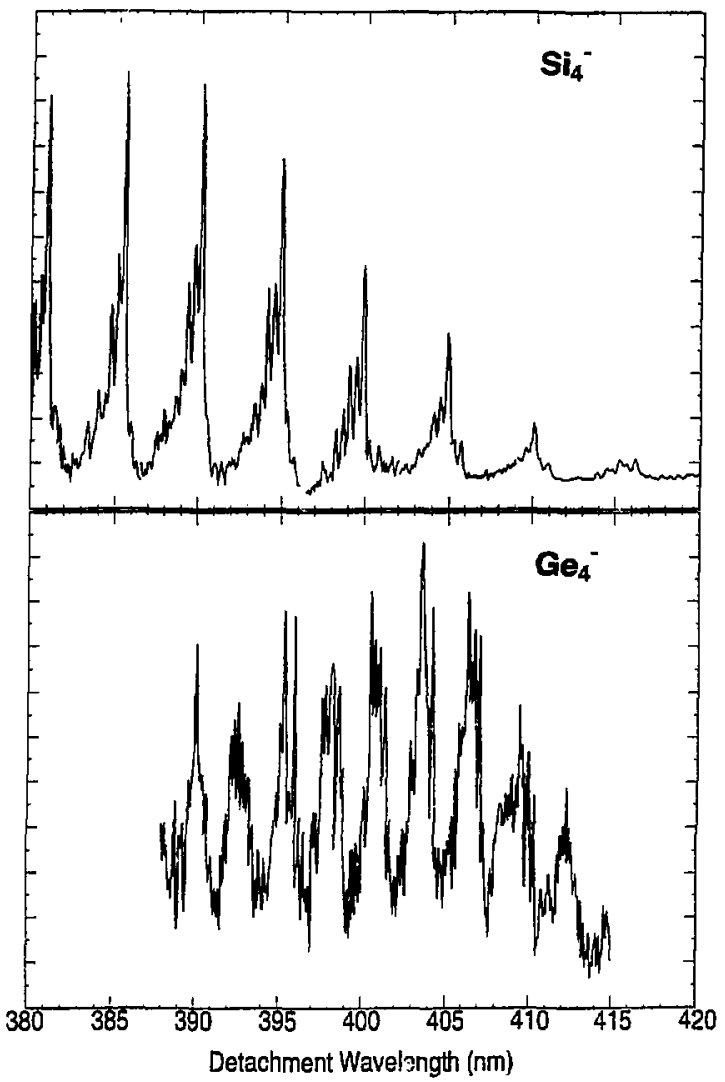




\section{Chapter 2. Experimental modifications to the threshold photodetachment spectrometer}

"If it were easy, everyone would be doing it."

- Daniel M. Neumark

The ZEKE apparatus as it was buitt by Kitsopoulos et al. has been described in detail elsewhere, ${ }^{1}$ and aside from the several modifications described in this chapter, the basic operation is the same. A schematic of the threshold photodetachment spectrometer is shown in Figure (2.1). Briefly, cluster ions are generated in a ion source chaniber, and then enter a massspectrometer. After mass-separation, the ion of interest is detached by a tunable light source, and only those electrons released within several $\mathrm{cm}^{-1}$ of a neutral $\leftarrow$ anion transition are selectively detected for the ZEKE spectrum.

The ions are generated in a cluster source based on the design developed by Smalley 2 (1) which will be discussed in Greater detail below. The negative ions that pass trough a $2 \mathrm{~mm}$ skimmer are colinearly accelerated to $1 \mathrm{keV}$. Mass-selection is achieved with a $1 \mathrm{~m}$ long beammodulated time-of-flight mass spectrometer. ${ }^{3}$ 'on optics including an Einzel lens and a temporal compressor are situated in the ion flight tube. The mass-separated ions then pass through a 3 
$\mathrm{mm}$ mass spectrometer defining aperture into the detection region where they are photodetached in the region labeled (2) by a (pulsed) excimer-numped dye laser $60 \mathrm{~cm}$ upstream of the ion (3) and electron (4) detectors (the electron detector is located out of the plane above the \&).

The selective detection of the near-threshold electrons having nearly zero kinetic energy in the ion beam frame (ZEKE's) is achieved by photodetaching the anions wher the interaction region (2) is initially field-free. A weak extraction pulse $(1-4 \mathrm{~V} / \mathrm{cm})$ is then applied along the ion beam axis 200 to $300 \mathrm{~ns}$ after photodetachment occurs. The delay betwetun detachment and extraction allows the higher kinetic energy electrons to spatially separate from the nearly zero kinetic energy electrons. Energetic electrons that scatter perpendicular to the ion beam axis are blocked upon extraction by the apertures (5) located between the detachment region and the electron detector, while those that scatter along the ion beam axis will emerge from the extraction region with different kinetic energies than the threshold electrons, giving them ditferent arrival times at the detector. Discrimination against the energetic electrons is then achieved by gated integration of the threshold electron signal.

This combination of spatial and temporal fiftering of energetic electrons is based on techniques developed by Müller-Dethlefs ef al. ${ }^{4}$ for the photodetachment (through pulsed-field ionization) of neutrals, and gives an energy resolution of as good as $3 \mathrm{~cm}^{-1}$. For several of the spectra presented in subsequent chapters, particularly the $\mathrm{Si}_{2}^{-}$(Chapter 5 ) and $\mathrm{Si}_{3}{ }^{-}$(Chapter 6 ) spectra, resolution was sacrificed to increase the electrorı signal. The resolution of the apparatus as it was employed for these two species is estimated to be -10 to $15 \mathrm{~cm}^{-1}$.

\section{The cluster source}

As mentioned above, a cluster source similar to that developed by Smalley and coworkers $^{2}$ has been employed in all of the cluster studies described in the subsequent 
chapters. A schematic of the cluster source is shown in Figure (2.2), and it is comprised of three major components: a molecular beam valve, a "source" piece, and a clustering channel. Briefly, the surface of a 0.25 inch OD (carbon, silicon, germanium, InP. GaAs) rod is ablated with the focused and attenuated 2nd hamonic (532 nm) output of a Nd:YAG laser operated at $20 \mathrm{~Hz}$ repetition rate. The rod is kept translating and rotating, so that a fresh surface is ablated with each laser shot. The resulting plasma is entrained in a pulse of a rare gas from a molecular beam valve, and then expanded through the clustering channel where clusters and cluster ions can aggregate and cool via collisions with other clusters or rare gas atoms. Helium is generally used as the carrier gas. It was easier to generated vibrationally cold ions using neon as the carrier gas, ${ }^{5}$ but neon and other heavier carrier gases also made it necessary to use greater vaporization power, which, as described below, has an undesired effect on the quality of the ions. It was also found that similarly cold ions could be generated using He by manipulating the timing between firing the molecular beam valve and the vaporization laser. The intersection of the rod, the laser beam, and the molecular beam occurs in the aluminum source piece. which attaches to both the face plate of the molecular beam valve and the clustering channel. After exiting the clustering channel, those clusters having negativa charge and that pass through a 2 $\mathrm{mm}$ skimmer are accelerated to $1 \mathrm{keV}$ into a flight tube, where the mass separation and subsequent spectroscopic investigation occurs.

The cluster source is flexible in that there are numerous parameters that can be adjusted to affect the cluster mass distribution and vibrational temperature. For instance, laser vaporization power, molecular beam valve type, carrier gas, carrier gas backing pressure, carrier gas pulse duration, timing between ablation and entrainment. length and inner diameter uf the modular clustering channel, and having or not having an exit slit or cone at the end of the clustering channel are all important parameters. The $\mathrm{Si}_{2}-$ and the $\mathrm{C}_{5}-\mathrm{ZEKE}$ spectra were farty insensitive to cluster source conditions. Changing the backing pressure of the carrier gas, or grossly changing the timing af the laser beam pulse with respect to the molecular beam pulse 
was found to affect the vibrational temperature of these species but as long as these two ions could be made, their spectra could be obtained. In general, however, the vibrationally coldest cluster ions were obtained using a $0.2^{n}$ long, $0.15^{n}$ ID clustering channel with a $0.1^{\prime \prime}$ ID exit slit. This is the configuration shown in Figure (2.2). The timing between the triggering of the beam valve and the ablation laser should be such that the laser hits the rod on the early part of the rising edge of the carrier gas pulse. The General Valve driver used in these studies takes slightly longer than the 250 us delay between the YAG trigger and actual output, so the YAG was triggered 40 to $60 \mu$ s after the General Valve trigger.

The ability to obtain spectra of reasonable quality for the larger clusters, however, has tumed out to be critically sensitive to a fairly narrow range of source conditions for each material. The $\mathrm{C}_{6} \cdot \mathrm{Si}_{3}$ and $\mathrm{Si}_{4}^{-}$were much more difficult to obtain than the $\mathrm{C}_{5}^{-}$and $\mathrm{Si}_{2}{ }^{-}$because of the need for much colder source conditions. No distinct threshold was observed in the total photodetachment cross section spectrum of $\mathrm{C}_{6}-$ until the vaporization power was tumed down to $4 \mathrm{~mJ} / \mathrm{pulse}$, compared to the $8 \mathrm{~mJ} /$ pulse vaporization power typically used for $\mathrm{Si}_{2}{ }^{-}$and $\mathrm{C}_{5} \cdot \mathrm{A}$ change in the beam valve had to be made in order to obtain $\mathrm{Si}_{3}{ }^{-}$and $\mathrm{Si}_{4}{ }^{-} \mathrm{ZEKE}$ spectra.

\section{A. Piezoelectric molecular beam valve}

When $\mathrm{Si}_{4}{ }^{-}$was generated using the same source conditions we used to obtain good $\mathrm{C}_{6}{ }^{-}$ spectra, not ZEKE structure was observed. When the vaporization power was dropped to 2 $\mathrm{mJ} /$ pulse, some structure was observed, but it was broad and congested. However, when a piezoelectric beam valve similar to that designed by Trick $\left.\right|^{6}$ was substituted for the General Valve solenoid-type molecular beam valve that was used in the carbon cluster studies, the $\mathrm{Si}_{4}{ }^{-}$ clusters were found to be dramatically cooled. The piezoelectric valve gives a sharper rise time on the pulse output, which apparently has a beneficial effect on cluster cooling- presumably because the ions have higher pressure carrier gas thrusting the plasma into the clustering 
channel and throughout the subsequent expansion into the chamber. The source configuration found to generate the vibrationally coldest $\mathrm{Si}_{3}{ }^{-}$and $\mathrm{Si}_{4}^{-}$spectra without further source moditications is again that shown in Figure (2.2).

Silicon clusters larger than the tetramer were found to have a nearly undetectable photodetachment cross section near threshold, precluding our obtaining ZEKE spectra for these three dimensional species.

\section{B. Liquid nitrogen-cooled clustering channel}

With the source showr, in Figure (2.2) used in conjunction with the piezoelectric valve, the ZEKE spectra of the indium phosphide triatomics (Chapter 9) were also successfully obtained. The larger clusters, however, were problematic in that the detachment signal could be observed, but no structure was resolved. This was the impetus to modify the source to provide even more efficient cluster cooling by cooling the clustering channel with liquid nitrogen (although it ultimately did not result in acquiring ZEKE spectra of larger clusters).

The design of the $\mathrm{N}_{2 ;}$, cooled source was made after considering sources made by $\mathrm{Chen}^{7}$ and Bloomfield, ${ }^{8}$ and is shown in Figure (2.3). It is qualitatively similar to the roomtemperature source described above, but the main differences are as follows. The clustering channe! is a much longei piece (1.3") made from copper, has a smaller inner bore $\left(0.1^{\prime \prime}\right)$, and is terminated with a $0.0625^{n} 1 D$ exit slit made from brass. The source piece is made from Vespel to thermally insulate the molecular bearn valve from the cooled channel, and also has an inner bure of $0.1^{*}$. Pieces of mica are placed between the contacting surfaces of the various pieces for further thermal insulation. A 1/8" copper tube carrying $\mathrm{N}_{2(0)}$ from a chicken feeder located outside of the vacuum chamber is fastened directiy to the channel. The whole chicken feeder setup is floated at $-1 \mathrm{kV}$, as is the rest of the source. Thermocouples attached to the channel and the source indicate a channel temperature of approximately 100 to $115 \mathrm{~K}$ at equilibrium. 
Even with the Vespel source piece and the mica insulating the beam valve from the cooled channei, some cooling of the beam valve still does occur. The General Valve molecular beam valve was found to be less sensitive to this cooling than was the o-ring tip of the piezoelectric valve, and is therefore used when $\mathrm{N}_{2(n)}$-cooling is implemented. Helium is used as the carrier gas, as it is the most thermally conductive of the rare gases.

With the $\mathrm{N}_{2(t)}$-cooled clustering channel, effective vibrational cooling of the clusters was only observed using certain timings between the molecular beam valve fing and the ablation laser firing. As mentioned above, in the room temperature configuration, the best cooling occurs when ablation roughly coincides with the early part of the rising edge of the molecular beam

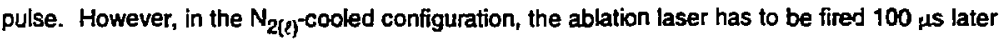
than it is fired in the room temperature configuration. This suggests that for good thermal contact to be made between the clusters and the cooled clusterirg channel, the clusters must encounter high pressure of already cooled helium that has preceded the plasma into the channel. The smaller exit slit allows tor the pooling of the helium in the channel. Without it, no enhanced cooling is observed.

Figure (2.4) shows a section of the $\mathrm{Si}_{4}{ }^{-}$ZEKE spectrum obtained using the piezoelertric beam valve used in conjuriction with the room temperaiure source (dotted line- see Chapter 7 ) and the liquid nitrogen-cooled source (solid line). It is clear that the sequence bands are markedly suppressed in the $\mathrm{N}_{2(y)}$-cooled cluster source.

\section{Deflectors added to the mass spectrometer}

A minor modification to the mass spectrometer that was found is enhance the cluster signal is a set of horizontal deflectors which were installed in the first differential region between the acceleration lens stack and the beam modulation plates. These deflectors compensate for any side-to-side deviation of the molecular beam off the axis of the apparatus (most likely due to 
a small amount of slack in the beam valve centering piece) before the ions travel more than several centimeters at $1 \mathrm{keV}$.

\section{Improvements to the detector region}

The detection region of the ZEKE apparatus is fundamentally the same as that described by Kitsopoulos, ${ }^{1}$ but a second set of laser windows has been added near the electron detector to accommodate total photodetachment cross section spectroscopy. A five-element Einzel lens for focusing the photoelectrons has replaced the previoushy employed three-element lens, and the magnetic shielding surrounding the flight path of the photoelectrons has been improved.

A diagram of the new detector region is shown in Figure (2.5). For this figure, in contrast to Figure (2.t), the detachment window flanges are oriented parallel to the plane and the photodetachment laser beam is thus perpendicular to the plane. The whole detector chamber was rebuilt using $8^{n} \mathrm{OD}$, stainless steel tubing rather than the previously used $6^{\prime \prime}$ tube, which is accompanied by the switch from $8^{n}$ to $10^{\prime \prime}$ contlat flanges. The same near-death Alcatel $450 \mathrm{Ls}^{-1}$ turbornolecular pump (TMP) backed by a Varian $80 \mathrm{Ls}^{-1}$ TMP maintains the larger chamber at a $3 \times 10^{-9}$ torr operating pressure. Some of the other gross structural changes with the new detector are as follows. The electron extraction plates (1) are mounted from upstream of the ion beam axis. There are only seven molybdenum plates, the last four of which are held at ground "I contrast to the 12 plates previously used. The first plate has a smaller hole: $0.5 \mathrm{~cm}$ compared to $0.5^{n}$ previously used. This is possible because the isn bearn is now definted by a 3 $\mathrm{mm}$ stainless steel knife-edged slit upon entering the detector region. The plate assembly is mounted onto an aluminum piece ( $\epsilon$ ) that keys into the first 6-way cross. A small tube of Hypemom (a), a magnetic shielding material similar to Mu-metal, also attaches to the aluminum mounting piece. Two other layers of Hypemom (b) and the electron Einzel lens (4) are attached 
to a stainless steel mounting piece that keys into the end of the second 6-way cross. The electron (5) and ion (6) detectors are roughly the same as in the previous design.

\section{A. Total photodetachment cross section windows}

The second set of detachment laser windows, mounted at Brewster's angle, allow for detachment of the negative ions immediately below the electron detector (5) in the electron and ion detection region IFigure (2.5); the Brewster's angle windows are shown from above schematically in Figure (2.1)]. This gives the apparatus the capability of collecting all of the photoelectrons as a function of photon energy when the ions are detached immediately adjacent to the electron detector. The information gained in this mode, which maps out the total photodetachment cross section of the anion, is useful for determining detachment threshold more precisely that the threshold determined from the lower-resolution photoelectron spectroscopy (PES, see Chapter 1, Sec. 1). It is also instrumental in the observation of transitions to excited anion states, if they exist, by means of two-photon detachment or autodetachment through the excited anion state. Such transitions appear as sharp features in the total photodetachment cross section, as they do in the case of $\mathrm{C}_{6}^{-}$(Chapter 3).

The main difficulty with these wincows was the excessive background noise resulting from stray photons impinging on the various intemal surfaces of the apparatus. The worst background electron signal occurred when an extensive set of anodized aluminum skimmer light baffles having small light holes and baffled tubes protruding close to the interaction region was used. Dramatically lower background levels were attained when anodized baftles having a kniiz-edged, $0.5 \mathrm{~cm}$ ID were situated within several $\mathrm{cm}$ of the windows, and a larger, $1 \mathrm{~cm}$ ID baffle was placed flush with the window flange. No baffles protruding slose to the interaction region are now used. However, background electrons are still problematic at wavelengths shorter than $270 \mathrm{~nm}$. 


\section{B. Five-element Einzel lens}

The five-element Einzel lens adds new flexibility to the focusing of the photoelectrons. The lens is generally run in decelerating mode, so the electrons move more slowly through the elements having some negative voltage applied. The first and last elements are always grounded, but the middle three elements can be connected in any way, as each is individually connected to separate feed-thrus. For instance, all three can be shorted together to provide the longest path of low electron kinetic energy, thereby giving a good deal of time for the energetic electrons to separate away from the threshold electrons. In such a case, a typical operating voltage is -0.6 to $-0.7 \mathrm{~V}$ when the electrons have around 2 to $3 \mathrm{eV}$ kinetic energy from the extraction. The temporal separation of the on-axis energetic electrons from the ZEKE's is dramatically improved operating in this mode: Rather than the 15 to $20 \mathrm{~cm}^{-1}$ electrons arriving at the detector $\mathbf{5 0} \mathrm{ns}$ prior to the ZEKE electrons as they typically did in the previous design, the separation has improved to 300 to 400 ns.

\section{Improved magnetic shielding}

Because the trajectories of low kinetic energy electrons are very sensitive to any stray fields (electric or magnetic) it is excruciatingly important that the inside of the detector region be made field-free. Stray electric fields from patch potentials on the surfaces of the electron aptics are (usually) effectiveiy eliminated by copious cleaning in addition to a thin layer of graphite sprayed on the surface. Stray magnetic fieids can come from the earth's magnetic field, which is on the order of 0.5 Gauss (not necessarily perpendicular to the ion beam axis of the apparatus, however) and the stainless steel surfaces in the apparatus. The main objective of the modifications to the delector chamber revolve around having more effective magnetic shieiding. 
The previous shielding was composed of two concentric cylinders of $0.02^{n}$-thick Hypemom having $4.5^{\prime \prime}$ and $5.5^{\prime \prime}$ diameters. These cylinders were divided into two sections; an approximately $5^{n}$-long section shielding the electron extraction region, and a $17^{m}$-long section shielding the electron Einzel lens. There was, however, a 1" gap between the extraction and Einzel shields. The new design allows for continuous shielding from the detachment region to actual detection, with triple layers of Hypemom shielding the detachment region where the electrons have the least kinetic energy and are most susceptible to stray fields.

The shielding offered by a single cylinder of some high-permeable material goes as ${ }^{9}$

$$
S_{i}=\mu_{i} t_{i}^{\prime} / 2 R_{i}
$$

where $\mu_{i}$ is the permeability, $t_{i}$ is the thickness of the sheet, and $R_{i}$ is the radius of the cylinder. For concentric cylinders, the shielding improves multiplicatively:

$$
S=1+S_{1}+S_{2}+\ldots+S_{1} S_{2}\left(1-A_{1} / A_{2}\right) S_{3}\left(1-A_{2} / A_{3}\right) \ldots
$$

In this equation, $A_{i}$ is the surface area of the cylinders. We can compare the shielding provided by the new design and the old given an approximate permeability of 20,000 for Hypemom (this value, from the CRC Handbook, was for 20 Gauss. The actual value will be lower in a smaller magnetic field). In the old design, $t_{i}=0.02^{\prime \prime}, R_{1}=2.25^{\prime \prime}$, and $R_{2}=2.75^{\prime \prime}$, giving $S=1332$ in the interaction region. In the new design, $t_{i}=0.02^{\prime \prime}, R_{1}=2.25^{n}, R_{2}=2.675^{\prime \prime}$, and $R_{3}=3.25\left(L_{2}=L_{3}\right.$ $=4 L_{1}$ ), giving $S=54,000$. This 40 -fold improvement in shielding may ultimately make the traditionally temperamental ZEKE apparatus run more reliably. 
1 T. N. Kitsopoulos, I. M. Waller, J. G. Loeser, and D. M. Neurnark, Chem. Phys. Lett. 159, 300 (1989); T. N. Kitsopoulos, C. J. Chick, Y. Zhao, and D. M. Neumark, J. Chem. Phys. 95,1441 (1991); T. N. Kitsopoulos, Ph.D. Thesis, University of California. Berkeley (1991).

2 T. G. Dietz, M. A. Duncan, D. E. Powers, and R. E. Smalley, J. Chem. Phys. 74, 6511 (1981).

3 J. M. B. Bakker, J. Phys. E 6785 (1973); J. Phys. E. 7, 364 (1974).

${ }^{4}$ K. Müller-Dethlefs, M. Sander, and E. W. Schlag, Z. Naturtorsch, 39A, 1089 (1984); Chem. Phys. Lett. 12, 291 (1984); K. Müller-Dethlefs and E. W. Schlag, Ann. Rev. Phys. Chem. 42, 109 (1991).

5 T. N. Kitsopculos, C. J. Chick, Y. Zhao, and D. M. Neumark, J. Chem. Phys. 95, 5479 (1991).

${ }^{6}$ D. Proch and T. Trick, Rev. Sci. Instrum. 60, 713 (1989).

7 D. W. Kohn, H. Clauberg, and P.Chen., Rev. Sci. Instrum, 63, 4003 (1992).

8 J. P. Bucher, D. C. Douglass, and L. A. Bloomfie d, Rev. Sci. Instrum, 63. 5667 (1992).

${ }^{9}$ R. D. Vance and W. F. Griffith, Electronic Packaging Production, October, 1978. 
Figure (2.1) Schematic of the threshoid photodetachment (ZEKE) apparatus.

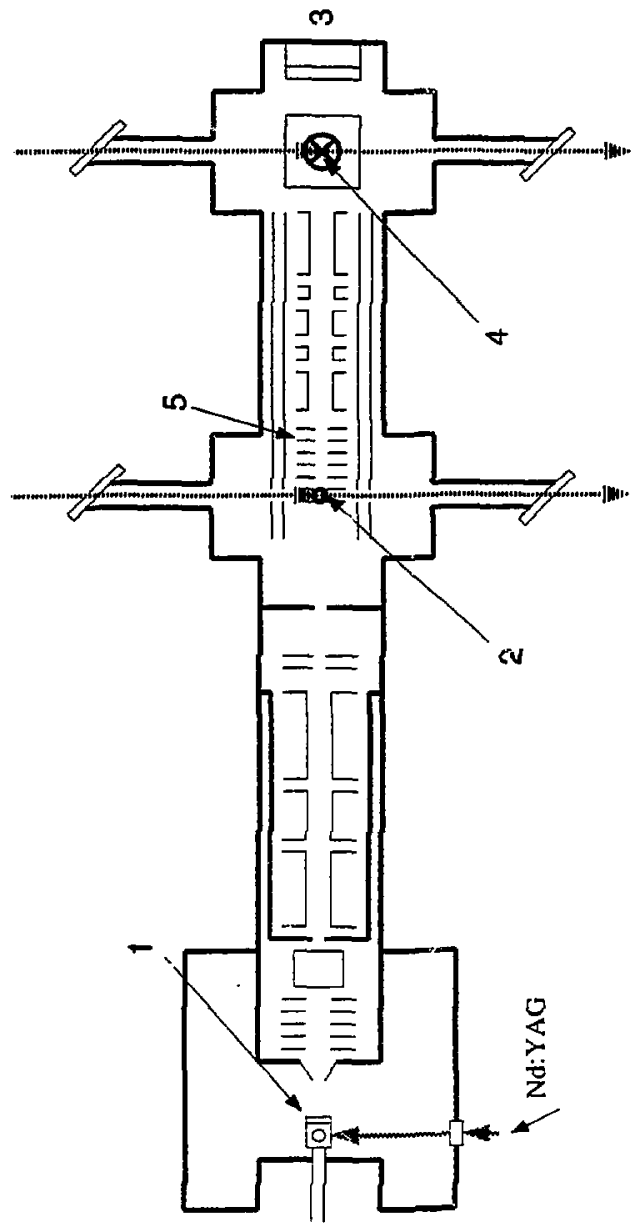


Figure (2.2) Room temperature cluster source.
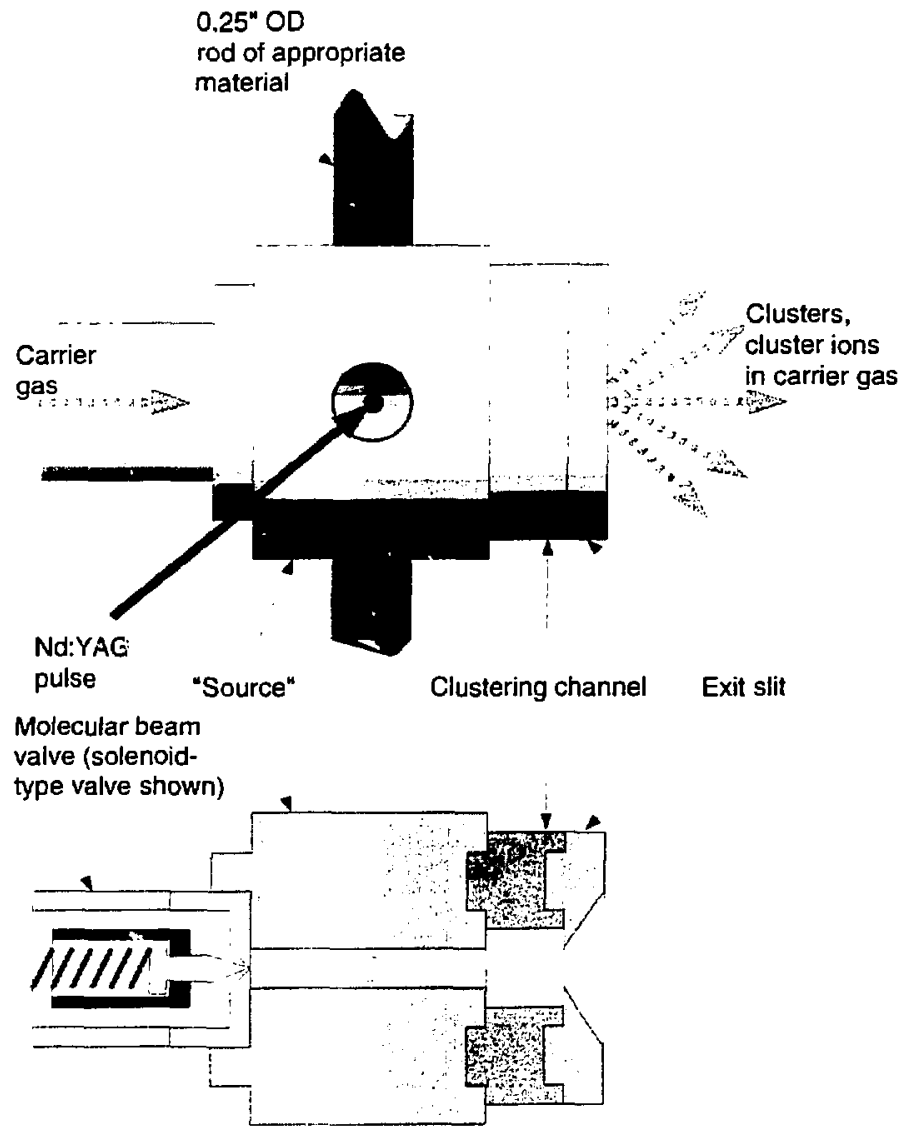
Figure (2.3) Liquid nitrogen-cooled clustering channel.

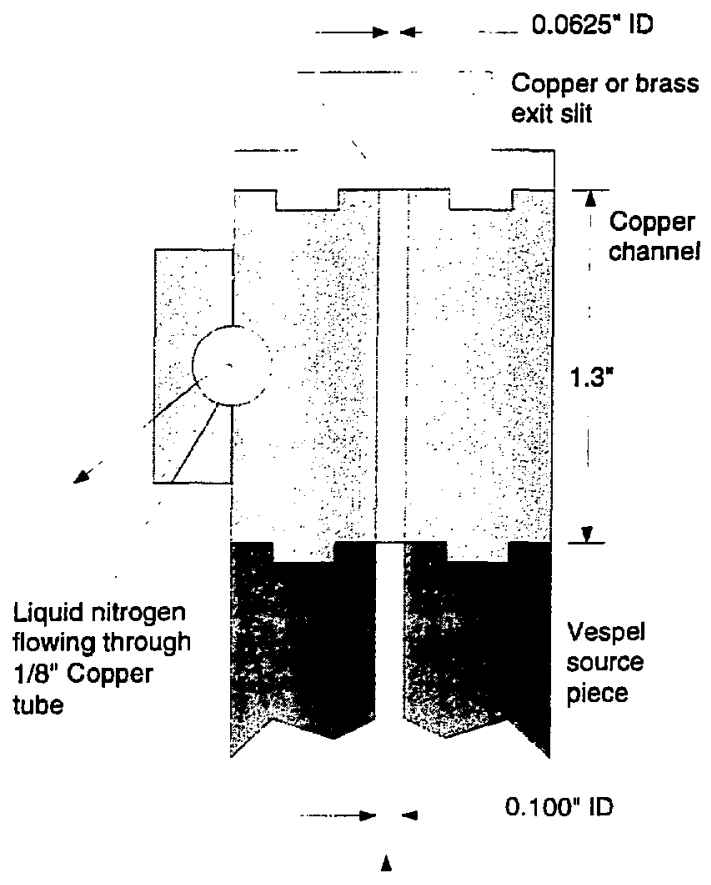

Molecular beam

direction 
Figure (2,4) Comparison of $\mathrm{Si}_{4}-$ ZEKE spectra obtained using the room-temperature cluster source (dotted line) and the liquid nitrogen-cooled cluster source (solid line).

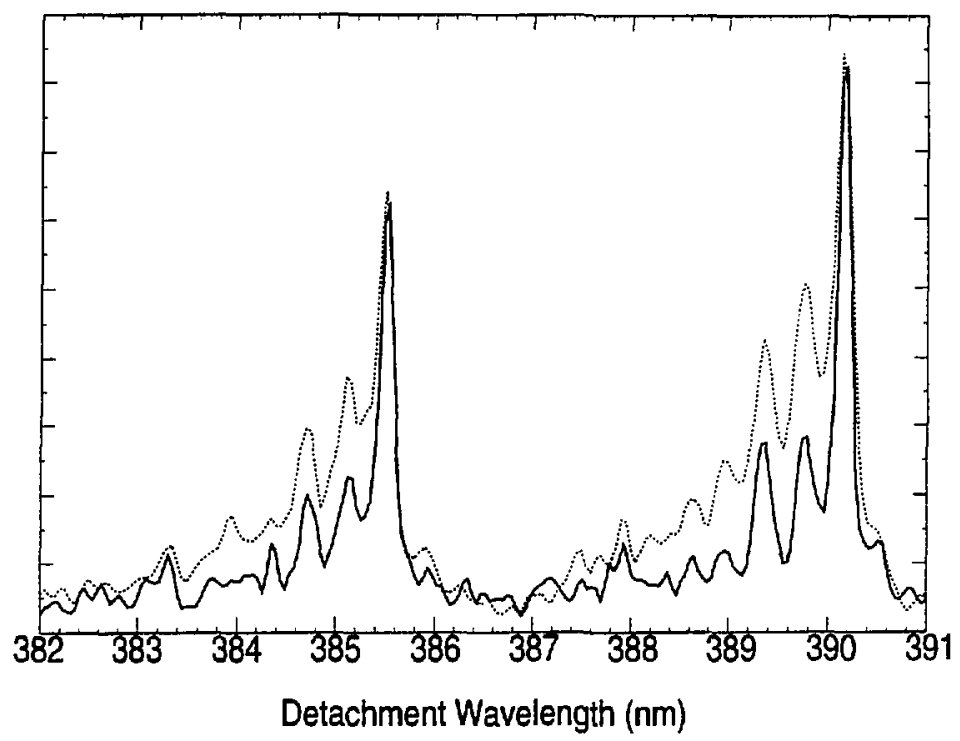


ZEKE detachment

and delayed extraction

Electron and ion

region

detection region

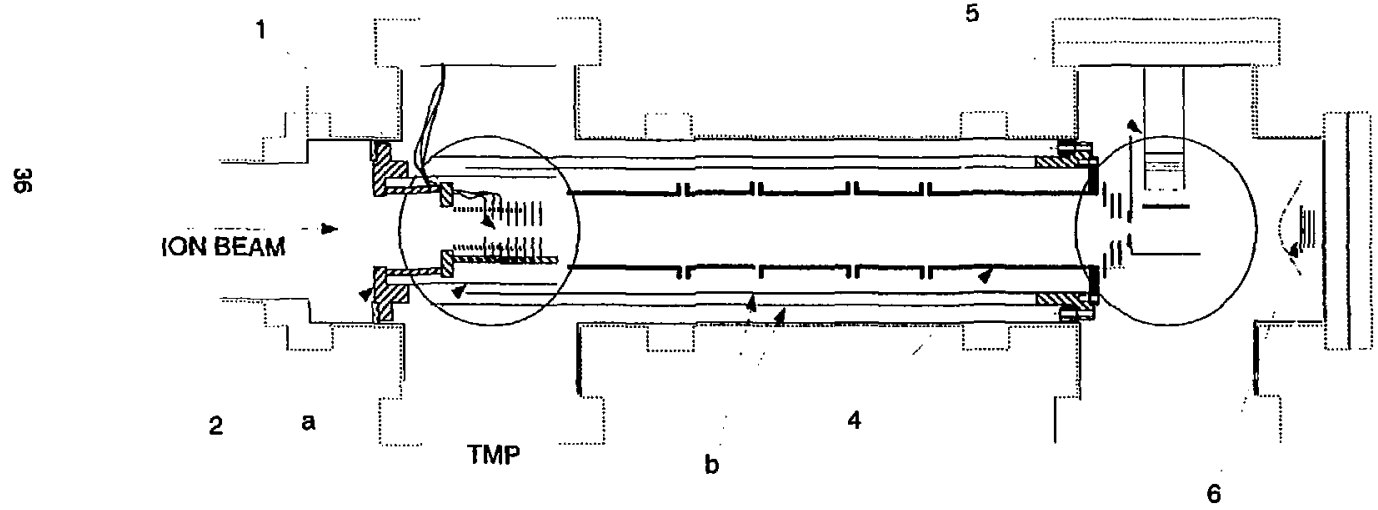

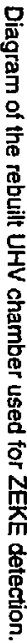




\title{
Chapter 3. Study of $\mathrm{C}_{6}-$ and $\mathrm{C}_{6}$ with threshold photodetachment spectroscopy and autodetachment spectroscopy
}

\author{
Abstract
}

The $\mathrm{C}_{6}{ }^{-}$anion and $\mathrm{C}_{6}$ neutral have been studied using both threshold photodetachment (ZEKE) spectroscopy and autodetachment spectroscopy of $\mathrm{C}_{6}-$. The threshold photodetachment spectrum yields the electron affinity of linear $C_{6}$ to high accuracy, along with the three symmetric stretch frequencies for linear $\mathrm{C}_{6}$ and the spin-orbit splitting in the ground ${ }^{2} \mathrm{TI}_{\mathrm{U}}$ state of the anion. Two of the symmetric stretch frequencies are significantly lower than previous ab initio predictions. A simple model force field is used to calculate stretching force constants and estimate bond length changes between the anion and neutral. In addition, using autodetachment spectroscopy, we have located an excited electronic state of $\mathrm{C}_{6}{ }^{-}$that lies 43 $\mathrm{cm}^{-1}$ below the detachment threshold. This state is very similar in geometry to neutral $\mathrm{C}_{6}$. Excited vibrational levels of this state autodetach with rates that depend strongly on the available autodetachment channels. The excited state is tentatively assigned to a valence state, rather than an electrostatically bound state. 


\section{Introduction}

Elemental carbon clusters have been the subject of considerable experimental and theoretical research because of their importance in combustion ${ }^{1}$ and astrophysics. ${ }^{2}$ The state of carbon cluster research up to 1989 is described in an excellent review article by Weitrer and Van Zee. ${ }^{3}$ Prior to 1989 , most of our knowledge regarding the structure and spectroscopy of small carbon clusters was from ab initio calculations. Experimentally, rotationally resolved spectra had only been obtained for $\mathrm{C}_{2}{ }^{4}$ and $\mathrm{C}_{3},{ }^{5}$ results for the larger carbon clusters (4-10 atoms) were limited to several matrix isolation studies $6,7,8$ and a photoelectron spectroscopy study of carbon cluster anions. ${ }^{9}$ However, during the last three years, new experiments have dramatically increased our understanding of the spectroscopy and structure of these species. For example, Saykally and co-workers ${ }^{10}$ have measured gas phase, rotationally resolved infrared spectra for $\mathrm{C}_{3}, \mathrm{C}_{4}, \mathrm{C}_{5}, \mathrm{C}_{6}, \mathrm{C}_{7}, \mathrm{C}_{9}$ and $\mathrm{C}_{93}$, yielding rotational constants and vibrational frequencies; Amano, Bemath, and co-workers ${ }^{11,12}$ have also obtained high resolution spectra for several of these species. All of the species observed in these gas phase studies appear to be linear.

Negative ion photodetachment provides another poweriul experimental probe of carbon clusters. Although these experiments are typically lower resolution than infrared absorption, they offer several important advantages. One can mass select the anion prior to spectroscopic investigation, eliminating any ambiguity concerning species identification. This is important in cluster studies as most cluster sources simultaneously generate a number of different species (anions, neutrals and cations). In addition, photodetachment tends to result in excitation of totally symmetric vibrational modes of the neutral. With sufficient resolution, one can determine the frequencies of these modes. Since these are no: infrared active for linear molecules, the information obtained is complementary to infrared absorption experimerts. 
Photoelectron spectra of the carbon cluster anions $C_{n}-(n=2$ through 29$)$ hive been obtained by Smalley and roworkers, ${ }^{9}$ and considerably higher resolution spectra $\left(60-100 \mathrm{~cm}^{-1}\right)$ have heen measured is $\mathrm{C}_{2}-$ and $\mathrm{C}_{3}-$ by Lineberger, ${ }^{13.14}$ and for $\mathrm{C}_{2}-$ through $\mathrm{C}_{11}-$ by our group. ${ }^{15}$ These studies show that the linear even clusters have higher electron affinities than the odd clusters, in agreement with the prediction of Pitzer and Clementi. ${ }^{16}$ The higher resolution studies also yielded numerous vibrational frequencies for the neutral clusters. Finally, we have studied $\mathrm{C}_{5}{ }^{-}$at even higher resolution using threshold photodetachment spectroscopy. ${ }^{17}$ The resolution of this technique is $3-8 \mathrm{~cm}^{-1}$, and the resulting spectrum shows wellresolved transitions to various symmetric stretch and bending modes of $\mathrm{C}_{5}$, as well as fine structure due to the spin-orbit splitting in the anion.

This paper describes new experiments on the photodetachment spectroscopy of $\mathrm{C}_{6}{ }^{-}$. the goal of which is to leam about the structure and spectroscopy of $C_{6}$ and $C_{6}-$. Several calculations have been performed on neutral $C_{6}$ predicting close-lying cyclic ${ }^{1} A_{1}{ }^{\prime}\left(D_{3 n}\right)$ and ${ }^{3} \Sigma_{g}-$ $\left(D_{\infty h}\right)$ linear states, although the true ground state remains a point of controvers $y .{ }^{18,79}$ Raghavachari ${ }^{18}$ and Martin ${ }^{20}$ have calculated vibrational frequencies for the cyclic and linear isomers, respectively. Calculations performed on the anion have predicted a linear ${ }^{2} \Pi_{4} G \cdot$ ound state. ${ }^{21}$ Adamowicz ${ }^{22}$ has predicted the existence of several low-lying electronic states of linear $\mathrm{C}_{6}-$.

Experimentally, several intrared abrorption studies have been performed on $C_{6}$ in a low temperature, rare gas matrix. ${ }^{6,23}$ The assignment of the observed lines to $C_{6}$ antisymmetric mode has been problematic; only recently, with extensive isotopic substitution, has a line at $1952 \mathrm{~cm}^{-1}$ been definitively assigned to a $C_{6}$ antisymmetric, stretch. Electron spin resonance (ESR) studies ${ }^{24}$ in rare gas matrices have shown than linear $C_{6}$ in a ${ }^{3} \Sigma$ state exists, and that the bonding is cumulenic, in accordance with the prediction of Ref. (16). Experimental information on the anion comes from the aforementioned photoelectron spectroscopy studies.9,15 In addition $i$ sielding the electron affinity of linear $C_{6}, 4.185 \pm 0.006 \mathrm{eV}_{1}^{15}$ the main features in 
these spectra were assigned to transitions between linear $\mathrm{C}_{6}{ }^{-}$and $\mathrm{C}_{6}$. However, a high energy 'ail' in our $\mathrm{C}_{6}-$ spectrum was tentatively attributed to a high energy cyclic isomer of the anion. Coulomb explosion measurements also suggest fine existence of a cyclic $\mathrm{C}_{6}{ }^{-}$isomer. ${ }^{25}$

The results reported he:e in $\mathrm{C}_{6}-$ are from two techniques: negative ion threshold photodetachment spectroscopy (zero electron kinetic energy, or ZEKE spectroscopy) and autodetachment spectroscopy. Figure (3.1) illustrates the energetics of both techniques. In threshold photodetachment spectroscopy, the anions are photodetached with a tunable laser, and the near-zero kinetic energy electron signal is collected as a function of laser frequerivy. These threshold electrons are produced only when the laser is resonant with an anion $\rightarrow$ neutral transition:

$$
C_{6}^{-}\left(v^{\prime \prime}\right)+h v \rightarrow C_{6}\left(v^{\prime}\right)+e^{-}(\text {eKE }=0)
$$

By this means, we can map out the direct photodetachment transitions between the $C_{6}{ }^{-}$ground state and various vibrational (and electronic) levels of neutral $C_{6}$ with $3 \mathrm{~cm}^{-1}$ resolutlun.

In conurast, autodetachment spectroscopy 26 is sensitive to transitions between the ground and excited electronic states of the anion. The autodetachment spectrum of $\mathrm{C}_{6}-$ is obtained by measuring the total photodetachment cross section as a function of the detachment laser frequency. In the absence of anion excited states (the usual case), the photodetachment cross section rises monotonically above the detachment threshold, although in favorable cases one can observe steps in the cross section when new anion $\rightarrow$ neutral transitions become energetically accessible. ${ }^{27}$ However, it there are long-lived excited states of the anion, $\left(\mathrm{C}_{6}-\right)^{*}$. just above the detachment threshoid, the photodetachment cross section will exhibit sharp Feaks resulting from excitation to the metastable $\left(\mathrm{C}_{6}-\right)^{*}$ state followed by autodetachment to the neutral plus electron: 


$$
C_{6}^{-}+h v \rightarrow\left(C_{6}^{-}\right)^{*} \rightarrow C_{6}+e^{-}(e K E \neq 0)
$$

The combination of threshold photodetachment spectroscopy and autodetachment spectroscopy has been applied previously to $\mathrm{Au}_{2}{ }^{-}$and $\mathrm{Au}_{6}-$ by Kaldor and coworkers. 28,29

The threshold photodetachment results presented below show well-resolved transitions between linear $\mathrm{C}_{6}{ }^{-}$and $\mathrm{C}_{6}$, and yield the symmetric stretch frequencies for $\mathrm{C}_{6}$. In addition, the photodetachment cross section results show sharp autodetachment peaks, indicating that there is a excited electronic state of $\mathrm{C}_{6}$ - near the detachment threshold. The vibrational frequencies of this anion state are very similar to those of neutral $\mathrm{C}_{6}$. While $\mathrm{C}_{2}-$ is known to undergo autodetachment, ${ }^{26}$ our results are the first such observation for a polyatomic carbon cluster anion.

\section{Experimental}

Figure (3.2) shows a schematic top view of the apparatus employed for all of the experiments reponed in this paper. The machine is described in detail eisewhere, ${ }^{30}$ but the basic operation is as follows. Cold carbon clusters (anions, cations, and neutrals) are generated in a laser vaporization'puised molecular beam source similar to that developed by Smalley. ${ }^{31}$ Helium is used as the carrier gas, typically with a backing pressure of 90 psi. Laser vaporization is achieved by focussing a $4 \mathrm{~mJ}, 532 \mathrm{~nm}$ pulse from a frequency-doubled $\mathrm{Nd}$ :YAG laser $(20 \mathrm{~Hz}$ repetition rate) onto a rotating, translating graphite rod (higher pulse energies resulted in significantly hotter ions). The negative ions that pass through a $2 \mathrm{~mm}$ diameter skimmer are collinearly accelerated to $1 \mathrm{keV}$. Mass selection is achieved with a $1 \mathrm{~m}$ long beam-modulated time-of-flight mass spectrometer. 32 The mass-separated arions then ener the detection region where they are photodetached by a (pulsed) excimer-pumped dye laser in either of two locations: $60 \mathrm{~cm}$ upstream of the ion and electron detectors [the region labelled " $A$ " in Figure 
(3.2)] or adjacent to the ion and electron detectors in the region labelled "B" in Figurc (3.2). For photodetachment of linear $\mathrm{C}_{6}{ }^{-}$, the dye laser output was doubled with a BBO crystal. The dyes used were Rhodamine 600 (300 to $310 \mathrm{~nm}$ ), Rhodamine 590 perchlorate $(287$ to $300 \mathrm{~nm}$ ) and Coumarin 540 (270 to $289 \mathrm{~nm})$.

Three types of photodetachment experiments can be performed with this instrument. We can obtain threshold photodetachment spectra and "partially discriminated" photodetachment cross sections by photodetaching the anions at " $A$ ". Total photodetachment cross sections are obtained by photodetachment at "B", directly below the electron detector [marked with the in Figure (3.2)]. In all three experiments, the electron signal is nomalized with respect to the ion current and laser power. Each mode of operation will now be described in more detail.

Threshold photodetachment spectra are obtained by adapting the zero electron kinetic energy (ZEKE) spectroscopy method developed by Müller-Dethleis et al..$^{33}$ to negative ion photodetachment. In these experiments, $\mathrm{C}_{6}-$ clusters are photodetached in region $\mathrm{A}$. The ejected photoelectrons are extracted with a pulsed field of about $2 \mathrm{~V} / \mathrm{cm}$, which is applied $200 \mathrm{~ns}$ after the photodetachment laser pulse. This delay allows the higher energy electrons to separate spatially from the threshold electrons that have nearly zero kinetic energy. Once the field is applied. the energetic electrons with significant velocity perpendicular to the ion beam direction are prevented from reaching the detector by a series of apertures between the extraction region and electron detector. Threshold electrons and higher energy electrons scattered along the ion beam axis pass through a $60 \mathrm{~cm}$ long Einzel lens and are then deflected into the electron detector with a weak field. While these higher energy electrons cannot be discriminated against by the apertures, they emerge from the extraction region with different kinetic energies than the threshold electrons, due to the spatial separation along the beam axis that occurs prior to applying the extraction pulse. The higher energy electrons can then be discriminated against with a gated detection scheme, in which only those electrons arriving at 
the detector within a $35 \mathrm{~ns}$ window are counted. This combination of spatial and temporal discrimination yields an electron kinetic energy resolution of $3 \mathrm{~cm}^{-1}$. The resulting peak for an ideal atomic system (one which undergoes s-wave detachment near threshold ${ }^{30.34}$ ) is shown in Figure (3.3a).

If the $\mathrm{C}_{6}{ }^{-}$clusters are photodetached at $\mathrm{A}$ and the extraction pulse is applied with no delay, one obtains a "partially discriminated" photodetachment cross section scan. The electron extraction field immediately accelerates all of the photoelectrons toward the electron detector. However, those photoelectrons produced with a significant perpendicular velocity component are still spatially discriminated against by the apertures en route to the electron detector. This is therefore a variation on the "steradiancy detector" described many years ago by Spohr et al. ${ }^{35}$ and Baer $e t$ al. ${ }^{36}$ The result is an asymmetric peak which is about $150 \mathrm{~cm}^{-1}$ wide [Figure (3.3b)]; the collection efficiency drops off dramatically for electrons produced with more than $150 \mathrm{~cm}^{-1}$ kinetic energy. Hence, scans performed in this mode of operation are referred to as "partially discriminated" photodetachment cross section scans.

Finally, to measure the totai photodetachment cross section, the clusters are photodetached via the second set of laser windows at region 8 . which lies just below the electron detector. All of the photoslectrons are collected with a $10 \mathrm{~V} / \mathrm{cm}$ extraction field. The resulting cross section for an ideal atomic system (s-wave photodetachment) will resemble the spectrum in Figure (3.3c).

If direct photodetachment is all that is occurring, there is little reason to pertorm anything other than threshold photodetachment spectroscopy with this instrument. However, electrons resulting from autodetachment processes will often have considerably more than $3 \mathrm{~cm}^{-1}$ kinetic energy and will therefore not be observed in a threshold photodetachment spectrum, so the instrument must be operated in one of the other two modes to study autodetachment. In the "partially discriminated" mode of operation, only relatively low Eilergy electrons from autodetachment will be collected efficiently, while virtually all the photoelectrons produced by 
autodetachment will be collected in the total cross section mode. Differences in the intensity of autodetachment peaks cbtained in these two modes can serve as a qualitative measure of the electron kinetic energy resulting from autodetachment.

In addition to the above experiments, photodetachment power studies and two-color, wo-photon photodetachment scans are reported in this paper. These are done in order to ascertain the importance of single photon vs. multiphoton detachment for the various peaks observed in tie total photodetachment cross section. The photodetachment power studies entail counting electrons produced in region B as a function of laser pulse energy for a particular detachment wavelength. The electrons are counted for 5000 to 6000 laser shots at a given laser power and normalized to the collected ion current. In the two-color, two photon scans, $\mathrm{C}_{6}$ is photodetached at " $\mathrm{B}$ " by copropagating, simultaneous laser pulsts from the doubled dye laser and the second harmonic of a Nd:YAG laser $(532 \mathrm{~nm})$. The two laser pulses are made simultaneous with the aid of a photodiode. For these experiments, the dye laser fluence vits 25 $\mathrm{mJ} / \mathrm{cm}^{2}\left(0.5 \mathrm{~mJ} /\right.$ pulse) and the $532 \mathrm{~nm}$ fluence was $100 \mathrm{~mJ} / \mathrm{cm}^{2}(2 \mathrm{~mJ} / \mathrm{pulse})$. Any enhancement of the photodetachment signal by the YAG laser provides strong evidence for two photon processes (see below).

\section{Results}

Figures (3.4a, $b$, and $c$ ) show the results of the three types of scans discussed in the experimental section for $\mathrm{C}_{6}-$. Figure $(3.4 \mathrm{a})$ shows the threshold photodetachment spectrum, Figure (3.4b) shows the partially discriminated cross section, and Figure (3.4c) shows the total photodetachment cross secton.

The threshold photodetachment spectrum is dominated by peak A located at $296.65 \mathrm{~nm}$. There are also three irregularly spaced peaks with approximately one third the intensity of peak A at higher energies. The positions and relative energies of these peaks, labelled D. G and $\mathrm{I}$, 
are listed in Table (3.1). A less intense peak is observed $29 \mathrm{~cm}^{-1}$ to the red of each of these peaks; these smaller peaks are labelled $a, d, g$ and $i$. All of the peaks in Figure (3.4a) are approximately $17 \mathrm{~cm}^{-1}$ wide (FWHM). The rising baseline toward higher photon energies is due to background photoelectrons generated by stray photons impinging on various metal surfaces in the extraction region.

In the partially discrimınated cross section scan [Figure (3.4h)], the direct photodetachment transitions [Eqn. (3.1)] are evident as distinct thresholds, which are at the same wavelengths as the peaks seen in the threshold photodetachment spectrum. These thresholds are also labelled A, D, G and I. The electron signal near each threshold has the general appearance of the model spectrum in Figure (3.3b), rising sharply then decreasing gradually in intensity as the photon energy increases. In addition, a series of sharp peaks are observed which do not appear in Figure (3.4a). These are due to transitions to excited states of the anion followed, in most cases, by autodetachment [Eqn. (3.2)]. Four manitolds of these peaks tie just to the red of the four di ect detachment thresholds and are labelled SA, SD, SG and $\mathrm{SI}$ in reference to the thresholds to which they correspond. There are also two fairly intense individual peaks $\left(b_{0}\right.$ and $e_{0}$ ) that to not appear to correspond to direct photodetachment thresholds.

In the total photodetachment cross section scan [Figure (3.4c)] the direct detachment thresholds, again labelled A, D, G and I, are considerably less distinct than in Figure (3.4b). On the other hand, the autodetachment peaks are more intense relative to the direct detachment thresholds. The most intense peaks in each of the four manifrids SA. SD, SG, and SI. are labelled $a_{0}, d_{0}, g_{0}$, and $i_{0}$. In addition, several sharp but less intense autodetachment peaks not observed in the partially discriminated scan appear in the total cross section scan: these are labelled $b_{0}, c_{0}$, etc. In both Figures (3.4b) and (3.4c), the SA manifold does not dominate over the other sharp peak manifolds as peak $A$ in Figure (3.4a) dominates over the rest of the peaks. However, the SD, SG and SI manifolds in the total cross section scan have comparable 
intensities, while in the partially discriminated scan, they decrease in intensity toward the higher photon energies. The positions and relative energies of the labelled peaks are listed in the first three columns of Table (3.2). Note that peak $m_{0}$ is not shown in Figure (3.4c), but it has approximately the same intensity as peak $k_{0}$. Also, there are several broad peais in Figure (3.4c) which have not been assigned.

Figures $(3.5 a, b)$ and $(3.6 a, b)$ show expanded scale, finer step scans of the SA and SB manifolds, respectively. Figures (3.5a) and (3.6a) show the partially discriminated cross section, while Figures (3.5b) and (3.6b) show the total cross section. These figures reveal that these manifolds consist of several peaks of 5 to $7 \mathrm{~cm}^{-1}$ FWHM , many of which are actually partially resolved doublets with a characteristic splitting of $-1.7 \mathrm{~cm}^{-1}$. This splitting is most likely due tc the contours of unresolved rotational transitions. The rotational constant for $\mathrm{C}_{6}{ }^{-}$is expected to be around $0.04 \mathrm{~cm}^{-1}$, so individual rotational transitions cannot be observed at our current resolution $\left(0.4 \mathrm{~cm}^{-1}\right)$. This splitting does not appear in peaks salonging to the higher energy SG and SI manifolds. Table (3.3) lists the peak positions and relative energies for Figure (3.5b). For those peaks which appear as doublets, the peak positions and relative energies refer to the center of the doublet.

The total cross section and partially discriminated scans of the the SA manifold at 297 $\mathrm{nm}$ are very different. The most intense feature in Figure (3.5b) is the $\mathrm{a}_{0}$ doublet at $297.019 \mathrm{~nm}$. This is almost completely missing in the partially discriminated scan, and implies that the corresponding electrons are highly energetic. Note that the largest peak in the SA manifold in Figure $(3.4 b)$ is $a_{1}$, not $a_{0}$. The $a_{2}$ peak and the $a_{3}$ doublet in Figure (3.5b) are also virtually absent in Figure (3.5a). The differences between Figures (3.6a) and (3.6b) are not quite as dramatic. The most intense feature in the total cross section scan, the $d_{0}$ doublet at $292.84 \mathrm{~nm}$, is much smaller relative to the other features in the partially discriminated scan.

Figures (3.7a) and (3.7b) show total cross section scans from 292 to $298 \mathrm{~nm}$ under two different ion source conditions. The He backing pressure in Figure ( $3.7 \mathrm{a})$ is considerably higher, 
so the vibrational temperature in Figure (3.7a) should be lower than in Figure (3.7b). The data show that the intensities of peaks $a_{0}$ and $d_{0}$ are largely unaffected by source conditions, but the features just to the red of each of these peaks are more intense in Figure (3.7b). These features are aftributed to sequence bands from vibrationally excited $\mathrm{C}_{6}{ }^{-}$and are discussed in more detail below.

Figure (3.8) shows a log-log plot of the electron signal (in the total cross section mode) vs. photodetachment laser pulse energy at $297.026 \mathrm{~nm}$ and $297.186 \mathrm{~nm}$. These wavelengths correspond to the more intense peaks of the $a_{0}$ and $a_{1}$ doublets. respectively. The slope of the $a_{0}$ curve is approximately 1 while that of the $a_{1}$ curve is 0.5 , indicating that the $a_{0}$ peak intensity depends linearly on laser pulse energy, while the $a_{1}$ intensity varies as the square root of the pulse energy. Hence, the $a_{1}$ transition appears more strongly saturated than the $a_{0}$ transition.

Figures (3.9a) and (3.9b) show the results of the two-color, two-photon scans pertormed on $\mathrm{C}_{6}-$ for the SA manifold and part of the SD manifold, respectively. These scans are taken in the total photodetachment cross section mode and show the effect of an additional, simultaneous $532 \mathrm{~nm}$ pulse on the efectron signal. The results are superimposed on scans obtained under the same anion conditions and tunable dye laser power, but with no $532 \mathrm{~nm}$ pulse. Many of the peaks in these two regions exhibit a change in intensity with the addition of the $532 \mathrm{~nm}$, but the most dramatic effect occurs for the $297.019 \mathrm{~nm}$ doublet $\left(\mathrm{a}_{0}\right)$; its intensity increases by a factor of three in the presence of both laser pulses. Almost no change in intensity was observed for the $297.179 \mathrm{~nm}$ doublet $\left(a_{1}\right)$. The changes are less dramatic in Figure (3.9b); the intensity cf the largest feature, the $d_{0}$ doublet, increases by a factor of 1.6 in the two-color scan. The relative intensities of the peaks in the one-color and two-color scans are given in Table (3.4). 


\section{Analysis and Discussion}

\section{A. Threshold photodetachment spectrum}

\section{1) Peak assignments}

The $C_{6}{ }^{-}$threshold photodetachment spectrum is dominated by peak $A$; peaks $D, G$, and $I$ are about a factor of three smaller. We assign peak $A$ to the origin of the $C_{6} \leftarrow C_{6}-$ direct photodetachment transition, while the higher energy peaks are assigned to transitions to vibrationally excited $\mathrm{C}_{6}$ levels. The dominance of the origin transition indicates a small geometry change between the anion and neutral.

The doublet structure of the peaks of the threshold photodetachment spectrum strongly supports previous assignments of the $\mathrm{C}_{6}-$ photoelectron spectrum in this energy range to a transition between linear $\mathrm{C}_{6}{ }^{-}$and linear $\mathrm{C}_{6}$, as opposed to a transition between cyclic structures. The linear anion and neutral ground state term symbols are ${ }^{2} \Pi_{u}{ }^{21,22}$ and ${ }^{3} \Sigma_{g},{ }^{-16,19}$ respectively. The ${ }^{2} \Pi_{U}$ state is split into two spin-orbit components with $\Omega=3 / 2$ (the lower of the two) and $\Omega=$ $1 / 2$; if the splitting is small enough, both states will be significantly populated in the ion beam. Since the neutral ${ }^{3} \Sigma_{g}$ - state has no corresponding fine structure, each vibrational transition in the photodetachment spectrum should be a doublet, as we observe. The splitting in each doublet of $29 \mathrm{~cm}^{-1}$ is therefore the spin-orbit splitting $\mathrm{C}_{6}{ }^{-}$. For each doublet, the larger peak is from the $\Omega=$ $3 / 2$ level, and the smaller from the spin-ortit excited $\Omega=1 / 2$ level. The intensity ratio gives an electronic temperature of approximately $40 \mathrm{~K}$. For comparison, the spin-orbit splitting in the ${ }^{2} \Pi_{u}$ state of $\mathrm{C}_{5}-$ is $26 \mathrm{~cm}^{-1} .17$

The electron affinity of linear $C_{6}$ is given by the energy at which peak $A$ occurs, $4.180 \pm$ $0.001 \mathrm{eV}$. For comparison, the best previous experimental value, from our $\mathrm{C}_{6}{ }^{-}$photoelectron spectrum, was $4.185 \pm 0.006 \mathrm{eV} .{ }^{15}$ Ab initio studies have also predicted electron aftinities for linear $C_{6}$ in this range. 22,37 
We next consider the vibrational structure in our spectrum. In a photodetachment spectrum where the anion and neutral have different equilibrium geometries but the same overall symmetry, one expects progressions in totally symmetric vibrational modes of the neutral to dominate. ${ }^{38}$ Linear $\mathrm{C}_{6}$ has three totally symmetric modes (all symmetric stretches), and peaks $D, G$, and I are assigned to transitions to the fundamentals of the three modes. The resulting vibrational frequencies are $v_{3}=489 \pm 10 \mathrm{~cm}^{-1}$ (from peak D), $v_{2}=1322=10 \mathrm{~cm}^{-1}$ (peak G), and $v_{1}=2061 \pm 10 \mathrm{~cm}^{-1}$ (peak I). Our $v_{1}$ value is close to Martin's ab initio value of 2167 $\mathrm{cm}^{-1} .20$ However, our $v_{2}$ and $v_{3}$ frequencies are about 400 and $200 \mathrm{~cm}^{-1}$ lower, respectively, than the ab initio values. The experimental and calculated frequencies are summarized in Table (3.1).

Note that cyclic $C_{6}$ in $D_{3 h}$ symmetry has only two totally symmetric modes; the ab initio study by Raghavachari and Binkley ${ }^{39}$ predicts (scaled) frequencies of $1142 \mathrm{~cm}^{-1}$ and $800 \mathrm{~cm}^{-1}$ for these modes. Thus, the number of transitions to vibrationally excited states in our spectrum is consistent with a tinear $\leftarrow$ linear transition.

\section{2) $C_{6}$ force constants}

Since ab initio calculations overestimate the $v_{2}$ and $v_{3}$ frequencies, we have performed a simple, one-dimensional vibrational analysis on $\mathrm{C}_{6}$ in order to determine the force constants that would reproduce our observed frequencies. The most general one-dimensional harmonic potential in symmetrized internal coordinates is

$$
\begin{gathered}
V=\frac{1}{2}\left(k_{1} s_{1}^{2}+k_{2} s_{2}^{2}+k_{3} s_{3}^{2}+k_{4} s_{4}^{2}+k_{5} s_{5}^{2}\right)+k^{\text {antisym }} s_{4} s_{5}+k^{s y m, 1} s_{2} s_{3}, \\
+k^{s, m_{1}, 2} s_{1} s_{3}+k^{s y m, 3} s_{1} s_{2}
\end{gathered}
$$

where $s_{1}, s_{2}$, and $s_{3}$ are symmetric combinations of the internal coordinates $\left[s_{1}=2^{-1 / 2}\left(\Delta r_{a}+\Delta r_{e}\right)\right.$, $s_{2}=2^{-1 / 2}\left(\Delta r_{b}+\Delta r_{d}\right)$, and $\left.s_{3}=\Delta r_{e}\right]$ and $s_{4}$ and $s_{5}$ are antisymmetric combinations $\left[s_{4}=2^{-1 / 2}\left(\Delta r_{a}\right.\right.$ - 
$\left.\Delta r_{e}\right)$ and $\left.s_{5}=2^{-1 / 2}\left(\Delta r_{b}-\Delta r_{d}\right)\right]$. The internal coordinates and all of the couplings between them as given by Eqn. (3.3) are illustrated in Figure (3.10). Because this is a six-body problem confined to one-dimension, there is one translational degree of freedom and five vibrational degrees of freedom, corresponding to three symmetric stretches and two antisymmetric stretches. Therefore, in our attempts to reproduce our symmetric stretch frequencies, we will also generate two antisymmetric stretch frequencies.

As mentioned in the introduction, Vala and coworkers confirmed that a $1952 \mathrm{~cm}^{-1}$ IR line absorbed by carbon clusters trapped in rare-gas matrices is due to a $\mathrm{C}_{6}$ antisymmetric stretch $\left(v_{4}\right) \cdot 23$ A frequency close to this has also been assigned to the $v_{4}$ mode of $C_{6}$ in the gas phase in work done by Saykally and coworkers. 40 Years before, Thompson and coworkers attributed a $1197 \mathrm{~cm}^{-1}$ line to the lower frequency antisymmetric stretch $\left(v_{5}\right) .^{7}$ Most of the assignments made in that work have since been reassigned, but this particular line has neither been disputed nor confirmed as of yet. Both of these frequencies are in reasonable agreement with calculations; Martin predicts the two antisymmetric stretches to be $1244 \mathrm{~cm}^{-1}$ and $2009 \mathrm{~cm}^{-1}$ for the $v_{5}$ and $v_{4}$ modes, respectively. 20

There are more force constants in Eqn. (3.3) than there are frequencies to fit, and it is desirable to use the smallest possible number of parameters to fit the vibrational frequencies. First, we assume no coupling between the bonds ( $k^{\text {anisym }}, k^{5 y m, 1-3}=0$ ) and $k_{1}=k_{4}, k_{2}=k_{5}$ giving the potential

$$
2 V=k_{1}\left(\Delta r_{a}^{2}+\Delta r_{\theta}^{2}\right)+k_{2}\left(\Delta r_{b}^{2}+\Delta r_{d}^{2}\right)+k_{3} \Delta r_{c}^{2}
$$

The symmetric stretch frequencies can be fit using Eqn. (3.4), and the antisymmetric stretches obtained, $1112 \mathrm{~cm}^{-1}$ and $1971 \mathrm{~cm}^{-1}$, are cluse to the matrix values, but the resulting three force constants $\left(k_{1}=7.67\right.$ mdyne $/ \hat{A} ; k_{2}=10.44$ mdyne $/ \dot{A} ; k_{3}=3.93$ mdyne $\left./ \hat{A}\right)$ are so different as to be 
unrealistic. For example, ab initio calculations on $C_{6}$ predict the central C.C bond to be slightly shorter than the others, ${ }^{20}$ which is inconsistent with $k_{3}$ being the smallest force constant.

Alternatively, we can set all $k_{j}$ 's equal and assume coupling only between adjacent bonds $\left(k^{\text {syn.2 }}=0, k^{5 y m \cdot 3}=k^{\text {antisym }}\right)$ which gives the potential

$$
\begin{gathered}
V=\frac{1}{2}\left(\Delta r_{a}^{2}+\ldots+\Delta r_{e}^{2}\right)+k^{s y m 3}\left(\Delta r_{a} \Delta r_{b}+\Delta r_{d} \Delta r_{\theta}\right) \\
+k^{s y m 1}\left(\Delta r_{b} \Delta r_{c}+\Delta r_{c} \Delta r_{d}\right)
\end{gathered}
$$

Setting $k_{1}=7.28$ indyne $/ A, k^{5 y m, 3}=-2.65$ mdyne $/ A$ and $k^{5 y m, t}=1.23$ mdyne/A reproduces the observed symmetric stretches, but also gives $v_{4}=2052 \mathrm{~cm}^{-1}$, which is slightly high, and $v_{5}=$ $809 \mathrm{~cm}^{-1}$. While this model appears to.be more realistic than the previous model, it produces an anomolously low $v_{5}$ frequency. It is interesting to note that when typical carbon-carbon double

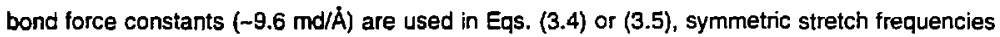
very similar to the ab initio results are obtained. ${ }^{18,20,41}$ As these are significantly higher than the experimental frequencies, the average bond order in $\mathrm{C}_{6}$ appears to be somewhat less than two.

Further calculations including more coupling constants were pertormed to reproduce the $1197 \mathrm{~cm}^{-1}$ antisymmetric stretch frequency along with our observed symmetric stretch frequencies. However, even with a great deal of coupling, a very smail center bond force constant was always required. Calcuiztions pertormed with the restriction that the center bond remain comparable to the other bonds consistently produced a $v_{5}$ frequency around $800 \mathrm{~cm}^{-1}$. This raises the possibility that the $v_{5}$ frequency, like the observed $v_{2}$ and $v_{3}$ frequencies, is much lower than predicted, and that the matrix assignment of the $1197 \mathrm{~cm}^{-1}$ line to the $v_{5}$ mode in $C_{6}$ is incorrect. We note that a weak, unassigned line at $814 \mathrm{~cm}^{-1}$ was seen by Weltner and McLeod in one of the first matrix isolation studies of carbon clusters, and that the $v_{5}$ absorption is predicted to be considerably less intense than the $v_{4}$ absorption. While our force constant analysis cannot be regarded as definitive, it suggests that it would be worthwhile to perform the 
same isotopic substution studies on the $1197 \mathrm{~cm}^{-1}$ and $814 \mathrm{~cm}^{-1}$ lines as were used to confim the assignment of the $1957 \mathrm{~cm}^{-1}$ line to $C_{6}$, or to study these transitions in a rotationally-resoived gas phase experiment.

\section{3) $\quad \mathrm{C}_{6}-\mathrm{C}_{6}$ geometry change}

The intensities of the peaks in the threshold spectrum are sensitive to the geometry change between the anion and neutral, and this change can be determined (approximately) by simulating the spectrum within the Franck-Condon approximation. The intensity of a transition between vibrational levels $v^{\prime \prime}$ and $v^{\prime}$ of the anion and neutral, respectively, is assumed proportional to the Franck-Condon factors (FCF):

$$
\left.\left|\left(v^{\prime} \leftarrow v^{\prime \prime}\right) \propto\right|\left\langle\chi_{\text {vib }}^{\prime}\right| \chi_{\text {vib }}^{\prime}\right)\left.\right|^{2}
$$

The vibrational wave functions, $\chi_{\text {vibr }}$ are taken to be a product of three harmonic oscillator wave functions corresponding to the three symmetric stretches. Since the anion symmetric stretch frequencies are unknown, the anion potentials are taken to be identical to the neutral potentials (hence with the same frequencies), only displaced along the normal coordinate. This displacement is adjusted until the relative intensities in the simulation match those in the spectrum. Figure (3.11) shows the Franck-Condon simulation of the threshold photodetachment spectrum along with the actual spectrum. The three normal mode displacements in the simulation are $\Delta \mathrm{Q}_{1}=0.033(\mathrm{amu})^{1 / 2} \mathrm{~A}, \Delta \mathrm{Q}_{2}=0.034(\mathrm{amu})^{1 / 2} \dot{\mathrm{A}}$, and $\Delta \mathrm{Q}_{3}=0.058(\mathrm{amu})^{1 / 2} \mathrm{~A}$. These displacements reproduce the four peak intensities and do not result in appreciable Iransitions to overtone or combination levels of $\mathrm{C}_{6}$.

In order to convert the normal mode displacement quantities to bond length changes, we first require a potential energy function in terms of bond length displacements and foice constants which reproduces the experimental frequencies. We use the potential of Eqn. (3.5) 
with the three force constants that gave the best fit to the symmetric stretch frequencies (listed previously).

To proceed further, we also require the sign of each normal mode displacement upon photodetachment. Unfortunately, the Franck-Condon factors depend only on the magnitudes of the displacements. There are eight possible combinations of signs in the three normal mode displacements, each yielding a different set of bond length changes. We can choose the most reasonable ccmbination by comparing ab initio calculations of the anion ${ }^{22,37}$ and neutral $18,20,41$ geometries with the bond length changes from each combination. Calculations on $\mathrm{C}_{6}$ predict the $\mathrm{C}-\mathrm{C}$ bond lengths in the range of $1.29 \mathrm{~A}$ which decrease slightly (by $0.02 \dot{A}$ ) from the outermost to the central bond. In contrast, larger bond length variations are predicted in $\mathrm{C}_{6}=$ Adamowicz 22 finds $r_{a}=1.292 A$ ([Outermost bond- see Figure (3.10)], $r_{b}=1.341 \dot{A}$, and $r_{c}=$ 1.290 $\dot{A}$, while Watts and Bantett ${ }^{37}$ find $r_{a}=1.258 \dot{A}, r_{b}=1.340 \dot{A}$, and $1.237 \dot{A}$. The most consistent result is that $r_{b}$ in the anion is significantly greater, about $0.050 \dot{A}$, than in the neutral, a physically reasonable result since the $2 \pi_{u}$ orbital from which photodetachment occurs is antibonding between the two carbon atoms. ${ }^{19}$

With our force constants, this lesult is reproduced only when all three normal coordinate displacements of the anion relative to the neutral to be positive. [Figure (3.10) shows what is meant by positive for each normal mode.] This yields $\Delta r_{a}=0.005 \dot{A}$ (anion-neutral), $\Delta r_{b}=0.057$ $A$, and $\Delta r_{c}=-0.057 \AA$. Varying the $\Delta Q_{n}$ in the Franck-Condon simuiation so that the simulated peak intensities have uncertainty falling within the experimental signal-to-noise changes the resultant bond length differences by about $\pm 0.005 \dot{A}$. Although geometry changes are in better overall agreement with Bartlett's calculations than those of f.damowicz, they do result from several significant approximations and should be treated with , wpropriate caution. It should also be noted that the calculated geometries on which we base the sign of our normal coordinate displacement were determined in the same calcuations that overestimated the $v_{2}$ and $v_{3}$ (and 
possibly $\left.v_{5}\right)$ frequencies. However. geometries obtained in ab initio calculations are generally more reliable than frequencies.

\section{4) Comparison to the $\mathrm{C}_{5}-$ threshold photodetachment spectrum}

The overall appearance of the $\mathrm{C}_{5}{ }^{-}$and $\mathrm{C}_{6}{ }^{-}$spectra is qualitatively different. In the $\mathrm{C}_{6}{ }^{-}$ spectrum, only transitions to excited symmetric stretch levels of $C_{6}$ are seen. These are all about one third the intensity of the origin transition. In contrast, in the $\mathrm{C}_{5}{ }^{-}$spectrum, transitions to several bend levels in the neutral are evident and are nearly as intense as the transition to the $C_{5}\left(v_{2}=1\right)$ symmetric stretch level. However, the transitions to excited vibrational states of $C_{5}$ are at least a factor of ten less intense than the origin. Transitions of this intensity would not be observable in the $\mathrm{C}_{6}{ }^{-}$spectrum because the signal-to-noise is such that the transitions would be obs :red.

$T$ e higher intensity symmetric stretch transitions in the $\mathrm{C}_{6}{ }^{-}$spectrum imply larger bond length changes upon photodetachment. This is corsistent with ab initio calculations of the $\mathrm{C}_{5}$ and $\mathrm{C}_{5}{ }^{-}$geometries. It therefore appears that the addition of an electron to the LUMO of $\mathrm{C}_{5}$, the $2 \pi_{u}$ orbital has less effect on the bond lengths than the addition of an electron to the (half-filled) $2 \pi_{\mathrm{u}}$ LUMO in $\mathrm{C}_{6}$.

\section{B. Partially discriminated and total photodetachment cross section spectra}

\section{1) Peak assignments}

Both the partially discriminated and total photodetachment cross section scans [Figures (3.4b) and (3.4c)] feature fairly intense manifolds of sharp peaks $-43 \mathrm{~cm}^{-1}$ to the red of the direct detachment thresholds, which correspond to the ZEKE peaks seen in Figure (3.4a). These peaks are presumably due to transitions to a $\left(\mathrm{C}_{6}{ }^{-}\right)^{\top}$ excited electronic state of the anion. Since these peaks so closely follow the direct detachment thresholds, they are assigned to the same vibrational transitions as the corresponding peaks in the threshold photodetachment spectrum. 
That is, the SA manifold marks the origin of the $\left(\mathrm{C}_{6}{ }^{-}\right)^{-} \leftarrow \mathrm{C}_{6}{ }^{-}$transition, and the SD, SG, and SI manifolds are assigned to the $3_{0}^{1}, 2_{0}^{1}$, and $I_{0}^{1}$ transitions, respectively.

This assignment explains the observation of the $3_{0}^{1}, 2_{0}^{1}$, and $1_{0}^{1}$ transitions. The upper states in these transitions, namely the $v_{1}=1, v_{2}=1$, and $v_{3}=1$ levels of the excited $\left(C_{6}-\right)^{*}$ state, can all decay to the $v=0$ level of neutral $C_{6}$ by vibrational autodetachment. However, the SA manifold lies to the red of the detachment continuum. If this manifold includes the band origin, then the $v=0$ level of the $\left.\left(\mathrm{C}_{6}\right)^{-}\right)^{\circ}$ excited state lies below the $v=0$ level of linear $\mathrm{C}_{6}$. Thus, autodetachment from the $\left(\mathrm{C}_{6}\right)^{-} v=0$ level should not be energetically possible. The SA manifold therefore requires further discussion. We also need to explain the sharp peaks $\left(b_{0}, e_{0}\right.$, etc.) that do not obviously correspond to peaks in the threshold photodetachment scan. In addition, there are congested clumps of peaks to the red of several of the strong transitions $a_{0}$. $d_{0}, g_{0}$, and $i_{0}$ in Figures (3.4b) and (3.4c) which need to be explained.

SA manifold. All of the peaks in the SA manifold appear at a lower photon energy than the minimum needed to remove an electron from ground state $C_{6}{ }^{-}$to form linear $C_{6}$. Two possible explanations for the appearance of this manifold in our photodetachment spectra are illustrated in Figure (3.12).

Figure (3.12a) shows that autodetachment can occur if peaks in the SA manifold are 'hot band' transitions originating from vibrationally excited anion states, thereby requiring less energy to excite the $\mathrm{C}_{6}{ }^{-}$to a state lying above the oetachment continuum. This hypothesis can be tested by varying the cluster source conditions to produce hotter ions, and observing how the intensities of the SA peaks vary relative to each other and to peaks in the higher-lying manifolds. The results [Figures (3.7a) and (3.7b)] show that the intensities of peaks $a_{0}$ and $d_{0}$ are insensitive to source conditions, but the peaks just to the red of $a_{0}$ and $d_{0}$ are noticeably larger in the 'hotter' scan [Figure (3.7b)]. Thus, the peaks to the red of peak $a_{0}$ in the photodetachment cross section arise from the situation in Figure (3.12a); they are sequence band transitions from vibrationally excited $\mathrm{C}_{6}{ }^{-}$to vibrationally excited levels of $\left(\mathrm{C}_{6}{ }^{-}\right)^{*}$ with sufficient energy to 
autodetach. However, the temperature studies suggest that peak $a_{0}$ at $297.019 \mathrm{~nm}$ is indeed the origin of the $\left(\mathrm{C}_{6}^{-}\right)^{-} \leftarrow \mathrm{C}_{6}^{-}$transition, and its presence still recuires explanation.

The most likely explanation for peak $a_{0}$ is that it resuits from a resonant two-photon transition where the first photon excites the ground anion electronic state to a bound excited anion state, and the second photon detaches the excited anion [Figure (3.12b)]. Resonant two photon detachment through an intermediate state was first observed by Lineberger for $\mathrm{C}_{2}-$ in 1972.42 This explanation is consisterit with several observations. In the first place. Figures (3.5a) and (3.5b) show that peak $a_{0}$ is virtually absent in the partially discriminated scan, indicating that the electron kinetic energy associated with this peak is considerably higher than for the peaks to the red. This is just what would occur if peak $a_{0}$ results from two-photon absorption, whereas the other peaks are from one-photon absorption just above the detachment threshold followed by autodetachment.

Furthermore, the results in Figure (3.8) indicate that the power dependence of peak $a_{0}$ differs from the other peaks in the SA manifold. The dependence of the intensity of peak $a_{0}$ on laser pulse energy is approximately linear, which at first glance suggests an unsaturated, onephoton process. However, the intensity of the adjacent peak, $a_{1}$, is proportional to the (pulse energy) ${ }^{1 / 2}$, which suggests a saturated one-photon transition. Although not conclusive, the $a_{0}$ power dependence is consistent with a two-photon process in which the first step (between the two bound anion levels in our hypothesis) is strongly saturated.

Finally, we consider the two-color studies shown in Figure (3.9). These were inspired by related work of Brauman and co-workers ${ }^{43}$ on organic anions. Figure (3.9) shows a low power ( $0.5 \mathrm{~mJ} / \mathrm{pu} / \mathrm{se})$ total cross section scan with and without a simultaneous, more intense pulse ( $2 \mathrm{~mJ} /$ pulse) at $532 \mathrm{~nm}$. The intensity of peak $a_{0}$ in the two-color scan is substantially increased, while that of peak $a_{1}$ is essentially unchanged. The $532 \mathrm{~nm}$ photon jannot detach ground state $C_{6}{ }^{-}$, but can detach electronically excited $\left(C_{6}\right)^{\circ}$. Our results indicate that the $\left(C_{6}\right)^{\circ}$ state for peak $a_{0}$ is sutficiently long-lived so that it can be photodetached by a second photon, 
whereas the upper state for peak $a_{1}$ autodetaches too rapidly for the second photon to make any difference. This further supports the idea that peak $a_{0}$ is a transition to a level of $\left(C_{6}-\right)^{*}$ which is bound with respect to autodetachment. while peak $a_{1}$ is a transition to $a$ level above the detachment threshold.

All of this evidence shows that peak $a_{0}$ indeed is the vibrational origin of the $\left(\mathrm{C}_{6}^{-}\right)^{*} \leftarrow$ $\mathrm{C}_{6}{ }^{-}$transition. Based on a comparison with the threshold photodetachment scan, the $v=0$ level of the excited anion state lies $43 \mathrm{~cm}^{-1}$ below the $v=0$ level of neutral $C_{6}$. This explains why the intensity of peak $a_{0}$ in the total cross section scan [Figure (3.4c)] is lower relative to the $10^{9}, 2_{0}{ }^{1}$. and $30^{\prime}$ transitions than in the threshold photodetachment spectrum [Figure (3.4a)], where the origin transition is dominant. If, in the total cross section scan, peak $a_{0}$ is the only major peak that results from two-photon absorption, it is not surprising that its intensity is anomalously low.

Other manifolds. The assignment of the less intense but shap teatures in the total cross section scan is not so obvious since there are no corresponding peaks in the neutral spectrum. While peak $f_{0}$ appears to be the $3_{0}^{2}$ transition, the remaining peaks are most likely due to to transitions to bend and antisymmetric stretch levels in the upper anion state; such transitions are allowed so long as the vibrational quantum number of the active mode changes by an even number.

Although no calculations for vibrational frequencies have been periormed for the $\left(C_{6}{ }^{-}\right)^{*}$ state, its symmetric stretch frequencies are essentially identicai to those of neutral $C_{6}$. Assuming this holds for the non-totally symmetric vibrations as well, the less intense autodetaciment peaks can be tentatively assigned by comparison with the corresponding frequencies from ab initio calculations performed on finear neutral $C_{6}{ }^{20}$ The assignments and comparison with ab initio values are shown in Table (3.2). For example, peak $\mathrm{b}_{0}, 186 \mathrm{~cm}^{-1}$ to the blue of the origin, is assigned to the $9_{0}^{2}$ transition; the $v_{9}\left(\pi_{u}\right)$ mode is predicted to be the lowest frequency bend mode $\left(216 \mathrm{~cm}^{-1}\right.$ for two quanta). ${ }^{20}$ Peak $\mathrm{c}_{0}, 390 \mathrm{~cm}^{-i}$ to the blue of the origin, is assigned to the $7_{0}^{2}$ transition, in accordance with the calculated energy of $446 \mathrm{~cm}^{-1}$ for 
the $v_{7}=2$ level; the $v_{7}$ mode, a $\pi_{9}$ bend, is predicted to be the nert-lowest frequency bending mode. Similarly, peak $e_{0}$ at $626 \mathrm{~cm}^{-1}$ to the blue of the origin is assigned to the $8_{0}^{2}$ transition and peak $h_{0}$ found at $1674 \mathrm{~cm}^{-1}$ to the blue of the origin is assigned to the $5_{0}^{2}$ transition. These latter two assignments are very tentalive, since the deviation from the $a b$ initio frequencies is significant, although the assigned $v_{5}$ frequency is close to the $v_{5}$ frequency determined in the one-dimensional calculation on $\mathrm{C}_{6}$ using Eqn. (3.5). The remaining shap autodetachment peaks all appear at energies which are sums of the preceding bend and stretch frequencies, so they are assigned to combination transitions.

Sequence Bands. Finally, we consider the sequence bands which are most evident to the red of peaks $a_{0}$ and $d_{0}$; see Figures (3.5) and (3.6). The temperature studies [Figure (3.7)] show that most of these are transitions from vibrationally excited $C_{6}{ }^{-}$in its ${ }^{2} \Pi_{u}$ state to excited vibrational levels of the $\left(\mathrm{C}_{6}^{-}\right)^{*}$ state. The assignment of th:ese features is not straightforward; several of the peaks are only partially resolved, particuiarly in Figure (3.5b). However, there is a pattem to the peak spacings, with intervals of $18 \mathrm{~cm}^{-1}, 23 \mathrm{~cm}^{-1}$, and $29 \mathrm{~cm}^{-1}$ appearing several times. In addition, the assignment is aided by comparing differences in peak intensities in the partially discriminated and total cross section scans, which are sensitive to the energy of the ejected electron, and the enhancement of peak intensities in the two-photon, two-color scan [Figure (3.9)], which is sensitive to the lifetime of the upper state with respect to autodetachment. Sets of peaks for which the intensities vary in the same way are most likely due to a common (or similar) upper state. Also, any two peaks split by $1.7 \mathrm{~cm}^{-1}$ are assigned to the same vibronic transition; as discussed above, this splitting is probably due to rotational contours in which individual rotational transitions are not resolved.

Peaks $a_{0}, a_{2}$, and $a_{3}$ appear in the total cross section scan but are missing in the partially discriminated scan. Peaks $a_{0}$ and $a_{3}$ are both doublets split by $1.7 \mathrm{~cm}^{-1}$. They are separated by $29 \mathrm{~cm}^{-1}$, which is the previously determined spin-orbit interval in the ${ }^{2} \Pi_{u}$ ground state of $\mathrm{C}_{6}-$, and are both significantly enhanced in the two-color, two-photon experiment. Thus, 
we assign $a_{0}$ to the $0_{0}^{0}$ transition originating from the ${ }^{2} \Pi_{3 / 2}$ leve! of $C_{6}{ }^{-}$, and peak $a_{3}$ to the same transition originating from the spin-orbit excited $2 \Pi_{1 / 2}$ level. This assignment implies that the spin-orbit splitting in the $\left(\mathrm{C}_{6}^{-}\right)^{*}$ is negligible, a point which will be addressed later, and that the upper state of peak $a_{3}$, like thet of peak $a_{0}$, is bound with respect to autodetachment. Peak $a_{2}$ is discussed below.

Peak $a_{1}, 18 \mathrm{~cm}^{-1}$ to the red of peak $a_{0}$, is the second most intense feature in Figure (3.5b) and the most intense feature ir: Figure (3.5a). The significant intensity of this peak and its strong temperature dependence suggest it originates from the lowest excited vibrational level of $\mathrm{C}_{6}-$, which, based on ab initio calculations on neutral $C_{6}$, should be the $v_{9}=1$ bending level. We therefore assign peak $a_{1}$ to the $9_{1}^{1}$ sequence band. Our previous assignment of peak $b_{0}$ to the $9_{0}^{2}$ transition, yields $93 \mathrm{~cm}^{-1}$ for the $v_{9}$ frequency in the $\left(C_{6}{ }^{-}\right)^{*}$ state, so the assignment of peak $a_{1}$ yields a $v_{9}$ frequency of $111 \mathrm{~cm}^{-1}$ for the $C_{5}-$ ground state. With this assignment, the broad $a_{4}$ peak in Figure (3.5a), which is approximately $16 \mathrm{~cm}^{-1}$ to the red of the $a_{1}$ doublet, is assigned to the $9_{2}^{2}$ transition. The assignment of these peaks to transitions involving the $v_{9}$ mocie is consistent with the observation of peak $a_{1}$ with high intensity in the partially discriminated scans [Figures (3.4b), (3.5a)]. The decay of the $v_{9}=1$ level of $\left(C_{6}{ }^{-}\right)^{*}$ by vibrational autodetachment will result in a photoelectron with only $50 \mathrm{~cm}^{-1}$ of kinetic energy [obtained by subtracting $43 \mathrm{~cm}^{-1}$, the amount by which the vibrationless $\left(\mathrm{C}_{6}^{-}\right)^{\circ}$ state is bound, from $93 \mathrm{~cm}^{-1}$, the $v_{9}=1$ vibrational energy in the $\left(C_{6}{ }^{-}\right)^{*}$ state], and this will be collected efficiently in the partially discriminated mode of operation.

Peak $a_{6}$ is $29 \mathrm{~cm}^{-1}$ to the red of peak $a_{1}$, the same interval between peaks $a_{3}$ and $a_{0}$, so we assign it to the $9_{1}^{1}$ transition from the spin-orbit excited ${ }^{2} \Pi_{1 / 2}$ level of $\mathrm{C}_{6}{ }^{-}$. The small peak $a_{8}, 16 \mathrm{~cm}^{-1}$ to the red of peak $a_{6}$, is then assigned to the $9_{2}^{2}$ transition from the same spin-ortit level. The relative intensities of peak $a_{6}$ and $a_{1}$ are similar in Figures (3.5a) and (3.5b), lending further support to our assignment. 
Peak $a_{2}$ is $25 \mathrm{~cm}^{-1}$ to the red of peak $a_{0}$, and peak $a_{5}$ is $23 \mathrm{~cm}^{-1}$ to the red of peak $a_{1}$. It is reasonable to assign peak $a_{2}$ to the $7_{1}^{1}$ sequence band transition, since the $v_{7}$ bending mode should be the second lowest frequency vibration in $C_{6}-$. Our previous assignment of peak $c_{0}$ to the $7_{0}^{2}$ transition gives $v_{7}=195 \mathrm{~cm}^{-1}$ in the $\left(C_{6}^{-}\right)^{*}$ state, so assigning peak $a_{2}$ to the $7_{1}^{1}$ transition yields $v_{7}=220 \mathrm{~cm}^{-1}$ in the anion ground state. Peak $a_{5}$ is then the 7,$9 ;$ combination transition. We assign peak $a_{7}, 15 \mathrm{~cm}^{-1}$ to the red of peak $a_{5}$, to the $7 \frac{1}{1} 9_{2}^{2}$ transition.

Note that peak $a_{2}$ is prominent in the total cross section scan but not in the partially discriminated scan, while peaks $a_{5}$ and $a_{7}$ are clearly visible in the partially ciscriminated scan. This indicates that the ejected photoelectrons are less energetic for peaks $a_{5}$ and $a_{7}$. Vibrational autodetachment from the $v_{7}=1$ level of $\left(C_{6}^{-}\right)^{*}$ (the presumed upper level for peak $a_{2}$ ) to the $C_{6}$ ground state results in a photoelectron with $152 \mathrm{~cm}^{-1}$ of kinetic energy. On the other hand, both the $\left(v_{7}=1, v_{9}=1\right)$ and $\left(v_{7}=1, v_{9}=2\right)$ levels of the $\left(C_{6}^{-}\right)^{-}$can autodetach via a $\Delta v_{9}=-1$ transition, yielding $50 \mathrm{~cm}^{-1}$ electrons in both cases. Hence, the observed intensities of the three peaks in Fiçures (3.5a) and (3.5b) is qualitatively consistent with our assignment.

Table (3.3) gives a summary of all of the sequence band assignments. Several of these assignments, particularly those involving the $v_{7}$ mode, must be regarded as somewhat tentative. Also, we have not considered complications from Renner-Teller activity in the excited bending modes for the anion ${ }^{2} \Pi_{u}$ ground state. A possible contribution from this effect is the additional feature $a_{1}{ }^{\prime}$ in Figure (3.5b). The $a_{1}{ }^{\prime}-a_{1}$ feature is noticeably broader than the $a_{0}$ doublet and in fact appears to be two superimposed doublets separated by $2 \mathrm{~cm}^{-1}$. This could directly reflect the Renner-Teller splitting in the $v_{9}$ mode of the anion.

\section{2) Autodetachment mechanism and autodetaching state lifetimes.}

The mechanisms for autodetachment of negative ions and the analogous process, autoionization of neutrals, have been extensively discussed in the literature.44,45,46,47 For our $\left(\mathrm{C}_{6}^{-}\right)^{\circ}$ state, where the $v=0$ level is bound but all vibrationally excited levels lie above the 
detachment threshold, vibrational autodetachment is the most likely mechanism. In this case, autodetachment occurs by a breakdown of the Born-Oppenheimer approximation. The electronic wave functions for the excited anion state and neutral are coupled via the nuclear vibrational kinetic energy operator $T_{v i b r}$ and autodetachment occurs when one (or more) quanta of vibrational energy in the anion is converted to electronic energy, resulting in the ejection of an electron.

More specifically, the vibrational autodetachment rate from the initial state with $v_{k}=n$ is given by ${ }^{45}$

$$
\text { rate } \propto \frac{2 \pi}{\hbar}\left|\left\langle\Psi_{f}\left|\hat{T}_{v i h}\right| \Psi_{i}\right\rangle\right|^{2} \rho
$$

where $\Psi_{i}=\Psi_{e l, i} \chi_{v i b, j}$ and $\Psi_{f}=\Psi_{e l, j} \chi_{v i b, f}$ are the vibronic wave functions for the excited anion and (neutral + electron), respectively, and $\rho$ is the density of states for the (neutral + electron) continuum. The matrix element in Eqn. (3.7) is given by

$$
\left\langle\Psi_{f}\left|\hat{T}_{v i b}\right| \Psi_{i}\right\rangle=\frac{-\hbar}{\mu}\left\langle\chi_{v i b, s}\left\langle\Psi_{e l, s} \mid \frac{d}{d Q_{k}} \Psi_{e l, i}\right\rangle \frac{d}{d Q_{k}} \chi_{v t h, i}\right\rangle .
$$

If the inner integral over electronic coordinates is assumed constant, and $\chi_{v i b, l}$ and $\chi_{v i b, f}$ are taken to be harmonic oscillator wave functicus, then only $\Delta v_{k}=-1$ transitions are allowed. This propensity rule was used in the previous section in determining the electron kinetic energies from autodetachment out of excited vibrational levels of the $\left(\mathrm{C}_{6}^{-}\right)^{\prime}$ state, although for several of these states, $\Delta v_{k}=-1$ transitions are the only possible transitions.

The lifetimes of autodetaching states are best determined by measuring spectra! linewidths. Unfontunately, we cannot resolve individual rovibronic transitions in these spectra. We can estimate limits on the lifetimes, however, based on a few observations. 
We first consider the transitions to excited symmetric stretch levels of the $\left(\mathrm{C}_{6}{ }^{-}\right)^{*}$ state. Peak $d_{0}$, the $3_{0}^{\prime}$ transition, shows the characteristic $1.7 \mathrm{~cm}^{-1}$ splitting attributed to rotational contours. This splitting is not resolved for peaks $g_{0}\left(2_{0}^{1}\right)$ and $i_{0}\left(1_{0}^{1}\right)$. If this lack of structure is due to lifetime broadening, then the autodetachment lifetimes of the $v_{1}=1$ and $v_{2}=1$ leveis are less thar, 1 ps, while that of the $v_{3}=1$ level is longer (actually much longer, as shown below). However, in the absence of full rotational resolution, we cannot rule out spectral congestion as the source of the broadening of the $2_{0}{ }^{1}$ and $1_{0}{ }^{1}$ transitions.

We obtain another, more iriteresting measure of autodetachment lifetimes from the twophoton, two-color scans [see Figure (3.9) and Table (3.4)]. If the intensity of a peak is enhanced in those scans, then the state accessed by the ultraviolet photon is either bound and has a fluorescence lifetime comparable to or greater than the laser pulse width (peaks $a_{0}, a_{3}$ ), or it is quasibound with its autodetachment lifetime comparable to or greater than its fluorescence lifetime as well as the laser pulse width (predissociation should not be energetically allowed). In the latter case, the autodetachment lifetime must be in the nanosecand range. We find that peaks $d_{0}, d_{2}, a_{2}$ and $a_{7}$ are noticeably enhanced; the first two are the two spin-ortit components of the $3_{0}{ }^{1}$ transition, and the second two are sequence band transitions in which the upper stafe can autodetach only by a $\Delta v_{7}=-1$ transition. Hence, the lifetimes for autodetachment through the $v_{7}$ and $v_{3}$ modes are at least several nanoseconds. On the other hand, the peaks in which the upper state can autodetach via a $\Delta v_{9}=-1$ transition $\left(a_{1}, a_{6}\right)$ show no enhancement, but the $1.7 \mathrm{~cm}^{-1}$ splitting is still resolvable. So long as the fluorescence lifetime is similar for the $v_{3}=1, v_{7}=1$, and $v_{9}=1$ levels of the $\left(C_{6}{ }^{-}\right)^{-}$state. this result means that the autodetachment lifetime of a state which can autodetach by a $\Delta u_{9}=-1$ transition is greater than 2 ps, but is significantly shorter than the autodetachment lifetime of a state that must autodetach by a $\Delta v_{7}=-1$ or $\Delta v_{3}=-1$ transition.

This mode-specific effect is striking and deserves further discussion. The density of states factor $\rho$ in Eqn. (3.7), which is proportional to the (electron kinetic energy) ${ }^{1 / 2}$, is smaller 
Ior a $\Delta v_{9}=-1$ transition than for a $\Delta u_{7}=-1$ or $\Delta v_{3}=-1$ transition. Thus, $\left\langle\Psi_{j} \mid T_{v i b} j \Psi_{j}\right\rangle$ mi mst be substantially larger for a $\Delta v_{9}=-1$ transition. This will be the case if the inner integral in Eqn. (3.8) over the electron coordinates, $\left.\left\langle\psi_{e l, f} f d / \psi_{e l, i}\right) / d \mathrm{Q}_{k}\right\rangle$, is larger for the $v_{9}$ mode than for the other two modes, which, in turn, requires that the derivative of the $\left(\mathrm{C}_{6}-\right)^{*}$ electronic wave function with respect to $O_{9}$ is large. While the nature of $\psi_{e l i}$ is uncertain (see next section), the $v_{9}$ mode is a low-frequency, large amplitude bending mode. One might therefore expect the electronic wave function to be quite different at the classical turning points for the $v_{9}=1$ level than at the equilibrium geometry, while for a higher frequency, lower amplitude mode, this effect would not be so pronounced. A more quantitative treatment along the lines developed by Simons ${ }^{48}$ would be extremely useful in understanding the autodetachment phenomena observed here.

\section{3) Nature of the excited anion state.}

The molecular orbital configurations of the $\mathrm{C}_{6}-$ and $\mathrm{C}_{6}$ ground states are

$$
\begin{array}{ll}
C_{6}-\left({ }^{2} \Pi_{u}\right): & \ldots\left(1 \pi_{u}\right)^{4}\left(6 \sigma_{u}\right)^{2}\left(7 \sigma_{g}\right)^{2}\left(1 \pi_{g}\right)^{4}\left(2 \pi_{u}\right)^{3} \\
C_{6}\left({ }^{3} \Sigma_{g}-\right): & \ldots\left(1 \pi_{u}\right)^{4}\left(6 \sigma_{u}\right)^{2}\left(7 \sigma_{g}\right)^{2}\left(1 \pi_{g}\right)^{4}\left(2 \pi_{u}\right)^{2}
\end{array}
$$

The $\left(\mathrm{C}_{6}{ }^{-}\right)^{*}$ excited electronic state can resuit from excitation from one of the lower-lying orbitals to the partially-filled $2 \pi_{u}$ orbital, or by promotion of a $2 \pi_{u}$ electron to a higher-lying, empty orbital. The correspondence between vibrational features in the $\mathrm{C}_{6}{ }^{-}$threshold photodetachment and autodetachment spectra indicates that $\left(\mathrm{C}_{6}^{-}\right)^{\circ}$ and neutral $\mathrm{C}_{6}$ have similar nuclear geometries. This is consistent with the second type of excited state, with the added proviso that the $2 \pi_{u}$ electron is sromoted to a non-bonding orbital.

The nature of this orbital is of considerable interest. Autodetachment has previously been observed from two types of excited electronic states in molecular negative ions: valence states, in which the highest occupied molecular orbital is a 'conventional' molecular orbital, and 
electrostatically bound states, in which the outermost electron is in a very diffuse orbital and is bound to the neutral core via long-range electrostatic attraction. An example of the former is $\mathrm{C}_{2} ;{ }^{26}$ while examples of the latter include dipole-bound states, such as in $\mathrm{CH}_{2} \mathrm{CHO}^{-49}$ and $\mathrm{CH}_{2} \mathrm{CN}^{-}, 50$ and the 'image charge-bound' states proposed to explain autodetachment in $\mathrm{Au}_{6}{ }^{-5} .51$

The $\left(\mathrm{C}_{6}^{-}\right)^{*}$ state does not appear to be a clear-cut example of either type of excited state. The similarity between the $\left(\mathrm{C}_{6}\right)^{\circ}$ and $\mathrm{C}_{6}$ nuclear geometries is what would be expected if $\left(\mathrm{C}_{6}^{-}\right)^{*}$ were electrostatically bound, consisting of an electron weakly interacting with the neutral $\mathrm{C}_{6}$ core. The apparent absence of spin-orit splitting in the $\left.\left(\mathrm{C}_{6}\right)^{-}\right)^{-}$state is consistent with such a picture. In addition, the small binding energy $\left(43 \mathrm{~cm}^{-1}\right)$ is characteristic of such a state. While $\left(\mathrm{C}_{6}\right)^{-}$"obviously cannot be a dipole-bound state, an 'image charge-bound' state (or, perhaps more accurately, a 'polarization-bound' state) cannot be ruled out. These states have not been nearly as thoroughly investigated as dipole-bound states, 52 but such a state might result if the polarizability of the $\pi$-electron network in the $\mathrm{C}_{6}$ core were sufficiently high. However, we do not observe autodetachment near the detachment threshold for $\mathrm{C}_{4}{ }^{-}$or $\mathrm{C}_{B^{-}}$. If $\left(\mathrm{C}_{6}{ }^{-}\right)^{*}$ is an electrostatically bound state, it appears to be a special case among carbon cluster anions.

The $\left(\mathrm{C}_{6}{ }^{*}\right)^{*}$ state could also resuit from excitation to an excited valence orbital. While bound valence-excited states for anions are rare, $\mathrm{C}_{6}{ }^{*}$ is an open-shelled molecule with a high electron binding energy and has several low-iying, unfilled $\pi$ orbitals, so the existence of such a state would not be surprising. This is supported in the work by Adamowicz, ${ }^{22}$ who calculated vertical excitation energies to eight bound excited anion states of $\mathrm{C}_{6}{ }^{-}$which involve only valence orbitals. In particular, an optically accessible ${ }^{2} \Pi_{g}$ state is predicted to lie just below the detachment threshold; this state results from excitation of a $2 \pi_{u}$ electron to a low-lying $\pi_{g}$ " orbilal.

If this state is actually the observed $\left(C_{6}{ }^{-}\right)^{*}$ state, then the $\pi_{9}{ }^{*}$ orbitai must be completely non-bonding, so that the excited anion state geometry is similar to that of the neutral. In addition, we observe the same spin-ortit splitting $\left(29 \mathrm{~cm}^{-1}\right)$ in the autodetachment spectrum as 
in the threshold photodetachment spectrum, indicating negligible spin-orbit interaction in the $\left(C_{6}^{-}\right)^{*}$ state (there is none in the $C_{6}{ }^{3} \Sigma_{g}{ }^{-}$state). Thus, if the $\left(C_{6}^{-}\right)^{*}$ state is indeed $a^{2} \Pi_{g}$ state, it must be very good example of Hund's case (b) coupling. Whether these constraints on the $\pi_{\mathrm{g}}{ }^{*}$ orbital eliminate it from consideration remain to be seen. Of course, the upper state could also be a $\Sigma_{g}$ state resulting from excitation of a $2 \pi_{\mu}$ electron to a $\sigma_{g}$ orbital; this would be consistent with the observed spin-orbit structure. No such states have been predicted near the detachment threshold, however.

The above discussion shows that the classification of the $\left(\mathrm{C}_{6}^{-}\right)^{\circ}$ state as an electrostatically bound or valence state is difficult. Both labels have their attractions and their pitfalls. In our view, the evidence points more towards a valence state at this point. More isight should result from rotationally-resolved resonant two-photon detachment experimentr via the bound $\left(\mathrm{C}_{6}\right)^{*}$ levels. In addition, ab initio studies of the geometries, and not just the vertical excitation energies, of the $C_{6}{ }^{-}$excited states would be extremely useful.

\section{v. Conclusion}

We have performed two types of photodetachment spectroscopy on $\mathrm{C}_{6}{ }^{*}$ : threshold photodetachment (ZEKE) spectroscopy, which maps out transitions between $\mathrm{C}_{6}{ }^{-}$and $\mathrm{C}_{6}$, and autodetachment spectroscopy, which reveals an excited state of $\mathrm{C}_{6}^{-} 43 \mathrm{~cm}^{-1}$ below the detachment threshold.

The vibrational and spin-orbit structure in the threshold photodetachment spectrum is consistent with a transition between linear $\mathrm{C}_{6}{ }^{-}$and $\mathrm{C}_{6}$. The spectrum yields the three symmetric stretch frequencies of $C_{6}$ as well as the spin-orbit splitting in the $C_{6} \cdot{ }^{-}$ground ${ }^{2} \Pi_{4}$ state. Two of our three frequencies are noticeably lower than previous $a b$ initio harmonic results. From these frequencies and the peak intensitles, we have used a simple force field model to derive force constants and bond displacements between the anion and neutral. In order to match the 
experimental frequencies, the force constants between adjacent carbon atoms had to be dropped significantly from typical carbon-carbon double bond force constants.

The autodetachment results show that the $\left(\mathrm{C}_{6}{ }^{-}\right)^{*}$ state is very similar to the neutral linear $C_{6}$ state. We observe transitions to the origin and excited symmetric stretch levels of the $\left(C_{6}\right)^{*}$ state which are all shifted $43 \mathrm{~cm}^{-1}$ to the red of corresponding features of the threshold photodetachment spectrum. Since the $v=0$ level of the $\left(\mathrm{C}_{6}{ }^{-}\right)^{*}$ state is bound with respect to the linear neutral, the electron signal we observe from this state results from a two-photon process. We also observe weaker transitions to excited bending and antisymmetric stretch levels of the $\left(\mathrm{C}_{6}{ }^{-}\right)^{*}$ state, as well as sequence bands originating from vibrationally excited levels of the $\mathrm{C}_{6}{ }^{-}$ ground state. Two-color, two-photon studies indicate differing autodetachment lifetimes for the various vibrational levels of the $\left(\mathrm{C}_{6}^{-}\right)^{*}$ state; autodetachment by a $\Delta v_{g}=-1$ transition ( $\pi_{4}$ bend) is noticeably faster than $\Delta v_{3}=-1$ (symmetric stretch) or $\Delta v_{7}=-1$ ( $\pi_{9}$ bend) transitions. We lean towards assigning the $\left(\mathrm{C}_{6}\right)^{*}$ state as a valence-excited state rather than an electrostatically bound state. $A b$ initio calculations predict a valence-excited ${ }^{2} \Pi_{g}$ state near the detachment threshold, but the $\left(\mathrm{C}_{6}\right)^{*}$ state does exhibit several properties one would expect in an electrostatically bound state.

Finally, we point out that while this experinent shows no evidence for a cyclic $C_{6}$ state close in energy to the linear $C_{6}$ state, the existence of such a state cannot be ruled ois. Any transitions from a linear anion to cyclic neutral will be spread out over a large energy range due to the substantial geometry change and therefore would be difficult to observe in this experiment. Although our earlier $\mathrm{C}_{6}-$ photoelectron spectrum showed a low energy 'tail' which may have come from cyclic $C_{6}$, which is predicted to lie about $1 \mathrm{eV}$ above the linear anion geometry, no such tail was seen in the threshold photodetachment spectrum. This may indicate less cyclic $\mathrm{C}_{6}{ }^{-}$under the beam source operating conditions used here. In any case, if there is a cyclic $C_{6}$ state lower in energy than linear $C_{6}$, then the levels of the excited $\left(C_{6}{ }^{-}\right)^{*}$ state which are bound with respect to linear $C_{6}$ should be able to autodetach to cyclic $C_{6}$. It may be possible 
to determine if this occurring by measuring the $\mathrm{C}_{6}{ }^{-}$photoelectron spectrum at the photon energy corresponding to peak $a_{0}$. This experiment will be carried out shonly in our laboratory.

\section{Acknowledgements}

This research is supported by the National Science Foundation under Grant No. CHE8857636. 
1J. B. Edwards, Combustion; Formation and Emission of Trace Species (Ann Arbor Science, Ann Arbor, 1974); J. R. Heath, S. C. O'Brien, R. F. Cur, H. W. Kroto, and R. E. Smalley, Comm. Cond. Mat. Phys. 13, 119 (1987); H. W. Kroto and K. McKay, Nature 331, 328 (1988).

${ }^{2}$ A. E. Douglas, Nature 269, 130 (1979); R. F. Knake, Nature 269, 132 (1979); H. W. Kroto, J. R. Heath, S. C. O'Brien, R. F. Curl, and R. E. Smailey, Astrophys. J. 314. 352 (1987); K. H. Hinkle, J. j. Keady, and P. F. Bemath, Science 241, 1319 (1988).

3W. Weltner, Jr., and R. J. Van Zee, Chem. Rev. 89, 1713 (1987).

${ }^{4 K}$. P. Huber and G. Herzberg, Molecular Spectra and Molecular Structure IV; Constants of Diatomic Molecules (Van Nostrand Reinhold, New York, 1979) Appendix IX and references therein.

5Representative references include: W. Huggins, Proc. R. Soc. London 33, 1 (1882); D. A. Ranisay, Adv. Spectrosc. 1, 1 (1959); G. Herzberg, Astrophys. 3. 96, 314 (1949); L. Gausset, G. Herzberg, A. Lagerquist, and B. Rosen, Discuss. Faraday Soc. 35. 113 (1965); K. Matsumura, H. Kanamori, K. Kawaguchi, and E. Hirota, J. Chem. Phys. 89, 3491 (1988); E. A. Rohlfing, J. Chem. Phys. 91, 4531 (1989).

${ }^{6}$ W. Weltner, Jr., P. N. Walsh, and C. L. Angell, J. Chem. Phys. 40. 1299 (1964); J. Chem. Phys. 40, 1305 (1964); J. Chem. Phys. 45, 3096 (1966).

7K. A. Thompson, R. L. DeKock, and W. Weitner, Jr., J. Am. Chem. Soc. 93, 4688 (1971).

8W. R. M. Graham, K. I. Dismuke, and W. Weltner, Jr., Astrophys. J. 204, 301 (1976); W.

Krätschmer, N. Sor z, and D. R. Hutfman, Surf. Sci. 156, 814 (1985); M. Vala, T. M.

Chandrasekhar, J. Szczepanski, R. J. Van Zee, and W. Weitner, Jr., J. Chem. Fhys. 90, 595 (1989).

9S. Yang, K. J. Taylor, M. J. Craycraft, J. Conceicao, C. L. Pettiette, O. Cheshnovsk, and A. E. Smalley, Chem. Phys. Lett. 144, 431 (1988). 
${ }^{10} \mathrm{C}_{3}$ : C. A. Schmuttenmaer, R. C. Cohen, N. Pugliano, J. R. Heath et al, Scien:ce 249, 897 (1990); C 4 : J. R. Heath and R. J. Saykally, J. Chem. Phys. 94, 3271 (1990); C 5 : J. R. Heath. A. L. Cooksy, M. H. W. Grubele, C. A. Schmuttenmaer, and R. J. Saykally, Science 244, 564 (1989); C 6 : H. J. Huang, A. Van Orden, K. Tanaka, E. W. Kuo, J. R. Heath, and R. J. Saykally, Mol. Phys. 79, 769 (1993); C.: J. R. Heath, A. Van Orden, E. Kuo, and F. J. Saykally, Chem. Phys Lett. 182, 17 (1991); J. R. Heath, and R. J. Saykally, J. Chem. Phys. 94,1724 (1991); J. R. Heath, R. a. Sheeks, A. L. Cooksy, and R. J. Saykally, Science 249, 895 (1990); Cg: J. R. Heath, and F. J. Saykally, J. Chem. Phys. 93, 8392 (1990); $C_{13}:$ T. F. Giesen, A. Van Orden, H. J. Hwang, R. S. Fellers, R. A. Provençal, and R. J. Saykally, Science 265, 756 (1994).

${ }^{11}$ H. Sasada, T. Amano, C. Jarman, and P. F. Bemath, J. Chem. Phys. 94, 2401 (1991); N. Moazzen-Ahmati, A. R. McKellar, and T. Amano, J. Chem. Phys. 91, 2140 (1989).

12P. F. Bernath, K. H. Hinkle, and J. J. Keady, Science 244, 562 (1989).

13K. M. Ervin and W. C. Lineberger, J. Phys. Chem. 95, 1167 (1991).

14M. Pollak, M. Gilles, and W. C. Lineberger (private communication).

15D. W. Amold, S. E. Bradforth, T. N. Kitsopoulos, and D. M. Neumark, J. Chem. Phys. 95, 8753 (1991).

16K. S. Pitzer and E. Clementi, J. Am. Chem. Soc. 81, 4477 (1959).

17T. N. Kitsopoulos, C. J. Chick, Y. Zhao, and D. M. Neumark, J. Chem. Phys. 95,5479 (1991).

18K. Raghavachari, R. A. Whiteside, and J. A. Pople, J. Chem. Phys. 85, 6623 (1986).

19V. Parasuk and J. Almölf, J. Chem. Phys. 91,1137 (1989).

20J. M. L. Martin, J. P. François, and R. Gijbels, J. Chem. Phys. 93, 8850 (1990); J. Comput. Chem. 12, 52 (1991). The listed calculations were performed on the MP2/6-31G" level. 
${ }^{21}$ K. Raghavachari, Z. Phys. D 12, 61 (1989).

22L. Adamowicz, Chem. Phys. Lett. 182, 45 (1991).

23M. Vala, T. M. Chandrasekhar, J. Szczepanski, and R. Pellow, High Tem. Sci. 27, 19 (1990).

${ }^{24}$ R. J. Van Zee, R. F. Ferrante, K. J. Zeringue, W. Weltner, Jr., and D. W. Ewing, J. Chem. Phys. 88, 3465 (1988).

${ }^{25} \mathrm{H}$. Heldman, D. Kella, E. Malkin, E. Miklazky, Z. Vager, J. Zajfman, and R. Naaman, J. Chem. Soc. Faraday Trans. 86, 2469 (1990); M. Algranati, H. Heldman, D. Kella, E. Malkin, E. Miklazky, R. Naaman, Z. Vager, and J. Zajfman, Israel J. Chem. 30, 79 (1990).

26P. L. Jones, R. D. Mead, B. E. Kohler, S. D. Rosner, and W. C. Lineberger, J. Chem. Phys. 73, 4419 (1980); U. Hefter, R. D. Mead, P. A. Schulz, and W. C. Lineberger, Phys. Rev. A 28, $1429(1983)$.

27P. A. Schulz, R. D. Mead, P. L. Jones, and W. C. Lineberger, J Chem. Phys. 77, 1153 (1982).

${ }^{28}$ G. F. Ganteior, D. M. Cox, and A. Kaldor, J. Chem. Phys. 94, 854 (1991).

29,G. F. Gantefor, D. M. Cox, and A. Kaldor, J. Chem. Phys. 96, 4102 (1992).

30T. N. Kitsopoulos, I. M. Waller, J. G. Loeser, and D. M. Neumark, Chem. Phys. Lett. 159, 300 (1989); T. N. Kitsopoulos, C. J. Chick, Y. Zhao, and D. M. Neumark. J. Chem. Phys. 95, 1441 (1991).

31T. G. Dietz, M. A. Duncan, D. E. Powers, and R. Z. Smalley, J. Chem. Phys. 74, 6511 (1981).

32J. M. B. Bakker, J. Phys. E 6, 785 (1973); J. Phys. E 7, 364 (1974). 
${ }^{33}$ K. Müller-Dethlets, M. Sander, and E. W. Schiag, Z. Naturforsch 39a, 1089 (1984); Chem.

Phys. Lett. 12, 291 (1984); K. Müller-Dethlefs and E. W. Schlag, Ann. Rev. Phys. Chem. 42. 109 (1991).

${ }^{34}$ E. P. Wigner, Phys. Rev. 73, 1003 (1948).

35R. Spohr, P. M. Guyon. W. A. Chupka, and J. Berkowitz, Rev. Sci. Instrum. 42, 1872 (1971).

36T. Baer, W. B. Peatman, and E. W. Schiag, Chem. Phys. Lett. 4, 243 (1969).

37J. D. Watts and R. J. Bartlett, to be published.

${ }^{38} \mathrm{G}$. Herzberg, Electronic Spectra of Polyatomic Molecules, Vol III (D. Van Nostrand Company. Inc., Princeton 1967) p. 176.

${ }^{39}$ K. Raghavachari and J. S. Binkley, J. Chem. Phys. 87, 2191 (1987).

${ }^{40}$ See the $C_{6}$ citing in Ref. (10).

41J. Kurtz and L. Adamowicz, Astrophys. J. 370, 784 (1991).

4zw. C. Lineberger and T. A. Patterson, Chem. Phys. Lett. 13, 40 (1972).

${ }^{43}$ E. A. Brinkman, E. Günther, and J. I. Brauman, J. Chem. Phys. 95.6195 (1991).

44U. Fano, Phys. Rev. 124, 1866 (1961).

${ }^{45}$ R. S. Berry, J. Chem. Phys. 45, 1228 (1966); R. S. Berry and S. E. Nielson, Phys. Rev. 1, 395 (1970).

46J. N. Bardsley, Chem. Phys. Lett. 1, 229 (1967).

"J. Simons, J. Am. Chem. Soc. 103, 3971 (1981). 
46P. K. Acharya, R. A. Kendall, and J. Simons, J. Am. Chem. Soc. 106, 3402 (1984).

${ }^{49}$ R. D. Mead, K. R. Lykke, W. C. Lineberger, J. Marks, and J. I. Brauman, J. Cluem. Phys. 81. 4883 (1984); K. R. Lykke, R. D. Mead, and W. C. Lineberger, Phys. Rev. Lett. 52, 2221 (1984).

50K. R. Lykke, D. M. Neumark, T. Andersen, V. J. Trapa, and W. C. Lineberger, J. Chem. Phys. 87, 6842 (1987).

51K. J. Taylor, C. Jin, J. Conceica, L. Wang, O. Cheshnovsky, B. R. Johnson, P. J. Nordlander, and R. E. Smalley, J. Chem. Phys. 93, 7515 (1990).

52w. R. Garrett, Phys. Rev. A 3, 961 (1971), and reterences therein. 
Table (3.1) Peak positions, relative energies, and assignments for the threshold photodetachment spectrum of $\mathrm{C}_{6}$.

\begin{tabular}{|c|c|c|c|c|}
\hline Peak & $\begin{array}{l}\text { Position } \\
\text { (nm) }\end{array}$ & $\begin{array}{l}\text { Rel. Energy } \\
\qquad\left(\mathrm{cm}^{-1}\right)\end{array}$ & Assignment & $\begin{array}{l}\text { Calculation } \\
\text { (Martin) }^{20}\end{array}$ \\
\hline A & 296.65 & 0 & Origina & - \\
\hline$D$ & 292.41 & $489(10)$ & $3_{0}^{\dagger}$ & 673 \\
\hline $\mathbf{G}$ & 285.45 & $1322(10)$ & $20^{1}$ & 1759 \\
\hline 1 & 279.56 & $2061(10)$ & $1_{0}^{1}$ & 2167 \\
\hline
\end{tabular}

aThis corresponds to an Electron Affinity of $4.180(0.001) \mathrm{eV}$ 
Table (3.2) Peak positions, relative energies, and assignments for the total cross section spectrum of $C_{5}$. The calculated energies are based on an MP2/6-31G" calculation [Ref. (20)] predicting the hamonic frequencies: $v_{1}=2167, v_{2}=1759, v_{3}=673, v_{4}=2009, v_{5}=1244_{1} v_{6}=$ $551, v_{7}=223, v_{8}=432$, and $v_{9}=108$.

\begin{tabular}{|c|c|c|c|c|}
\hline Peak & $\begin{array}{c}\text { Position } \\
\text { (nm) }\end{array}$ & $\begin{array}{l}\text { Rel. Energy } \\
\left(\mathrm{cm}^{-1}\right)\end{array}$ & Assignment & $\begin{array}{c}\text { Calculation } 20 \\
\left(\mathrm{~cm}^{-1}\right)\end{array}$ \\
\hline$a_{n}$ & 297.019 & 0 & Origin & - \\
\hline$b_{n}$ & 295.386 & $186(2)$ & $9{ }_{0}^{2}$ & 216 \\
\hline$c_{n}$ & 293.622 & $390(2)$ & $70^{2}$ & 446 \\
\hline$d_{n}$ & 292.84 & $480(2)$ & $30^{1}$ & 673 \\
\hline$e_{n}$ & 291.60 & $626(2)$ & $B_{0}^{2}$ & 862 \\
\hline$t_{n}$ & 288.77 & $962(3)$ & $3_{0}^{2}$ & 1346 \\
\hline$g_{n}$ & 285.92 & $1307(5)$ & $20^{3}$ & 1759 \\
\hline$h_{n}$ & 282.95 & $1674(5)$ & $5_{0}^{2}$ & 2488 \\
\hline$i_{n}$ & 279.91 & $2058(5)$ & $10^{1}$ & 2167 \\
\hline in & 279.87 & $2063(5)$ & $5_{0}^{2} 7_{0}^{2}$ & 2934 \\
\hline$k_{n}$ & 279.17 & $2153(5)$ & $5_{0}^{2} 3_{0}^{1}$ & 3161 \\
\hline$I_{n}$ & 276.28 & $2527(5)$ & $1_{0}^{1} 3_{0}^{1}$ & 2840 \\
\hline$m_{n}$ & 270.31 & $3327(5)$ & $10{ }^{\prime} 20^{\prime}$ & 3926 \\
\hline
\end{tabular}


Table (3.3) Positions, relative energies and proposed assignments for the peaks found within the SA manitold.

\begin{tabular}{|c|c|c|c|}
\hline Peak & $\begin{array}{c}\text { Position } \\
\text { (nm) }\end{array}$ & $\begin{array}{l}\text { Rel. Energy } \\
\qquad\left(\mathrm{cm}^{-1}\right)\end{array}$ & Assignment \\
\hline$a_{n}$ & 297.019 & 0 & Origin \\
\hline$a_{1}$ & 297.179 & $-18.1(1)$ & $9_{1}^{1}$ \\
\hline as & 297.241 & $-25.1(2)$ & $7 !$ \\
\hline$a_{3}$ & 297.277 & $-29.2(2)$ & S-O \\
\hline$e_{\Delta}$ & 297.317 & $-33.7(3)$ & $g_{2}^{2}$ \\
\hline$a_{5}$ & 297.380 & $-40.9(3)$ & $7_{1}^{1} 9_{1}^{1}$ \\
\hline$a_{f}$ & 297.439 & $-47.5(3)$ & $g_{1}^{\top}$ plus $\mathrm{S}-\mathrm{O}$ \\
\hline$a_{7}$ & 297.509 & $-55.5(3)$ & $7_{1}^{1} 9_{2}^{2}$ \\
\hline$a_{A}$ & 297.582 & $-63.7(3)$ & $9_{2}^{2}$ plus S-O \\
\hline
\end{tabular}


Table (3.4) Peak intensity changes between the one-photon and two-color, two-photon scans in the SA and SD manifolds.

$\begin{array}{ccc}\text { Peak } & \begin{array}{c}\text { Wavelength } \\ (\mathrm{nm})\end{array} & \begin{array}{c}\text { Intensity_blue+green) } \\ \text { Intensity (blue only) }\end{array} \\ a_{n} & 297.019 & 3.0 \\ a_{1} & 297.179 & 1.1 \\ a_{7} & 297.254 & 1.5 \\ a_{3} & 297.277 & 1.8 \\ a_{6} & 297.439 & 1 \\ a_{7} & 297.509 & 1.9 \\ d_{0} & 292.84 & 1.6 \\ d_{1} & 292.99 & 1 \\ d_{2} & 293.09 & 1.9\end{array}$


Figure (3.1) Enargy level diagram illustrating autodetachment and threshold photodetachment transitions. The transitions to the neutral levels yield the zero-kinetic-energy electrons [Eqn. (3.1)] and the transitions to the excited anion levels are followed by autodetachment [Eqn. (3.2)].

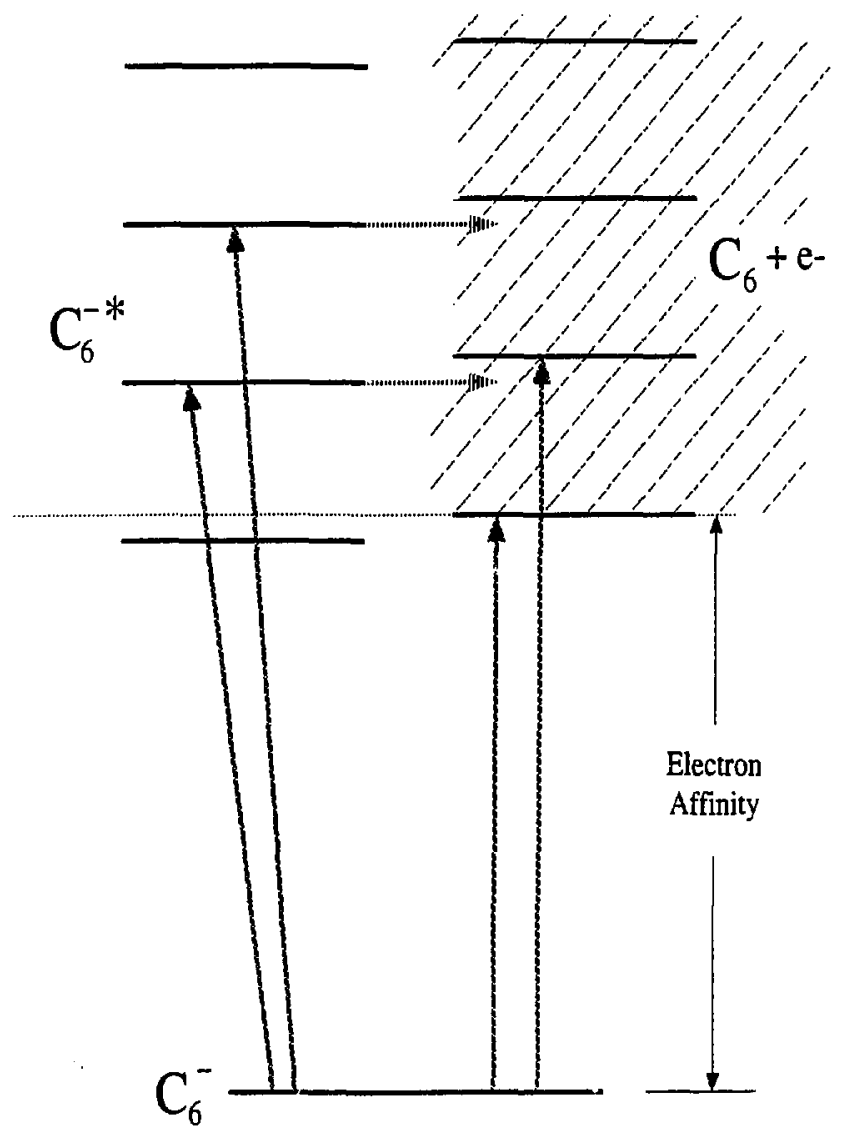


Figure (3.2) Diagram of the tunable photodetachment instrument: (A) interaction region for threshold photodetachment; $(B)$ interaction region for the total cross section experiment; (1) detachment wondows; (2) electron extraction plates; (3) magnetic shielding; (4) electron Einzel lens; (5) ion detector. Electron detector is located directly above the $\otimes$.

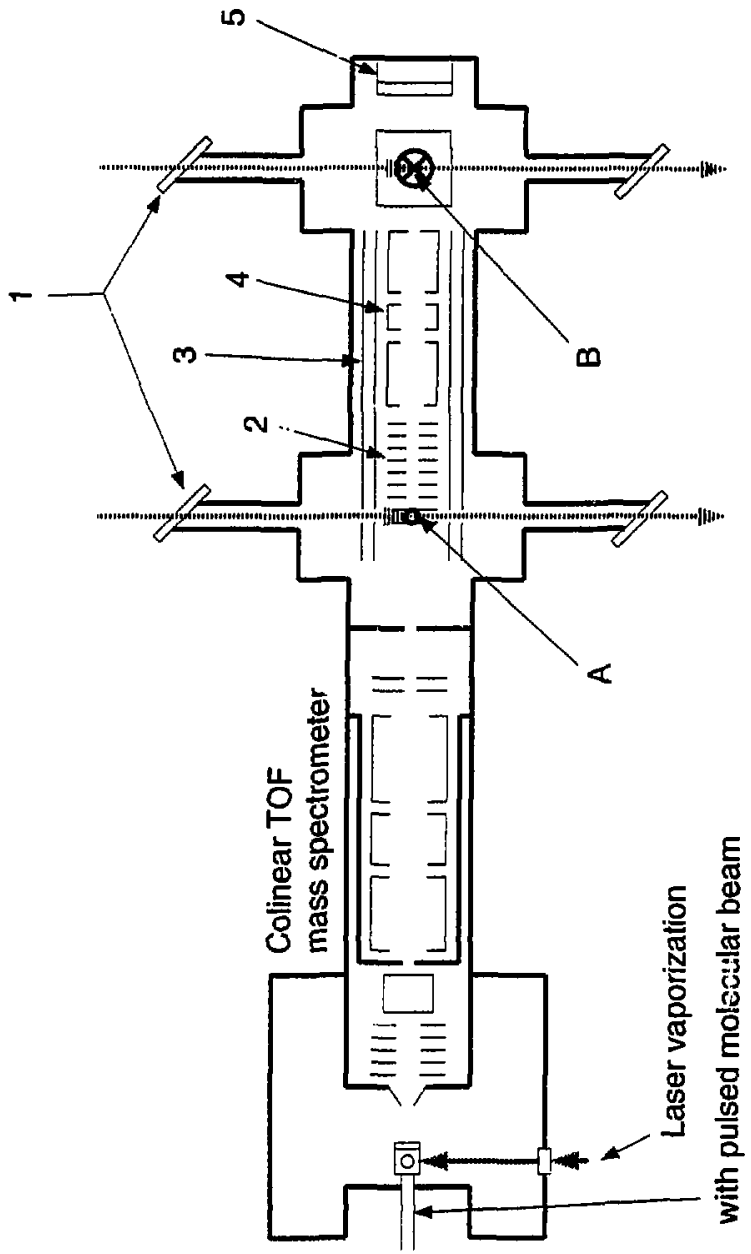


Figure (3.3) Idealized appearance of photodetachment signal in the absence of autodetachment for the three modes of operation of the instrument: (a) threshold photodetachment spoctrum, (b) "partially discriminated" spectrum, and (G) total photodetachment cross section.

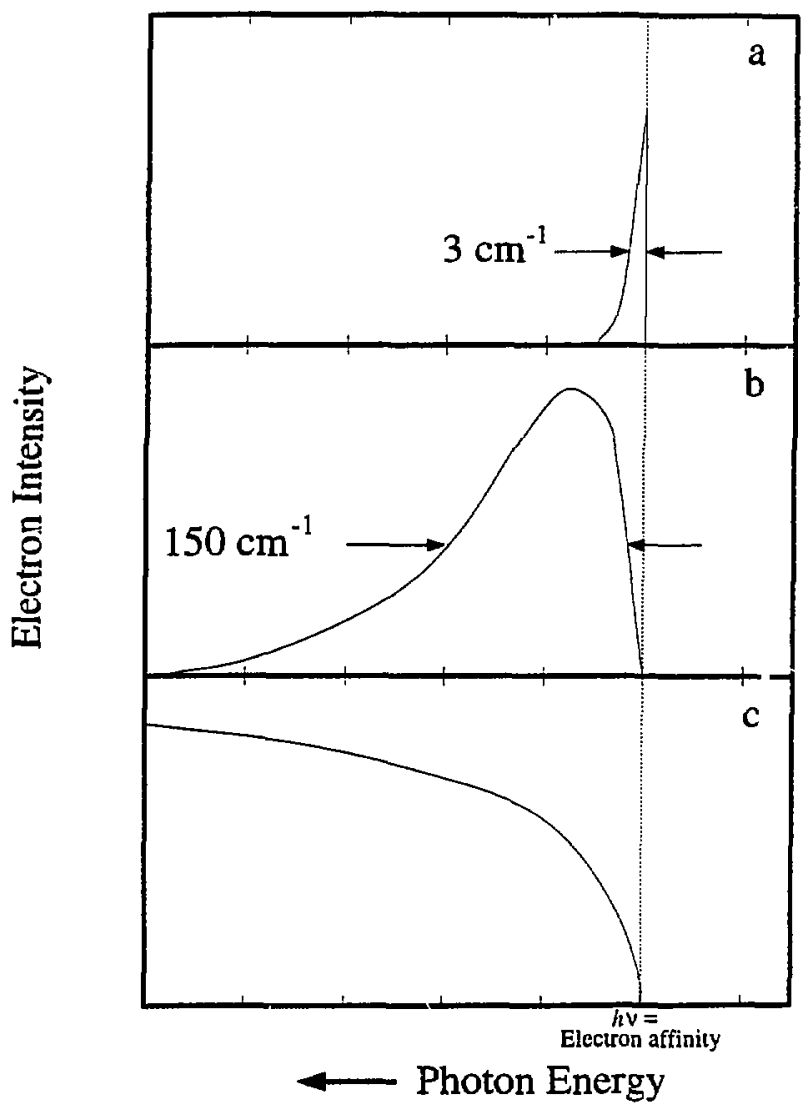


Figure (3.4) (a) Threshold photodetachment spectrumi of $\mathrm{C}_{6}^{-}$. (b) Partially discriminated photodetachment cross section spectrum of $\mathrm{C}_{6}{ }^{-}$. (c) Total photodetachment cross section of $\mathrm{C}_{6}{ }^{-}$

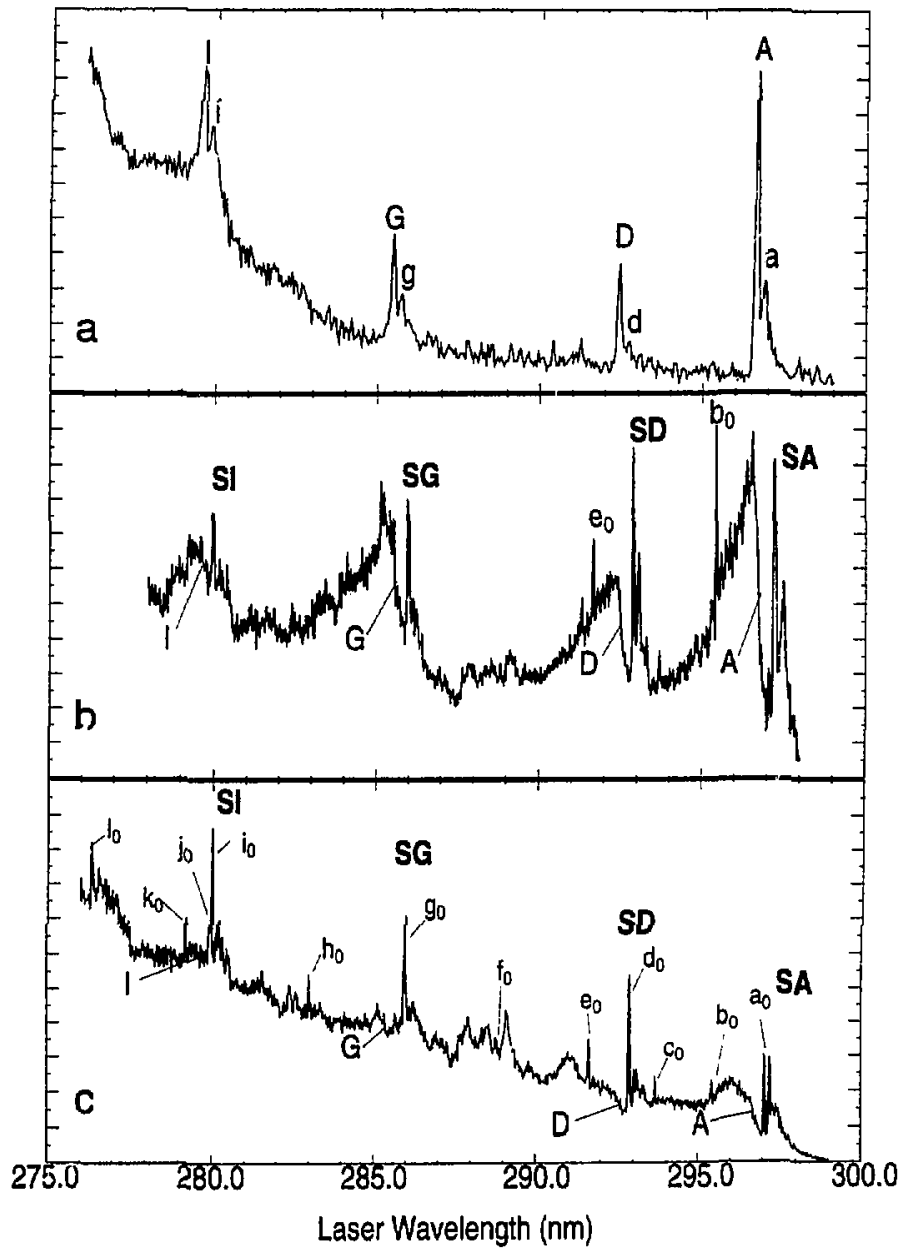


Figure (3.5) (a) Expanded-scale, finer-step scan of the SA manifold obtained in partial discrimination mode. (b) Expanded-scale, finer-step scan of the SA manifold obtained in total cross section mode.

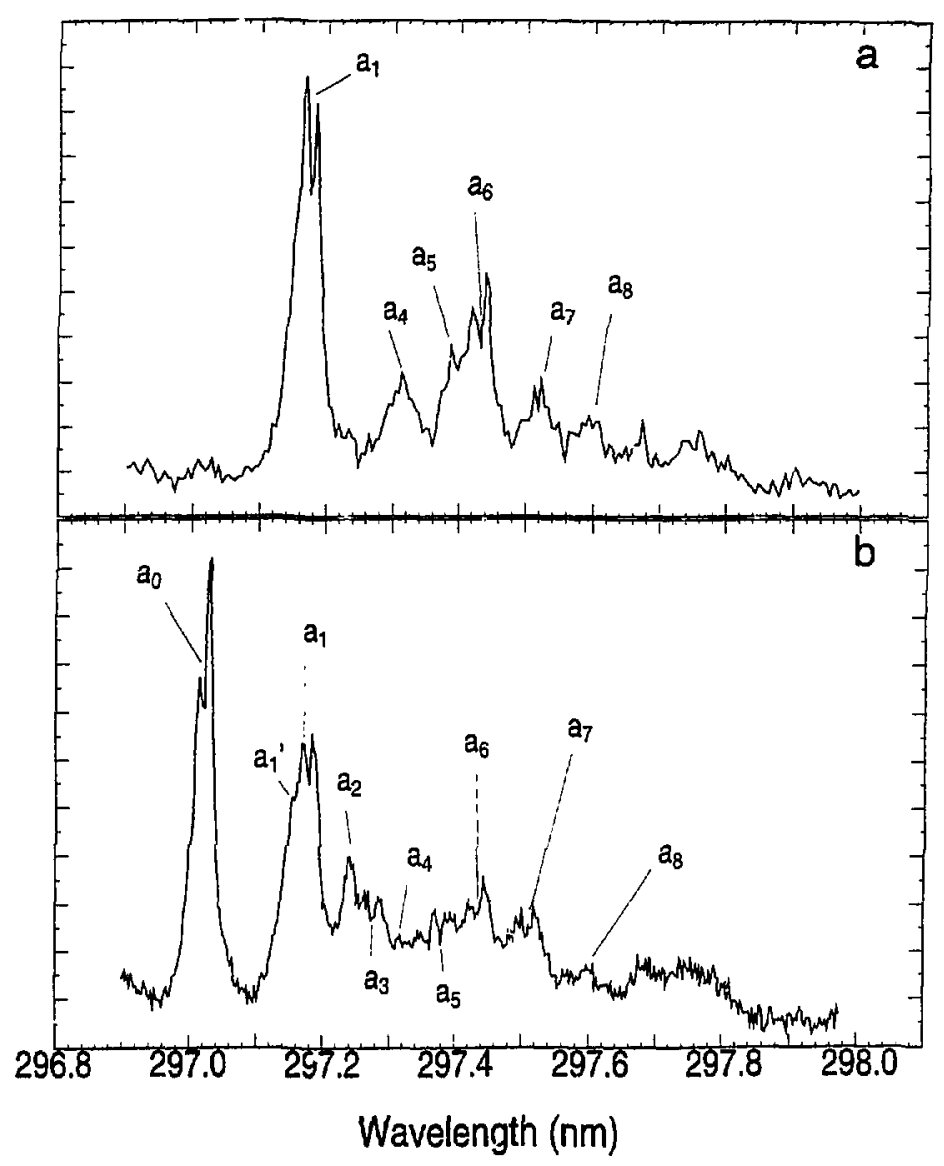


Figure (3.6) (a) Expanded-scale, finer-step scan of the SD manifold obtained in partial discrimination mode. (b) Expanded-scale, finer-step scan of the SD manifold obtained in total cross section mode.

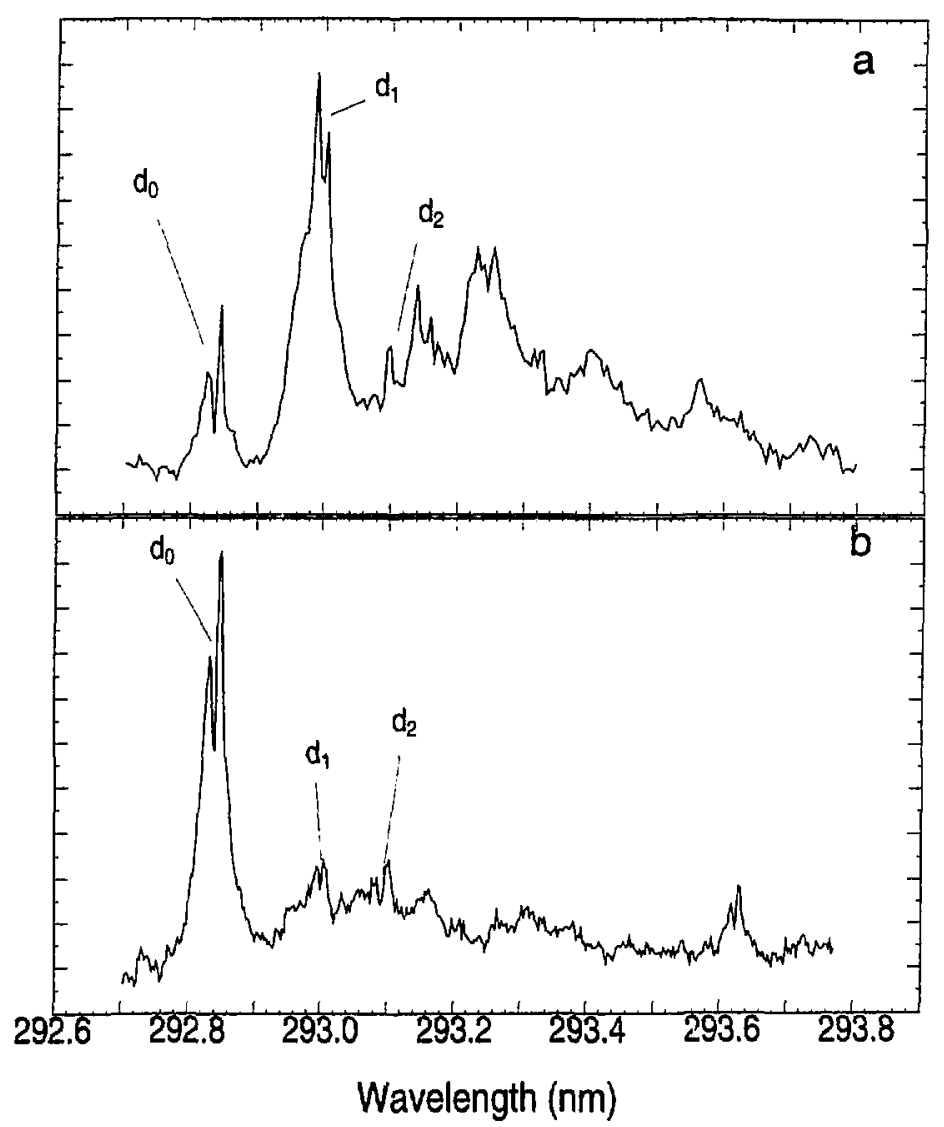


Figure (3.7) (a) Total photodetachment cross section spectrum of $\mathrm{C}_{6}{ }^{-}$obtained under nomal ion source conditions. (b) Total photodetachment cross section spectrum of $\mathrm{C}_{6}{ }^{-}$obtained from ions generated with low carrier gas pressure.

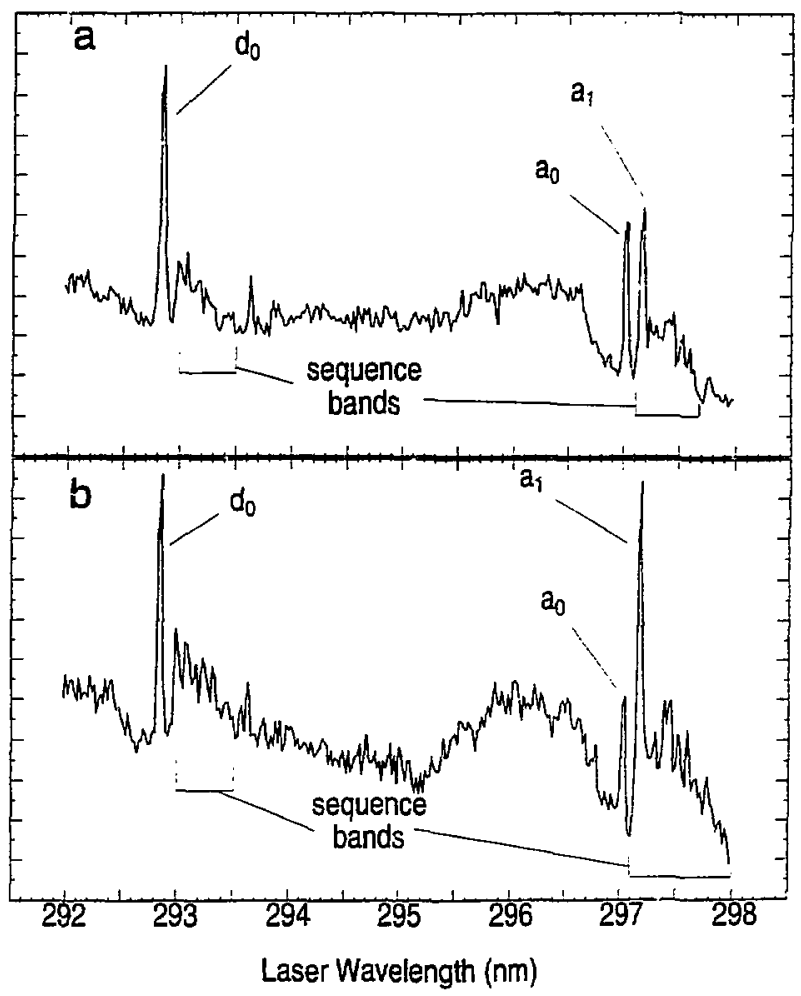


Figure (3.8) Anion photodetachment as a function of detachment pulse energy for peaks $a_{0}$ and $a_{2}$ in the SA manifold.

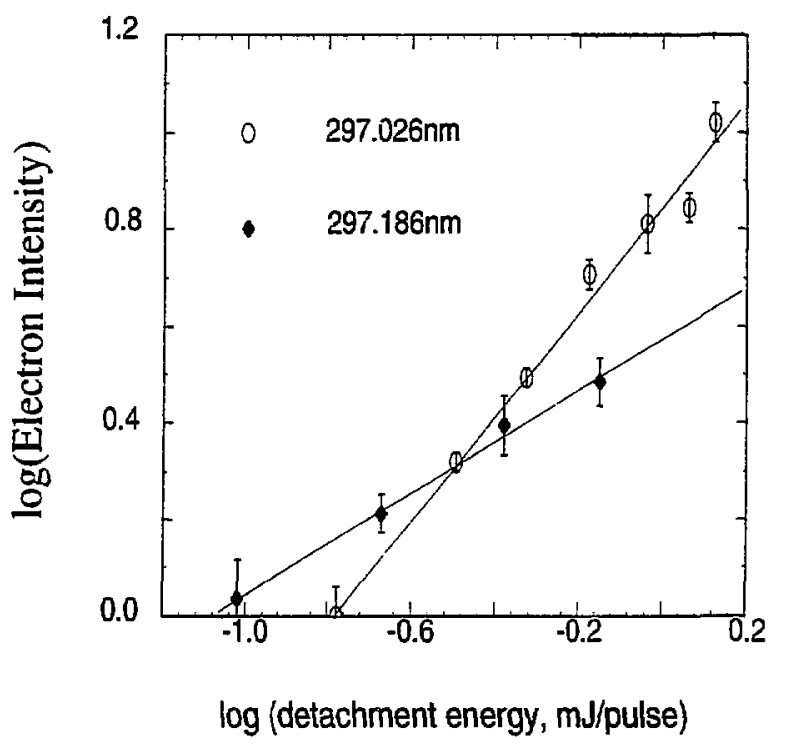


Figure (3.9) (a) Two-photon, two-color scan (solid line) of SA manitold using $0.5 \mathrm{~mJ} / \mathrm{pulse}$ of tunable UV light and $2 \mathrm{~mJ} /$ pulse of $532 \mathrm{~nm}$ light. The superimposed spectrum (circles) show same spectrum in the absence of the $532 \mathrm{~nm}$ light. (b) Sarne for SD manifold.
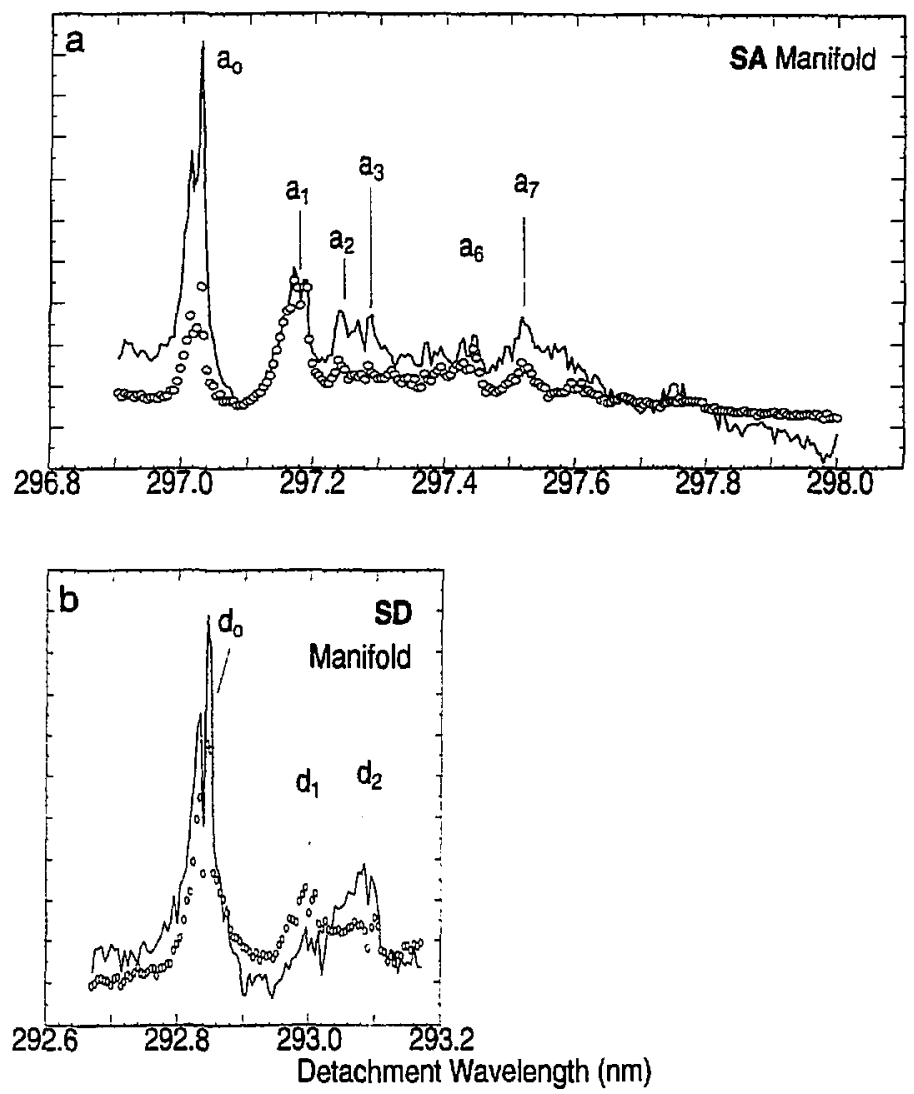
Figure (3.10) Parameters for the one-dimensional vibrational calculation for $\mathrm{C}_{6}$ with the three symmetric and two antisymr it ic stretch normal coordinates.

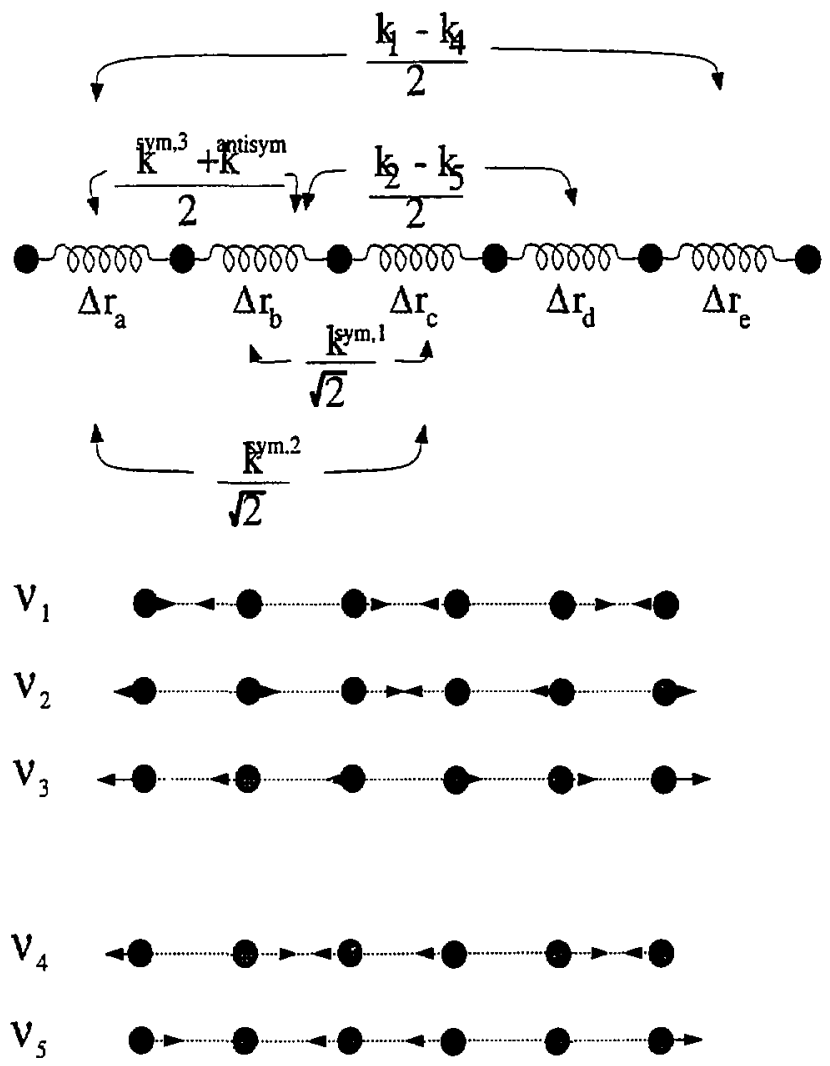


Figure (3.11) Franck-Condon simulation of the threshold photodetachment spectrum of $\mathrm{C}_{6}$ using symmetric stretch normal coordinate displacements described in text.

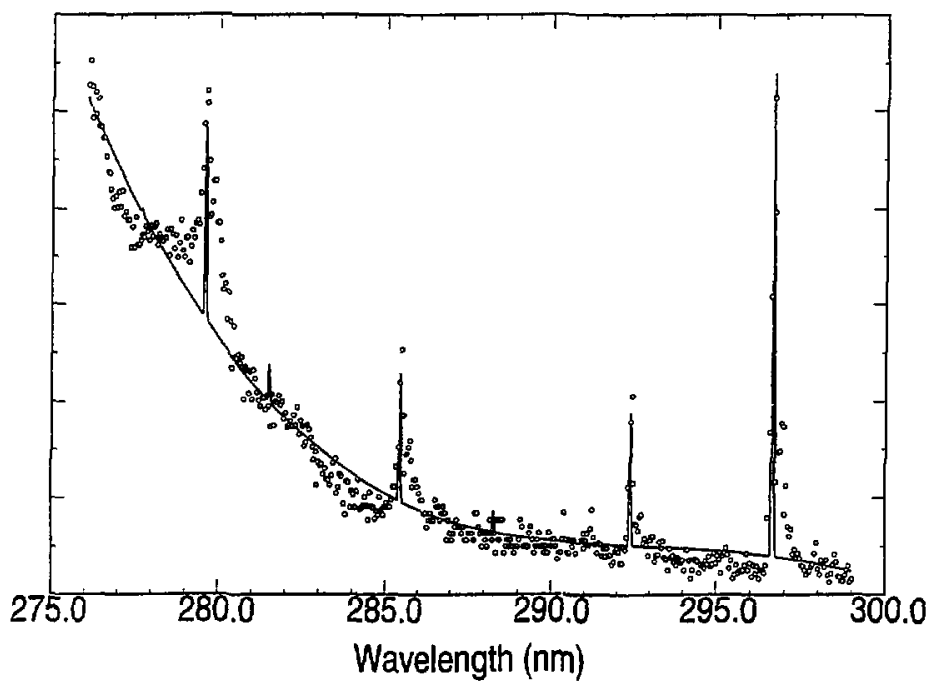


Figure (3.12) Possible explanations for peaks in the $C_{6}{ }^{-}$photodetachment cross section at photon energies below the detachment threshold [SA menifold, Figure (3.4c)]. (a) Peaks are hot bands from vibrationally excited $\mathrm{C}_{6}{ }^{-}$. (b) Peaks are from transitions to bound states which are subsequently photodetached by absorption of a second photon.
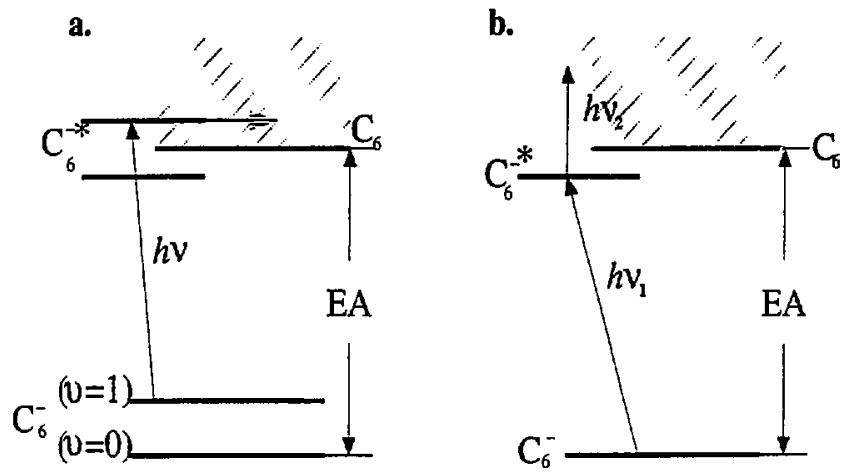


\section{Chapter 4. Structure in the total photodetachment cross sections of $C_{n}-(n=4,6-9)$}

\section{Introduction}

In the previous chapter, sharp structure in the total photodetachment cross section of $\mathrm{C}_{6}{ }^{-}$due to autodetachment or two-photon detachment of an excited $\mathrm{C}_{6}{ }^{-}$state was presented. ${ }^{t}$ Similar sham structure in the cross section of $\mathrm{C}_{2}{ }^{-}$has been previousiy observed by Lineberger. ${ }^{2}$ In the case of $\mathrm{C}_{2}{ }^{-}$, an excited valence state of the anion is bound with respect to the detachment continuum, but autodetachment becomes energetically possible in the vibrationally excited levels of the excited anion state. This is the same scenario that has been proposed for $\mathrm{C}_{6}{ }^{-}$, but the excited anion state is much closer to the detachment cortinuum in $\mathrm{C}_{6}{ }^{-}$than in $\mathrm{C}_{2}{ }^{-}$. While no sharp structure has been observed in the total photodetachment cross sections for any small carbon cluster anions other than $C_{2}^{-}$and $C_{6}{ }^{-}$, broad features observed in the $C_{4}^{-}, C_{6}{ }^{-}, C_{7}, C_{9}{ }^{-}$ and $\mathrm{C}_{9}{ }^{-}$cross section spectra will be the focus of this chapter.

Bound or quasibound anion states have been observed for a number of molecules, and the nature of these excited anion states can take one of several different forms. They can be valence-bound excited states, such as with $\mathrm{C}_{2}^{-}$. Large organic molecules such as $\mathrm{TCNQ}^{3}$ can also have bound excited anion states because of large unfilled orbitals. The general criteria for 
bound valence states are that the anion is open-shell, which allows for close-lying electronic states, and higher electron affinities, which allows low-lying states to be bound with respect to the detachment continuum. There are also electrostatically bound states (EBS). For instance, excited but bound anion states can exist in situations where the corresponding neutral has a large enough dipole to weakly bind an electron in a diffuse dipolar ortital. These dipole bound anion states have been observed in species such as $\mathrm{NH}^{-},{ }^{4} \mathrm{C}_{2} \mathrm{H}_{3} \mathrm{O}^{-}, 5 \mathrm{FeO}^{-6} \mathrm{CH}_{2} \mathrm{CN}^{-}$and $\mathrm{PtN}^{-7}$ If the neutral core lacks a dipole but is especially polarizable, an image charge bound (ICB) anion state can exist, as is the case with the $\mathrm{Au}_{6}{ }^{-}$ing. ${ }^{8}$ These electrostatically bound states are usually bound by around $100 \mathrm{~cm}^{-1}$ with respect to the detachment continuum, and have vibrational frequencies very similar to the neutral core.

Bound excited valence states of anions in general are not common. They are, however, feasible in these linear carbon clusters, particularly the even-numbered species, because of their high electron affinity and unfilled $\pi$ orbitals. It may therefore seem surprising that no sharp autodetachment features have been observed for $\mathrm{C}_{4}{ }^{*}$ and $\mathrm{C}_{8}{ }^{-}$, which are also open-shelled and have high electron affinities. However, the extra electron in the ground electronic states of $\mathrm{C}_{4}^{-}$ and $\mathrm{C}_{8}{ }^{-}$is in an antibonding $\pi_{9}$ orbital, while for $\mathrm{C}_{2}^{-}$and $\mathrm{C}_{6}{ }^{-}$, the electron is in bonding orbitals ( $\sigma_{g}$ for $\mathrm{C}_{2}{ }^{*}$ and $\pi_{4}$ for $\mathrm{C}_{6}{ }^{-}$), making the excitation energetics different for these various species. For comparison, Table (4.1) summarizes the neutral and anion electronic orbital occupancies for the small carbon cluster chains ${ }^{9}$ along with the neutral electron affinities. The electron affinities for $C_{5}$ and $C_{6}$ are from the previously obtained ZEKE spectra of $C_{5}{ }^{-}$and $C_{6},{ }^{-1,10}$ the remaining electron affirities are from the fixed-frequency photoelectron spectra of the carbon cluster anions. ${ }^{11}$

The principle purpose of this chapter is to present the structure observed in the total photodetachment cross sections of $C_{n}^{-}(n=4,6-9)$. The spectra themselves and the qualitative discussions on the spectral structure that follow can serve as a starting point for future studies on the electronic properties of small carbon cluster anions. Most of the structure 
presented below is very broad, and seems to be more indicative of anion states lying above the detachment continuum. However, for $\mathrm{C}_{6}, \mathrm{C}_{7}^{-}$and $\mathrm{C}_{9}{ }^{-}$, spectral features coincide with detachment thresholds, which suggests some interesting interaction between the electron and the neutral core near the continuum.

\section{Experimental}

The apparatus used to obtain the total photodetachment cross section scans has been described in detail previously (Chapter 3 , Sec. II), but the basic operation is as follows. The carbon cluster anions, neutrals, and cations are generated using a laser-vaporization/pulsed molecular beam source based on a design developed by Smalley. ${ }^{12}$ The second harmonic output of a Nd:YAG laser (2 to $6 \mathrm{~mJ} /$ pulse) operated at a $20 \mathrm{~Hz}$ repetition rate is focused onto the surface a rotating, translating carbon rod. The resulting plasma is entrained in a pulse of helium camier gas from a General Valve solenoid-type molecular beam valve, and is expanded through a $0.25^{n}$ long channel where clustering and cooling occurs. Upon expanding from the channel, the negative clusters that pass through a $2 \mathrm{mrn}$ skimmer are colinearly accelerated to 1 $\mathrm{keV}$, and separated out by mass in a $1 \mathrm{~m}$ beam-modulated TOF mass spectrometer. The massseparated clusters then pass through a $5 \mathrm{~mm}$ defining slit into a detector region, where the negative ion of interest can be selectively photodetached using a $\mathrm{XeCl}$ excimer-pumped tunable dye laser. The ions are detached in a constant field of approximately $2 \mathrm{~V} / \mathrm{cm}$, which immediately extracts the detached electrons to a dual microchannel plate detector. The electron signal is collected as a function of laser wavelength, and is normalized to the laser power and ion signal.

For detachment of $\mathrm{C}_{4}^{-}$, Rhodamine 610, Rhodamine 590, and Coumarin 540 laser dyes

were used. For $\mathrm{C}_{6}{ }^{-}$, Rhodamine 590 and Coumarin 540 laser dyes were used for the abovethreshold spectra, and QUI was used for the below-threshold spectra. QUI was also used in 
obtaining the $\mathrm{C}_{7}^{-}$spectrum, and QU! and DMQ were used to obtain the $\mathrm{C}_{8}{ }^{-}$spectrum. The QUI and pTP dyes were used for the $\mathrm{C}_{9}{ }^{-}$spectrum.

\section{Results}

The total photodetachment cross section spectra of $\mathrm{C}_{4}^{-}$and $\mathrm{C}_{6}{ }^{-} \cdot \mathrm{C}_{9}{ }^{-}$?re presented as the solid lines in Figures (4.1) - (4.3). The spectra are grouped according to their common features. In the $\mathrm{C}_{7}{ }^{-}$and $\mathrm{C}_{9}{ }^{-}$spectra, shown in Figure (4.1), the structure appears very near the detachment continuum. There is also structure found $2700 \mathrm{~cm}^{-1}$ to lower photon energy in the $\mathrm{C}_{9}{ }^{-}$spectrum. In the $\mathrm{C}_{4}^{-}$and $\mathrm{C}_{6}^{-}$spectra, shown in Figure (4.2), there is bioad structure above the detachment continuum (along with the shap strurture in the case of $\mathrm{C}_{6}{ }^{-}$, as was discussed in Chapter 3). The $\mathrm{C}_{8}{ }^{-}$and also $\mathrm{C}_{6}{ }^{-}$spectra in Figure (4.3) show broad structure around $1 \mathrm{eV}$ belnw the detachment continuum. On all of the spectru, the $4.65 \mathrm{eV}$ PES have been superimposed as a dashed line onto the detachment wavelength (nm) scale of the total photodetachment cross section spectra (solid lines). ${ }^{11}$ The most intense peak in the PES is the origin transition, and thus serves as a guide to where the detachment continuum is located.

Some of the structure in the cross section spectrum of $\mathrm{C}_{7}{ }^{-}$[Figure (4.1), upper panel] is fairly sharp, and peak $A_{1}$ appears to be right at the detachment continuum. Peaks $A_{1}$ and $A_{2}$, spaced by $244 \mathrm{~cm}^{-1}$, are actually partially resolved doublets with a characteristic spacing of approximately $25 \mathrm{~cm}^{-1}$. The $A_{1}$ doublet is $30 \mathrm{~cm}^{-1}$ wide full width at half maximum (FWHM), and $A_{2}$ is $40 \mathrm{~cm}^{-1}$ wide FWHM. Peak $B$ is approximately $150 \mathrm{~cm}^{-1}$ wide and is approximately 200 $\mathrm{cm}^{-1}$ to the red of peak $A_{1}$. The peak positions and relative energies are listed in Table (4.2).

The total cross section of $\mathrm{C}_{9}^{-}$[Figure (4.1), bottom panel] in the region of the detachment continuum is overlaps with, and is startlingly similar to the PES. Peaks $A_{1}$ and $A_{3}$ exhibit the $496 \mathrm{~cm}^{-1}$ vitrational spacing observed in the PES. There is also a shoulder, $A_{2}$. found $200 \mathrm{~cm}^{-1}$ to the blue of $A_{1}$. The $350 \mathrm{~cm}^{-1}$ wide (FWHM) peaks in the total cross section 
are slightly broader than those in the PES. Additional strucfure is found below the detachment continuum; peaks $B_{1}, B_{2}$ and $B_{3}$ form a $420 \mathrm{~cm}^{-1}$ progression, and the energy spacing between $B_{1}$ and $A_{1}$ is $0.34 \mathrm{eV}\left(2720 \mathrm{~cm}^{-1}\right)$. Peaks $B_{n}$ are $250 \mathrm{~cm}^{-1}$ wide and appear to have some additional, only partially resolved fine structure spaced by $90 \mathrm{~cm}^{-7}$. Although the peaks are very broad, it does appear that the $420 \mathrm{~cm}^{-1} \mathrm{~B}_{\mathrm{n}}$ spacing corresponds to a different (anion) state than does the $496 \mathrm{~cm}^{-1}$ piogression. Peak positions and relative energies for the tolal cross section of $\mathrm{C}_{9}{ }^{-}$are listed in Table (4.3).

The structure observed in the total cross section of $\mathrm{C}_{4}^{-}$[Figure (4.2), top panel] starts approximately $1700 \mathrm{~cm}^{-1}(0.21 \mathrm{eV})$ abcve the detachment continuum. The structure is broad and irregular, and is on the rising slope of the background signal of the apparatus. The positions and relative energies of the structure are listed in Table (4.A). While there is no obvious pattem to the structure, peaks $A_{2}, A_{3}$ and $A_{4}$ may form a pregression with a spacing on the order of 700 $10800 \mathrm{~cm}^{-1}$. Peak $A_{3}$ is $90 \mathrm{~cm}^{-1}$ wide, FWHM.

The shap structure in the $\mathrm{C}_{6}{ }^{-}$total photodetachment cross section [shown in the lower panel of Fig'ure (4.2)], as mentioned before, has been elaborated upon in Chapter 3. However, there are several broad features that were not discussed. For instance, just above the origin threshold and the threshold for a vibrational level are approximately $200 \mathrm{~cm}^{-1}$ wide bumps. labeled $A_{1}$ and $A_{2}$. These are obviously correlated with the detachment thresholds. There is also a distinct triplet, labeled $T$, found approximately $1800 \mathrm{~cm}^{-1}$ above the detachment continuum. The spacing between the peaks in the triplet $T$ is approximately $70 \mathrm{~cm}^{-4}$. The positions and relative energies of this structure are listed in Table (4.5).

Table (4.5) aiso lists the broad structure observed in the total cross section of $\mathrm{C}_{6}{ }^{-}$found almost $1 \mathrm{eV}$ below the detachment continuum, which is showr, in the top panel of Figure (4.3). Peak $A_{1}$ is at a $3.22 \mathrm{eV}$ detachment energy and the electron affinity of $C_{6}$ is $4.180 \mathrm{eV}$. The structure is roughly in three clumps spaced by approximately $1000 \mathrm{~cm}^{-1}$. 
Similar structure was observed $1 \mathrm{eV}$ below the delachment continuum for $\mathrm{C}_{3}$, as is shown in the lower panel of Figure (4.3). Peak $A_{1}$ is at $3.33 \mathrm{eV}$ detachment energy. and the electron affinity of $C_{8}$ is $4.39 \mathrm{eV}$. As with several other clusters, peak $A_{1}$ is a partialiy resolved doublet with a splitting of $44 \mathrm{c}: n^{-1}$. Peak $A_{2}$ has partially resolved structure as well, and $A_{1}, A_{2}$ and $A_{3}$ appear to form an approximately $590 \mathrm{~cm}^{-1}$ progression. The remaining broad structure is listed in Table (4.6). It is worth noting that the lower-intensity $A_{7}$ feature is an entirely reproducible zroup of $18 \mathrm{sm} \mathbf{r}^{-1}$-wide peaks in a configuration reminiscent of the trip!et $(T)$ found in the total cross section spectium of $C_{6}$ [Figure (4.2), bottom panel] except that the energy spacing of the $\mathrm{C}_{8}{ }^{-}$peaks are approximateiy half that of the $\mathrm{C}_{6}{ }^{-}$triplet.

\section{Analysis}

Schenatics oi the several processes that could result in broad structure in the total photodetachment cross section scans are shown in Figure (4.4). Cases (a) and (b) show onephoton transitions that could result in sroad structure above and below the detachment continuum, respectively. The first (a) shows transitions from the ground anion state to a quasi. hound excited anion state that lies above the detachment continuum, and autodetaches quickly, which would result in hroad (depending on the lifetime of the autodetaching state) structure that

lies just above the dztachment continuum. The second scenario (b) shows transitions from a low-lying excited anion state to a quasi-bound anion state which quickly autodetaches. This model could explain broad structure slightly below the detachmo:nt continuum and possibly above the threshold as wall.

Cases (c) and (d) in Figure (4.4) are two-photori processes, which are possible given adequate laser power. Case (c) is a scenario in whicn the ground anion state absorbs one photon in a transition to a bound excited anion state, which is followed by Jetachment by a second photon tc the detachment continuum. This is the type of process respcisible for sorrie 
of the sharp structure observed below the detachment continuum in the $\mathrm{C}_{6}^{-}$and $\mathrm{C}_{2}^{-}$total photodetachment cross sections, but also for the broader structure below threshoid for larger organic molecules. 3 However, given the simplicity and high symmetry in the small carbon clusters, it doesn't seem likely that this process could generate broad structure well below detachment threshold. In case (d), the anion absorbs two photons. the sum of the two photons being resonant with a very high-lying quasi-bound anion state which very quickly autodetaches. This process, though it should be much weaker than the other three described above, would definitely yield broad structure found well below the detachment continuum.

There may be other, more complicated processes occurring as well, such as photofragmentation followed by detachment. These possibilities will not be addressed in this chapter. Again, the total cross section spectra presented here are to be taken as starting points for future work, but several observations regarding each spectrum will be made here.

$c_{7}^{-}$cross section: The relatively sharp structure observed in this spectrum is reminiscent of the sharp two-photon and autodetachment features observed in the $\mathrm{C}_{6}^{*}$ total cross section spectrum [lower panel of Figure (4.2) and Chapter 3], although it is a factor of four broader. It is difficult to deternine from the superimposed PES whether the anion state lies above or below the detachment continuum, but the observation that peak $A_{1}$ is less intense than $A_{2}$ suggests that $A_{1}$ may be from a two-photon detachment process [case (c) in Figure (4.4)] whereas peak $A_{2}$ is from a one-photon process [case (a) in Figure (4.4)]. The doublet profile of peak $A_{1}$ likely reflects the spin-orbit splitting in the anion; it is similar to the spin-orbit splitting in $C_{5}^{-}$and $C_{6}^{-}$. The spacing between peaks $A_{1}$ and $A_{2}, 218 \mathrm{~cm}^{-1}$, could be two quanta in a lowfrequency bend mode. The broad feature labeled $\mathrm{B}_{1}$ does not appear to have any connection to $A_{1}$ and $A_{2}$, and it may possibly be an electronic hot hand as depicted in case (b), a two-photon process depicted in case (d) of Figure (4.4), or another more complicated process. 
$\mathrm{C}_{\mathrm{g}^{-}}$crose section: The peaks near the detachment continuum in the $\mathrm{C}_{9^{-}}$total cross section scan are much more broad than those in the $C_{7}$ - spectrum, and therefore most likely to be transitions of type (a) in Figure (4.4). The lifetimes of the excited anion states are obviously short. While peaks $A_{1}, A_{2}$, and $A_{3}$ are nearly equally spaced, the profile of these three peaks does not suggest a vibrational progression. Rather, $A_{3}$ appears to correlate with the lowest frequency symmetric stretch of the neutral, and $A_{2}$ is likely a transition to two quanta in a lowfrequency bend mode. It is remarkable that the cross sections of both $\mathrm{C}_{9}{ }^{-}$and $\mathrm{C}_{7}{ }^{-}$have features that line up so closely with the neutral $\leftarrow$ anion origin in the PES. The width of the features in the cross section argue against an electrostatically bound state, which is necessarily bound with respect to the detachment continuum and would yield $5-6 \mathrm{~cm}^{-1}$ wide structure (convoluting the resolution of the laser with the expected rotational manifold). However, for two (three, including $\bar{\Sigma}_{6} ;$, see below) carbon cluster anions to have quasibound excited states nearly coinciding with the respective detachment continua must be more than a coincidence.

One possibility stems from the effective potential between the electron with the neutral,

$$
V_{\text {eft }}=\frac{-\alpha \alpha}{r^{4}}+\frac{\ell(\ell+1)}{r^{2}}
$$

where the first term is the electrostatic attraction between the electron and the carbon cluster having polarizability $\alpha$, and the second term is the centrifugal contribution to the effective potential from the angular momentum of the photoelectron, $\ell$. For $\ell>0$ (p, d, ...-wave elect:ons), there is some barrier in the potential, which, depending on $\alpha$ and $\ell$ (for larger $\alpha$, the barrier is lower and at greater $r$, and for smaller $\alpha$, the barrier can be higher and closer to the neutral core), might support a quasibound resonance. Such resonances have been observed in atomic ionization cross sections, and while the atomic quasibound neutral resonances are on the order of $\mathrm{eV}$ wide (versus the $0.01 \mathrm{eV}$ wide features in the $\mathrm{C}_{9}$ cross section), in the situation described here, the quasibound level would be very near threshold, so the electron wouldn't be 
extraordinarily energetic, and the barrier might be broad. This explanation is wildly speculative, and should be treated with appropriate caution.

The band at lower detachment energy may be an electronic hot band, with transitions of type (b) in Figure (4.4), or a two-photon process such as case (d). If case (b), the $420 \mathrm{~cm}^{-1}$ spacing between $B_{1}, B_{2}$ and $B_{3}$ may again be activation in the lowest-frequency symmetric stretch of the upper state, or, if either the lower or upper excited anion states are Renner-Teller distorted, it could be a relatively extended progression in a b:snd mode.

$\mathrm{C}_{4}$ - cross section: Not much can be said regarding the broad and irregular structure above the detachment continuum observed in the $\mathrm{C}_{4}^{-}$cross section spectrum. This structure must be due to transitions of type (a) depicted in Figure (4.4). Peaks $A_{2}, A_{3}$ and $A_{4}$ appear to have some similar combination structure tailing to higher detachment energy. Otherwise, no other pattems are obvious.

$\mathrm{C}_{6^{-}}$cross section: The broad bumps found above the detachment continuum of $\mathrm{C}_{6}{ }^{-}$ labeled $A_{1}$ and $A_{2}$ in the $C_{6}-$ total cross section spectrum [bottom panel, Figure (4.2)]. Their close correlation with detachment thresholds is similar in nature to the structure in the $\mathrm{C}_{9}$ spectrum. The total cross section of $\mathrm{C}_{5}^{-}$(not shown), while much simpler than the cross sections of $\mathrm{C}_{4}{ }^{-}$and $\mathrm{C}_{6}$ through $9^{-}$, also exhibited a broad but distinct bump just to the blue of the sharp detachment threshold. Clearly, some electrostatic effects are affecting the cross section.

The structure observed well below the detachment continuum of $\mathrm{C}_{6} \cdot$ [upper panel, Figure (4.3)] is particularly broad and noisy, and are most likely due to transitions of type (d) in Figure (4.4). Energetically, it is possible that a resonant process [case (b)] is occurring, but the broadness of the features argue against this. Moreover, two-color scans in which the second photon (532 nm, 2nd harmonic output of a YAG) plus the blue photon was sufficient to detach $\mathrm{C}_{6}-$ were performed, and no enhancement of the structure was observed. If the process were of type (c), the color of the second photon would be irrelevant, so long as it was cidequate for 
detachment, in which case the addition of the second photon should result in signal enhancemcit (see Chapter 3, Sec. III and Sec. IV.B.2).

Based on this, the upper state must lie $2.28 \mathrm{eV}$ above the detachment continuum. Several additional points are worth noting. Peaks $A_{1}, A_{3} / A_{4}$, and $A_{6} / A_{7}$ form a $900 \mathrm{~cm}^{-1}$ progression, but because of the nature of the transition, it reflects an $1800 \mathrm{~cm}^{-1}$ progression in the upper state. This is a reasonable value for the highest frequency symmetric stretch. Peaks $A_{2}$ and $A_{5}$ appear to form $300 \mathrm{~cm}^{-1}$ progressions off of Peaks $A_{1}$ and $A_{3} / A_{4}$, respectively, which gives a $600 \mathrm{~cm}^{-1}$ frequency for the upper state. This is close to the predicted lowest-frequency symmetric stretch of neutral $C_{6}$, although the ZEKE spectrum yielded $480 \mathrm{~cm}^{-1}$ for the this mode in the ground neutral state.

$\mathbf{C}_{\mathrm{g}}$ - cross section: The structure observed well below the detachment continuum of $\mathrm{C}_{9}$ [botiom panel, Figure (4.3)], as with $\mathrm{C}_{6}$, appears to be transitions of type (d) depicted in Figure (4.4). Two-color experiments were also performed for $\mathrm{C}_{8}{ }^{-}$as they were for $\mathrm{C}_{6}{ }^{-}$, which further support this characterization. Based on this, the upper state lies $2.28 \mathrm{eV}$ above the detachment continuum. This, coincidentally, is identical to the energy of the $C_{6}^{*}$ upper state above the $C_{6}-$ detachment continuum. Peaks $A_{1}, A_{2}$ and $A_{3}$ form a $500 \mathrm{~cm}^{-1}$ progression, which gives a $1000 \mathrm{~cm}^{-1}$ frequency for the upper state. Peaks $A_{5}, A_{6}$ and $A_{7}$ form another $500 \mathrm{~cm}^{-1}$ progression with a different profile, and the two progressions may correspond to different electronic transitions.

\section{Conclusions}

The work presented here, if nothing else, illustrates how interesting the electronic properties of small carbon cluster anions are. The narrow and broad features observed in the total photodetachment cross sections of $\mathrm{C}_{4}^{-}, \mathrm{C}_{6^{-}}, \mathrm{C}_{7}, \mathrm{C}_{8}$ and $\mathrm{C}_{9}$ - presented here will hopefully inspire future investigations into these elusive species. 


\section{Acknowledgments}

This research was supported by the National Science Foundation under Grant No. CHE-8857636. 
1C. C. Amold, Y. Zhao, T. N. Kitsopoulos, and D. M. Neumark, J. Chem. Phys. 97, 6121 (1992).

2P. L. Jones, R. D. Mead, B. E. Kohler, S. D. Rosner, and W. C. Lineberger, J. Chem. Phys. 73, 4419 (1980); P. A. Schulz, R. D. Mead, P. L. Jones, and W. C. Lineberger, J. Chem. Phys. 77, 1153 (1982); U. Hefter, R. D. Mead, P. A. Schulz, and W. C. Lineberger, Phys. Rev. A 28, 1429 (1983).

3E. A. Brinkman, E. Günther, and J. I. Brauman, J. Chem. Phys. 95, 6185 (1991).

${ }^{4}$ D. M. Neidmark, K. R. Lykke, T. Andersen, and W. C. Lineberger, J. Chem. Phys. 83, 4364 (1985).

5K. R. Lykke, R. D. Mead, and W. C. Lineberger, Phys. Rev. Lett. 52, 2221 (1984).

${ }^{6}$ T. Anderson, K. R. Lykke, D. M. Neumark, and W. C. Lineberger, J. Chem. Phys. 86, 1858 (1987).

${ }^{7}$ K. R. Lykke, K. K. Murray, D. M. Neumark, and W. C. Lineberger, Phil. Trans. R. Soc. Lond. A 324, 179 (1988).

8K. J. Taylor, C. Jin, J. Conceicao, L-S. Wang, O. Cheshnovsky, B. R. Johnson. P. J. Nordlander, and R. E. Smalley, J. Chem. Phys. 93, 7515 (1990).

9V. Parasuk and J. Almöf, J. Chem. Phys. 91,1137 (1989); K. Raghavachani, R. A. Whiteside, and J. A. Pople, J. Chem. Phys. 85, 6623 (1986).

10T. N. Kitsopoulos, C. J. Chick, Y. Zhao, and D. M. Neumark, J. Chem. Phys. 95.5479 (1991).

'1D. W. Amold, S. E. Bradforth, T. N. Kitsopoulos, and D. M. Neumark, J. Chem. Phys. 95, 8753 (1991). 
12T. G. Dietz, M. A. Duncan, D. E. Powers, and R. E. Smalley, J. Chem. Phys. 74, 6511 (1981). 
Table (4.1) Electronic configurations and electron affinities of the linear cabon clusters and configurations of the corresponding anions.

\begin{tabular}{|c|c|c|c|c|c|}
\hline & $\begin{array}{l}\text { Neutral } \\
\text { State }\end{array}$ & $\begin{array}{l}\text { Electronic } \\
\text { configuration }\end{array}$ & $\begin{array}{l}\text { Electron } \\
\text { Affinity } \\
(\mathrm{eV})\end{array}$ & $\begin{array}{l}\text { Anion } \\
\text { ground } \\
\text { state }\end{array}$ & $\begin{array}{l}\text { Anion } \\
\text { configuration }\end{array}$ \\
\hline$c_{2}$ & $1 \Sigma_{g}+$ & $\ldots\left(2 \sigma_{g}\right)^{2}\left(2 \sigma_{u}\right)^{2}\left(1 \pi_{u}\right)^{4}$ & 3.273 & $2 \Sigma_{g}+$ & $\ldots\left(3 \sigma_{g}\right)^{t}$ \\
\hline$c_{3}$ & ${ }^{1} \Sigma_{g}+$ & $\ldots\left(4 \sigma_{g}\right)^{2}\left(3 \sigma_{u}\right)^{2}\left(1 \pi_{u}\right)^{4}$ & 1.995 & ${ }^{2} \Pi_{9}$ & $\ldots\left(1 \pi_{g}\right)^{1}$ \\
\hline $\mathrm{C}_{4}$ & ${ }^{3} \Sigma_{g}$ & $\begin{array}{l}\ldots\left(1 \pi_{u}\right)^{4}\left(4 \sigma_{u}\right)^{2}\left(5 \sigma_{g}\right)^{2} \\
\left(1 \pi_{g}\right)^{2}\end{array}$ & 3.882 & ${ }^{2}\left[I_{g}\right.$ & $\ldots\left(1 \pi_{g}\right)^{3}$ \\
\hline $\mathrm{C}_{5}$ & ${ }^{1} \Sigma_{g}+$ & $\underset{\left(1 \pi_{g}\right)^{4}}{\ldots\left(1 \pi_{u}\right)^{4}\left(6 \sigma_{g}\right)^{2}\left(5 \sigma_{u}\right)^{2}}$ & 2.855 & ${ }^{2} \Pi_{u}$ & $\ldots\left(2 \pi_{\mathrm{y}}\right)^{1}$ \\
\hline$c_{6}$ & ${ }^{3} \Sigma_{g}$ & $\begin{array}{l}\ldots\left(1 \pi_{u}\right)^{4}\left(6 \sigma_{u}\right)^{2}\left(7 \sigma_{g}\right)^{2} \\
\left(1 \pi_{g}\right)^{4}\left(2 \pi_{u}\right)^{2}\end{array}$ & 4.180 & ${ }^{2} \Pi_{u}$ & $\ldots\left(2 \pi_{4}\right)^{3}$ \\
\hline$c_{7}$ & ${ }^{1} \Sigma_{g}+$ & $\begin{array}{l}\ldots\left(1 \pi_{u}\right)^{4}\left(1 \pi_{g}\right)^{4}\left(8 \sigma_{g}\right)^{2} \\
\left(7 \sigma_{u}\right)^{2}\left(2 \pi_{v}\right)^{4}\end{array}$ & 3.358 & ${ }^{2} \Pi_{9}$ & $\ldots\left(2 \pi_{g}\right)^{1}$ \\
\hline $\mathrm{C}_{\mathrm{B}}$ & ${ }^{3} \Sigma_{g}$ & $\begin{array}{l}\ldots\left(1 \pi_{v}\right)^{4}\left(1 \pi_{g}\right)^{4}\left(8 \sigma_{u}\right)^{2} \\
\left(9 \sigma_{g}\right)^{2}\left(2 \pi_{\nu}\right)^{4}\left(2 \pi_{g}\right)^{2}\end{array}$ & 4.370 & ${ }^{2} \Pi_{g}$ & $\ldots\left(2 \pi_{g}\right)^{3}$ \\
\hline $\mathrm{C}_{9}$ & ${ }^{1} \Sigma_{g}+$ & $\begin{array}{l}\ldots\left(1 \pi_{u}\right)^{4}\left(1 \pi_{g}\right)^{4}\left(2 \pi_{\nu}\right)^{4} \\
\left(10 \sigma_{u}\right)^{2}\left(11 \sigma_{g}\right)^{2}\left(2 \pi_{g}\right)^{4} \\
\left(3 \pi_{u}\right)^{2}\end{array}$ & 3.684 & ${ }^{2} \Pi_{u}$ & $\ldots\left(3 \pi_{u}\right)^{3}$ \\
\hline
\end{tabular}


Table (4.2) Peak positions and relative energies for the $\mathrm{C}_{7}^{-}$total photodetachment sross section spectrum [Figure (4.1), top panel].

$\begin{array}{lll}\text { Peak } & \begin{array}{l}\text { Position } \\ (\mathrm{nm})\end{array} & \begin{array}{l}\text { Relative energy } \\ \left(\mathrm{cm}^{-1}\right)\end{array} \\ \mathrm{B} & 372.8 & -180 \\ \mathrm{~A}_{1} & 370.70 & -25 \\ & 370.35 & 0 \\ \mathrm{~A}_{2} & 367.38 & 218 \\ & 367.04 & 244\end{array}$

Table (4.3) Peak positions and relative energies in the $\mathrm{C}_{9}{ }^{-}$total photodetachment cross section spectnum [Figure (4.1), bottom panel].

$\begin{array}{lll}\text { Peak } & \begin{array}{l}\text { Position } \\ (\mathrm{nm})\end{array} & \begin{array}{l}\text { Relative energy } \\ \left(\mathrm{cm}^{-1}\right)\end{array} \\ \mathrm{B}_{1} & 375.2 & 0 \\ & 374.0 & 86 \\ \mathrm{~B}_{2} & 369.35 & 422 \\ \mathrm{~B}_{3} & 368.30 & 499 \\ \mathrm{~A}_{1} & 363.6 & 850 \\ \mathrm{~A}_{2} & 340.45 & 0 \\ \mathrm{~A}_{3} & 337.55 & 252 \\ \end{array}$


Table (4.4) Peak positions and relative energies for the $\mathrm{C}_{4}{ }^{-}$total photodetachment cross section spectrum [Figure (4.2), upper panel].

$\begin{array}{lll}\text { Peak } & \begin{array}{l}\text { Position } \\ (\mathrm{nm})\end{array} & \begin{array}{l}\text { Relative energy } \\ \left(\mathrm{cm}^{-1}\right)\end{array} \\ \mathrm{A}_{1} & 302.8 & 0 \\ \mathrm{~A}_{2} & 291.55 & 1274 \\ & 290.0 & 1458 \\ & 289.5 & 1517 \\ \mathrm{~A}_{3} & 284.9 & 2075 \\ & 283.96 & 2191 \\ & 282.68 & 2351 \\ & 280.7 & 2600 \\ & 279.8 & 2715 \\ A_{4} & 279.3 & 2779 \\ & 278.0 & 2946 \\ & 276.95 & 3082 \\ A_{5} & 275.4 & 3286 \\ A_{6} & 271.2 & 3848 \\ & 270.2 & 3985 \\ & 269.4 & 4094\end{array}$


Table (4.5) Positions and relative energies of broad structure observed in the total photodetachment cross section spectrum of $\mathrm{C}_{6}^{-}$[Figure (4.2), lowe: panel and Figure (4.3) upper panel].

Peak Position (nm)

Above detachment continuum

$A_{1}$

$A_{2}$

T

296

292

289.1

288.5

287.95

Below detachment continuum

$a_{2}$

$a_{1}$

$A_{1}$

$\mathrm{A}_{2}$

$A_{3}$

$\mathrm{A}_{4}$

$A_{5}$

$A_{6}$

$A_{7}$

392.4

389.0

384.9

383.7

379.8

371.55

369.5

366.1

360.5

358.0
Relative Energy

$\left\{\mathrm{cm}^{-1}\right\}$

0

462

0

72

138

$-497$

$-274$

0

81

349

934

1083

1334

1759

1952 
Table (4.6) Peak positions and relative energies for the $\mathrm{C}_{8}{ }^{-}$total photodetachment cross section spea in [Figure (4.3), lower panel].

\begin{tabular}{|c|c|c|}
\hline Peak & $\begin{array}{l}\text { Position } \\
\text { (nm) }\end{array}$ & $\begin{array}{l}\text { Relative Energy } \\
\left(\mathrm{cm}^{-1}\right)\end{array}$ \\
\hline$a_{2}$ & 378.0 & -370 \\
\hline$a_{1}$ & $\begin{array}{l}376.2 \\
375.6\end{array}$ & $\begin{array}{l}-243 \\
-201\end{array}$ \\
\hline$A_{1}$ & $\begin{array}{l}373.4 \\
372.79\end{array}$ & $\begin{array}{l}-44 \\
0\end{array}$ \\
\hline$A_{2}$ & $\begin{array}{l}366.6 \\
366.01 \\
365.0 \\
364.2\end{array}$ & $\begin{array}{l}453 \\
497 \\
573 \\
633\end{array}$ \\
\hline$A_{3}$ & 358.5 & 1069 \\
\hline$A_{4}$ & 355.2 & 1485 \\
\hline$A_{5}$ & $\begin{array}{l}352.88 \\
352.24 \\
351.6\end{array}$ & $\begin{array}{l}1514 \\
1565 \\
1617\end{array}$ \\
\hline$A_{6}$ & $\begin{array}{l}347.2 \\
346.7 \\
345.9\end{array}$ & $\begin{array}{l}1977 \\
2019 \\
2085\end{array}$ \\
\hline$A_{7}$ & $\begin{array}{l}342.45 \\
341.45 \\
340.6 \\
340.2 \\
339.67\end{array}$ & $\begin{array}{l}2377 \\
2462 \\
2535 \\
2570 \\
2616\end{array}$ \\
\hline
\end{tabular}


Figure (4.1) Tolal photodetachment cross section spectra of $\mathrm{C}_{7}{ }^{-}$and $\mathrm{C}_{9}{ }^{-}$(solid lines) and the corresponding anion PES (dashed line).
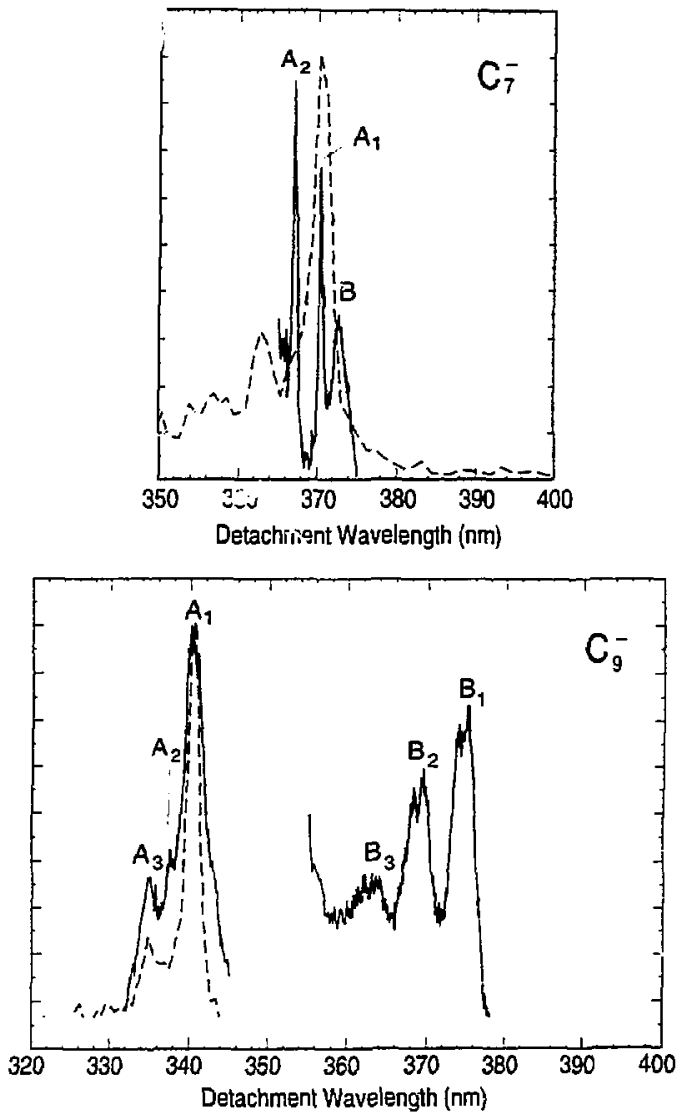
Figure (4.2) Total photodetachment cross section spectra of $\mathrm{C}_{4}{ }^{-}$and $\mathrm{C}_{6}{ }^{-}$above the respective detachment continua (solid lines) and the corresponding PES (dashed line).
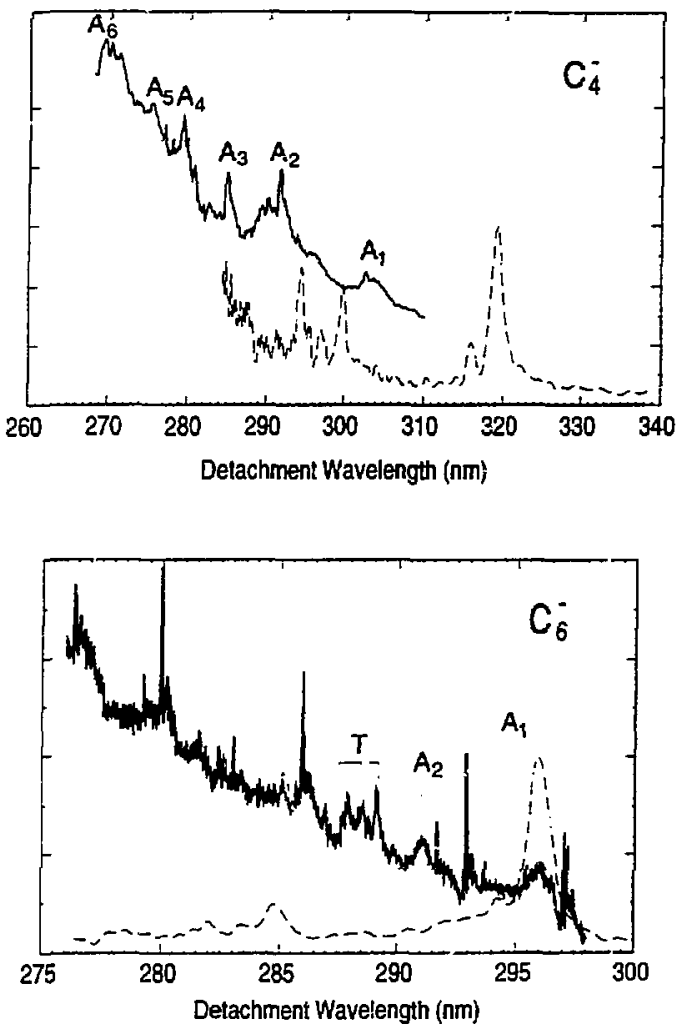
Figure (4.3) Total photodefachment cross section spectra of $\mathrm{C}_{6}{ }^{-}$and $\mathrm{C}_{8}{ }^{-}$below the respective detachment continua (solid lines) and the corresponding PES (dashed line).

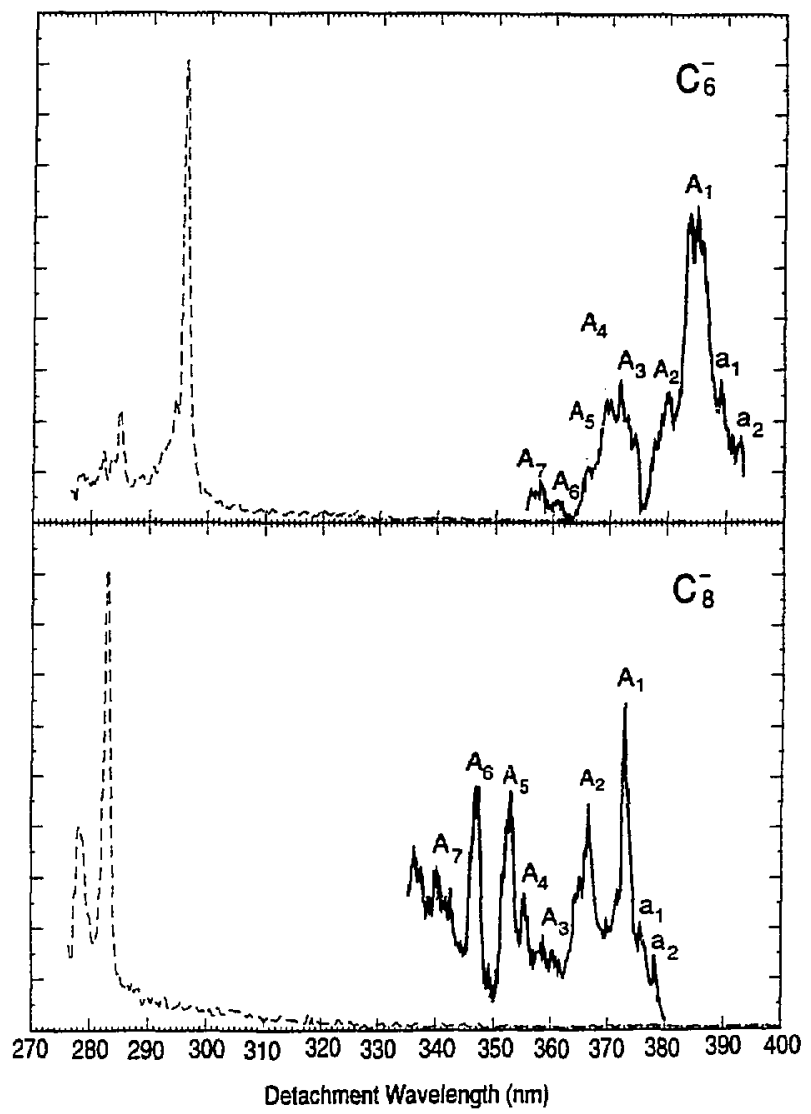


Figure (4.4) Possible transitions for the structure observed in the total photodetachment cross sections of small carbon clusters.

(a)

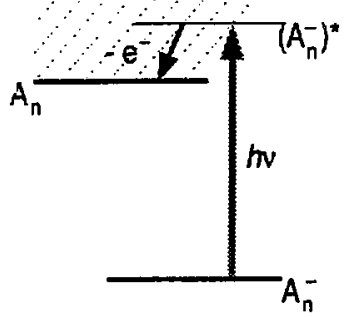

(c)

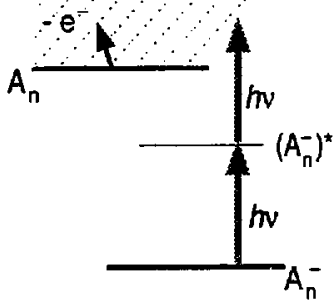

(b)

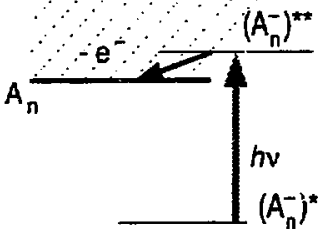

$A_{n}^{-}$

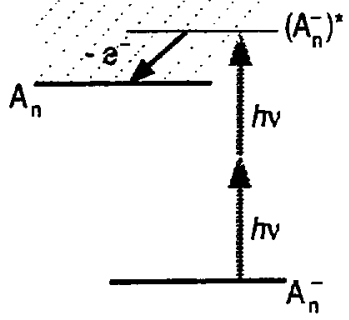




\section{Chapter 5. Reassignment of the $\mathrm{Si}_{2}$ - photodetachment spectra}

Upon reanalysis of our silicon cluster data, we have discovered that our analysis of our $\mathrm{Si}_{2}-$ photoelectron and threshold photodetachment spectra reported previously is incorrect. The mistake lies in improperly correlating peaks seen in the photoelectron spectrum (PES) with those found in the threshold photodetachment (or zero electron kinetic energy - ZEKE) spectrum. Figure (5.1) shows the PES of $\mathrm{Si}_{2}-$ with the detachment laser wavelength of $355 \mathrm{~nm}$ $(3.493 \mathrm{eV})$ and polarization $90^{\circ}$ with respect to the electron detection. Figure (5.2) shows the ZEKE spectrum of $\mathrm{Si}_{2}-$ corresponding to the $1.1-1.4 \mathrm{eV}$ region in the $3.49 \mathrm{eV}$ PES. A more recently obtained, higher resolution PES (dotted line) taken using $2.98 \mathrm{eV}$ photon energy has been superimposed onto the ZEKE spectrum (solid line) in Figure (5.2). Originally, we believed that peaks " $d$ " and " $C$ " in the PES corresponded, respectively, to the triplet feature at $555 \mathrm{~nm}$ and the doublet feature found at $570 \mathrm{~nm}$ in the ZEKE spectrum. However, a more careful calibration indicates that the triplet corresponds to peak D in PES, and that peak $d$ is absent in the ZFKE spectrum. The energy interval between $570 \mathrm{~nm}$ and $555 \mathrm{~nm}$ is $532 \mathrm{~cm}^{-1}$. Peaks "d" an: " $\mathrm{C}$ " on the PES are separated by oniy $350 \mathrm{~cm}^{-1}$, while "D* and "C" are separated by 550 $\mathrm{cm}^{-7}$. The ZEKE spectrum shown in Figure (5.2) has been relabelled to correctly refiect the correspondence with the PES peaks. 
This change affects the relative energies of the low-lying states of $\mathrm{Si}_{2}$ and $\mathrm{Si}_{2}-$, which are displayed in Figure (5.3). Peaks A through $F$ are assigned to transitions between two nearly Jegenerate doublet states of the anion and the two neaby triplet states of the neutral. The assignment of the ZEKE spectrum is the same as before: the triplets (peaks D,E) are due to ${ }^{3} \Pi_{u} \leftarrow{ }^{2} \Sigma_{g}+$ transitions, and the doublets (peaks B,C) are from ${ }^{3} \Sigma_{g}-{ }^{2} \Pi_{u}$ transitions. (The triplet and doublet structure is from the spin-orbit fine structure in the ${ }^{3} n_{u}$ and ${ }^{2} \Pi_{u}$ states, respectively). However, the PES assignment is now altered: peak $D$ is the ${ }^{3} \Pi_{u} \leftarrow{ }^{2} \Sigma_{g}+$ transition, while peak $d$ is the ${ }^{3} \Pi_{u} \leftarrow{ }^{2} \Pi_{u}$ transition; this assignment was reversed in our previous paper. Peak $d$ is observed in the PES but not the ZEKE spectrum because it is a pwave transition involving ejection of a photoelectron with orbital angular momentum $\ell=1$; the threshold photodetachment spectrometer is sensitive only to s-wave $(\ell=0)$ photodetachment transitions, since, according to the Wigner threshold law, ${ }^{2}$ these are the only transitions with substantial cross sections near the detachment threshold.

The implications of this reassignment are as follows. Since peak D corresponds to a higher energy transition than peak $d$, the ${ }^{2} \Sigma_{g}{ }^{+}$state of the anion must lie below, rather than above, the ${ }^{2} \Pi_{U}$ state. This means that the eriergy of peak $C$ no longer corresponds to the electron affinity of $\mathrm{Si}_{2}$ because it is not the transition between the lowest electronic states of the anion and neutral. The "electron affinity" transition, from the anion ${ }^{2} \Sigma_{g}+$ state to the neutral ${ }^{3} \Sigma_{g}$ state, is a two-electron transition, involving both photodetachment and rearrangement of the remaining electrons. Such a transition is, in general, too weak to be observed in either the PES or ZEKE spectrum. We therefore do not see the electron affinity transition here, afthough the electron affinity can be extracted from the observed transitions.

A similar error was made in the singlet manifold, peaks G-L. The transitions are shown in Figure (5.3). Figure (5.4) shows the threshold photodetachment spectrum in the 0.6 to 0.95 $\mathrm{eV}$ energy region in the PES. The peak found at $451 \mathrm{~nm}$ was originally believed to correspond to peak 1 in the PES. However, with our improved PES data calibration, we now know that it 
corresponds to peak $J$ in the PES. The $451 \mathrm{~nm}$ peak was correctly assigned to the $b^{1} \Pi_{v} \leftarrow{ }^{2} \Sigma_{g}{ }^{+}$ transition. Peak $\mid$ in the PES is then assigned to the $b^{1} \Pi_{u} \leftarrow{ }^{2} \Pi_{u}$ transition, a p-wave transition that will not be observed in the ZEKE spectrum. The relative position of these two peaks is consistent with the ${ }^{2} \Sigma_{g}+$ state being the ground state of the anion.

Several of the peak labels on Figs. 5 and 6, and Table 1 in Ref. [1] should be changed in order to properly refiect the relationship between the PES and the threshold photodetachment spectrum. As mentioned before, "d $d_{1,2,3}$ " and " $e_{1,2,3}$ " in Figure 5, Ref. [1] should be changed to

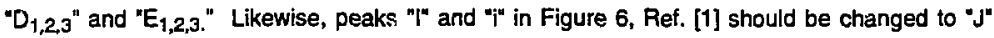
and "j." Figures (5.2) and (5.4) presented here have these new labels. Also indicated by vertical lines in Figures (5.2) and (5.4) are the positions of peaks $d$ and I taken from the newly calibrated PES data.

Table (5.1) lists these threshold spectral peaks by the new label along with the position and assignment. Additionally, the p-wave transitions observed only in the PES are included in Table (5.1) with the improved energy position. These are marked by an asterisk ("). Several of these peaks have different assignments than they were given in Ref. [1] as a result of the above discussion.

Table (5.2) summanizes the excitaiton energies of the anion and neutral states based on the the correct ordering of the anion states. ${ }^{3}$ The correct electron affinity of $\mathrm{Si}_{2}, 2.201 \pm 0.01$ $\mathrm{eV}$, is also given. Vibrational frequencies and spin-orbit splittings remain unchanged for all of the states. Note that $r_{e}$ for the ${ }^{3} \Pi_{v}$ state is $2.155 \dot{A}_{i}^{* 4}$ a typographical error was made in Ref.[1]. Interestingly, calculations pertormed on the anion by Bruna et al., ${ }^{5}$ Nimlos et. al. ${ }^{6}$ and Raghavachari et. al. ${ }^{7}$ predict the ${ }^{2} \Sigma_{g}{ }^{+}$state to be the ground state. In fact, the highest level calculation performed by Raghavachari predicted the $\Pi$ state to lie $22 \mathrm{meV}$ above the $\Sigma$ state, which is remarkably close to our experimental value of $25 \mathrm{meV}$. It should also be noted that Weltner has recently corroborated this reassignment with IR spectra of neutral $\mathrm{Si}_{2}$ in rare gas 
matrices..$^{8}$ From his spectra, he found the ${ }^{3} \Pi_{\mathrm{U}} \cdot{ }^{3} \Sigma_{\mathrm{g}} \cdot$ splitting to be $313 \mathrm{~cm}^{-1}$, which is well within the error bars of our value, $331 \pm 80 \mathrm{~cm}^{-1}$.

This research is supported by the National Science Foundation under Grant No. DMR-9201159.

1T. N. Kitsopoulos, C. J. Chick, Y. Zhao, and D. M. Neumark, J. Chem. Phys. 95, 1441 (1991).

2E. P. Wigner, Phys. Rev. 73, 1003 (1948).

${ }^{3}$ The photon energy of the detachment laser was such that transitions to the $d^{1} \Sigma$ were not observed, but IR emission between this state and the $b^{1} \Pi_{U}$ state was reported by S. P. Davis and J. W. Brault, J. Opt. Soc. Am. B 4, 20 (1987). The two states were determined to lie 0.608 eV apart.

${ }^{4}$ A. E. Douglas, Can. J. Phys. 33, 801 (1955).

5P. J. Bruna, H. Dohmann, J. Anglada, V. Krumbach, S. D. Peyerimhoff, and R. J. Buenker, J. Mol. Struct. 93, 309 (1983).

${ }^{6}$ M. R. Nimlos, L. B. Harding, and G. B. Ellison, J. Chem. Phys. 87, 5116 (1987).

7K. Raghavachari and C. M. Rohising, J. Citem. Phys. 94, 3670 (1991).

6S. Li, R. J. Van Zee, and W. Weltner, Jr., J. Chem. Phys. 100, 7079 (1994). 
Table (5.1) Peak positions and assignments for the ZEKE spectrum of $\mathrm{Si}_{2}{ }^{-}$. Peaks marked with an asterisk were not seen on the ZEKE spectrum, and are taken from the PES spectrum.

\begin{tabular}{|c|c|c|}
\hline Peak & $\begin{array}{l}\text { Position } \\
\text { (eV) }\end{array}$ & Assignment \\
\hline $\mathrm{B}_{2}$ & 2.095 & $x^{3} \Sigma_{0}\left(v^{\prime}=0\right) \leftarrow{ }^{2} \Pi_{1 / 2}\left(v^{\prime \prime}=1\right)$ \\
\hline $\mathrm{B}_{1}$ & 2.109 & $x^{3} \Sigma_{0}-\left(v^{\prime}=0\right) \leftarrow{ }^{2} \Pi_{3 / 2}\left(v^{N}=1\right)$ \\
\hline $\mathrm{C}_{2}$ & 2.150 & $x^{3} \Sigma_{\mathrm{a}}{ }^{-}\left(v^{\prime}=0\right) \leftarrow{ }^{2} \Pi_{1 / 2}\left(v^{\prime \prime}=0\right)$ \\
\hline c & 2.165 & $D^{3} \Pi_{2}\left(v^{\prime}=0\right) \leftarrow{ }^{2} \Sigma_{a}+\left(v^{\prime \prime}=1\right)$ \\
\hline$c_{1}$ & 2.176 & $x^{3} \Sigma_{0}-\left(v^{\prime}=0\right) \leftarrow{ }^{2} \Pi_{3 / 2}\left(v^{\prime \prime}=0\right)$ \\
\hline c & 2.183 & $D^{3} \Pi_{0}\left(v^{\prime}=0\right) \leftarrow{ }^{2} \Sigma_{a}+\left(i^{\prime \prime}=1\right)$ \\
\hline$d^{*}$ & 2.222 & $D^{3} \Pi_{u}\left(v^{\prime}=0\right) \leftarrow{ }^{2} \Pi_{u}\left(v^{\prime \prime}=0\right)$ \\
\hline$c_{2}$ & 2.225 & $x^{3} \Sigma_{0}\left(v^{\prime}=1\right) \leftarrow{ }^{2} \Pi_{1 / 2}\left(v^{\prime \prime}=0\right)$ \\
\hline$D_{1}$ & 2.235 & $D^{3} \Pi_{2}\left(v^{\prime}=0\right) \leftarrow{ }^{2} \Sigma_{a}+\left(v^{\prime \prime}=0\right)$ \\
\hline$D_{2}$ & 2.242 & $D^{3} \Pi_{1}\left(v^{\prime}=0\right) \leftarrow{ }^{2} \Sigma_{a}+\left(v^{\prime \prime}=0\right)$ \\
\hline$D_{3}$ & 2.250 & $D^{3} \Pi_{0}\left(v^{\prime}=0\right) \leftarrow{ }^{2} \Sigma_{a}+\left(v^{\prime \prime}=0\right)$ \\
\hline$e^{*}$ & 2.287 & $D^{3} \Pi_{u}\left(v^{\prime}=1\right) \leftarrow{ }^{2} \Pi_{u}\left(v^{\prime \prime}=0\right)$ \\
\hline$E_{1}$ & 2.301 & $D^{3} \Pi_{2}\left(v^{\prime}=1\right) \leftarrow{ }^{2} \Sigma_{a}+\left(v^{\prime \prime}=0\right)$ \\
\hline$E_{2}$ & 2.309 & $D^{3} \Pi_{1}\left(v^{\prime}=1\right) \leftarrow{ }^{2} \Sigma_{a}+\left(v^{\prime \prime}=0\right)$ \\
\hline$E_{3}$ & 2.316 & $D^{3} \Pi_{0}\left(v^{\prime}=1\right) \leftarrow{ }^{2} \Sigma_{a}+\left(v^{\prime \prime}=0\right)$ \\
\hline$F^{*}$ & 2.326 & $?$ \\
\hline $\mathbf{G}_{1}$ & 2.597 & $a^{1} \Delta_{\sigma}\left(v^{\prime}=0\right) \leftarrow{ }^{2} \Pi_{1 / 2}\left(v^{\prime \prime}=0\right)$ \\
\hline $\mathrm{G}_{2}$ & 2.611 & $a^{1} \Delta_{d}\left(v^{\prime}=0\right) \leftarrow{ }^{2} \Pi_{3 / 2}\left(v^{\prime \prime}=0\right)$ \\
\hline $\mathrm{H}_{1}$ & 2.657 & $a^{1} \Delta_{o}\left(v^{\prime}=1\right) \leftarrow{ }^{2} \Pi_{1 / 2}\left(v^{\prime \prime}=0\right)$ \\
\hline $\mathrm{H}_{2}$ & 2.672 & $a^{1} \Delta_{a}\left(v^{\prime}=1\right) \leftarrow{ }^{2} \Pi_{3 / 2}\left(v^{\prime \prime}=0\right)$ \\
\hline$M_{1}$ & 2.715 & $a^{9} \Delta_{0}\left(v^{\prime}=2\right) \leftarrow{ }^{2} \Pi_{1 / 2}\left(v^{\prime \prime}=0\right)$ \\
\hline$M_{2}$ & 2.729 & $a^{1} \Delta_{0}\left(v^{\prime}=2\right) \leftarrow{ }^{2} \Pi_{3 / 2}\left(v^{\prime \prime}=0\right)$ \\
\hline$I^{*}$ & 2.722 & $b^{\top} \Pi_{u}\left(v^{\prime}=\tilde{v}\right) \leftarrow{ }^{2} \Pi_{u}\left(v^{\prime \prime}=0\right)$ \\
\hline$J$ & 2.745 & $b^{1} \Pi_{u}\left(u^{\prime}=0\right) \leftarrow{ }^{2} \Sigma_{a}+\left(v^{\prime \prime}=0\right)$ \\
\hline $\mathbf{K}^{*}$ & 2.787 & $c^{1} \Sigma_{a}+(v=0) \leftarrow{ }^{2} \Sigma_{0}+\left(v^{\prime \prime}=0\right)$ \\
\hline j & 2.812 & $b^{1} \Pi_{u}\left(v^{\prime}=1\right) \leftarrow{ }^{2} \Sigma_{0}+\left(v^{\prime \prime}=0\right)$ \\
\hline$L *$ & 2.826 & $?$ \\
\hline
\end{tabular}


Table (5.2) Excitation energies for the low-lying states of $\mathrm{Si}_{2}$ and $\mathrm{Si}_{2}$ *

\begin{tabular}{|c|c|c|}
\hline & State & $\begin{array}{r}T_{\mathrm{e}} \\
(\mathrm{eV}) \\
\end{array}$ \\
\hline \multirow{6}{*}{$\begin{array}{c}\text { E.A. }= \\
2.201 \pm 0.010 \mathrm{eV}\end{array}$} & $d^{\prime} \Sigma_{0}{ }^{+}$ & $1.152 \pm 0.010$ \\
\hline & $c^{\prime} \Sigma_{0}+$ & $0.586 \pm 0.010$ \\
\hline & $b^{1} \Pi_{11}$ & $0.544 \pm 0.010$ \\
\hline & $a^{\top} \Delta_{a}$ & $0.435 \pm 0.002$ \\
\hline & $D^{3} \Pi_{11}$ & $0.041 \pm 0.010$ \\
\hline & $x^{3} \Sigma_{0}^{-}$ & 0 \\
\hline \multirow[t]{2}{*}{$\mathrm{Si}_{2}-$} & ${ }^{2} \Pi_{u}$ & $0.025 \pm 0.010$ \\
\hline & ${ }^{2} \Sigma_{-1}+$ & 0 \\
\hline
\end{tabular}




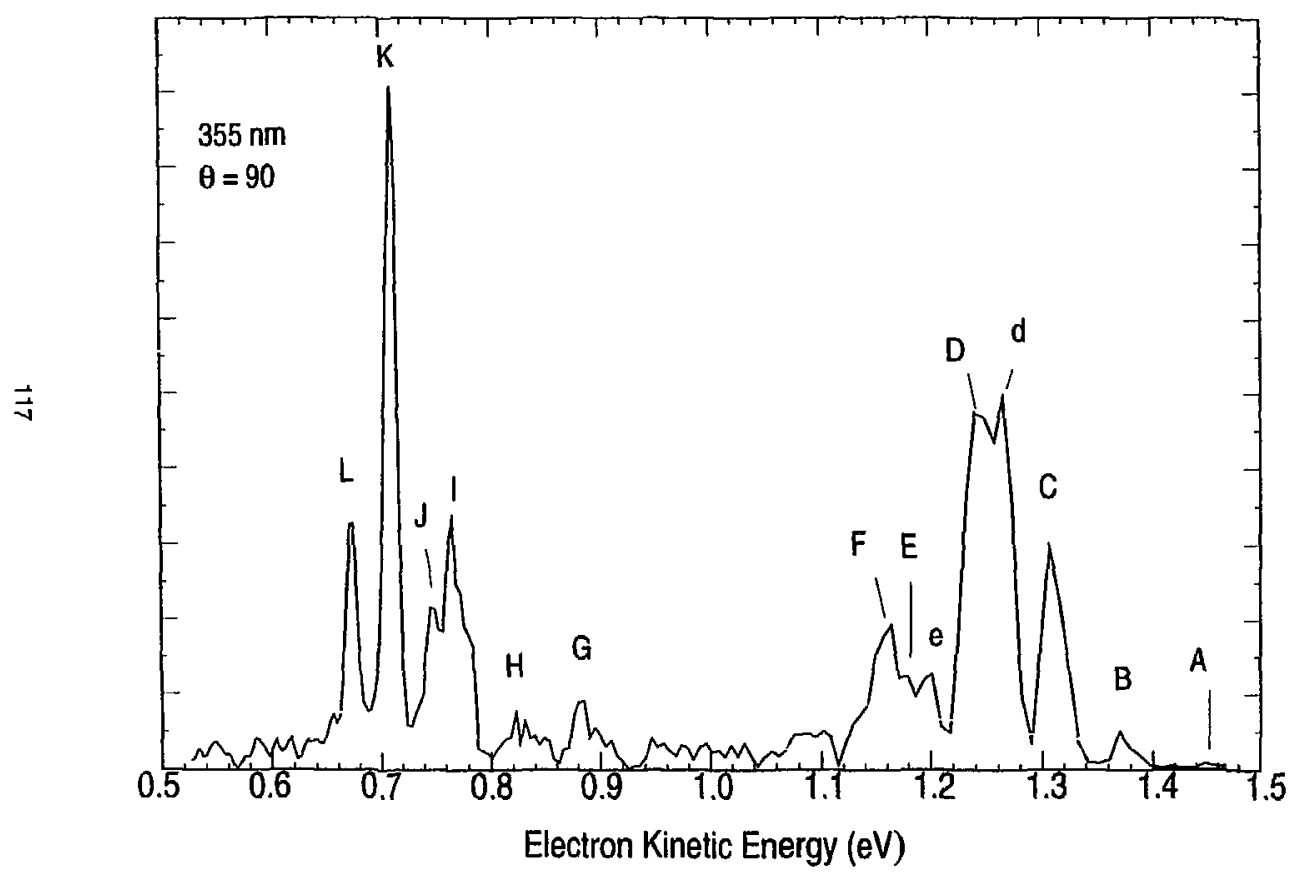

㿣

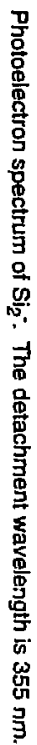


Figure (5.2) ZEKE spectrum of $\mathrm{Si}_{2}-$ showing transitions to the triplet states of $\mathrm{Si}_{2}$.

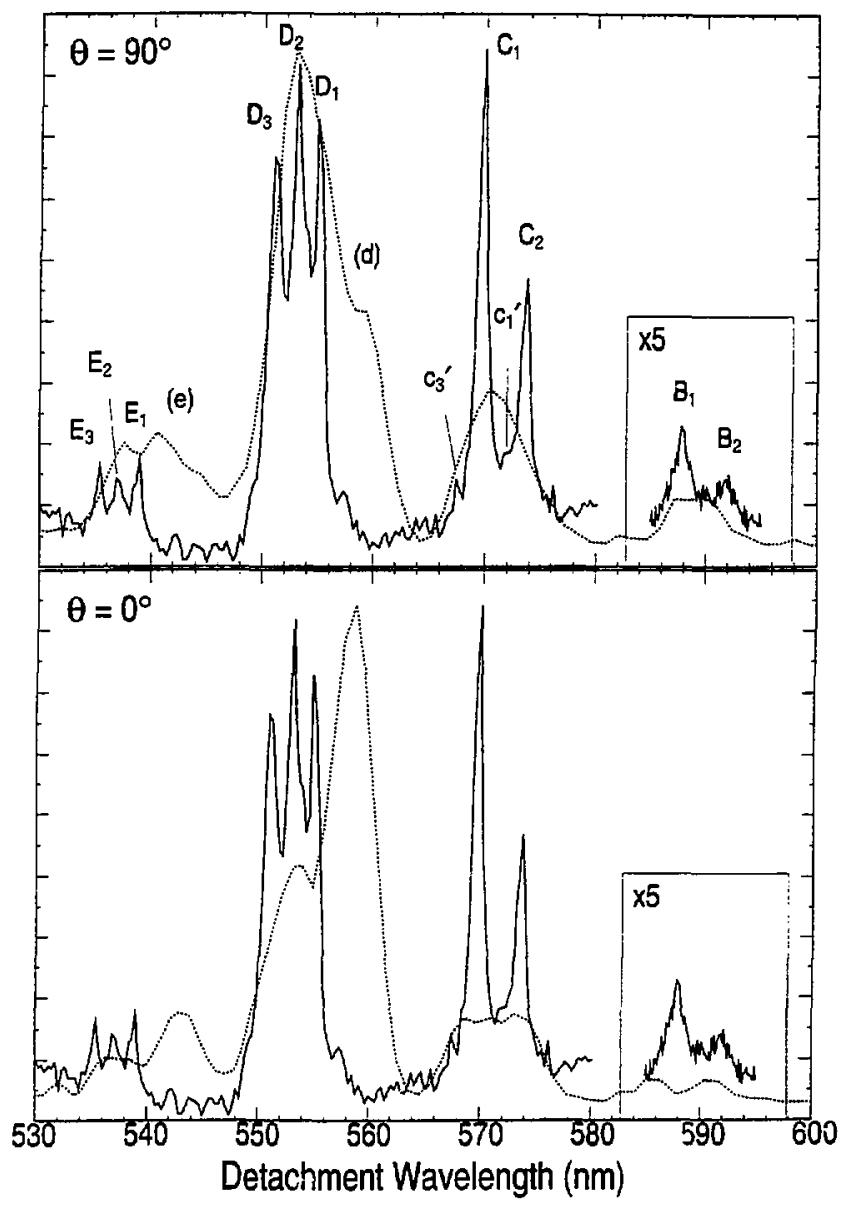


Figure (5.3) One-electron transitions between the low-lying states of $\mathrm{Si}_{2}-$ and $\mathrm{Si}_{2}$. Those marked with an asterisk are p-wave transitions. Multiple letters correspond to different vibrational transitions [see Table (5.1)].

$\mathrm{d}^{\mathrm{I}} \Sigma_{\mathrm{g}}^{+}$

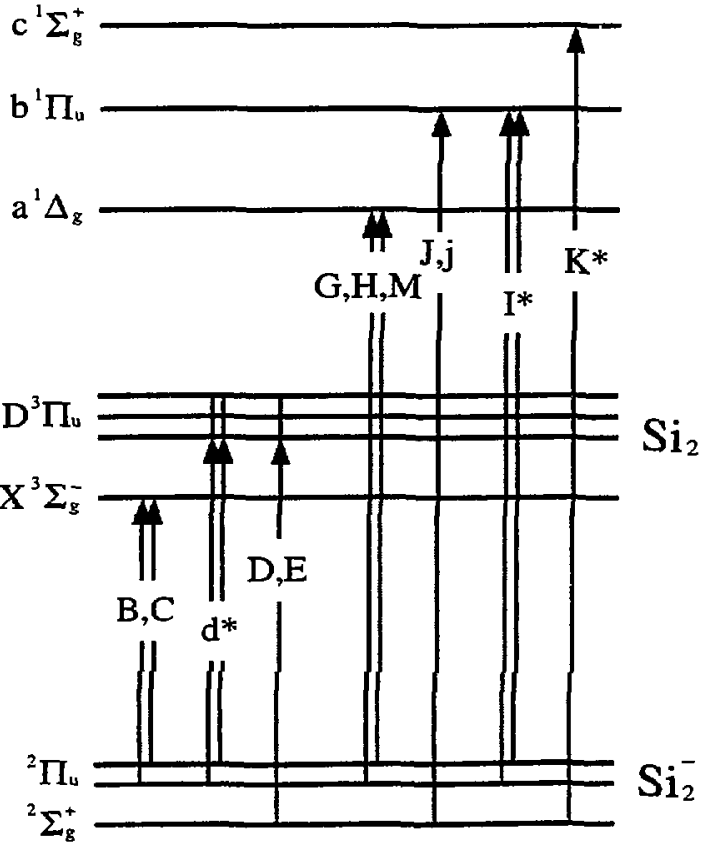


Figure (5.4) ZEKE spectrum of $\mathrm{Si}_{2}$ - showing transitions to the singlet states of $\mathrm{Si}_{2}$.

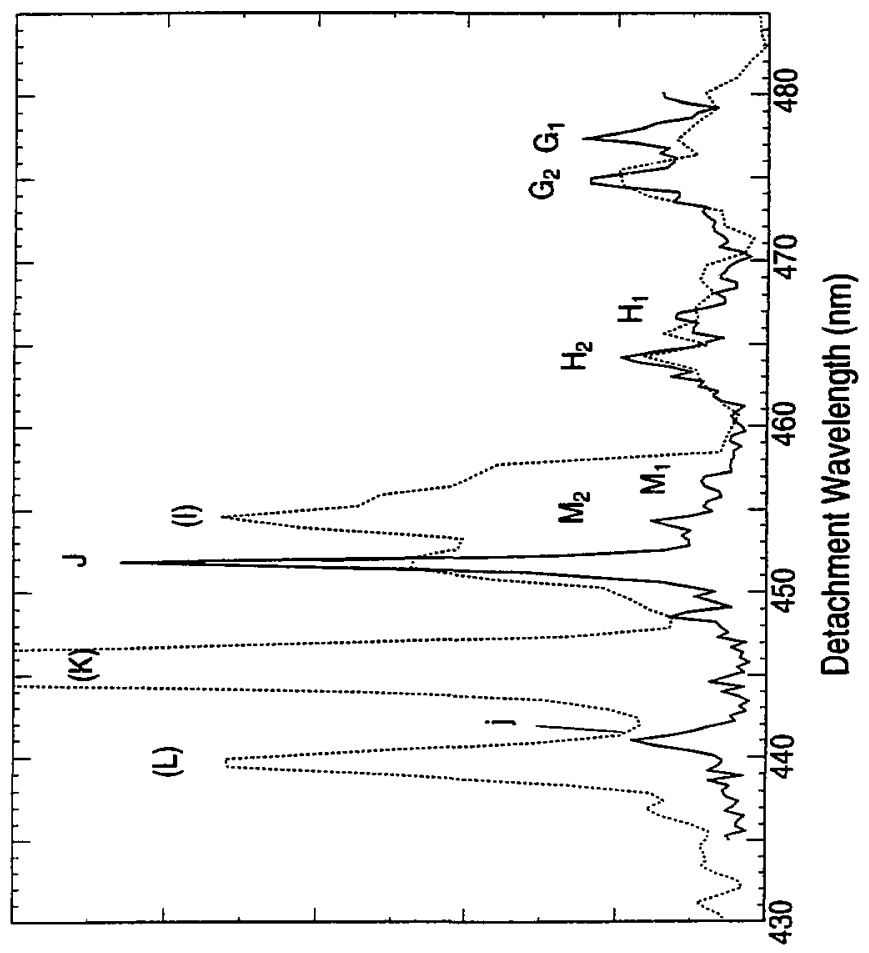




\section{Chapter 6. Threshold Photodetachment (ZEKE) Spectroscopy of $\mathrm{Si}_{3}=$}

\section{Abstract}

The threshold photodetachment (ZEKE) spectrum of $\mathrm{Si}_{3}{ }^{-}$is presented and discussed. The spectrum shows well-resolved vibrational structure. A comparison with several ab initio calculations shows that the spectrum is due to transitions to the ${ }^{3} \mathrm{~A}_{2}{ }^{\prime}\left(\mathrm{D}_{3 n}\right)$ state of $\mathrm{Si}_{3}$. The symmetric stretch and degenerate $e^{\prime}$ frequencies for the $\mathrm{Si}_{3}{ }^{3} \mathrm{~A}_{2}$ state are determined to be 501 $\pm 10 \mathrm{~cm}^{-1}$ and $337 \pm 10 \mathrm{~cm}^{-1}$, respectively. Additional structure observed in the spectrum suggests that the negative ion is a fluxional, Jahn-Teller distorted species. The ZEKE spectrum shows no evidence for transitions to the ${ }^{1} A_{1}$ state of the neutral, which is predicted to lie very close to the ${ }^{3} A_{2}$ state. A comparison of the ZEKE and previously obtained photoelectron spectrum of $\mathrm{Si}_{3}{ }^{-}$suggests that these two states are close in energy, but that transitions to the singlet state are very weak in the ZEKE spectrum. 


\section{Introduction}

The study of small silicon clusters has been motivated by their importance in

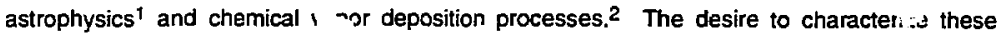
species experimentally has been further fueled by a series of ab initio studies that have predicted interesting electronic properties and structural trends as a function of cluster size. ${ }^{3}$ However, other than for the dimer, 4.5 experimental work on these elusive species is sparse; thus far, for example, vibrationally resolved spectra have been obtained only for $\mathrm{Si}_{3}$ and $\mathrm{Si}_{4}, 6,7,8$ and no rotationally resolved spectra have been observed for any species larger than the dimer. In our laboratory, we are focussing on probing the electronic and vibrational structure of these clusters by performing high resolution photodetachment spectroscopy experiments. This paper discusses the results of such a study performed on $\mathrm{Si}_{3}$.

Silicon trimer has been the object of considerable theoretical efforts, starting with the work by Grev and Schaefer ${ }^{9}$ and by Raghavachar ${ }^{10}$ in 1985 . The results of their calculations for an extensive set of molecular structures indicated that, in contrast with the well-known linear structure of $\mathrm{C}_{3}{ }^{11} \mathrm{Si}_{3}$ is strongly bent, with two close lying electronic states: the ${ }^{1} \mathrm{~A}_{1}\left(\mathrm{C}_{2 v}\right)$ and ${ }^{{ }^{3} A_{2}}\left(D_{3 h}\right)$ states. Soth calculations predict the singlet $C_{2 v}$ structure to be the ground state, but the calculated spacing between the two states is less than $1 \mathrm{kcal} / \mathrm{mol}$. More recent calculations have yielded similar results. ${ }^{12,13,14,15}$ The negative ion of $\mathrm{Si}_{3}$, while less studied, is also interesting from a theoretical standpoint. Rohlfing and Raghavachari ${ }^{16,17}$ repor:ed a ${ }^{2} A_{1}\left(C_{2 v}\right.$ geometry) ground state winich results from Jahn-Teller distortion of $a^{2} E^{\prime}$ configuration, with the corresponding ${ }^{2} \mathrm{~B}_{2}$ saddle point lying only $160 \mathrm{~cm}^{-1}$ higher in energy. Raghavachari ${ }^{10,16,17}$ has calculated vibrational frequencies for the anion as well as the low-lying neviral states, and frequencies for the neutral states have also been calculated by Dixon ${ }^{12}$ and Depristo. ${ }^{14}$ 
The experimental work to date on $\mathrm{Si}_{3} / \mathrm{Si}_{3}{ }^{-}$has not been of sufficient quality to test the $a b$ initio predictions conceming electronic and vibrational stnucture. The negative ion photoelectron spectnm (PES) of $\mathrm{Si}_{3}{ }^{-}$obtained by Smalley and coworkers ${ }^{18}$ reveajed several electronic bands, but the resolution was insufficient to make any assignments. In the vibrationally resolved PES of $\mathrm{Si}_{3}{ }^{-}$obtained by our group, ${ }^{6}$ we observed that the band associated with the lowest $\mathrm{Si}_{3}$ electronic state exhibited an extended vibrational progression with a characteristic frequency of $360 \pm 40 \mathrm{~cm}^{-1} \cdot$ in poor agreement with the calculated frequencies of the purported ground ${ }^{1} A_{1}$ state. However, this progression was congested and poorty resolved, and a better quality spectrum was clearly needed for comparison with the ab iritio work. A similar frequency was observed in emission spectra obtained by Gole and coworkers ${ }^{7}$ and attributed to $\mathrm{Si}_{3}$.

In this paper, the substantially higher resolution threshold photodetachment (zero kinetic energy electron, or ZEKE) spectrum of $\mathrm{Si}_{3}{ }^{-}$is presented. As with the ZEKE spectra we obtained for $\mathrm{Si}_{2}{ }^{-}$and $\mathrm{Si}_{4}{ }^{-5,8}$ more detailed information regarding both the $\mathrm{Si}_{3}$ anion and neutral electronic states is extracted from the spectrum presented below than could be determined from the PES. The spectrum, which could be obtained over only a small part of the energy range probed by the PES, shows well-resolved transitions between what appear to be the ground electronic states of the anion and neutral trimer. This spectral structure, however, is still not reconcilable with the predicted ${ }^{2} \mathrm{~A}$, anion and ${ }^{1} \mathrm{~A}$, neutral ground state frequencies. ${ }^{10.12 .14 .17}$ instead, it appears that the band is due to transitions to the neutral ${ }^{3} \mathrm{~A}_{2} \cdot\left(\mathrm{D}_{3 n}\right)$ state which, as mentioned above, is predicted to be nearly degenerate with, but slightly higher in energy than the calculated neutral ground ${ }^{1} \mathrm{~A}_{1}$ state. The ZEKE spectrum also exhibits additional structure not resolved in the PES. In order to satisfactorily assign this structure, it is necessary to treat the anion as a fluxional, Jahn-Teller disto.ted species rather than as a rigid molecule. 


\section{Il. Experimental}

The threshold photodetachment spectrometer used in this study is described in detail elsewhere, ${ }^{19}$ but the basic operation is as follows. A beam of cold silicon cluster anions is generated in a laser vaponzation/pulsed molecular beam source and is mass-selected using time-of-ilight. The cluster of interest is then photodetached using an excimer-pumped dye laser operated at $20 \mathrm{~Hz}$. The electrons detached with nearly zero kinetic energy (ZEKE) are collected as a function of photon wavelength. The selective detection of ZEKE electrons, based on the method developed by Müller-Dethlefs et al., ${ }^{20}$ gives an energy resolution of as good as $3 \mathrm{~cm}^{-1}$. However, because of the small photodetachment cross section of $\mathrm{Si}_{3}{ }^{\circ}$, some resolution was sacrificed for increased signal; the resolution for the spectrum presented below is estimated to be 10 to $15 \mathrm{~cm}^{-1}$. The signal was averaged for 1500 shots at each wavelength and normalized to both laser power and ion current. The two dyes used to obtain the spectrum were Coumarin 540 and Cotmanin 503.

\section{Results}

Figure (6.1) shows the PES of $\mathrm{Si}_{3}$ previously obtained with a detachment wavelength of $355 \mathrm{~nm}(3.493 \mathrm{eV}){ }^{6}$ Band $\mathrm{X}$ represents transitions to the lowest electronic state(s) of $\mathrm{Si}_{3}$, while band $B$ is a transition to a more highly excited state. The inset shows band $X$ on an expanded $y$-axis (dotted line) with the newly obtained ZEKE spectrum superimposed onto the PES energy scale. We observed a ZEKE spectrum for this band only. The data collection was considerably more time consuming than in our recent study of $\mathrm{Si}_{4}-{ }^{-8}$ the photodetachment cross section near threshold was much smaller for $\mathrm{Si}_{3}{ }^{-}$than for $\mathrm{Si}_{4}$, and the trimer ions were more difficult to produce. Figure (6.2) shows the ZEKE spectrum of band $X$ on an expanded scale showing the detachment wavelength. The spectrum is dominated by a $337 \mathrm{~cm}^{-1}$ progression formed by the 
$18-20 \mathrm{~cm}^{-1}$ wide (FWHM) peaks labeled $0,1,2,3$, and 4. Peak $\sigma^{\prime}$ is not a member of this progression; it lies $385 \mathrm{~cm}^{-1}$ to lower photon energy of peak 0 . which appears to be the origin of the main progression. This $337 \mathrm{~cm}^{-1}$ progression is congested with lower intensity peaks, sometimes hardly discernible from the noise, spaced fairly regularly about the progression members. For example, peaks $1^{\prime}, 2^{\prime}, \ldots, 5^{\prime}$ are found $-80 \mathrm{~cm}^{-1}$ to the red of peaks $0, \ldots 4$, respectively. Peaks $a_{1}, a_{2}$ and $a_{3}$ are found $-80 \mathrm{~cm}^{-1}$ to the blue and peaks $b_{1}, b_{2}$, and $b_{3}$ are found roughly $160 \mathrm{~cm}^{-1}$ to the blue of peaks 1,2 , and 3 , respectively. There are no corresponding peaks $a_{0}$ or $b_{0}$ to the blue of peak 0 . Additional structure occurs to the red of the origin. The fairly intense peak $1^{\prime \prime}$ is found $128 \mathrm{~cm}^{-1}$ to the red of peak 0 , and two very low intensity peaks, labelled $a$ and $b$, occur at -280 and $565 \mathrm{~cm}^{-1}$ to the red of peak 0 , respectively. The peak labels, positions and relative energies are summarized in Table (6.1).

\section{Analysis and Discussion}

\section{A. Assignment of electronic and vibrational structure}

The highest level calculations (QCISD(T)/6-31G') performed by Rohlting and Raghavachari ${ }^{17}$ have predicted both the anion and neutral ground states to be isosceles $\left(C_{2 v}\right)$ triangles where the $\mathrm{Si}-\mathrm{Si}-\mathrm{Si}$ bond angle $(\theta)$ is $65.2^{\circ}$ for the ${ }^{2} \mathrm{~A}_{1}$ anion ground state and $79.6^{\circ}$ for the ${ }^{1} A_{1}$ neutral ground state. The neutrai structure is the result of Jahn-Teller distortion of the ' $E$ ' $\left(D_{3 h}\right)$ state, which is formed by the

$$
\left(1 a_{1}\right)^{2}\left(1 e^{\prime}\right)^{4}\left(2 a_{1}{ }^{2}\right)^{2}\left(1 a_{2}{ }^{\prime}\right)^{2}\left(2 e^{\prime}\right)^{2}
$$

configuration. This distortion to $\mathrm{C}_{2 v}$ geometry, which causes the degenerate $\mathrm{e}^{\prime}$ orbitals to split into $a_{1}$ and $b_{2}$ orbitals, results in the ${ }^{1} A_{1}$ state with the 


$$
\left(a_{1}\right)^{2}\left(b_{2}\right)^{2}\left(a_{1}\right)^{2}\left(a_{1}\right)^{2}\left(b_{1}\right)^{2}\left(b_{2}\right)^{2}
$$

configuration and a more acute ${ }^{1} \mathrm{~B}_{2}$ state. The ${ }^{1} \mathrm{~A}_{1}$ state is calculated to be much lower in energy than the ${ }^{1} B_{2}$ state due to second order Jahn-Teller stabilization effects. The undistorted $\left(D_{3 h}\right){ }^{3} A_{2}$ state, which has been calculated to be nearly degenerate with the ${ }^{1} A_{1}$ state, $9,10,17$ has the orbital occupancy of config. (6.1) with parallel spins in the '2e' orbitals.

The anion ground state is obtained by adding an electron to the $2 e^{\prime}$ orbital of config. (6.1), resulting in a ${ }^{2} E^{\prime}$ state. This state is also subject to Jahn-Teller distortion to the previously mentioned ${ }^{2} A_{1}$ state with the orbital occupation

$$
\ldots\left(a_{1}\right)^{2}\left(b_{1}\right)^{2}\left(b_{2}\right)^{2}\left(a_{1}\right)^{1}
$$

and a ${ }^{2} \mathrm{~B}_{2}$ state with $\theta=57^{\circ}$. At the Hartree-Fock level, the ${ }^{2} \mathrm{~B}_{2}$ is the ground state and the ${ }^{2} \mathrm{~A}_{1}$ is the saddle point on the typical J-T distorred potential, but upon including electron correlation effects, these are reversed and the ${ }^{2} A_{1}$ becomes more stable at the QCISD(T) level by $\sim 160$ $\mathrm{cm}^{-1} .{ }^{16,17}$ This energy difference corresponds to the pseudorotation barrier.

The $337 \mathrm{~cm}^{-1}$ progression seen both in the PES [Figure (6.1)] and, more detinitively, in the ZEKE spectrum appears to be due to transitions between the ground anion state and vibrational levels in the ground neutral state. Because of the large bond angle difference between the ${ }^{2} A_{1}$ anion and the ${ }^{1} A_{1}$ neutral, the symmetric bend mode $\left(v_{2}\right)$ should be the most active in the spectrum. However, this mode is consistently calculated to be approximately 150 to $170 \mathrm{~cm}^{-1}, 9,10,14,17$ which is approximately half the observed frequency. The symmetric stretch mode $\left(v_{1}\right)$, which could also be active in the spectrum, has a calculated trequency of 551 $\mathrm{cm}^{-1}$, much higher than the observed frequency. This suggests that the band in our spectrum does not represent transitions to the ${ }^{1} A_{1}$ neutral state. 
Alternatively, the calculated $e^{\prime}$ frequency of the ${ }^{3} A_{2}{ }^{\prime}\left(D_{3 h}\right)$ state is in better agreement with the spacing in the observed progression. Raghavachari ${ }^{17}$ and Schaefer ${ }^{9}$ calculated a trequency of approximately $285 \mathrm{~cm}^{-1}$ tor the $e^{\prime}$ mode using the QCISD(T) and SCF methods. respectively. Density functional studies on the $D_{3 h}$ structure performed by Dixon, ${ }^{12}$ Dupuis, ${ }^{13}$ and DePristo ${ }^{14}$ yielded values around $340 \mathrm{~cm}^{-1}$ for the $\mathrm{e}^{\prime}$ frequency. Because the calculated $\mathrm{e}^{\prime}$ frequency is in better agreement than either of the ${ }^{1} A_{1}$ frequencies with the observed $337 \mathrm{~cm}^{-1}$ progression, we believe that the band in the ZEKE spectrum is due to the ${ }^{3} A_{2}^{\prime}+e^{-} \leftarrow{ }^{2} A_{\text {; }}$, transition and that the $337 \mathrm{~cm}^{-1}$ progression can be assigned to transitions to levels in the $v_{2}\left(e^{\prime}\right)$ mode of the neutral; this was first suggested in Dixon's comparison of ab initio frequencies with the experimental photoelectron and emission spectra. ${ }^{12}$ Although long progressions seen in photodetachment spectra are generally only in totally symmetric modes of the neutral, one component of the $\mathrm{e}^{\prime}$ vibration transforms as the symmetric $\mathrm{v}_{2}\left(\mathrm{a}_{1}\right)$ bend mode of the $\mathrm{c}_{2 v}$ anion. Therefore, in the rigid molecule limit (the first situation that we will treat), transitions from the totally symmetric ground state of the anion to any quanta in the neutral mode are allowed. Hence we assign each peak $n$ to a $2_{0}^{n}$ transition.

Based on further comparison of the ZEKE spectrum with $a b$ initio frequency calculafions, we can assign peak $b_{1}$ to the $1_{0}^{1}$ transition, yielding $501 \pm 10 \mathrm{~cm}^{-1}$ for the neutral $v_{1}$ frequency. This is in good agreement with all of the calculations for the symmetric stretch $\left(v_{1}\right)$ frequency of the ${ }^{3} A_{2}$ state, but the density functional studies again give the closest value, approximately $500 \mathrm{~cm}^{-1}$. Peaks $b_{2}, \ldots, b_{4}$ can then be assigned $10 v_{1}+n v_{2} \cdot 1_{0}^{1} 2_{0}^{n}$ ) combination bands.

The structure found ti lower photon energy from the origin was found to depend on ion source conditions, suggesting that they are transitions from vibrationally excited levels of the anion (hot banf transitions). The most intense hot bands are those labeled $0^{\prime}$ and $1^{\prime \prime}$, with peaks $b$ and $a$ being less intense. Because the $v_{2}$ mode is the most active in the spectrum, we can tentatively assign $0^{\prime}$ to the $2_{i}^{\circ}$ transition. The position of peak $0^{\prime}$ with respect to the origin 
would give an anion fregtency of $385 \mathrm{~cm}^{-1}$, which is in poor agreement with the calculated value, $297 \mathrm{~cm}^{-1} .{ }^{17}$ Peak $b$, found $565 \mathrm{~cm}^{-1}$ from the origin, can be tentatively assigned to the $1_{1}^{0}$ transition, giving the anion $v_{1}$ frequency in fairly good agreement with the $533 \mathrm{~cm}^{-1}$ calcuiated frequency. ${ }^{17}$

The remaining structure found to higher photon energy from the origin, such as peaks $n^{\prime}$ and $a_{n}$, also appears to be due to transitions from excited vibrational levels in the anion. Based on the $v_{1}$ and $v_{2}$ anion and neutral frequencies determined above, seouence bands originating from either the $v_{2}=1$ or $v_{3}=1$ anion levels should fall to the red of the corresponding $2_{0}^{n}$ transition, in the energetic vicinity of peaks $n^{\prime}$. Peaks $a_{n}$, slightly to the blue of each $2_{0}^{n}$ transition. could then be sequence bands in the $v_{3}$ mode, giving an anion frequency of less than $337 \mathrm{~cm}^{-1}$ for this mode.

Since we have determined that neutral $\mathrm{Si}_{3}$ state in the ZEKE spectrum has $\mathrm{D}_{3 h}$ symmetry, we can attempt to extract information regarding the anion geometry most simply by assuming that the anion has a rigid, $\mathrm{C}_{2 v}$ structure. With this assumption, we can treat the profile of the progression by performing spectral simulations within the Franck-Condon approximation, where we assume the vibrational wave functions of the anion and neutral are the products of three harmonic oscillators representing the three normal modes. The intensity of a transition between vibrational levels in the anion and neutral is theretore proportional to the FranckCondon iactors of the two levels. The neutral and anion frequencies are fixed by the above assignments; note that the neutral is treated as a $C_{2 v}$ species with $v_{2}=v_{3}=337 \mathrm{~cm}^{-1}$ (where $v_{3}$ in this instance corresponds to the component of the neutral degenerate mode that transforms as $\mathrm{b}_{2}$ in $\mathrm{C}_{2 v}$ geometry).

This "rigid anion" simulation is shown in the second panel of Figure (6.3). The profile of the $337 \mathrm{~cm}^{-1}$ progression was well matched by assuming a normal mode displacement (from anion to neutral) of $\Delta Q_{2}=0.095 \AA$ amu $u^{1 / 2}$, given that the anion frequency for this mode is 385 $\mathrm{cm}^{-1}$. The lower intensity $b_{n}$ peaks (the $1_{0}^{1} 2_{0}^{n}$ transitions) were accounted for by assuming $\Delta Q_{1}=$ 
$0.03 \mathrm{~A} \cdot \mathrm{amu} \mathbf{k}^{12}$. These two displacements compare well to the predicted displacements between the anion and neutral based on Raghavachari's geometry calculations and our own determination of the normal mode coordinates: $\Delta Q_{2}=0.117 \AA$ amu $u^{1 / 2}$ and $\Delta Q_{1}=0.03 \AA$ amu $1 / 2$.

The intensities of all transitions originating from vibrationally excited levels in the anion are dependent on vibrational temperature. In general, the lower frequency modes tend to have lower temperatures than the higher frequency modes. This is not unusual since different modes are expected to cool with different efficiencies in a molecular beam. Temperatures for the various levels are included in the figure caption.

While the geometry changes between the anion and neutral determined from this simpie treatment are ir good agreement with the calculated geometries, the rigid anion simulation exhibits several inadequacies in reproducing the positions and intensities of the sequence bands, namely, the $a_{n}$ and $n^{\prime}$ peaks. First, the difference in frequencies between the anizn and neutral symmetric bend produces sequence bands of the type $2_{\uparrow}^{n+1}$, marked with circles on the simulation shown in Figure (6.3). These occur approximately $50 \mathrm{~cm}^{-1}$ to the red of the main $2_{0}^{\text {ng }}$ peaks. Also contributing to the intensity of these simulated peaks are $1_{1}^{1} 2_{0}^{n}$ sequence bands. which occur $65 \mathrm{~cm}^{-1}$ to the red of the $2_{0}^{n}$ peaks. This is in contrast with the actual position of peaks $n^{\prime}$, which are found $80 \mathrm{~cm}^{-1}$ to the red of the main peaks. This discrepancy is small but significant. Additionally, as mentioned above, peaks $a_{1}, a_{2}$, and $a_{3}$ can be proposed as $2_{0}^{n} 3_{1}$; transitions, which would yield an anion $v_{3}$ frequency of approximately $270 \mathrm{~cm}^{-1}$ (the frequency used in the rigid anion simulation shown). However, this also produces a fairly intense sequence band to the blue of the origin, marked with a star, which is hardly present in the actual spectrum. There is aiso no structure in the experimental spectrum where the $3_{2}^{2}$ sequence bands are predicted to lie (these are marked with an " $x$ " on the Franck-Condon sir "ilation). Finally, this analysis implies anion $v_{2}$ and $v_{3}$ frequencies of 385 and $270 \mathrm{~cm}^{-1}$, respectively. This frequency ordering is unusual for a $C_{2 v}$ molecule with a bond angle greater than $60^{\circ}$, and is the reverse of the calculated ordering $\left(v_{2}=297 \mathrm{~cm}^{-1}, v_{3}=370 \mathrm{~cm}^{-1}\right),{ }^{17}$ Taken together, all of 
these problems suggest that the analysis is partially flawed, and that it is naive to treat the arion as a simple, rigid $\mathrm{C}_{2 v}$ molecule with uncoupled modes.

\section{B. Inclusion of Jahn-Teller distortion in $\mathrm{Si}_{3}{ }^{-}$}

Since the barrier to pseudorotation in $\mathrm{Si}_{3}{ }^{-}$is calculated to be only $160 \mathrm{~cm}^{-1}$, an alternative approach to that outlined above is to analyze the ZEKE spectrum by treating the anion as fluxional, rather than as a rigid molecule. This situation arises in symmetric molecules having a set of spatially degenerate electronic states which are vibronically coupled by a degenerate vibration. The end result is a Jahn-Teller distortion of the molecule along the corresponding normal coordinate, removing the electronic degeneracy with the lowering of symmetry. In the case of $\mathrm{Si}_{3}$, we have a species distorted from a symmetric $\mathrm{D}_{3 n}$ equilibrium geometry because the $\mathrm{e}^{\prime}$ degenerate vibration couples the two components of the ${ }^{2} \mathrm{E}$ ' electronic state (see section A).

The $\mathrm{E} \times \mathrm{e}$ coupling problem can be approached most simply by assuming that the

vibronic coupling term is linear with respect to the $e^{\prime}$ nomal coordinate, i.e., no barrier to pseudorotation. In the basis of the two electronic states, and with the degenerate twodimensional harmonic oscillator expressed in polar coordinates $(\rho, \phi)$, the vibrational energies and wave functions are determined from,

$$
\left[\begin{array}{cc}
H_{0}-E & k \rho e^{-i \varphi} \\
k \rho e^{i 0} & H_{0}-E
\end{array}\right]\left[\begin{array}{l}
\chi_{+} \\
\chi_{-}
\end{array}\right]=0,
$$

where $H_{0}$ is the unperturbed Hamiltonian, $k$ is a unitless parameter such that $\omega_{2} k^{2} / 2$ is the stabilization energy from the distortion, $\omega_{2}$, in this instance, is the harmonic frequency of the $e^{\prime}$ mode in the undistorted molecule (often referred to as $\omega_{0}$ ), and $\chi_{ \pm}$are the undistorted 
vibrational wave functions associated with the two electronic states. The implications of ihis have been studied extensively by Longuet-Higgens, ${ }^{21}$ and Whetten, 22 for example, and this treatment has been anplied to, among other molecules, the analysis of $\mathrm{Cu}_{3},{ }^{23} \mathrm{Na}_{3},{ }^{24} \mathrm{Ag}_{3},{ }^{25}$ and $\mathrm{L}_{3}{ }^{26}$ spectra. These studies demonstrate that even for moderate values of the distortion parameter $k$, one expects an extended progression in the $e^{\prime}$ mode for a transition between a Jahn-Teller distorted state and an undistorted $\mathrm{D}_{3 h}$ state. This is exactly the situation for the $\mathrm{Si}_{3}$ $\left({ }^{3} \mathrm{~A}_{2}\right) \leftarrow \mathrm{Si}_{3} \cdot$ transition in our ZEKE spectrum. The key question, then, is whether the smaller peak energies and intensities can be better explained assuming a Jahn-Teller distorted anion rather than a rigid, $\mathrm{C}_{2 \mathrm{v}}$ anion.

We first consider the anion energy levels. For $k$ sufficiently large, the vibrational energy level pattems should approximately follow the familiar formula for a freely pseudorotating species, ${ }^{21}$

$$
E=\hbar \omega_{1}\left(v_{1}+\frac{1}{2}\right)+\hbar \omega_{2}\left(v_{2}+\frac{1}{2}\right)+A j^{2},
$$

where $\omega_{1}$ is the symmetric stretch frequency, $\omega_{2}$ is as defined above, $v_{1}$ and $v_{2}$ are the quantum numbers associated with $\omega_{1}$ and $\omega_{2}$, and $j$ is the half-integer pseudorotation quantum number. The pseudorotation constant $A$ is inversely proportional to the reduced mass and the square of the equilibrium distortion along the $e^{\prime}$ coordinate $\left(\hbar^{2} / 2 \mu p_{o}^{2}\right)$. This produces an energy level pattern similar to that shown in Figure (6.4), where the anion levels are labelled $\left(v_{1}, v_{2}, j\right)$. Deviations from the energy level pattern of Eq. (6.2) occur when $k^{2}$ is small (very small distortion), or when there is any barrier to pseudorotation.

In the limit of linear vibronic coupling [Eq. (6.1)], transitions from a particular $j$ level of the anion are allowed to $l=j \pm 1 / 2$ levels in the neutral, where $l$ is the vibrational angular momentum for the $\left(D_{3 h}\right)$ neutral species. The allowed neutral $\leftarrow$ anion transitions (and the forbidden transition, peak a, which is discussed below) are indicated by arrows in Figure (6.4). This figure 
suggests an alternate explanation for several of the lower intensity peaks in the ZEKE spectrum, namely, that they are "hot bands" originating from excited pseudo-rotational states of the anion, rather than from excited vibrational levels as assumed in the "rigid anion" Franck-Condon analysis. Thus, the sequence transitions between the $0,0,3 / 2$ level and the neutral $v_{2} \geq 1$ levels would account for peaks $2^{\prime}-4^{\prime}$, etc., while the transitions from the $0,0,5 / 2$ level to the neutral $v_{2}$ $\geq 2$ levels account for peaks $a_{1}-a_{3}$.

We can determine if this assignment is reasonable by computing the pseudorotation constant $A$ in Eqn. (6.2) from the energy difference between peaks 1 and $2^{\prime}$ in the experimental spectrum, assuming that these are the $0,1,1 \leftarrow 0,0,1 / 2$ and the $0,1,1 \leftarrow 0,0,3 / 2$ transitions, respectively. This difference is $80 \mathrm{~cm}^{-1}$, yielding $A=40 \mathrm{~cm}^{-1}$ and $\sqrt{\mu} \rho_{0}=0.11 \AA \cdot \mathrm{amu}^{1 / 2}$. The latter value is very similar to the $0.095 \dot{A}$-amu ${ }^{1 / 2}$ nornal coordinate displacement determined from the Franck-Condon simulation as well as the ab initio displacement, indicating that our value for $A$ is certainly acceptable. However, with such a small distortion, Eqn. (6.2) may not give a good approximation of the actual energy levels.

To proceed further, we need to simulate the ZEKE spectrum including the effects of Jahn-Teller coupling in the anion. This requires that we solve Eqn. (6.1) for the anion wave functions and energy levels, varying $k$ to best match the intensity pattem of the main bending progression. The energy levels are abtained in terms of $\omega_{2}$, the (undistorted) harmonic $e^{\prime}$ frequency, which can then be extracted by scaling the simulated spectrum to match the spacing between peaks 0 and $0^{\prime}$. Intensities are determined by calculating the overlap between the anion and neutral nuclear wave functions; as before, the symmetric stretch mode is assumed to be uncoupled to the $e^{\prime}$ mode. The anion bend eigenfunctions of Eqn. (6.1) are linear combinations of hamonic oscillator wave functions with frequency $\omega_{2}$, and, for the purpose of intensity calculations only, the neutral $e^{\prime}$ wave functions are taken to be harmonic with frequency $\omega_{2}$. As long as the neutral $e^{\prime}$ frequency of $337 \mathrm{~cm}^{-1}$ differs only slightly from $\omega_{2}$ (which it does; see below), this approximation will have a negligible effect on the calculated intensities. 
Transitions from levels as high as the $(1,0,3 / 2)$ level $\left(-650 \mathrm{~cm}^{-1}\right)$ were considered in these simulations.

The best simulation, shown in the third panel of Figure (6.3), was obtained with $k=1.9$. As expected, such a small $k$ gives vibrational and pseudorotational energy levels deviating slightly trom Eqn. (6.2). For instance, the separation of the first two $j=1 / 2$ levels is $-1.1 \mathrm{\omega}_{2}$ las opposed to $\omega_{2}$, per Eqn. (6.2)] which should equal the $385 \mathrm{~cm}^{-1}$ frequency determined from hot band $\sigma^{\prime}$. From this we find $\omega_{2}=350 \mathrm{~cm}^{-1}$, which, as would be expected, falls between the calculated $a_{1}\left(297 \mathrm{~cm}^{-1}\right)$ and $b_{2}\left(370 \mathrm{~cm}^{-1}\right)$ frequencies for the distorted ${ }^{2} A_{1}$ state. ${ }^{17}$ Moreover, the energies of the $j=5 / 2$ and $j=3 / 2$ levels with respect to the ground $j=1 / 2$ level are not at a ratio of $3: 1$, as given by Eqn. $(6.2)$.

The overalt agreement with the experimental spectrum is reasonable, supporting the assignments discussed above. One nice feature is that the peak marked with the $z$ in the rigid anion simulation is absent, in better agreement with the experiment; this peak would correspond to the $(0,1,1) \leftarrow(0,05 / 2)$ transition, which is forbidden by the $l=j \pm 1 / 2$ selection rule. However, the linear Jahn-Teller simulation still has some shortcomings. The energy separation between the two lowest levels of the anion, the $(0,0,1 / 2)$ and $(0,0,3 / 2)$ levels, is $0.311 \omega_{2}\left(110 \mathrm{~cm}^{-1}\right)$. Consequently, transitions originating from the $(0,0,3 / 2)$ level, marked with circles in the simulation, are slightly to the red of the experimental sequence bands, peaks $2^{\prime}-4{ }^{\prime}$. This small overestimation of the $j=3 / 2$ level has also been seen in other calculations for several of the excited states of $\mathrm{Na}_{3} \cdot{ }^{27}$ As with the "rigid anion" simulation, $\Delta v_{1}=0$ sequence bands from the $1,0,1 / 2$ anion level fall approximately 50 to $60 \mathrm{~cm}^{-1}$ to the red of the main progression members, so there are two peaks to the red of each of the main peaks $1 \cdot 3$, as opposed to one in the experimental spectrum. Finally, the small peak $a$, found : $0 \mathrm{~cm}^{-1}$ to the red of the origin, energetically appears to be the transition from the $(0,0,5 / 2)$ level to the ground vibrational level of the neutral, but this transition is not allowed becalise of the $(l, j)$ selection rule. 
We can, however, attempt to better align the $n^{\prime}$ sequence bands both by increasing $k$ slightly, which lowers the energy of the $(0,0,3 / 2)$ level. and by including the quadratic coupling term $\frac{1}{2} g \rho^{2} e^{ \pm 2 i \theta}$ in Eqn. (6.1). This results in a barrier to pseudorotation given by $\mathrm{E}_{\mathrm{bcc}}=\mathrm{gk}^{2} \omega_{i} /\left(1-\mathrm{g}^{2}\right), 21$ which splits the degenerate $j=3 / 2$ levels substantially.22 Additionally, this quadratic term couples the $j=1 / 2$ level with the $j=5 / 2$ level (through the $\rho^{2}$ term), resulting in nonzero intensity for the $(0,0,0) \leftarrow(0,0,5 / 2)$ transition believed responsible for peak $a$. The effects of these two modifications are shown in the bottom panel of Figure (6.3). For this simulation, $\mathrm{k}=2.0$, and $\mathrm{g}=0.005$ in the quadratic coupling term. With these parameters, $E_{10 c}=$ $7 \mathrm{~cm}^{-1}$, the $(0,0,3 / 2)$ level is split by $20 \mathrm{~cm}^{-1}$ [the resulting levels are labelled $\left(0,0,3 / 2^{ \pm}\right)$in Table (6.1)], and the stabilization energy is $725 \mathrm{~cm}^{-1}$. We now observe only one resolved peak to the red of each of the peaks $1-3$, and the placement agrees with the experimental peaks $2^{\prime}-4^{\prime}$. The simulation shows several weak features which appear to correspond to peak a as well as some of the other peaks to the red of the origin.

We also attempted to adjust the linear and quadratic parameters to give a greater barrier to pseudorotavion, as it has been calculated to be $160 \mathrm{~cm}^{-i}$. However, the spectrum could not be simulated assuming such a high barrier. For instance, with $k=1.8$ and $\mathrm{g}$ determined using $E_{\text {loc }}=160 \mathrm{~cm}^{-1}$, the simulated bend progression was tar too extended. We also varied $\mathrm{k}$ and $\mathrm{g}$ to give a larger barrier and a shorter progression, but the sequence band structure obtained from these variations was in much worse agreement with the experimental sequence band structure.

While none of the three simulations shown in Figure (6.3) exactly match the experimental spectrum (most notably, peak $1 "$ is still unassigned), the quadratic Jahn-Teller treatment is superior to the other two. The "rigid anion" treatment did not correctly match the experimental sequence band transitions, and the anion $v_{2}$ and $v_{3}$ frequencies determined from this analysis were the reverse of the calculated values. In the linear Jahn-Teller iimit, several of the simulated sequence peaks were again mispositioned, but the overall profile of the spectrum 
was better matched. The quadratic Jahn-Teller coupling treatment yielde-t a closer overall match of the simulated and experimental peaks. Moreover, the quadratic model is consistent with the ab initio prediction of a small barrier to pseudorotation, although we find the barrier to be even lower than calculated. ihe peak assignments based on the quadratic Jahn-Teller simulations are given in Table (6.1). Several of the peaks consist of overlapping transitions, and in these cases, the transitions are listed in order of descending contribution. Note that the composition of peaks $2^{\prime}-4^{\prime}$ is quite different from peaks $0^{\prime}$ and $1^{\prime}$, even though they appear to be part of a single progression. Also note that there are nn irianges in the assignments of peaks $n, b_{n}$, and $0^{\prime}$ from the rigid anion analysis of section $A$, although the notation is somewhat different.

\section{Role of the ${ }^{1} \mathbf{A}_{1}$ state}

Figure (6.1) shows that the extent of the ZEKE spectrum is considerably less than that of band $X$ in the PES. Moreover, the ZEKE intensity falls off dramatically just where band $X$ degenerates into a mass of unresolved signal at lower electron kinetic energies. his congested signal in the PES is most likely due to transitions to the ${ }^{1} A_{1}$ state of the nevitial. From the calculated anion and neutral geometries and the form of the no.mal coordinates, we detemine the normal mode displacement to be $0.043 \dot{A} \cdot a m u^{1 / 2}$ and $0.314 A \cdot a m u^{1 / 2}$ for the $v_{1}$ and $v_{2}$ modes, respectively. Displacement of this magnitude would produce a much longer progression in the $v_{2}$ mode (in addition to significant activation in the $v_{1}$ mode) than what is observed in the ZEKE spectrum of the $X$ band.

As a first order approach to determine the extent to which the ${ }^{1} A_{1}+F{ }^{-} \leftarrow{ }^{2} A_{1}$ transition contributes to the PES $X$ band, we can simulate this transition at an arbitrary origin and then convolute it with the PES experimental resolution. We can then add it to the simulation of the $-m_{2}+e^{-} \leftarrow{ }^{2} A_{1}$ transition, also convoluted with the PES resolution, adjusting the origin and the 
electronic transition moment of the ${ }^{1} A_{1}$ simulation until the profile of the PES $X$ band is matched. Figure (6.5) shows the result of the added simulations with the origin of the ${ }^{1} A_{1}+e^{-} \leftarrow{ }^{2} A_{1}$ transition placed $10 \mathrm{meV}$ below the origin of the ${ }^{3} \mathrm{~A}_{2}{ }^{\prime}+\mathrm{e}^{-} \leftarrow{ }^{2} \mathrm{~A}_{1}$ transition. However, we hesitate to make any final comment as to the actual ground state based on this somewhat crude analysis.

It still remains that thie transition to the ${ }^{1} A_{1}$ state is not apparent in the ZEKE spectrum. This state is formed by removing the lone $a_{1}$ electron from the ion [reler to config. (6.3)], while the ${ }^{~} \mathrm{~A}_{2}$ ' state is formed by removing a $b_{2}$ electron. Following the Wigner threstudd law, 28 and the selection rules developed by Reed et al., ${ }^{29}$ the total photodetachment cross sections near threshold for both of these states should be proportional to $\sigma_{v}\left(E_{h v}-E_{\text {treeshold }}\right)^{1 / 2}$, which rises sharply just above photodetachment threshold (s-wave detachment). However, it appears to be the case that $\sigma_{0}$ near threshold for the former transition is considerably smaller than for the latter. Interestingly, band $\mathrm{B}$ in the $\mathrm{Si}_{3}{ }^{-}$photoelectron spectrum, which was assigned to the ${ }^{3} A_{1}+e^{-} \leftarrow{ }^{2} A_{1}$ transition, ${ }^{17}$ also involves the removal of a lower-lying $a_{1}$ electron. While band $B$ is the most prominent feature in the PES, the cross section near threshold for this band was too small to obtain higher resolution ZEKE spectra.

\section{v. Conclusions}

The analysis of the extensive vibrational structure in the ZEKE spectrum of $\mathrm{Si}_{3}$. shows that the spectrum is due to the ${ }^{3} \mathrm{~A}_{2}{ }^{\prime}+\mathrm{e}^{-} \leftarrow{ }^{2} \mathrm{~A}_{1}$ transition. Progressions in the bending and symmetric stretch modes of the neutral are observed, yielding frequencies of $.37 \mathrm{~cm}^{-1}$ and $530 \mathrm{~cm}^{-1}$, respectively. A comparisor of these frequencies with ab initio values shows that agreement is best with several of the density functional calculations. The ZEKE spectrum shows several smaller features in addition to the main progressions. These are best explained 
by treating the anion as a fluxional species with some pseudorotational excitation; the smaller peaks are then assigned to sequence bands originating from these excited levels of the anion.

A comparison of the $\mathrm{Si}_{3}{ }^{-}$PES and ZEKE spectrum suggests that Band $X$ in the PES consists of overlapping transitions to the neutral ${ }^{1} \mathrm{~A}_{1}$ and ${ }^{3} \mathrm{~A}_{2}$ ' states, but the transitions to the ${ }^{1} A_{1}$ state do not appear in the ZEKE spectrum. This is presumably due to a small photodetachment cross section for the transition to the singlet state near the detachment threshold. Unfortunately, this means we cannot detemine which state of $\mathrm{Si}_{3}$ is the true ground state, although simulations of band $X$ using $a b$ initio geometries suggest that it is the singlet stais.

\section{Acknowledgments}

This work was supported under NSF Grant No. DMR-9201159. We would like to thank Michel Dupuis and Celeste McMichael Rohliing for helpful conversations. 
1P. W. Merril, Publ. Astron. Sac. Pac. 38, 175 (1926); R. F. Sanford, Astrophys. J. 111, 262 (1950); B. Kleman, Astrophys. J. 123, 162 (1950) and references therein.

2P. Ho and W. G. Breiland, App. Phys. Lett. 44, 51 (1984).

${ }^{3}$ R. O. Jones, Phys. Rev. A 32, 2589 (1985); K. Raghavarhari and V. Logovinsky, Phys. Rev. Lett. 55, 26 (1985); G. Pacchioni and J. Koutecky, J. Chem. Phys. 84, 3301 (1986); Z. Slanina, Chem. Phys. Lett. 131, 420 (1986); D. Tomanek and M. A. Schluter, Phys. Rev. Lett. 56, 1055 (1986); K. Balasubramanian, Chem. Phys. Lett. 135, 283, (1987); K. Raghavachari, Z. Phys. D 1261 (1989); C. M. Rohlfing and K Raghavachari, Chem. Phys. Lett. 167, 559 (1990); S. D. Li, R. L. Johnston, and J. N. Murrell, J. Chem. Soc.- Faraday Trans. 88, 1229 (1992).

${ }^{4}$ A. E. Douglas, Can. J. Phys. 33, 801 (1955); R. D. Verma and P. A. Warsop, Can. J. Phys. 41, 152 (1963); I. Dubois and H. Leclerq, Can. J. Phys. 49, 3053 (1971); S. P. Davis and J. W. Brautt, J. Opt. Soc. Am. B 4, 20 (1987); M. R. Nimlos, L. B. Harding, and G. B. Ellison, J. Chem. Phys. 87, 5116 (1987).

5T. N. Kitsopoulos, C. J. Chick, Y. Zhao, and D. M. Neumark, J. Chem. Phys. 95,1441 (1991); C. C. Arnold, T. N. Kitsopoulos, and D. M. Neumark, J. Chem. Phys. 99, 766 (1993).

6T. N. Kitsopoulos, C. J. Chick, A. Weaver, and D. M. Neumark, J. Chem. Phys. 93, 6108 (1990).

${ }^{7}$ C. B. Winstead, K. X. He, D. Grantier, T. Hammond, and J. L. Gole, Chem. Phys. Lett. 181, 222 (1991).

${ }^{8}$ C. C. Amold and D. M. Neumark, J. Chem. Phys. 99, in press.

${ }^{9}$ R. S. Grev and H. F. Schaefer, Chem. Phys. Lett. 119, 111 (1985).

10K. Raghavachari, J. Chem. Phys. 83, 3520 (1985).

${ }^{11}$ L. Gausset, G. Herzberg, A. Lagerquist, and B. Rosen, Discuss. Faraday Soc. 35, 113 (1963); Astrophys. J. 142, 45 (1967). 
12 D. A. Dixon and J. L. Gole, Chem. Phys. l.ett. 188, 560 (1992).

13M. Dupuis (private communication).

14R. Fournier, S. B. Sinnott, and A. DePristo, J. Chem. Phys. 97, 4149 (1992).

15 J. R. Sabin, J. Oddershede, G. H. F. Diercksen, and N. E. Gruner, J. Chem. Phys. 84, 354 (1986); ; K. Raghavachari, J. Chem. Phys. 84, 5672 (1986); K. Balasubramanian, Chem. Phys. Le't. 125, 400 (1986).

16K. Raghavachari and C. M. Rohlfing, J. Chem. Phys. 94, 3670 (1991).

${ }^{17} \mathrm{C}$. M. Rohlfing and K. Raghavachari, J. Chem. Phys. 96, 2114 (1992).

${ }^{18}$ O. Cheshnovshy, S. H. Yang, C. L. Pettiette, M. J. Craycratt, Y. Liu, and R. E. Smalley, Chem. Phys. Lett. 138, 119 (1987).

19T. N. Kitsopoulos, I. M. Waller, J. G. Loeser, and D. M. Neumark, Chem. Phys. Lett. 159, 300 (1989); C. C. Amold, Y. Zhao, T. N. Kitsopoulos, and D. M. Neumark, J. Chem. Phys. 97. 6121 (1992).

20K. Müller-Dethlets, M. Sander, and E. W. Schlag, Z Naturforsch 39a, 1089 (1984); Chem. Phys. Lett. 12, 291 (1984); K. Müller-Dethlefs and E. W. Schlag, Ann. Rev. Phys. Chem. 42, 109 (1991).

${ }^{21}$ H. C. Longuet-Higgens, U. Opik, M. H. L. Price, and R. A. Sack, Proc. R. Soc. London Ser. A 244, 1 (1958); H. C. Longuet-Higgens, Adv. Spectrosc. 2, 429 (1961).

22R. L. Whetten, G. S. Ezra, and E. R. Grant, Ann. Rev. Phys. Chem. 36, 277 (1985); R. L. Whetter, K. S. Haber, abd E. R. Grant, J. Chem. Phys. 84, 1271 (1985).

23M. D. Morse, Chem. Phys, Lett. 133, 8 (1987); E. A. Rohjfing and J. J. Valentini, Chem. Phys. Lett. 126, 113 (1986); M. D. Morse, J. B. Hopkins, P. R. R. Langridge-Smith and R. E. Smalley, J. Chem. Phys. 79, 5316 (1983); M. Moskovits, Chem. Phys. Lett. 118, 111 (1985).

${ }^{24}$ G. Delacrétaz, E. R. Grand, R. L. Whetten, L. Wöste, and J. Zwanziger, Phys. Rev. Lett. 56, 2598 (1986). 
25K. Laitting, P. Y. Cheng and M. A. Duncan, Chem. Phys. Lett. 152, 341 (1988); P. Y. Cheng and M. A. Duncan, Chem. Phys. Lett. 156, 420 (1989); A. M. Ellis, E. S. J. Robles and T. A. Miller, Chem. Phys. Lett. 201, 132 (1993).

26Ph. Dugourd, J. Chevaleyre, M. Broyer, J. P. Wolf, and L. Wōste, Chem. Phys. Lett. 175, 555 (1990).

${ }^{27}$ G. Delecrétaz, E. Grant, R. L. Whetten, L. Wöste, and J. Zwanziger, Phys. Rev. Lett. 56, 2598 (1986); M. Broyer, G. Delecrétaz, G. -L. Ni, R. L. Whetten, J. P. Wolf, and L. Wöste, J. Chem. Phys. 90, 843 (1989).

28 E. P. Wigner, Phys Rev. 73, 1003 (1948).

${ }^{29}$ K. J. Reed, A. H. Zimmerman, H. C. Andersen, and J. I. Brauman, J. Chem. Phys. 64, 1368 (1976). 
Table (6.1) Peak positions, relative energies, and proposed assignments for $\mathrm{Si}_{3} \mathrm{ZEKE}^{-}$ spectrum. The assignments, shown as ${ }^{3} A_{2}^{\prime}\left(v_{1}, v_{e^{\prime}}, m\right) \leftarrow{ }^{2} A_{1}\left(v_{1}, v_{2}, j\right)$, are based on quadratic Jahn-Teller spectral simulation (see text). Uncertainties in peak positions are given in parentheses. When more than one transition contributes to a peak, the transitions are listed in order of decreasing contribution.

\begin{tabular}{|c|c|c|c|}
\hline Peak & $\begin{array}{l}\text { Position } \\
(\mathrm{nm})\end{array}$ & $\begin{array}{l}\text { Relative Energy } \\
\left(\mathrm{cm}^{-1}\right)\end{array}$ & $\begin{array}{l}\text { Possible transition in order of descending } \\
\text { intensity }\end{array}$ \\
\hline b & 555.55 & $-565(15)$ & $(0,0,0) \leftarrow(1,0,1 / 2)$ \\
\hline $0^{\prime}$ & 550.05 & $-385(12)$ & $(0,0,0) \leftarrow(0,1,1 / 2)$ \\
\hline$a$ & 546.95 & $-282(10)$ & $(0,0,0) \leftarrow(0,0,5 / 2)$ \\
\hline $1^{\prime \prime}$ & 542.40 & $-128(10)$ & $?$ \\
\hline $1^{\prime}$ & 540.55 & $-65(20)$ & $(1,0,0) \leftarrow(1,0,1 / 2)$ \\
\hline $\mathbf{0}$ & 538.65 & 어) & origin \\
\hline $2^{\prime}$ & 531.20 & $260(10)$ & $\begin{array}{l}(0,1,1) \leftarrow\left(0,0,3 / 2^{ \pm}\right),(1,1,1) \leftarrow(1,0,1 / 2) \\
(0,2,0) \leftarrow(0,1,1 / 2)\end{array}$ \\
\hline 1 & 529.05 & $337(10)$ & $(0,1,0) \leftarrow(0,0, i / 2)$ \\
\hline$a_{1}$ & 526.85 & $416(15)$ & $(0,2,2) \leftarrow(0,0,5 / 2)$ \\
\hline$b_{1}$ & 524.40 & $504(15)$ & $(1,0,0) \leftarrow(0,0,1 / 2),(0,3,1) \leftarrow(0,1,3 / 2)$ \\
\hline $3^{\prime}$ & 521.95 & $594(15)$ & $\begin{array}{l}(0,2,2) \leftarrow(0,0,3 / 2 \pm),(0,3,3) \leftarrow(0,0,7 / 2) \\
(0,3,1) \leftarrow(0,1,1 / 2),(0,2,0) \leftarrow(1,0,1 / 2)\end{array}$ \\
\hline 2 & $\mathbf{5 1 9 . 7 4}$ & $675(10)$ & $(0,2,0) \leftarrow(0,0,1 / 2)$ \\
\hline$a_{p}$ & 517.79 & $748(15)$ & $(0,3,2) \leftarrow(0,0,5 / 2)$ \\
\hline b & $\$ 15.34$ & $840(15)$ & $(1,1,1) \leftarrow(0,0,1 / 2),(0,4,2) \leftarrow(0,1,3 / 2)$ \\
\hline$\ell^{\prime}$ & $\$ 13.0$ & $928(20)$ & $\begin{array}{l}(0,3,1) \leftarrow(0,0,3 / 2),(0,4,4) \leftarrow(0,0,7 / 2) \\
(0,4,0) \leftarrow(0,1,1 / 2),(1,3,1) \leftarrow(1,0,1 / 2)\end{array}$ \\
\hline 3 & $\mathbf{5 1 0 . 9 4}$ & $1007(10)$ & $(0,3,0) \leftarrow(0,0,1 / 2)$ \\
\hline$a_{3}$ & 508.39 & $1106(20)$ & $(0,4,2) \leftarrow(0,0,5 / 2)$ \\
\hline$b_{3}$ & 506.64 & $1173(15)$ & $(1,2,0) \leftarrow(0,0,1 / 2),(0,5,1) \leftarrow(0,1,3 / 2)$ \\
\hline $5^{\prime}$ & 504.30 & $1264(30)$ & $\begin{array}{l}(0,4,1) \leftarrow(0,0,3 / 2),(0,5,3) \leftarrow(0,0,7 / 2) \\
(0,5,1) \leftarrow(0,1,1 / 2),(1,4,0) \leftarrow(1,0,1 / 2)\end{array}$ \\
\hline 4 & 502.39 & $1340(10)$ & $(0,4,0) \leftarrow(0,0,1 / 2)$ \\
\hline
\end{tabular}


Figure (6.1) Photoelectron spectrum of $\mathrm{Si}_{3}$ - obtained using $3.493 \mathrm{eV}$ photon energy with the threshold photodetachmen! (ZEKE) spectrum superimposed on the inset.

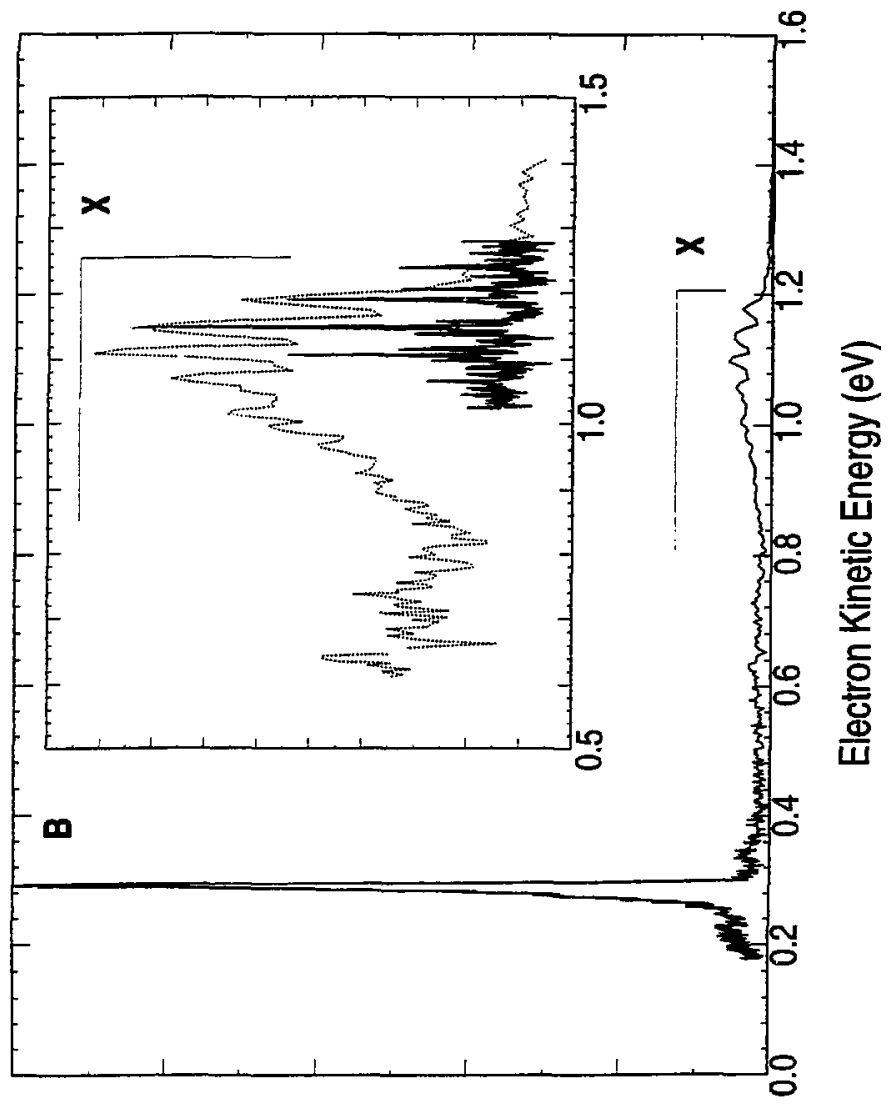


Figure (6.2) Threshold photodetachment spectrum of $\mathrm{Si}_{7}{ }^{-}$.

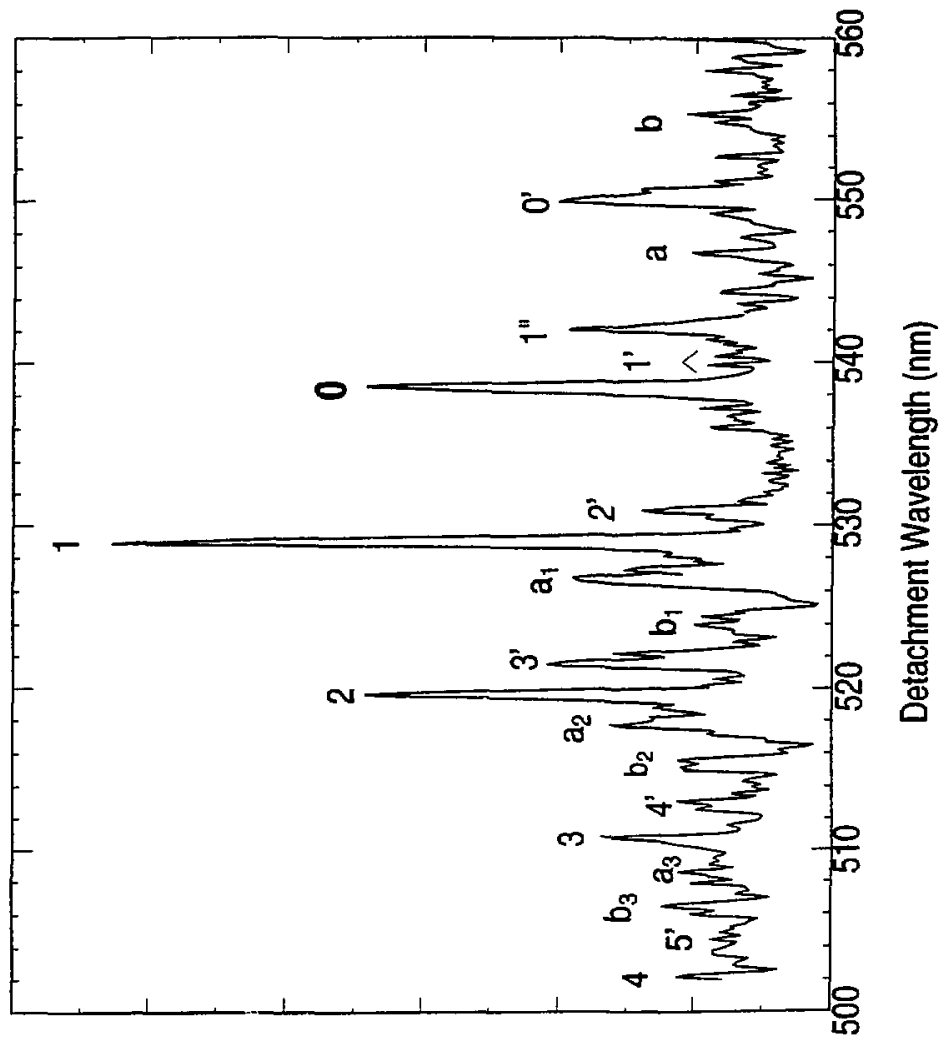


Figure (6.3) Experimental spectrum and three simulations (see text): Rigid anion, linear JahnTeller (LJT), and quadratic Jahn-Teller (QJT). All three simulations assume $337 \mathrm{~cm}^{-1}\left(e^{\prime}\right)$ and $500 \mathrm{~cm}^{-1}\left(a_{1}\right)$ neutral frequencies. The rigid anion simulation assumes the following anion frequencies (and temperatures): $v_{1}=560 \mathrm{~cm}^{-1}(500 \mathrm{~K}), v_{2}=385 \mathrm{~cm}^{-1}(450 \mathrm{~K}), v_{3}=270 \mathrm{~cm}^{-1}$ (350K). LJT assumes $120 \mathrm{~K}$ for $0,0,3 / 2$ level, $250 \mathrm{~K}$ for $0,0,5 / 2$ level , 350K for $0,1,1 / 2$ level (385 $\mathrm{cm}^{-1}$ above ground), 350K for $0,0,7 / 2$ level, and $400 \mathrm{~K}$ for the $(0,1,3 / 2),(1,0,1 / 2)$, and $(1,0,3 / 2)$ levels. QJT assumes $150 \mathrm{~K}$ for the $0,0,3 / 2$ levels (degeneracy broken), otherwise, temperatures are the same as LJT simulation.

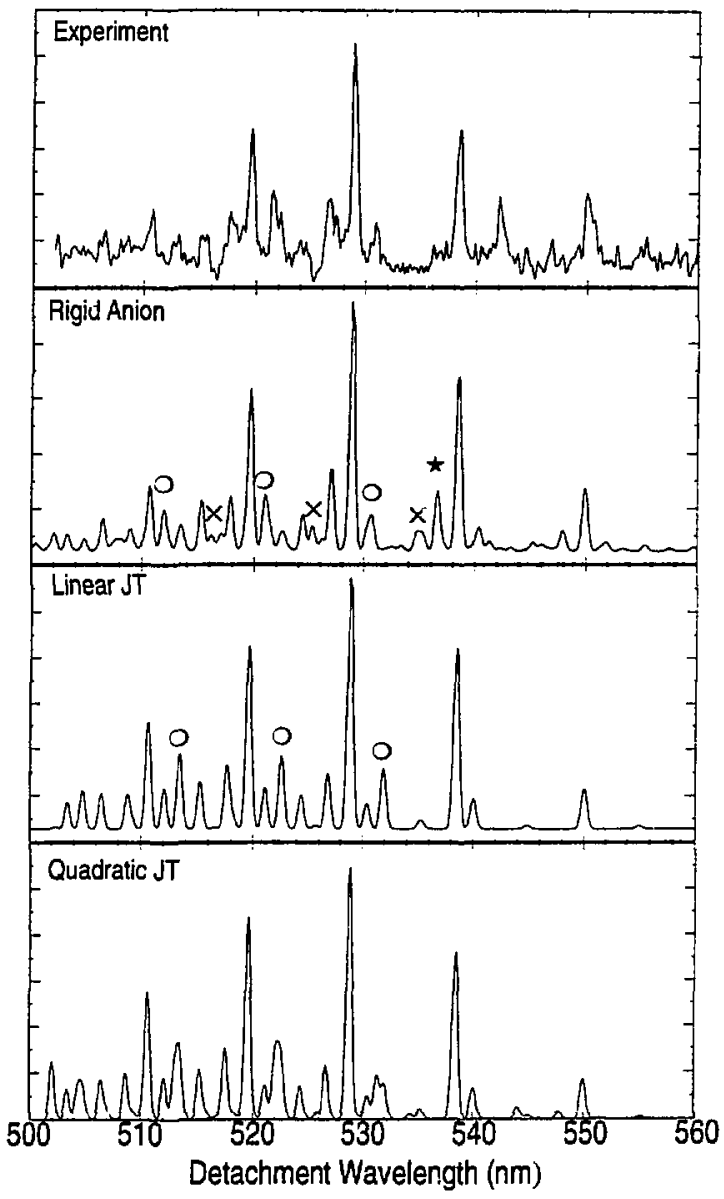


Figure (6.4) Schematic of $\mathrm{Si}_{3}$ and $\mathrm{Si}_{3}{ }^{-}$energy levels and some of the transitions proposed in the spectral assignment. See Table (6.1) for complete assignment.

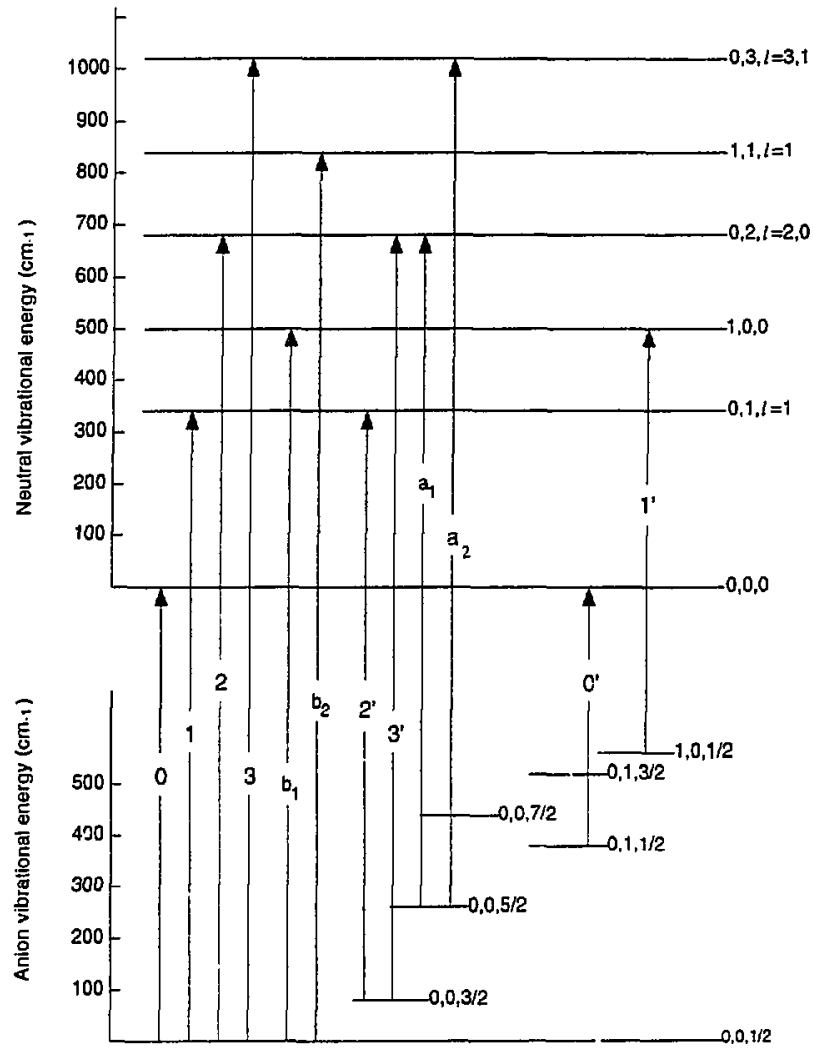


Figure (6.5) Simulation of band $\mathrm{X}$ in the $\mathrm{Si}_{3}^{-}$photoelectron spectum including contributions from (a) the ${ }^{3} A_{2}{ }^{\prime}+e^{*} \leftarrow{ }^{2} A_{1}$ transition, in which the experimental ZEKE spectrum is convoluted with the lower resolution of the photoelectron spectrometer, and (b) the ${ }^{1} A_{1}+e^{-} \leftarrow{ }^{2} A_{1}$ transition, which was simulated using $a b$ initio trequencies and geometries for the anion and neutral Origins for the two bands are indicated with anows; the origin for the singlet band is adjusted to best match the experiment.

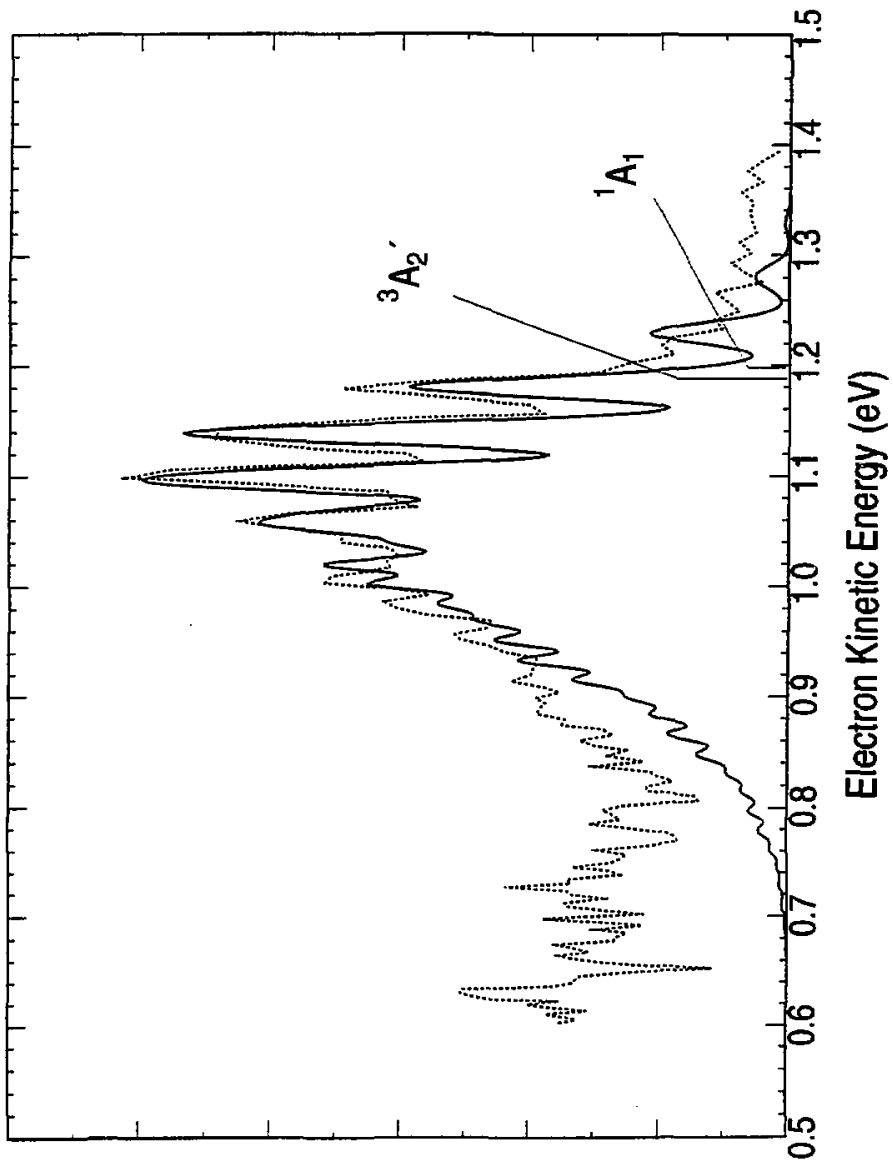




\section{Chapter 7. Study of $\mathrm{Si}_{4}$ and $\mathrm{Si}_{4}-$ using Threshold Photodetachment (ZEKE) Spectroscopy}

Abstract

The threshold photodetachment (ZEKE) spectrum of $\mathrm{Si}_{4}^{-}$is presented. Although, no transitions to the ground state of $\mathrm{Si}_{A}$ are observed, we obtain detailed information on the anion and several of the low-lying excited states of neutral $\mathrm{Si}_{4}$. The spectrum shows a long progression of wellresolved transitions between the $D_{2 h}{ }^{2} B_{2 g}$ mombus anion and $v_{2}$ vibrational levels of the first excited $D_{2 h}{ }^{3} B_{3 u}$ neutral. The length and spacing of the progression is consistent with ab initio calculations performed by Rohlfing and Raghavachan, [J. Chem. Phys. 96, 2114 (1992)] but some of the sequence bands observed within the progression are not. We also observe transitions to the $\mathrm{Si}_{4}{ }^{1} \mathrm{~B}_{3 u}$ state which is found at a lower excitation energy than predicted. The peturbed vibrational structure in this band is attributed to vibronic coupling to a nearby electronis state which is "dark" with respect to ZEKE spectroscopy. The ZEKE spectra are compared to the previously obtained photoelectron spectra of $\mathrm{Si}_{4}{ }^{-}$as well as ab initio calculations on $\mathrm{Si}_{4}{ }^{-}$ and $\mathrm{Si}_{4}$. 


\section{Introduction}

The study of small silicon clusters has been an area of interest because of their importance in astrophysics ${ }^{1}$ and CVD processes. ${ }^{2}$ These species are of considerable interest from a purely spectroscopic perspective as well, since $a b$ initio calculations $3,4,5$ predict dramatic changes in the geometry and bonding in silicon clusters as the number of atoms is varied. However, from an experimental viewpoint, the spectroscopy of silicon clusters is quite poorly characterized with the exception of the dimer, ${ }^{6}$ for which several rotationally-resolved electronic transitions have been observed. The development of laser vaporization cluster sources has facilitated photofragmentation. ${ }^{7}$ photoionization, ${ }^{8}$ and "reactive etching" studies, ${ }^{9}$ of the larger clusters, but these experiments have revealed little regarding specific cluster structures and bonding. Several absorption lines seen in in silicon vapors trapped in rare gas matrices were tentatively attributed to $\mathrm{Si}_{3}$ and $\mathrm{Si}_{4}$, but no analysis of these lines was pursued. ${ }^{10,11}$

There have been several gas phase spectroscopic studies of silicon clusters with three

or more atoms. With the exception of the emission spectroscopy study of Gole and coworkers, ${ }^{12}$ in which several transitions from two excited electronic states attributed to $\mathrm{Si}_{3}$ were observed, this work has involved negative ion photodetachment experiments on silicon cluster anions. ${ }^{13,14,15,16,17}$ While the resolution obtained in photodetachment experiments is typically lower than IR absorption techniques, they offer the advantage of mass-selection prior to spectroscopic investigation, thereby eliminating any ambiguity concerning species identification. Such experiments have also revealed a great deal about the electronic structure of metal clusters $^{18}$ and carbon clusters. ${ }^{19}$

Electronically resolved negative ion photoelectron spectra (PES) of $\mathrm{Si}_{n}^{-}$(n $=3$ through

12) have been obtained by Smalley and coworkers. ${ }^{14}$ Higher resolution PES have been 
measured by Ellison for $\mathrm{Si}_{2}{ }^{-15}$ and by our group for $\mathrm{Si}_{2}{ }^{-}-\mathrm{Si}_{4}-, 16,17$ These studies have shown that silicon clusters are rich with low-lying electronic states, and the higher resolution studies revealed vibrational structure associated with several of the neutral $\leftarrow$ anion electronic transitions. We have also studied $\mathrm{Si}_{2}{ }^{-}$at even higher resolution using threshold photodetachment (Zero electron kinetic energy, or ZEKE20) spectroscopy. ${ }^{16}$ The ZEKE spectrum exhibited fine structure due to spin-obit splitting both in the anion and neutral dimer, and this proved invaluable in sorting the low-lying electronic states in $\mathrm{Si}_{2}{ }^{-}$and $\mathrm{Si}_{2}$.

In the case of our $\mathrm{Si}_{3}-$ and $\mathrm{Si}_{4}-$ photoelectron spectra, even though vibrational structure is resolved in several of the bands, the assignment of the vibrational and electronic features is greatly facilitated by comparing the experimental spectra to the ab initio work of Paghavachan. Rohlfing, and co-workers. 3.4 For example, their calculations on $\mathrm{Si}_{4}$ and $\mathrm{Si}_{4}$ - predicted the ground siates to be the ${ }^{1} \mathrm{~A}_{\mathrm{g}}$ and the ${ }^{2} \mathrm{~B}_{2 \mathrm{~g}}$ states, respectively, both of which have planar hombus $\left(D_{2 n}\right)$ structures. Based on the calculated geometries, which are quite similas, one would expect to see a short progression in the $v_{2}$ mode of $\mathrm{Si}_{4}$ with a frequency of $357 \mathrm{~cm}^{-1}$. This is consistent with the experimental spectra, ${ }^{17}$ shown in Figure (7.1), thereby supporting the ab initio geometries.

Rohlfing and Raghavachari ${ }^{4}$ have also investigated the excited states of $\mathrm{Si}_{4}$ with a thombus (or near hombus) equilibrium geometry. They determined that at least six electronic states accessible by detachment of the anion ground state $\left({ }^{2} \mathrm{~B}_{2 \mathrm{~g}}\right)$ lie within $2 \mathrm{eV}$ of the neutral ground state, and assigned the three excited state bands in our spectrum (bands A, B, ano $\mathrm{C}^{17}$ ) to transitions to the ${ }^{3} \mathrm{~B}_{3 u},{ }^{3} \mathrm{~B}_{\mathrm{g}}$, and ${ }^{3} \mathrm{~B}_{1 \mathrm{u}}$ states of $\mathrm{Si}_{4}$. They also raised the possibility o. tnat one of the bands (band B) consisted of overlapping ansitions other low lying excited states of $\mathrm{Si}_{4}$. The band assignments according to Ref. 4 are indicated in Figure (7.1).

The partially resolved vibrational teatures in Figure (7.1) suggest that a higher resolution photodetachment spectrum will reveal considerably more structure. This is the motivation for 
the work presented here, in which we report the results of threshold photodetachment (ZEKE) spectroscopy on $\mathrm{Si}_{4}{ }^{-}$. In these experiments, mass-selected anions are photodetached with a tunable laser, and only those electrons with nearly zero kinetic energy are detected. The resolution of our ZEKE spectrometer is around $3 \mathrm{~cm}^{-1}$, considerably better than the photoelectron spectrometer used for Figure $(7.1)\left(80-100 \mathrm{~cm}^{-1}\right)$.

Threshold photodetachment spectroscopy is most powerful when used in tandem with photoelectron spectroscopy. While all neutral $\leftarrow$ anion electronic transitions involving removal of a single electron can be seen in the photoelectron spectrum of an anion. this is not so for the ZEKE spectrum. According to the Wigner ${ }^{21}$ threshold law, the photodetachment cross section near the threshold for a neutral $\leftarrow$ anion transition goes as

$$
\sigma \propto\left(E_{\text {photon }}-E_{\text {threshold }}\right)^{l+1 / 2}
$$

where $\ell$ is the photoelectron angular momentum. Fo: $\ell=0$ (s-wave) detachment, the cross section rises sharply above threshold, but for $\ell \geq 1$ (p-wave, etc.) the cross section is very small near threshold. Hence, ZEKE spectroscopy of anions is sensitive only to s-wave detachment. While sometimes inconvenient, this feature is often useful in assigning neutral $\leftarrow$ anion electronic transitions, since s-wave detachment can only result from removal of an electron from particular orbitals. ${ }^{22}$ This type of analysis was used in our study of $\mathrm{Si}_{2}{ }^{-16}$ as well as in the work presented here.

The $\mathrm{Si}_{4}-$ threshold photodetachment spectrum shows well resolved bands involving transitions between the $\mathrm{Si}_{4}-{ }^{2} \mathrm{~B}_{2 g}$ state and two excited electronic states of neutral $\mathrm{Si}_{4}$. The lower energy band, assigned to the transition to the $\mathrm{Si}_{4}{ }^{3} \mathrm{~B}_{3 \mathrm{u}}$ state, is particularly clean, and from it we obtain several vibrational frequencies for the anion and neutral which are in partial agreement with the $a b$ initio results of Rohlfing and Raghavachari.4 The higher energy band 
appears to be strongly pertubed, most likely because of vibronic coupling to an overlapping $\mathrm{Si}_{4}$ excited state which is "dark" with respect to ZEKE spectroscopy. The nature of the electronic states that are involved in this band is discussed in light of the ab initio calculations.

\section{Experimental}

Figure (7.2) shows a schematic top view of the threshold photodetachment apparatus. It is described in detail elsewhere, ${ }^{23}$ but the basic operation is as follows. Cold silicon clusters (anions, cations, and neutrals) are generated in a laser vaporization/puised molecular beam

source similar to that developed by Smalley. ${ }^{24}$ Helium is used as the carrier gas, typically with a backing pressure between 50 and 80 psi. Use of a piezoelectric valve 25 rather than the solenoid-type molecular beam valve (General Valve) used in previous cluster studies was found to greatly enhance cluster cooling. Laser vaporization is achieved by focussing a $2 \mathrm{~mJ}, 532 \mathrm{~nm}$ pulse from a frequency-doubled Nd:YAG laser ( $20 \mathrm{~Hz}$ repetition rate) onto a rotating, translating silicon rod. The negative ions that pass through a $2 \mathrm{~mm}$ diameter skimmer are collinearly accelerated to $+\mathrm{keV}$. Mass selection is achieved by a $1 \mathrm{~m}$ long beam-modulated time-of-flight mass spectrometer. The mass separated ions then enter the detector region where they are photodetached by a (pulsed) excimer-pumped dye laser. The dyes used for photodetachment of $\mathrm{Si}_{4}{ }^{-}$were Coumarin 503 (479-553 nm), Exalite 416 (402-427 nm). DPS (399-415 nm), BBQ (367-405 nm). QUI (368-402 nm), DMQ (346-377 nm), pTp (332-350), and, doubled with a $\beta-$ barium borate (BBO) crystal, Rhodamine 640 (310-332 nm) and Rhodamine 590 (287-300 nm).

Threshold photodetachment spectra are obtained by adapting the ZEKE spectroscopy method developed by Müller-Dethlefs at $a l^{20}$ to negative ion photodetachment. In this experiment, the $\mathrm{Si}_{4}-$ is photodetached in the region marked by the $\times$ on Figure (7.2). The photoelectrons are extracted with a delayed, pulsed field. This allows the higher energy 
photoelectrons to spitially separate from the threshold electrons, which have nearly zero kinetic energy. The energetic electrons that scatter away from the ion beam axis are discriminated against by apertures located in the detector region. Those that scatter along the ion beam arrive at the detector at different times from the threshold electrons and are discriminated against by gated integration of the threshold electrons. This combination of spatial and temporal discrimination yields an electron energy resolution of $3 \mathrm{~cm}^{-1}$.

As an altemative mode of operation, the photoelectrons can be extracted immediatedly upon photodetachment. This results in spectral features that are $150 \mathrm{~cm}^{-1}$ wide but have considerably higher intensity. While no high resolution information can be obtained in this mode of operation, it is useful as a first order method of locating the s-wave photodetachment thresholds, especially when the photodetachment signal is too small to obtain ZEKE spectra.

Total photodetachment cross section scans were also performed for $\mathrm{Si}_{4}{ }^{-}$to determine whether any high-lying (autodetaching or bound) excited anion states existed for $\mathrm{Si}_{4}-$ as they did for $\mathrm{C}_{6}-{ }^{26}$ No such states were found, and this experiment will not be discussed further.

\section{Results}

The spectrum of $\mathrm{Si}_{4}-$ obtained on the threshold photodetachment apparatus is shown superimposed on the photoelectron spectrum of $\mathrm{Si}_{4}{ }^{-17}$ in Figure (7.3). In the ZEKE mode of operation, we saw the two bands labelled $A^{\prime}$ and $B^{\prime}$. Band $A^{\prime}$ corresponds well with band $A$ seen in the PES, but band $B^{\prime}$ falls primarily on the rising edge of band $B$ in the PES. No structure is seen in the ZEKE spectrum for the remainder of the energy range sparined by the PES band $B$. Band $C^{\prime}$, which corresponds to band $C$ in the PES, could only be observed in the partially discriminated mode of operation due to its low signal intensity; this is why the features in this band are broader. No signal corresponding to band $X$ (the transition to the $\mathrm{Si}_{4}$ ground state) in 
the PES could be observed in either the ZEKE or partially discriminated mode. Hence, the three observed bands all involve transitions to excited states of $\mathrm{Si}_{4}$. The bands will now be examined in greater detail.

\section{A. Band $A^{\prime}$}

Band $A^{\prime}$ in the threshold photodetachment spectum shows a progression of up to 14 peaks which corresponds to the partially resolved vibrational structure seen previously in the $\mathrm{Si}_{4}-$ PES. ${ }^{17}$ The PES had to be shifted along the abscissa by $-0.03 \mathrm{eV}$ in order to fully align the PES and ZEKE progressions. Assuming this shift applies to the entire photoelectron spectrum, the electron affinity of $\mathrm{Si}_{4}$ previously obtained from the PES should be changed slightly to $2.17 \pm$ $0.01 \mathrm{eV}$. Figure (7.4) shows a finer-step, signal averaged scan of this band. Clearly, each member of this progression consists of groups of smaller peaks found to either side of a single, more intense peak. The intense peak in each group is labelled by number and the positions and spacings of these peaks are listed in Table (7.1). The average peak spacing is $312 \mathrm{~cm}^{-1}$.

Figure (7.5) shows a more expanded scale scan of the two groups of peaks around peaks 3 and 4 . The more intense side peaks, labelled $a_{n}$ through $e_{n}$, shade to higher photon energy relative to the most intense peak (peak n) in each group, while the less intense side peaks, labelled $t_{n}$ and $g_{n}$ shade to lower photon energy. The positions and energies of these side progressions are listed in Table (7.2) for the two groups shown in Figure (7.4). All of the main and side peaks are between 16 and $20 \mathrm{~cm}^{-1}$ wide (FWHM). We note that this patiern of peaks occurs, with some intensity variation, for every group of peaks in band $A^{\prime}$.

The intensities of peaks $a_{n}$ through $g_{n}$ are dependent on source conditions. When we produced ions using a solenoid-type pulsed molecular beam valve (i.e., higher temperature) instead of the piezoelectric valve, $a_{n}$ and $b_{n}$ were as intense as the main peak $n$ while $c_{n}$ through 
$e_{n}$ remained unresolved in a broad signal with half the intensity of the $n-a_{n}-b_{n}$ triplet. The intensities of peaks $g_{n}$ and $f_{n}$ are also dependent on source conditions, although they never exceeded one half the $n$ peak intensity. This source condition dependence indicates that these peaks are due to transitions from vibrationally excited levèls of the anion, or, "hot-band" transitions. The assignments of these peaks are addressed in the following section.

At first glance, peaks $a_{n}$ through $e_{n}$ appear to belong to one progression with a spacing of $-25 \mathrm{~cm}^{-1}$. However, the intensity distribution of these peaks indicate otherwise. Peak $b_{n}$ is typically of comparabie intensity to $a_{n}$, whereas peak $c_{n}$ is quite small compared to $b_{n}$, if it can be resolved at all. Therefore, peaks $a_{n}$ through $e_{n}$ appear to involve two vibrational modes. For instance, $b_{n}$ has an average spacing from $n$ of $53 \mathrm{~cm}^{-1}$, and $d_{n}$ is $51 \mathrm{~cm}^{-1}$ from. $b_{n}$ on average, and for several of the main spectral features, an $\mathbf{e}_{n}$ peak can also be seen approximately 50 $\mathrm{cm}^{-1}$ to the blue of peak $d_{n}$. The $a_{n}$ peaks are spaced approximately $30 \mathrm{~cm}^{-1}$ from peak $n$ for low $n$, but this spacing decreases down to $26 \mathrm{~cm}^{-1}$ to higher values of $n$. The same effect is seen for the energy difference between $c_{n}$ and $b_{n}$ in those cases where $c_{n}$ is well enough resolved. Therefore, we betieve that $n, b_{n}, d_{n}$ and $e_{n}$ belong to a $50 \mathrm{~cm}^{-1}$ progression in one mode, and that $n, a_{n}$, and $b_{n}, c_{n}$ represent shorter $30 \mathrm{~cm}^{-1}$ progressions iri a second mode.

The side peaks found to the red of the main peak are fewer and less intense. Th? $t_{n}$ peaks are found approximately $25 \pm 5 \mathrm{~cm}^{-1}$ to the red of peak $n$. In some cases, such as for $n=$ $5, f_{n}$ appears to be hardly resolved from the main peak. The $g_{n}$ peaks are found approximately $53=5 \mathrm{~cm}^{-1}$ to the red of the main peaks $n$. Figure $(7.4)$ shows that the intensities of the $g_{n}$ peaks relative to the main peaks is not constant throughout the progression; for $n=0-3$, the $g_{n}$ peaks are fairly prominent, but peaks $g_{5}$ and $g_{6}$ cannot be discemed from the noise. For $n>8$, the $g_{n}$ peaks again become apparent. 


\section{B. Band $B^{\prime}$}

As can be seen from the ZEKE portion of the spectrum shown in Figure (7.3), band $B^{\prime}$ is much less intense than band $A^{\prime}$. In addition, the progression is much shorter. Note that while band $A^{\prime}$ closely matches band $A$ in the photoelectron spectrum, band $B^{\prime}$ covers a much smaller energy range than band $B$ in the PES. No structure was seen between band $B^{\prime}$ and $300 \mathrm{~nm}$, even using the partially discriminated cross section mode of the apparatus. Figure (7.6) shows band $B^{\prime}$ on an expanded scale. Because the signal was so much less for this band, the signalto-noise ratio is not as good as for band $A^{\prime}$.

Again, the members of the progression in this band apnear as groups of peaks, but the profiles and relative intensities of groups exhibit irregularity. The first three members of the progression are qualitatively similar in profile to those found in the band $A^{\prime}$ in that the groups are dominated by a central intense peak, labelled $n^{\prime}$, as well as fairly intense side peaks, $a_{n^{\prime}}$ and $b_{n^{\prime}}$, found to higher photon energy and a less intense side peak, $g_{n^{\prime}}$, at lower photon energy. The central peaks $n^{\prime}$ in the first three groups seem to form a short, $300 \pm 6 \mathrm{~cm}^{-1}$ progression. However, the profiles of the fourth and fifth groups are quite different. There is no peak at the correct position in the fourth group to be a part of the $300 \mathrm{~cm}^{-1}$ progression, although the average position of the two intense peaks labelled $a_{3^{\prime}}$ and $f_{3^{\prime}}$ is just where peak $3^{\prime}$ would be expected. In the fifth group, peak 4', while apparently part of the main $300 \mathrm{~cm}^{-1}$ progression, is noticeably less intense than the peaks to either side. Peak $5^{\prime}$ can barely be discerned from the nojse. Peak positions and spacings for band $B^{\prime}$ can be found in Table (7.3).

\section{Band $\mathrm{C}^{\prime}$}

We were restricted to examining this band using the partially discriminated cross section mode of the apparatus due to very low photoelectron signal. The low signal is due to snalf 
photodetachment cross section near threshold for this state and lower photodetachment laser power.

The broad features of band $C^{\prime}$ shown in Figure (7.3) are roughly $200 \mathrm{~cm}^{-1}$ wide and are separated by approximately $430 \mathrm{~cm}^{-1}$ peak to peak. The broad peaks are not perfectly symmetric; there are probably many sequence bands (as were seen for the higher resolution sections of the spectrum) and/or combination features contributing to the profile of these features.

\section{Analysis and Discussion}

According to Rohifing and Raghavachari, ${ }^{4}$ the ${ }^{2} \mathrm{~B}_{2 g}$ anion ground state has the valence orbital configuration ...( $\left(b_{3 g}\right)^{2}\left(b_{3 u}\right)^{2}\left(a_{g}\right)^{2}\left(b_{1 u}\right)^{2}\left(b_{2 g}\right)^{1}$. Electrons removed from either the $b_{1 u}$ or the $b_{3 u}$ orbitals can depart via s-wave photodetachment, while this is not the case for the $b_{2 g}$ or $a_{g}$ orbitals. 22 Therefore, according the assignment of the $\mathrm{Si}_{4}-\mathrm{PES}$ in Ref. (4), [see band labels on Figure (7.1)] we would expect to see bands $A$ and $C$ in the ZEKE spectrum, which wa do, since these states result irom the removal of an electron from the $b_{1 u}$ and $b_{3 u}$ orbitals, respectively. Band $X$ in the PES is not seen in the ZEKE spectrum, which is consistent with its assignment to the ${ }^{9} A_{g}+e^{-} \leftarrow{ }^{2} B_{2 g}$ transition. This transition involves removing the $b_{2 g}$ electron, which would depart as a p-wave. Band $B$ seen in the PES was assigned to the ${ }^{3} B_{g}+e^{-} \leftarrow{ }^{2} B_{2 g}$ transition, ${ }^{4}$ corresponding to removal of an $\mathrm{a}_{g}$ electron. This transition, therefore, should not be apparent in the ZEKE spectrum, but we do observe band B' which corresponds to the rising edge of band $B$ in the PES. As discussed below, we believe band B is due to overlapping electronic transitions, one of which can occur by s-wave detachment. We now consider all three bands in the ZEKE spectrum in more detail. 


\section{A. Band $A^{\prime}$}

The energy of band $A^{\prime}$ and the fact that we observe it in the ZEK.E spectrum is consistent with the $a b$ initio assignment of this band to the ${ }^{3} \mathrm{~B}_{3 u}+e^{-} \leftarrow{ }^{2} \mathrm{~B}_{2 g}$ transition.4 The progression of the central peaks [peaks $n$ in Figure $(7.4)$ can be fit with the harmonic frequency $\omega_{e}=312 \pm 1 \mathrm{~cm}^{-1}$ and a very small anharmonic constant $\omega_{\mathrm{e}} x_{\mathrm{e}}=0.3 \pm 0.1 \mathrm{~cm}^{-1}$. The harmonic frequency is very close to the $a b$ initio value of $306 \mathrm{~cm}^{-1}$ for the $\mathrm{v}_{2}\left(\mathrm{a}_{\mathrm{g}}\right)$ mode in the $\mathrm{Si}_{4}{ }^{3} \mathrm{~B}_{34}$ state. Moreover, the ab initio geometries for $\mathrm{Si}_{4}{ }^{-}$and the ${ }^{3} \mathrm{~B}_{34}$ state of $\mathrm{Si}_{4}$, which are shown in Figure (7.7) along with the $v_{2}$ and $v_{1}\left(a_{g}\right)$ nomal modes of the ${ }^{3} B_{3 u}$ neutral (the force constants for determining the normal modes were obtained from our own ab initio casculations on $\mathrm{Si}_{4}$ ) suggest a large displacement along the $\mathrm{v}_{2}$ normal coordinate upon photodetachment, but almost no displacement along the higher frequency $v_{1}$ nomal coordinate. Hence, the $v_{2}$ mode should be the only active vibration in the ZEKE spectrum. We therefore assign peaks $n$ to the $2_{0}^{n}$ progression.

Although the assignment of the $2_{0}^{n}$ progression is straightforward, locating the origin is somewhat more ambiguous. Peak 0 , located at $415.27 \mathrm{~nm}$, appears to be the lowest energy peak in the $312 \mathrm{~cm}^{-1}$ progression, but one could argue that there is a small peak buried under noise at $312 \mathrm{~cm}^{-1}$ lower in energy $(420.68 \mathrm{~nm})$, or even $624 \mathrm{~cm}^{-1}$ to the red of peak $0(426.28$ $\mathrm{nm}$ ) where no detectable electron signal was seen.

We attempted to determine the location of the origin and the actual normal mode displacement by performing a single mode $\left(v_{2}\right)$ Franck-Condon simulation of the spectrum. Simulations performed assuming the origin was at $415.27 \mathrm{~nm}$ (with $\Delta Q$ of $0.219 \pm 0.008$ $\left.\not(\mathrm{amu})^{1 / 2}\right), 420.68 \mathrm{~nm}\left(\Delta \mathrm{Q}-0.235 \pm 0.008 \not h(\mathrm{amu})^{1 / 2}\right)$ or $426.28 \mathrm{~nm}(\Delta \mathrm{Q} 0.259=0.008$ $\dot{A}(\mathrm{amu})^{1 / 2}$ ) all satisfactorily matched the expenimental progression. However, based on the ab initio geometries and force constants, we can calculate the $v_{2}$ nomal mode displacement 
between the anion and neutral. We find this to be $0.208 \mathrm{~A}$ (amu $)^{1 / 2}$, which is in best agreement with the normal coordinate displacement assumed for the simulation with the origin at $\mathbf{4 1 5 . 2 7}$ $\mathrm{nm}$. We therefore take this to be the origin. The excitation energy of the ${ }^{3} \mathrm{~B}_{3 u}$ state is then $0.815 \pm 0.010 \mathrm{eV}$ [listed in Table (7.4)] which is in good agreement with the $0.85 \mathrm{eV}$ excitation energy calculated by Rohlfing and Raghavachari. ${ }^{4}$

We next consider the set of peaks $g_{n}$, each of which is located $53 \mathrm{~cm}^{-1}$ to the red of the corresponding central peak $\mathbf{n}$. The bimodal intensity distribution of these peaks, passing through a minimum at $n=5-6$, ran be explained by assigning them to a "hot-band" progression in the the $v_{2}$ mode originating from the $v_{2}=1$ level of the anion, i.e. a $2_{1}^{n+1}$ progression. The intensity minimum can be qualtatively understood by referring to Figure $(7.8)$, which shows anion and neutral harmonic potential energy curves displaced along the $Q_{2}$ coordinate by $0.219 \AA$ (arnu $)^{1 / 2}$, the displacement that best reproduced the iniensity distribution of the main progression (peaks n). This displacement is such that a node in the neutral $v_{2}=6$ wave function coincides with one of the the maxima of the anion $v_{2}=1$ wave function, causing the overlap to be close to zero. Based on this assignment, the $v_{2}$ frequency in the anion is $365 \mathrm{~cm}^{-1}$, quite close to the $a b$ initio frequency of $383 \mathrm{~cm}^{-1}, 4$ The intensity distribution of peaks $n$ and $g_{n}$ can be satisfactorily reproduced in a one-dimensional simulation (not shown) where the vibrational temperature of the anions is assumed to be $200 \mathrm{~K}$.

The large geometry change and faitly large frequency change in the $v_{2}$ mode between the anion and neutral can be explained by considering the orital from which the electron is removed in the neutral $\leftarrow$ anion transition. The $b_{1 u}$ orbital is formed by the (in-plane) $p_{2}$ orbitals [see Figure (7.7)] which effectively bonds the silicon atoms labelled 1 and 3. Removal of an electron from this orbital will weaken this bond, resulting in the longer bond distance and lower $v_{2}$ frequency in the neutral, since this mode primarily involves the stretching of $r_{b}$. 
We next consider the other vibrational features in band $A^{\prime}$, peaks $a_{n}-f_{n}$. As mentioned earlier, these peaks originate from vibrationally excited levels in the anion, and we assign them to sequence bands in modes other than the $v_{2}$ mode, that is, $2_{0}^{n} m_{1}^{1}, 2_{0}^{n} m_{2}^{2}$, etc. transitions. There are five other normal modes of $\mathrm{Si}_{4} / \mathrm{Si}_{4}-$ that could be contributing to this structure. However, using a vibrational temperature of $200 \mathrm{~K}$ as a guideline, significant population is expected only in the four lowest frequency modes of the anion. In order of increasing frequency, these are calculated to be the $v_{6}\left(165 \mathrm{~cm}^{-1}\right), v_{5}\left(295 \mathrm{~cm}^{-1}\right), v_{2}\left(383 \mathrm{~cm}^{-1}\right)$, and $v_{3}\left(390 \mathrm{~cm}^{-1}\right)$ modes. The normal modes are shown in Figure (7.7).

The $v_{2}$ mode has atready been accounted for. The $f_{n}$ peaks, each of which lies $25 \mathrm{~cm}^{-1}$ to the blue of the main peak $n$, are of comparable intensity to the most intense $g_{n}$ peaks, and likely onginate from the $v=1$ level of a mode close in frequency to the $v_{2}$ mode. According to the $a b$ initio results, the most likely candidate is the $v_{3}$ mode, and we therefore assign peaks $f_{n}$ to the $2_{0}^{n} 3_{1}^{1}$ progression. Although we cannot directly extract the $v_{3}$ anion and neutral frequencies from the peak positions, they do yield the difference between the anion and neutral frequencies, and show that the $v_{3}$ mode is $25 \mathrm{~cm}^{-1}$ lower in the neutral. This is consistent with the ab initio results, which give $v_{3}=367 \mathrm{~cm}^{-1}$ in the neutral, $23 \mathrm{~cm}^{-1}$ less than the calculated anion frequency. As discussed eartier, peaks $a_{n}-e_{n}$ to the red of peak $n$ appear to be sequence bands composed of two overlapping progressions. The presence of sequence bands to the red of the central peak means that the vibrational modes involved have higher frequencies in the neutral than in the anion. This is at odds with the ab initio results, ${ }^{4}$ for which all of the frequencies for the $\mathrm{Si}_{4}{ }^{3} \mathrm{~B}_{34}$ state are lower than in the anion. Since the leading members of these progressions, peaks $a_{n}$ and $b_{n}$, are quite intense, these progressions most likely involve the two lowest frequencies of the anion, the $v_{5}$ and $v_{6}$ modes. From the positions of peaks $a_{n}$ and $b_{n}$ relative to peak $n$, the frequencies of one of these modes is $27 \mathrm{~cm}^{-1}$ higher in the neutral than in the anion, while the other is $52 \mathrm{~cm}^{-1}$ higher in the neutral. In contrast, the $a b$ initio 
calculations predict the $v_{5}$ and $v_{6}$ frequencies to be higher in the anion by $118 \mathrm{~cm}^{-1}$ and $8 \mathrm{~cm}^{-1}$, respectively.

We therefore have two possible assignments for these peaks. In one assignment, peak $a_{n}$ is the $2{ }_{0}^{n} 6{ }_{1}^{1}$ transition and peak $b_{n}$ is primarily the $2_{0}^{n} 5_{1}^{1}$ transition (with some contribution from the $2{ }_{0}^{n} 6_{2}^{2}$ transition). These assignments and those of peaks $c_{n} \cdot e_{n}$ are listed in Table (7.2). In the other assignment, $v_{5}$ and $v_{6}$ are switched. In order to better choose between these assignments, we can simulate the spectrum using the four modes considered above $\left(v_{2}, v_{3}, v_{5}\right.$, $v_{6}$ ). We do this first for the assignment in Table (7.2). For the $v_{5}$ and $v_{6}$ modes, we need to assume values for the neutral frequencies, and these will fix the anion frequencies. If we choose the $a b$ initio values for the neutral frequencies, the anion frequencies are those given in Table (7.5). We obtain the simulation shown in Figure (7.9) using $200 \mathrm{~K}$ as the vibrational temperature for the $v_{2}$ mode and slightly different vibrational temperatures for the other three modes [listed in Table (7.5)]. The temperatures were fit paying closest attention to peak groups with $n=3$ and 4 , since our data is best for these [see inset, Figure (7.9)].

The reasonable fit between the simulated and experimental spectrum using vibrational temperatures close to $200 \mathrm{~K}$ for all four modes offers indirect support both for the assignment of peaks $a_{n}-e_{n}$ and for using the ab initio frequencies for the neutral. While different modes have different cooling efficiencies in a free jet expansion, we would expect the temperatures of the remaining modes to be in the neighborhood of $200 \mathrm{~K}$, the appropriate temperature for the $v_{2}$ mode, particularly if they have similar or lower frequencies. If we use the alternate assignment proposed above, with the $v_{5}$ and $v_{6}$ modes switched from Table (7.2), and use the $a b$ initio values for the neutral frequencies, then the anion frequencies are $v_{5}=150 \mathrm{~cm}^{-1}$ and $v_{6}=107$ $\mathrm{cm}^{-1}$, and the required vibrational temperatures are $380 \mathrm{~K}$ and $107 \mathrm{~K}$, respectively [these values are included parenthetically in Table (7.5)]. This is a noticeably larger deviation from $200 \mathrm{~K}$ than 
the values obtained using the former assignment, and makes this assignment somewhat less desirable.

Another option is to use the $a b$ initio $v_{5}$ and $v_{6}$ frequencies for the anion [see Table (7.5) \}, and fix the neutral frequencies accordingly. Using the peak assignments in Table (7.2), we require vibrational temperatures of $650 \mathrm{~K}$ and $450 \mathrm{~K}$ for the $v_{5}$ and $v_{6}$ modes, respectively. These temperatures for the lowest frequency modes seem unreasonably large compared to the $200 \mathrm{~K}$ temperature for the $v_{2}$ mode. A similiarly large deviation is obtained using the alternate assignment assuming the ab initio anion frequencies.

Overall, the peak assignments in Table (7.2) and the frequencies listed in Table (7.5) seem the most reasonable of all the possibilities considered. The smalt frequency change in the $v_{6}$ mode upon photodetachment to the ${ }^{3} \mathrm{~B}_{3 u}$ state is sensible as it is an out-of-plane vibration whereas the $b_{1 u}$ orbital is primarily in the plane of the molecule. The incr ase in the $v_{5}$ frequency upon photodetachment is more difficult to rationalize on the basis of molecular orbital considerations.

The spectra support the ab initio geornetries for $\mathrm{Si}_{4}{ }^{-}$and the $\mathrm{Si}_{4}$ excited state, as well as the ab initio frequencies for the anion and neutral $v_{2}$ and $v_{3}$ modes. However, the spectra are inconsistent with the $a b$ initio $v_{5}$ and $v_{6}$ frequencies. The $v_{5}$ frequency for the anion is particularly suspect, since it lies well above the predicted neutral frequency, whereas our spectrum shows it should be less than the neutral frequency. Moreover, a high vibrational temperature for the $v_{5}$ mode is always needed to simulate the spectrum if the ab initio value is used. It is possible that the the large discrepancy between the $a b$ initio $\mathrm{Si}_{4} / \mathrm{Si}_{4}-v_{5}$ frequency difference and the observed frequency difference is due to an incorrect assignment of the $b_{n}$ peaks. For instance, $b_{n}$ could be due to a combination band; if $b_{n}$ were due to the $2_{0}^{n-1} 5_{0}^{2}$ transition, its position would be in fairly good agreement with the calculated $v_{5}$ frequency. However, were this the case, peak $b_{0}$ (found $50 \mathrm{~cm}^{-1}$ from the origin) would not appear in the 
spectrum. Also, the intensity of the $b_{n}$ peaks is too high for a $\Delta v=2$ transition in a non-totally symmetric mode. Thus, we believe our assignment of the $b_{n}$ peaks to sequence band transitions is correct. In any case, independent measurements of either the anion or neutral $v_{5}$ and $v_{6}$ frequencies would result in a more complete understanding of the ZEKE spectrum.

\section{B. Band B'}

Rohlfing and Raghavachari assigned band $B$ shown on the PES [Figure (7.1)] to the ${ }^{3} \mathrm{~B}_{\mathrm{g}}$ $+\mathrm{e}^{-} \leftarrow{ }^{2} \mathrm{~B}_{2 g}$ iransition, ${ }^{4}$ where the ${ }^{3} \mathrm{~B}_{\mathrm{g}}$ state has a $\mathrm{C}_{2 h}$ structure resulting from a slight distortion of the ${ }^{3} \mathrm{~B}_{2 g}\left(\mathrm{D}_{2 h}\right)$ state. They pointed out that the irregular spacings of the band may be due to contributions from the nearby ${ }^{3} B_{1 g},{ }^{1} B_{2 g}$ and ${ }^{1} B_{3 u}$ states. However, only the ${ }^{1} B_{3 u}$ state is accessible from the anion by s-wave detachment. This suggests that band $B^{\prime}$ in the ZEKE spectrum and the leading edge of band $B$ in the photoelectron spectrum are due to the ${ }^{1} B_{3 u}+e^{-}$ $\leftarrow{ }^{2} B_{2 g}$ transition. The ${ }^{1} B_{3 u}$ state is the singlet counterpart of the ${ }^{3} B_{3 u}$ state responsible for band $A^{\prime}$, so the apparent origin of band $B^{\prime}$ at $349.90 \mathrm{~nm}$ implies a singlet-triplet splitting of 0.558 eV for the $\mathrm{B}_{3 \mathrm{u}}$ state. This is lower than the calculated splitting, $1.04 \mathrm{eV}$, but the disagreement is not so unreasonable considering the degree of difficulty in accurately calculating singlet-triplet splittings in complex molecules.

The profile of band $\mathbf{B}^{\prime}$ is very different from band $A^{\prime}$, with the intensity of band $\mathbf{B}^{\prime}$ falling off after only a few peaks. Moreover, the vibrational structure in band $\mathbf{B}^{\prime}$ is irregular. As mentioned in Sec. III, the first three groups of peaks in this band [Figure (7.5)] are similar to the peak groups in the band $A^{\prime}$. The spacing of the central peaks $0^{\prime}-2^{\prime}$ is $300 \pm 8 \mathrm{~cm}^{-1}$, and the faily intense sequence bands occuring to the red of these features $\left(g_{n}\right)$ are spaced such that the anion frequency is approximately $365 \pm 5 \mathrm{~cm}^{-1}$. Thus, the $\mathrm{n}^{\prime}$ peaks appear to be a progression in the $v_{2}$ mode of the neutral ${ }^{1} B_{1 u}$ state, with a frequency $12 \mathrm{~cm}^{-1}$ lower than the $v_{2}$ 
mode in the ${ }^{3} \mathrm{~B}_{1 u}$ state. The fourth group of peaks is very different; it is an intense doublet separated by approximately $38 \mathrm{~cm}^{-1}$. These peaks, $f_{3^{\prime}}$ and $a_{3^{\prime}}$, are separated by $279 \mathrm{~cm}^{-1}$ and $317 \mathrm{~cm}^{-1}$ from the peak $2^{\prime}$, so it seems that the $300 \mathrm{~cm}^{-1}$ progression stops at peak 2 . It is true that the signal-to-noise ratio of this band is not very good and the spacings between either $\mathrm{f}_{3^{\prime}}$ or $a_{3}$ and peak $2^{\prime}$ fall almost within the experimental uncertainty of the $300 \mathrm{~cm}^{-1}$ progression. However, the $a_{3^{\prime}}$ and $f_{3^{\prime}}$ peaks are well resolved from each other, while the peaks $2^{\prime}, 1^{\prime}$ and $\sigma^{\prime}$ are poorly resolved from the adjacent transitions. Thus, the pattern of transitions : , the fourth group is clearly different from the lower energy groups of peaks.

Since the band $B^{\prime}$ does not span the energetic extent of band $B$ in the PES, there must be at least one "dark" state lying close to the ${ }^{1} \mathrm{~B}_{3 \mathrm{y}}$ state. By this, we mean a $\mathrm{Si}_{4}$ electronic state which is not accessible by s-wave detachment and therefore is seen in the PES but not the ZEKE spectrum; this includes all three of the other eiectronic states predicted to occur in the vicinity of band $B$. Hence, a possible explanation for the vibrational irregularity of band $B^{\prime}$ is that the higher vibrational levels of the ${ }^{9} B_{3 u}$ state are vibronically coupled to one of these "dark" states.

As examples of possible vibronic coupling schemes, any totally symmetric vibrational level of the ${ }^{1} \mathrm{~B}_{3 u}$ state could be coupled with odd vibrational levels in a $\mathrm{b}_{1 \mathrm{u}}$ mode of the ${ }^{1} \mathrm{~B}_{2 \mathrm{~g}}$ state, odd $b_{2 u}$ levels of the ${ }^{3} B_{1 g}$ state, or odd $b_{u}$ levels of the ${ }^{3} B_{g}$ state. Given that the three candidate states for vibronic coupling are all predicted to lie within $0.3 \mathrm{eV}$ of where we observe the ${ }^{1} \mathrm{~B}_{3 u}$ state, one might expect the dominant coupling to be with the ${ }^{1} \mathrm{~B}_{2 g}$ state, as this is the only nearby state with the same spin multiplicity.

The peak pattern for the fourth group is what one might expect under the following circumstances. Suppose $a v_{4}=$ odd level $\left[b_{1 u}\right.$ vibrational symmetry-see Figure (7.7)] of the ${ }^{1} B_{2 g}$ state were accidentally degenerate with the $v_{2}=3$ level of the ${ }^{1} B_{3\lrcorner}$ state, at the energy where peak $3^{\prime}$ would normally occur. The two vibronically coupled levels will mix and repel one 
another, and a $38 \mathrm{~cm}^{-1}$ splitting via this interaction would yield peaks $a_{3}$, and $f_{3^{\prime}}$. Peak $a_{3}$, then consists of two overlapped transitions-one due to the higher energy transition resulting from the vibronic ccupling interaction, and the other from the transition analogous to peaks $a_{0} \cdot a_{2}$ (see below). Clearly, such a mechanism must be regarded as speculative, but it is certainly not unreasonable given the large number of nearby electronic states.

The $4^{\prime}$ group is most likely affected in a similar manner, but due to the poor $S / N$, it is difficult to get a precise idea of what could be happenir"s with it. The chance of vibronic coupling only increases at higher energies due to presence of more combination bands with the appropriate vibronic character, so we would only expect it to be increasingly difficult to analyze these higher energy peaks.

Thers remain several other issues regarding this band. It has been mentioned that the profiles of the first three groups of peaks in the $B^{\prime}$ band are qualitatively similar to those in the $A^{\prime}$ band, but not exactly. For example, the spacing between peaks $a_{n^{\prime}}$ and $n^{\prime}$ in band $B^{\prime}$ is slightly smaller than between peaks $a_{n}$ and $\eta$ in band $A^{\prime}$. If the $a_{n}$, peaks are due to sequence bands in the $v_{G}$ mode, a difference between the neutral singlet and triplet $v_{6}$ frequencies of about 5 to 10 $\mathrm{cm}^{-1}$ (the triplet frequency being higher) would account for this. Also, the origin of this band has not been definitively identified, although we assign it to peak $0^{\prime}$, the central peak of the lowest energy group identifled with band B'. However, the lower signal to noise associated with band $B^{\prime}$ may cause the actual origin to be obscured. The excitation energy of the ${ }^{1} B_{3 u}$ state assuming peak $\sigma^{\prime}$ to be the origir. is $1.37=0.010 \mathrm{eV}$ [sce Table (7.4)]. While we cannot determine the origins of the ${ }^{1} \mathrm{~B}_{2 \mathrm{~g}},{ }^{3} \mathrm{~B}_{\mathrm{g}}$ or ${ }^{3} \mathrm{~B}_{1 \mathrm{~g}}$ states, our vibronic coupling hypothesis requires at least one of them to lie quite close to the ${ }^{1} \mathrm{~B}_{3 u}$ state. 


\section{Band $\mathrm{C}^{\prime}$}

While not much information can be extracted from the broad features comprising band $C^{\prime}$, a few words on its assignment are warranted. This band, which was assigned to the ${ }^{3} B_{1 u}+$ $\mathrm{e}^{-} \leftarrow{ }^{2} \mathrm{~B}_{2 \mathrm{~g}}$ transition, lies approximately $1.2 \mathrm{eV}$ above band $\mathrm{A}^{\prime}$. This raises the possibility that band $C^{\prime}$ is due to the transition to the ${ }^{1} B_{3 u}$ state (the singlet-triplet splitting for $B_{3 u}$ was calculated to be $1.04 \mathrm{eV}$ and that band $\mathrm{B}^{\prime}$ is the ${ }^{3} \mathrm{~B}_{1 \mathrm{u}}+\mathrm{e}^{-} \leftarrow{ }^{2} \mathrm{~B}_{2 g}$ transition. However, this possibility is unlikely for the following reason. From the $a b$ initio geometries, the $v_{1}$ mode should be active in transitions to the ${ }^{3} \mathrm{~B}_{1 u}$ state. The $v_{1}$ frequencies of all of the excited $\mathrm{D}_{2 n} \mathrm{Si}_{4}$ states are around $400 \mathrm{~cm}^{-1}$ or greater. The $B^{\prime}$ progression is approximately $300 \mathrm{~cm}^{-1}$, while the peak spacing in band $C^{\prime}$ is roughly $430 \mathrm{~cm}^{-1}$, consistent with a $v_{1}$ progression. We therefore believe that the assignment of band $C^{\prime}$ made by Rohlfing and Raghavachar is correct. 4

\section{v. Conclusion}

The results presented here represent a far more detailed experimental probe of the vibrational and electronic structure of silicon tetramer than our frevious photoelectron spectroscopy study. We have obtained several vibrational frequencies for the anion and various electronic states of the neutral, particularly the ${ }^{3} \mathrm{~B}_{3 \mathrm{u}}$ excited state, as well as excitation energies for several electronic states.

Our results also emphasize the interaction between theory and and experiment in understanding the spectroscopy of clusters. The role of ab initio calculations in interpreting these spectra cannot be overemphasized. In the absence of experimental force constants for $\mathrm{Si}_{4}, a b$ initio calculations are needed to perform any reasonable assignment of the observed vibrational progressions. Moreover, given the complex electronic structure of a species such as 
$\mathrm{Si}_{4}$, the assignment of electronic bands to specific ntutral $\leftarrow$ anion transitions also requires some ab initio input.

Our experimental results provide a detailed comparison vith the calculations. We find from band $A^{\prime}$ that the excitation energy for the $\mathrm{Si}_{4}{ }^{3} \mathrm{~B}_{3 u}$ state is in excellent agreement, as are the vibrational frequencies for the $v_{2}$ mode in the anion and this state of the neutral. The sequence band structure in band $A^{\prime}$ is consistent with the $a b$ initio values for the $v_{3}$ mode in the anion and neutral, but not with the $a b$ initio values for the $v_{5}$ and $v_{6}$ modes. Our results also show that band $\mathrm{B}$ in the $\mathrm{Si}_{4}^{-}$photoelectron spectrum is due to transitions to overlapping electronic states, as suggested in the calculations, but the ZEKE spectrum implies that the lowest energy of these is the $\mathrm{Si}_{4}{ }^{1} \mathrm{~B}_{3 u}$ state, whereas the calculations predict this to be about 0.5 eV higher than where we observe it. Finally, on the basis of the observed vibrational progression, we find the ab initio assignment of band $\mathrm{C}$ to the transition to the $\mathrm{Si}_{4}{ }^{3} \mathrm{~B}_{14}$ state to be reasonable.

We feel that these comparisons between experiment and theory will become even more essential as we begin to study the larger Si clusters via threshold photodetachment spectroscopy. Many of these are predicted to exhibit several low-iying structures with very different geometries. A comparison of experimental and theoretical vibrational frequencies and electronic excitation energies will facilitate soiting out these possible cluster structures.

\section{Acknowledgements}

This research is supported by the National Science Foundation under Grant No. DMR9201159. We thank C. M. Rohifing for several stimulating discussions. 
'For example, see P. W. Merrill, Publ. Astron. Soc. Pac. 38, 175 (1926); R. F. Sanford, Astrophys. J. 111, 262 (1950); B. Kleman, Astrophys. J. 123, 162 (1950) and references therein.

2P. Ho and W. G. Breiland, App. Phys. Lett. 44, 51 (1984).

3K. Raghavachari and C. M. Rohifing, J. Chem. Phys. 94, 3670 (1991); C. M. Rohifing and K. Raghavachari, Chem. Phys. Lett. 167, 559 (1990); K. Raghavachari, Z. Phys. D 12, 61 (1989); K. Raghavachari, J. Chem. Phys. 84, 5672 (1986); K. Raghavachari and V. Logovinsky, Phys. Rev. Lett. 55, 26 (1985); K. Raghavachari, J. Chem Phys. 83, 3520 (1985).

${ }^{4} \mathrm{C}$. M. Rohlfing and K. Raghavachari, J. Chem. Phys. 96, 2114 (1992).

${ }^{5}$. Fournier, S. B. Sinnott, and A. DePristo, J. Chem. Phys. 97, 4149 (1992); S. D. Li, R. L. Johnston, and J. N. Murrell, J. Chem. Soc.- Faraday Trans. 88, 1229 (1992);D. G. Dai and K. Balasubramanian, J. Chem. Phys. 96, 3279 (1992); L. Adamowicz, Chem. Phys. Lett. 188, 131 (1992); Chem. Phys. Lett. 185, 244 (1991); S. Katircioglu and S. Erkoc, Chem. Phys. Lett, 184, 118 (1991); K. Balasubramanian, Chem. Phys. Lett. 135, 283 (1987); Chem. Phys. Lett. 125, 400 (1986); G. Pacchioni and J. Koutecky, J. Chem. Phys. 84, 3301 (1986); J. R. Sabin, J. Oddershede, G. H. F. Diercksen, and N. E. Gruner, J. Chem. Phys. 84, 354 (1986); Z. Slanina, Chem. Phys. Lett. 131, 420 (1986); R. S. Grev and H. F. Schaefer, Chem. Phys. Lett. 119, 111 (1985); R. O. Jones, Phys. Rev. A 32, 2589 (1985).

6A. E. Douglas, Can. J. Phys. 33, 801 (1955); R. D. Verma and P. A. Warsop, Can. J. Phys. 41, 152 (1963); I. Dubois and H. Leclerq, Can. J. Phys. 49, 3053 (1971); S. P. Davis and J. W. Brault, J. Opt. Soc. Am. B 4, 20 (1987).

${ }^{7}$ For example, see J. R. Heath, Y. Liu, S. C. O'Brien, Q.-L. Zhang, R. F. Curl, K. Tittel, and R. E. Smalley, J. Chem. Phys. 83, 5520 (1985); Y. Liu, O.-L. Zhang, F. K. Tittel, R. F. Curl, and R. E. Smalley, J. Chem. Phys. 85, 7434 (1986); L. A. Bloomfield, R. R. Freeman, and W. L. Brown, Phys. Rev. Lett. 54, 2246 (1985). 
BJ. R. Heath, Y. Liu, S. C. O'Brien, Q.-L. Zhang, R. F. Curl, F. K. Tittel, and R. E. Smalley, J. Chem. Phys, 83, 5520 (1985).

9M. L. Mandich, V. E. Bondybey, and W. D. Reents, Jr., J. Chem. Phys. 86, 4245 (1987).

10W. Weltner, Jr. and D. McLeod, Jr., J. Chem. Phys. 41, 235 (1964).

11T. P. Martin and H. Schaber, Z. Physik B 35, 61 (1979).

${ }^{12}$ C. B. Winstead, K. X. He, T. Hammond, and J. L. Gole, Chem. Phys. Lett. 181, 222 (1991).

${ }^{13}$ C. Jin, K. J. Taylor, J. Conceicao, and R. E. Smalley, Chem. Phys. Lett. 175, 17 (1990).

140. Cheshnovsky, S. H. Yang, C. L. Pettiette, M. J. Craycraft, Y. Liu, and R. E. Smalley, Chem. Phys. Lett. 138, 119 (1987).

15M. R. Nimlos, L. B. Harding, and G. B. Ellison, J. Chem. Phys. 87, 5116 (1987).

16T. N. Kitsopoulos, C. J. Chick, Y. Zhao, and D. M. Neumark, J. Chem. Phys. 95, 5479 (1991); C. C. Arnold, T. N. Kitsopoulos, and D. M. Neumark, J. Chem. Phys. (in press).

17T. N. Kitsopoulos, C. J. Chick, A. Weaver, and D. M. Neumark, J. Chem. Phys. 93, 6108 (1990).

${ }^{18}$ For example, see K. J. Taylor, C. L. Pettiette, O. Cheshnovshy, and R. E. Smalley, J. Chem. Phys. 96, 3319 (1992); J. Ho, K. M. Ervin, and W. C. Lineberger, J. Chem. Phys. 93, 6987 (1990); J. G. Eaton, H. W. Sarkas, S. T. Amold, K. M. McHugh, and K. H. Bowen, Chem. Phys. Lett. 193, 141 (1992); G. Gantefor, M. Gausa, K. H. Mejwesbroer, H. O. Lutz, Faraday Disc. Chem. Soc. 86, 197 (1988); Z. Phys. D 9, 253 (1988); S. M. Casey, P. W. Villalta. A. A. Bengali, G. L. Cheny, and D. G. Leopold, J. Am. Chem. Soc. 113, 6688 (1991). 
19L. S. Wang, J. Conceicao, C. M. Jin, and R. E. Snalley, Chem. Phys. Lett. 182, 5 (1991); S. Yang, K. J. Taylor, M. J. Craycraft, J. Conceicao, C. L. Pettiette, O. Cheshnovsky, and R. E. Smalley, Chem. Phys. Lett. 144, 431 (1988); C. C. Amolr', Y. Zhao, T. N. Kitsopoulos, and D. M. Neumark, J. Chem. Phys. 97, 6121 (1992); D. W. Amold, S. E. Bradforth, T. N. Kitsopoulos, and D. M. Neumark, J. Chem. Phys. 95, 8753 (1991); T. N. Kitsopoulos, C. J. Chick, Y. Zhao, and D. M. Neumark, i. Chem. Phys. 95, 5479 (1991).

20K. Müller-Dethlefs, M. Sander, and E. W. Schlag, Z. Naturiorsch 39a, 1089 (1984); Chern. Phys. Lett. 12, 291 (1984); K. Müller-Dethlefs and E. W. Schlag, Ann. Rev. Phys. Chem. 42, 109 (1991).

${ }^{21}$ E. P. Wigner, Phys. Rev. 73, 1002 (1948).

22K. J. Reed, A. H. Zimmerman, H. C. Andersen, and J. I. Brauman, J. Chem. Phys. 64, 1368 (1976).

${ }^{23}$ T. N. Kitsopoulos, I. M. Waller, J. G. Loeser, and D. M. Neumark, Chem. Phys. Lett. 159, 300 (1989); T. N. Kitsopoulos, C. J. Chick, Y. Zhao, and D. M. Neumark, J. Chem. Phys. 95, 1441 (1991).

24T. G. Dietz, M. A. Duncan, D. E. Powers, and R. E. Smalley, J. Chem. Phys. 74, 6511 (1981).

25D. Proch and T. Trickl, Rev. Sci. Instrum. 60, 713 (1989).

${ }^{26}$ C. C. Amold, Y. Zhao, T. N. Kitsopoulos, and D. M. Neumark, J. Chem. Ptiys. 97, 6121 (1992). 
Table (7.1) Positions, spacings and tentative assignments for the main peaks in the $A^{\prime}$ band of $\mathrm{Si}_{4}^{-}$

\begin{tabular}{|c|c|c|c|}
\hline Peak & $\begin{array}{l}\text { Position } \\
\text { (nm) }\end{array}$ & $\begin{array}{l}\text { Spacing } \\
\left(\mathrm{cm}^{-1}\right)^{a}\end{array}$ & $\begin{array}{l}\text { Tentative } \\
\text { Assignment }^{b}\end{array}$ \\
\hline 0 & $415.27(14)$ & \multirow{5}{*}{$\begin{array}{l}314 \\
311 \\
308\end{array}$} & Origin \\
\hline 1 & $409.93(13)$ & & 21 \\
\hline 2 & $404.77(13)$ & & 2 \\
\hline 3 & $399.78(13)$ & & $23_{0}^{3}$ \\
\hline 4 & $394.84(12)$ & & 24 \\
\hline 5 & $390.06(12)$ & \multirow{3}{*}{$\begin{array}{l}310 \\
311 \\
308\end{array}$} & 25 \\
\hline 6 & $385.39(12)$ & & $2_{0}^{6}$ \\
\hline 7 & $380.87(12)$ & & 27 \\
\hline 8 & $376.46\langle 11\rangle$ & \multirow[t]{2}{*}{$\begin{array}{l}308 \\
308\end{array}$} & $\overline{2_{0}^{8}}$ \\
\hline 9 & $372.15(11)$ & & $2_{0}^{9}$ \\
\hline 10 & $367.98(11)$ & \multirow{2}{*}{$\begin{array}{l}305 \\
310\end{array}$} & $2_{0}^{10}$ \\
\hline 11 & $363.83(11)$ & & $2_{0}^{11}$ \\
\hline 12 & $359.83(10)$ & \multirow{2}{*}{$\begin{array}{l}306 \\
303\end{array}$} & 212 \\
\hline 13 & $355.95(10)$ & & $20_{0}^{13}$ \\
\hline
\end{tabular}

a Error for each spacing is $\pm 11 \mathrm{~cm}^{-1}$

${ }^{b}$ Based on peak 0 being the origin 
Table (7.2) Positions, relative spacings, and tentative assignment of sequence bands found around peaks 3 and 4 in the $\mathrm{A}^{\prime}$ band of $\mathrm{Si}_{4}{ }^{-}$.

\begin{tabular}{|l|l|l|l|}
\hline Peak & $\begin{array}{l}\text { Position } \\
(n m)\end{array}$ & $\begin{array}{l}\text { Spacing from } \\
\text { main feature } \\
\left(\mathrm{cm}^{-4}\right)^{\mathrm{a}}\end{array}$ & $\begin{array}{l}\text { Tentative } \\
\text { assignment }\end{array}$ \\
\hline$g_{3}$ & $400.74(13)$ & -59 & $2_{1}^{4}$ \\
\hline$f_{3}$ & $400.13(13)$ & -21 & $2_{0}^{3} 3_{1}^{1}$ \\
\hline 3 & $399.78(13)$ & 0 & $2_{0}^{3}$ \\
\hline$a_{3}$ & $399.40(13)$ & 24 & $2_{0}^{3} 6_{1}^{1}$ \\
\hline$b_{3}$ & $398.94(13)$ & 53 & $2_{0}^{3} 5_{1}^{1}$ \\
\hline$c_{3}$ & $308.56(13)$ & 74 & $2_{0}^{3} 5_{1}^{1} 6_{1}^{1}$ \\
\hline$d_{3}$ & $398.09(13)$ & 107 & $2_{0}^{3} 5_{2}^{2}$ \\
\hline$e_{3}$ & $397.34(13)$ & 154 & $2_{0}^{3} 5_{3}^{3}$ \\
\hline & & & \\
\hline$g_{4}$ & $395.56(12)$ & -46 & $2_{1}^{5}$ \\
\hline$f_{4}$ & $395.19(12)$ & -22 & $2_{0}^{4} 31$ \\
\hline 4 & $394.84(12)$ & 0 & $2_{0}^{4}$ \\
\hline$a_{4}$ & $394.39(12)$ & 29 & $2_{0}^{4} 6_{1}^{1}$ \\
\hline$b_{4}$ & $393.99(12)$ & 55 & $2_{0}^{4} 5_{1}^{1}$ \\
\hline$c_{4}$ & $393.64(12)$ & 77 & $2_{0}^{4} 5_{1}^{1} 6_{1}^{1}$ \\
\hline$d_{4}$ & $393.29(12)$ & 100 & $2_{0}^{4} 5_{2}^{2}$ \\
\hline
\end{tabular}

a Error for each spacing is $\pm 11 \mathrm{~cm}^{-1}$ 
Table (7.3) Positions and relative spacings of the peaks found in the $\mathrm{B}^{\prime}$ band of $\mathrm{Si}_{4}-$.

\begin{tabular}{|c|c|c|}
\hline Peak & $\begin{array}{l}\text { Position } \\
(\mathrm{nm})\end{array}$ & $\begin{array}{l}\text { Relative } \\
\text { spacing }\left(\mathrm{cm}^{-1}\right)^{a}\end{array}$ \\
\hline $0^{\prime}$ & $349.90(12)$ & 0 \\
\hline$a_{0^{\prime}}$ & $349.70(12)$ & 16 \\
\hline$b_{0}$ & $349.35(12)$ & 45 \\
\hline$g_{1}$ & $347.0(12)$ & 239 \\
\hline $1^{\prime}$ & $346.20(12)$ & 305 \\
\hline$a_{1}$ & $345.86(12)$ & 334 \\
\hline$b_{1}$ & $345.40(14)$ & 372 \\
\hline$g_{2^{\prime}}$ & $343.61(12)$ & 529 \\
\hline$\overline{z^{\prime}}$ & $342,69(12)$ & 602 \\
\hline$a_{2}$ & $342.41(12)$ & 625 \\
\hline$b_{2}$ & $342.13(14)$ & 649 \\
\hline$g_{3^{\prime}}$ & $340.22(12)$ & 814 \\
\hline$f_{3}$ & $339.45(8)$ & 880 \\
\hline$\ldots$ & $\cdots$ & $\cdots$ \\
\hline$a_{g^{\prime}}$ & $339.00(8)$ & 918 \\
\hline$b_{3^{\prime}}$ & $338.60(11)$ & 954 \\
\hline$g_{4^{\prime}}$ & $337.21(17)$ & 1076 \\
\hline$f^{\prime}$ & $336.26(11)$ & 1160 \\
\hline $4^{\prime} ?$ & $336.96(11)$ & 1186 \\
\hline$a_{4^{\prime}}$ & $335.61(15)$ & 1217 \\
\hline $5^{\prime}$ & $332.71(11)$ & 1477 \\
\hline
\end{tabular}

aError for spacing is $\pm 14 \mathrm{~cm}^{-1}$ 
Table (7.4) Excitation energies for several of the low-lying states of $\mathrm{Si}_{4}$.

\begin{tabular}{|l|l|}
\hline State of $\mathrm{Si}_{4}$ & $\begin{array}{l}\mathrm{T}_{\theta} \\
(\mathrm{eV})\end{array}$ \\
\hline${ }^{3} \mathrm{~B}_{1 U}$ & $2.01 \pm 0.02$ \\
\hline${ }^{1} \mathrm{~B}_{3 u}$ & $1.37 \pm 0.01$ \\
\hline${ }^{3} \mathrm{~B}_{3 u}$ & $0.815 \pm 0.010$ \\
\hline${ }^{1} \mathrm{~A}_{9}$ & $\begin{array}{l}0 \\
\left(E . A_{0}=2.17 \pm 0.01\right)\end{array}$ \\
\hline
\end{tabular}


Table (7.5) $\mathrm{Si}_{4}$ neutral and anion frequencies along with anion vibrational temperature assumed for the spectral simulation shown in Figure (7.7). The alternative interpretation of the spectrum for $v_{5}$ and $v_{6}$ is shown in parenthesis. Also included are calculated anion frequencies.

\begin{tabular}{|l|l|l|l|l|}
\hline Mode & $\begin{array}{l}\text { Neutral } \\
\text { frequency } \\
\left(\mathrm{cm}^{-1}\right)\end{array}$ & $\begin{array}{l}\text { Anion } \\
\text { frequency } \\
\left(\mathrm{cm}^{-1}\right)\end{array}$ & $\begin{array}{l}\text { Temperature } \\
(\mathrm{K})\end{array}$ & $\begin{array}{l}\text { Calc'd } \\
\text { Anion } \\
\text { freq. }\left(\mathrm{cm}^{-1}\right)\end{array}$ \\
\hline$v_{2}$ & $312^{\mathrm{a}}$ & 365 & 200 & 393 \\
\hline$v_{3}$ & 367 & 392 & 270 & 390 \\
\hline$v_{5}$ & 177 & $125(150)$ & $170(380)$ & 295 \\
\hline$v_{6}$ & 159 & $132(107)$ & $290(120)$ & 165 \\
\hline
\end{tabular}

${ }^{a}$ Calculated neutral frequency for $v_{2}$ is $306 \mathrm{~cm}^{-1}$.

Bef. (4) 
Figure (7.1) Photoelectron spectra of $\mathrm{Si}_{4}{ }^{-}$[Ref. (17)] obtained using $3.493 \mathrm{eV}$ photon energy (top panel) and $4.66 \mathrm{eV}$ photon energy (bottom panel) with the assignments of Rohifing and Raghavachari [Ref. (4)].
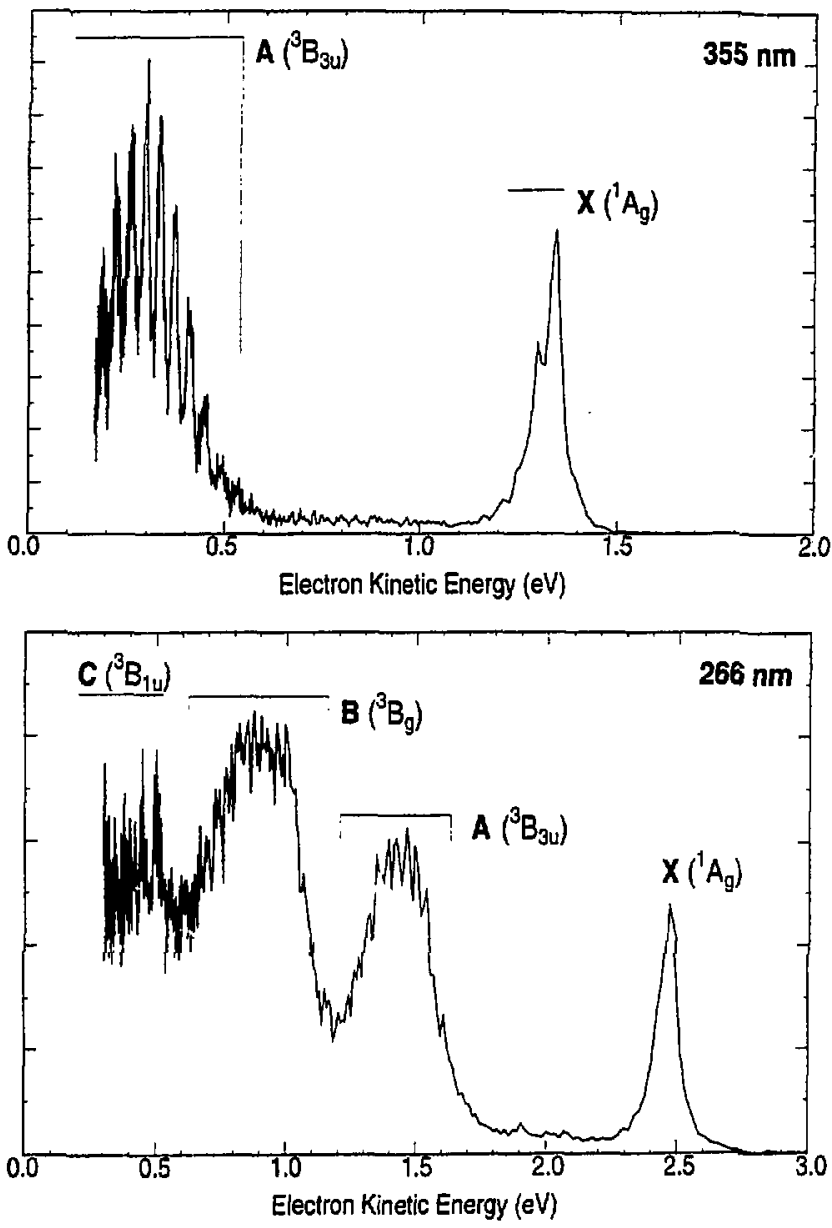
Figure (7.2) Diagram of the tunable fhotodetachment apparatus: $(x)$ interaction region; (1) detachment laser windows; (2) extraction plates and appertures; (3) magnetic shielding; (4) electron einzel lens; (5) ion detector (6) slectron detector (above the plane).

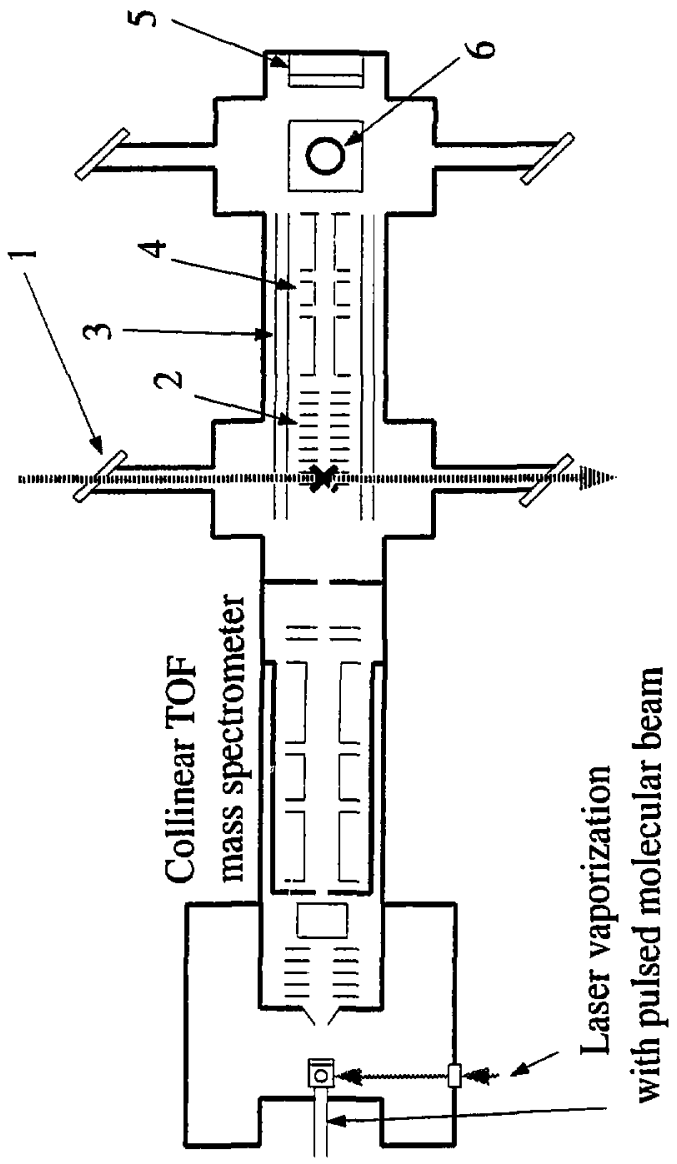


Figure (T.3) The ZEKE spectrum of $\mathrm{Si}_{4}{ }^{-}$(solid lines) is superimposed onto the energy scale of the PES of $\mathrm{Si}_{4}{ }^{-}$obtained using $4.66 \mathrm{eV}$ photon energy (dotted tine).

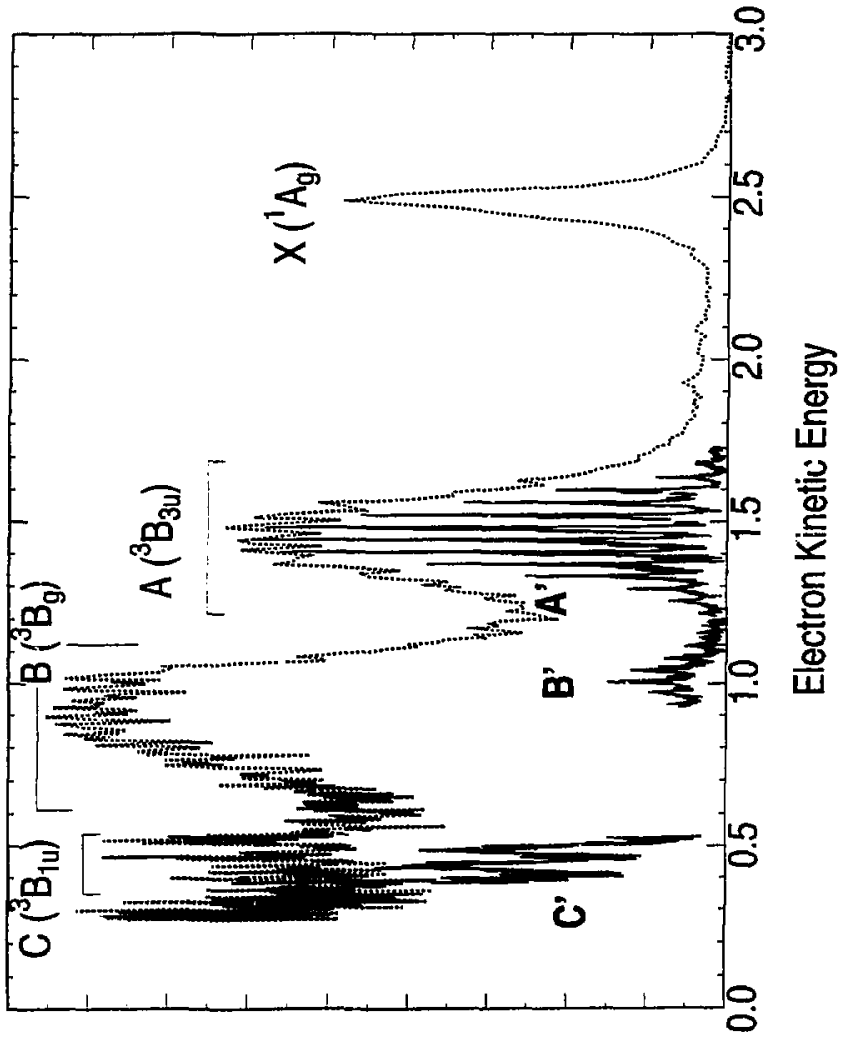




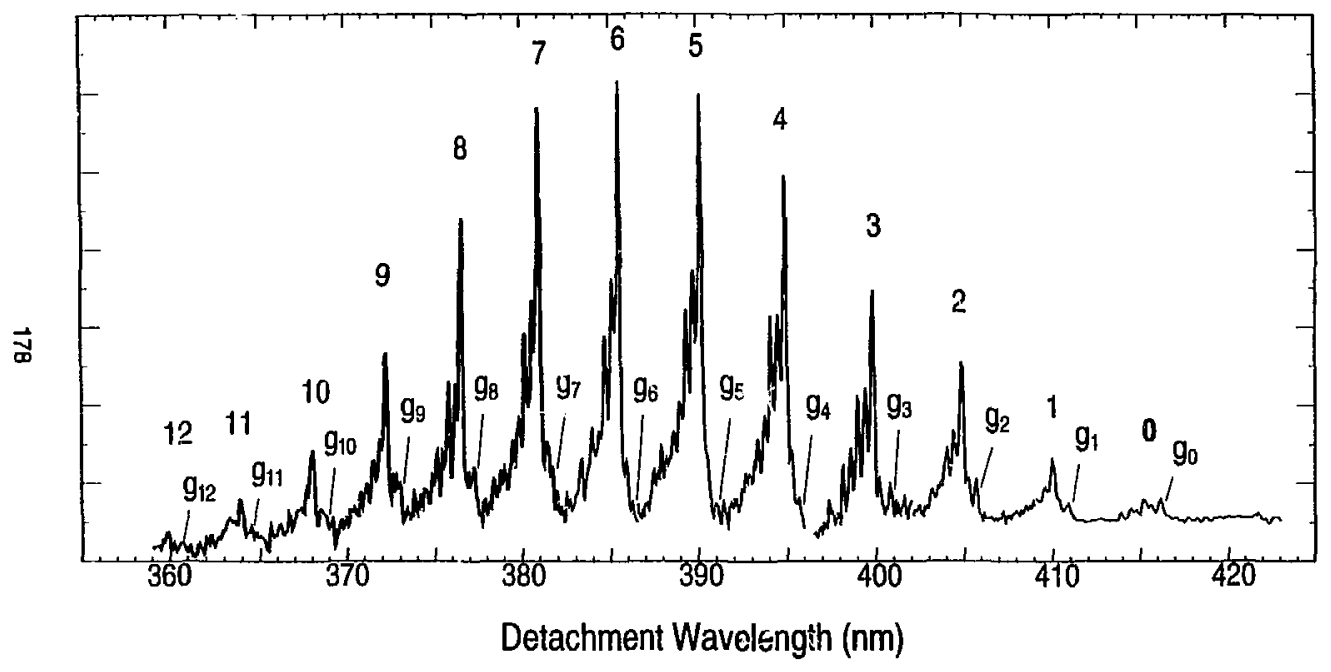

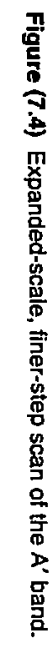


Figure (7.5) Expanded-scale, finer-step scan of peaks 3 and 4 of the $A^{\prime}$ band.

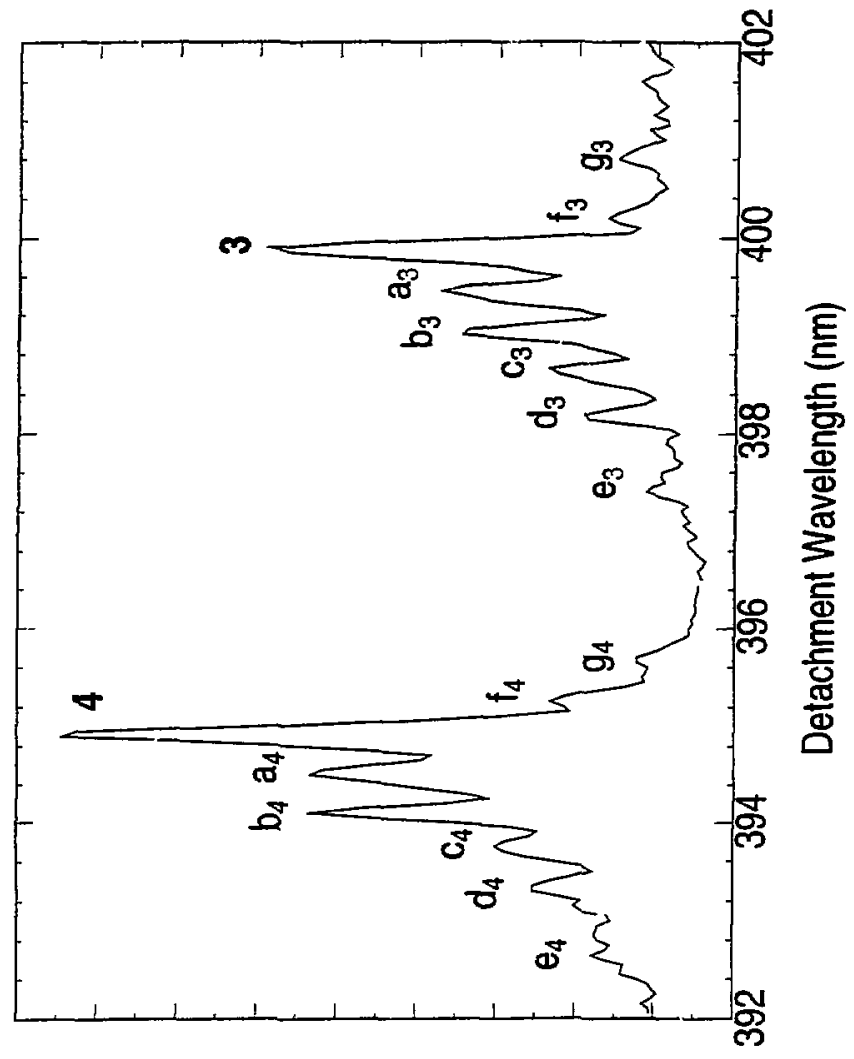




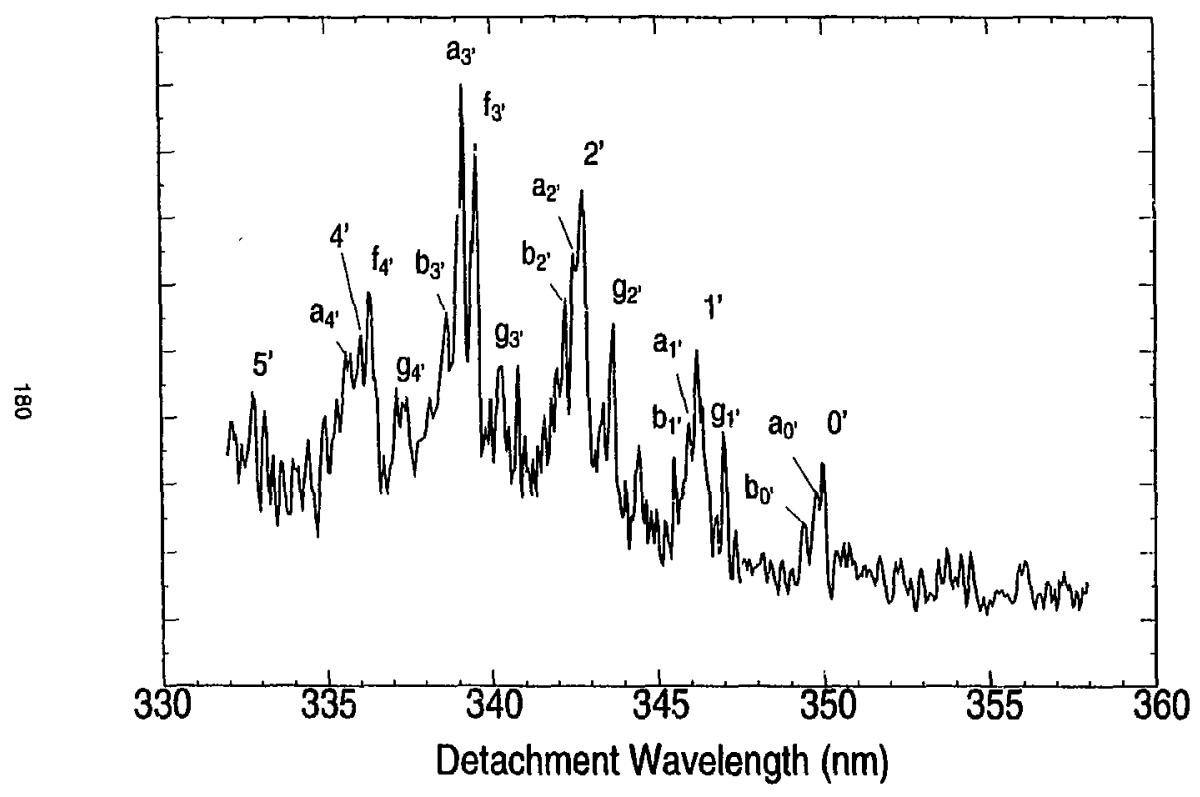

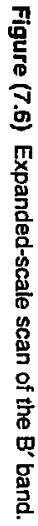


Figure (7.7) Calculated geometries of the ground ${ }^{2} \mathrm{~B}_{2 g}$ anion state and the neutral ${ }^{3} \mathrm{~B}_{3 u}$ state [Ref. (4)], the orbital from which an electron is removed in the transition between the two states, and the normal coordinates of the ${ }^{3} \mathrm{~B}_{3 u}$ state of $\mathrm{Si}_{4}$.
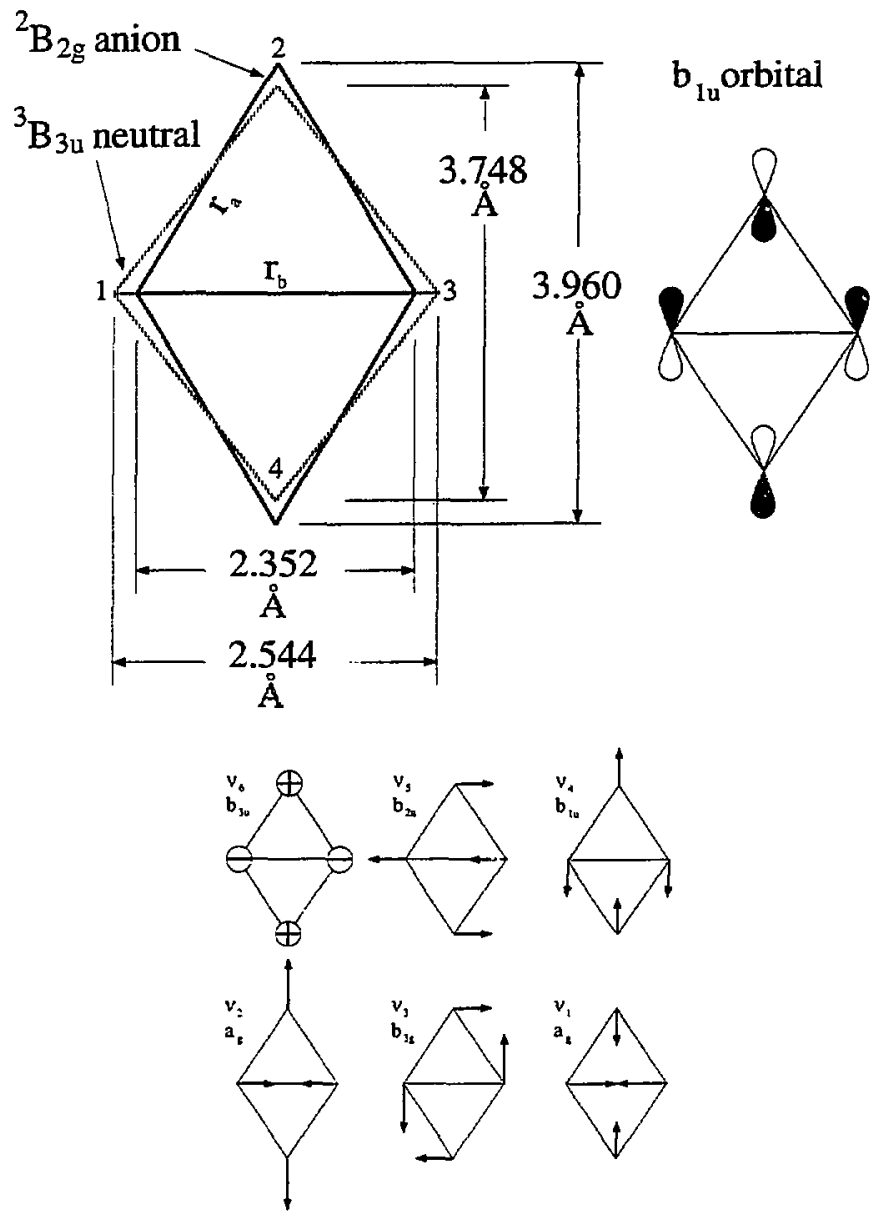
Figure (7.8) One-dimensional potential energy curves for the anion and neutral $v_{2}$ modes. The anion potential is displaced by the amount determined in the simulation of band $A^{\prime}$.

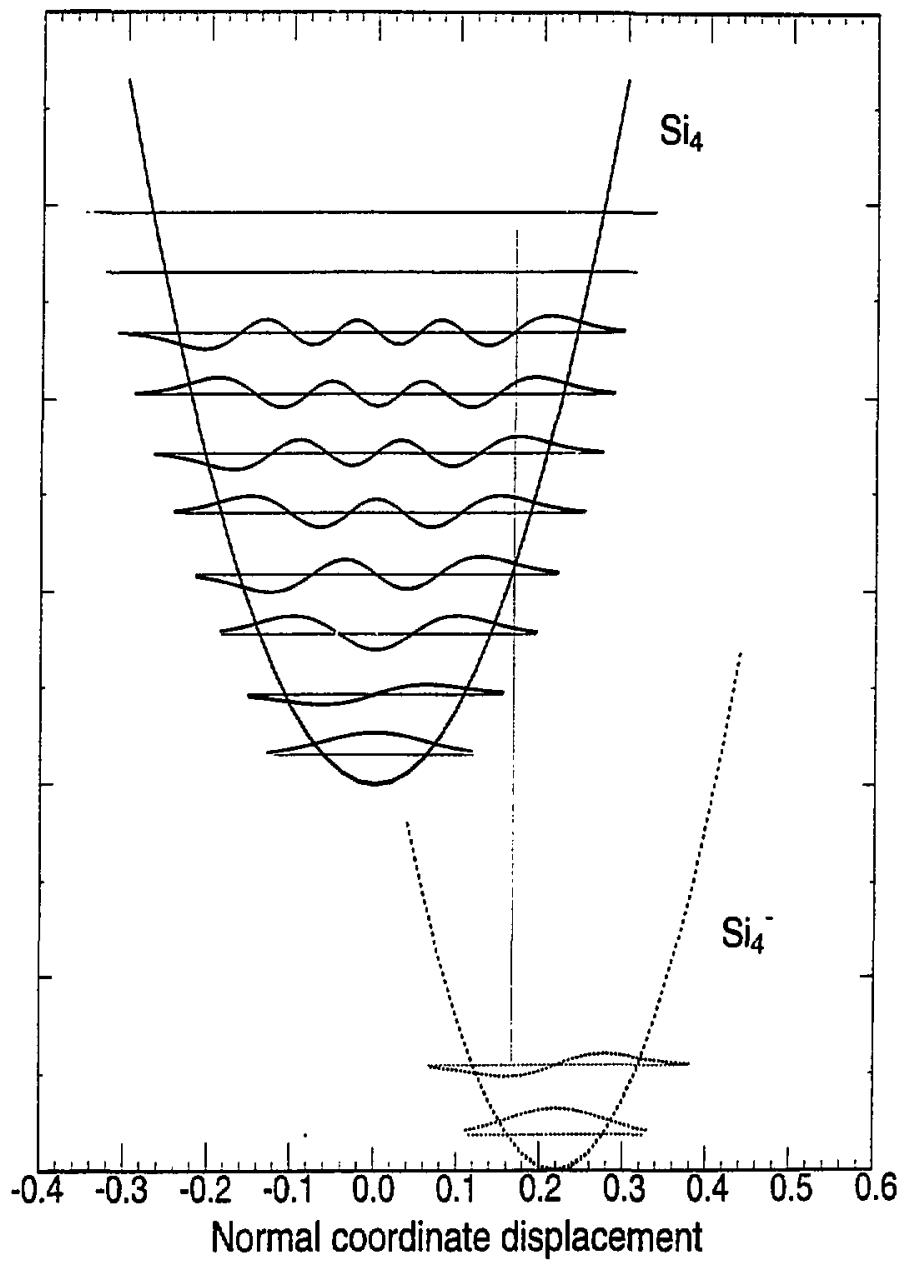


Figure (7.9) Four mode Franck-Condon simulation (solid line) of the $A^{\prime}$ band (circles). The inset shows an expanded scale view of the simulation. Those peaks labelled explicitly by $n$ or $g_{n}$ are due to excitations in the $y_{2}$ mode of the anion and/or neutral.

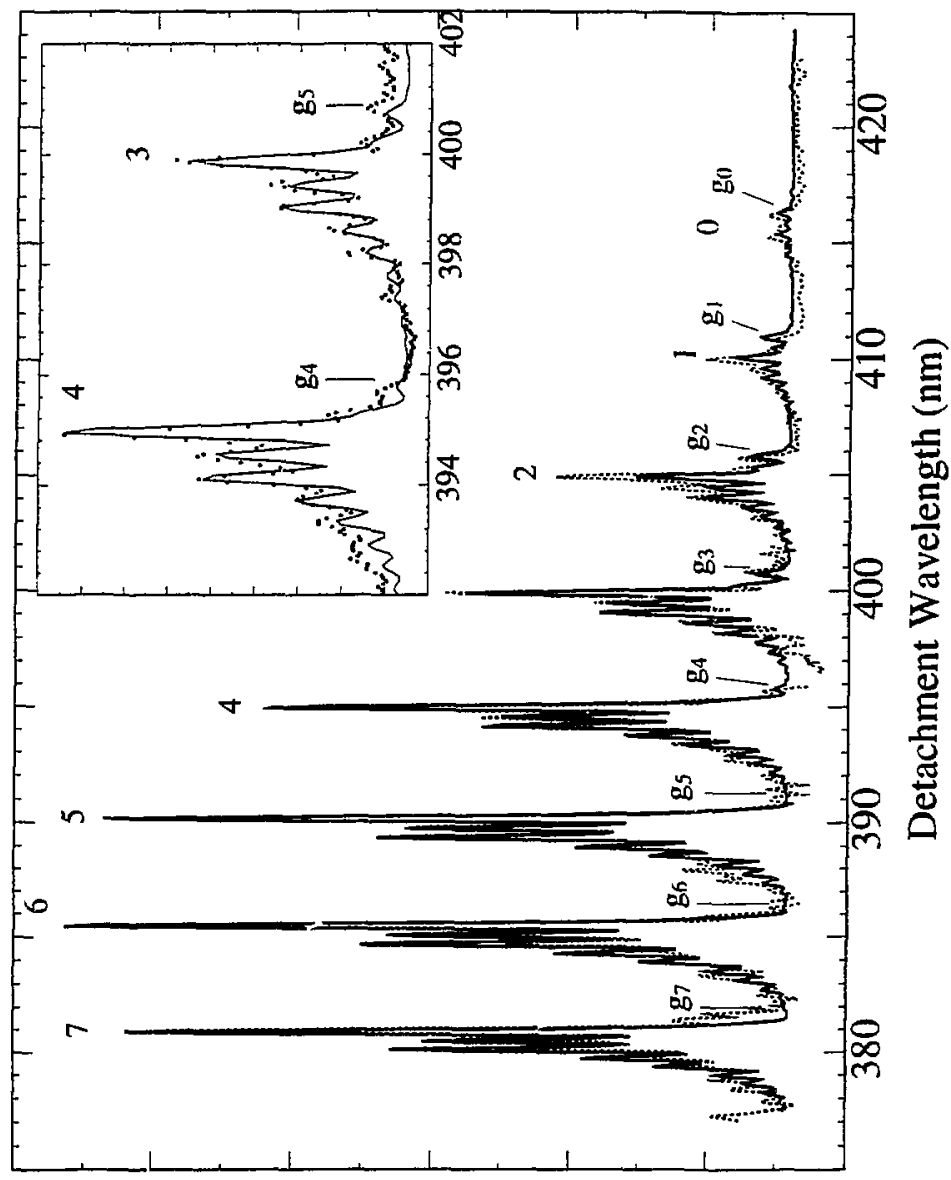




\section{Chapter 8. Study of the low-lying states of $\mathrm{Ge}_{2}$ and $\mathrm{Ge}_{2}^{-}$using negative ion ZEKE spectroscopy}

\section{Abstract}

The projicities of the low-lying states of $\mathrm{Ge}_{2}$ and $\mathrm{Ge}_{2}{ }^{-}$are probed using negative ion zero electron kinetic energy (ZEKE) spectroscopy. The ZEKE spectrum of $\mathrm{Ge}_{2}^{-}$yields and electron affinity of $2.035 \pm 0.001 \mathrm{eV}$ for $\mathrm{Ge}_{2}$, as well as term energies and vibrational frequencies for the low-lying states of $\mathrm{Ge}_{2}{ }^{+}$and $\mathrm{Ge}_{2}$. The anion has a ${ }^{2} \Pi_{\mathrm{u}}(3 / 2)$ ground state ( $\omega$ $\left.=309 \pm 5 \mathrm{~cm}^{-1}\right)$ and a $2 \Sigma_{g}+\left(\omega=326 \pm 10 \mathrm{~cm}^{-1}\right)$ at $T_{e} 279 \pm 10 \mathrm{~cm}^{-1}$. The neutral has a ${ }^{2} \Sigma_{g}+$ ground state $\left(\omega=286 \pm 5 \mathrm{~cm}^{-1}\right.$ ) exhibiting a $114 \pm 5 \mathrm{~cm}^{-1}$ zero field splitting between the $\mathrm{XO}_{\mathrm{g}}{ }^{*}$ and $1_{g}$ components. The ${ }^{3} \Pi_{u}$ tirst excited state $\Pi_{e}=694 \mathrm{~cm}^{-1}, \omega=308.0 \mathrm{~cm}^{-1}$ from $\mathrm{Li}$, Van Zee, and Weltner, J. Chem. Phys. 100, 7079 (1994)] has a spin-coupling constant, $A$, of approximately $484 \mathrm{~cm}^{-1}$, with the $2_{u}, 1_{u}$ and $\mathrm{O}_{u}^{ \pm}$components not symmetrically spaced. The manifold of low-lying singlet states was also probed, yielding $T_{\theta}=3300 \pm 5 \mathrm{~cm}^{-1}$ for the ${ }^{1} \Delta_{g}$ state $\left(\omega=276 \pm 3 \mathrm{~cm}^{-1}\right), T_{e}=4941 \pm 5 \mathrm{~cm}^{-1}$ for the ${ }^{1} \Sigma_{g}{ }^{+}$state $\left(\omega=204 \pm 5 \mathrm{~cm}^{-1}\right)$ and $T_{e}=4943 \pm 10$ $\mathrm{cm}^{-1}$ for the ${ }^{1} \Pi_{\mathrm{u}}$ state $\left(\omega=303 \pm 5 \mathrm{~cm}^{-1}\right)$. 


\section{Introduction}

The ongoing effort to characterize the geometries and electronic structures of small elemental semiconductor clusters has been fueled by theoretical studies for at least ten years. However, experiments on these elusive species have proven extremely difficult, primarily due to the lack of mass-specificity of most cluster sources. For instance, only recently has a fairly complete understanding of the electronic structure of $\mathrm{Si}_{2}$ been experimentally obtained using negative ion photodetachment techniques, ${ }^{1}$ in contrast to the 14 years of partially successful $a b$ initio study, ${ }^{2}$ which is surprising in light of it's believed role in CVD processes. ${ }^{3}$ The presence of many low-lying states inaccessible from the neutral ground state further contributed to the difficulty in completely characterizing the electronic structure of the smallest silicon cluster. To date, most spectroscopic information on small metal, ${ }^{4}$ carbon, ${ }^{5}$ and semiconductor clusters ${ }^{6,7,8}$ has been accnued from mass-selective experiments, such as negative ion photodetachment.

As an extension of our previous work on $\mathrm{Si}_{2}{ }^{*}$, this paper presents the zero electron kinetic energy, or ZEKE spectnum of $\mathrm{Ge}_{2}{ }^{*}$ which, when compared with the previously obtained anion photoelectron spectrum (PES) $^{9}$ and matrix studies pertormed by Li, Van Zee, and Weltner, ${ }^{10}$ gives an accurate map of the five lowest-lying states of $\mathrm{Ge}_{2}$ and the two low-lying states of $\mathrm{Ge}_{2}$ - The germanium dimer has been predicted to be electronically very similar to silicon dimer, with a ${ }^{3} \Sigma_{g} \cdot\left(. .2 \sigma_{g}{ }^{2} 1 \pi_{u}{ }^{2}\right)$ ground state and a very low-lying ${ }^{3} \Pi_{u}\left(. .2 \sigma_{g}{ }^{1} 1 \pi_{u}{ }^{3}\right)$ first excited state having a calculated term energy $\left(T_{e}\right)$ ranging from 50 to $800 \mathrm{~cm}^{-1} .11,12,13.14$ There are also four singlet states resulting from these same orbital occupancies along with the $\left(\ldots 1 \pi_{u}{ }^{4}\right)$ occupancy predicted to lie within $1.5 \mathrm{eV}$ of the ground state, as with $\mathrm{Si}_{2}$. On the other hand, relativistic effects are expected to be more important in $\mathrm{Ge}_{2}$ than $\mathrm{Si}_{2}$. With $\mathrm{Ge}_{2}$ approaching Hund's case (c), $\Lambda$ and $\Sigma$ are no longer good quantum numbers (states having different $\Lambda$ and $\Sigma$ but the same $\Omega$ can mix) ${ }^{15.16}$ with $\Omega$ remaining good, and the spin-orbit components of the ${ }^{3} \Pi_{u}$ state may be better described as $0_{u}+0_{u}, 1_{u}$ and $2_{u}$ states $\left(2_{u}\right.$ being the lowest-lying), and the 
${ }^{3} \Sigma_{g}$ - state better described as $\mathrm{X0}_{\mathrm{g}}{ }^{+}$and $1_{g}$ states. The energy splitting between the various $\Omega$ components of $\mathrm{Ge}_{2}$ should be substantially larger than the spin-orbit splitting in $\mathrm{Si}_{2}$., for which the splitting between the ${ }^{3} \Pi_{0}$ and ${ }^{3} \Pi_{2}$ states $(2 A)$ is $145 \mathrm{~cm}^{-1}$, and the splitting between the $\mathrm{XO}_{\mathrm{g}}{ }^{+}$ and $1 \mathrm{~g}$ components of the ${ }^{3} \Sigma_{\mathrm{g}}{ }^{-}$state $(2 \lambda)$ is $2.6 \mathrm{~cm}^{-1} \cdot{ }^{10}$

The ${ }^{3} \Pi_{u}\left[1_{u}\left(v^{\prime}=0,1,2,3\right)\right] \leftarrow{ }^{3} \Sigma_{g}\left(X_{g}{ }^{+}\right)$transition was observed in Weltner's matrix absorption studies, giving a term energy of $694 \mathrm{~cm}^{-1}\left(711 \mathrm{~cm}^{-1}\right.$ between ground vibrational levels of the two states) and a $308 \mathrm{~cm}^{-1}$ vibrational frequency for the $1_{u}$ component of the ${ }^{3} \Pi_{u}$ excited state. Transitions to the other $\Omega$ components of the ${ }^{3} \Pi_{u}$ state are not allowed from the ${ }^{3} \Sigma_{g} \cdot\left(\mathrm{XO}_{\mathrm{g}}{ }^{+}\right)$state. A vibrational frequency assigned to the ${ }^{3} \mathrm{I}_{\mathrm{g}}\left(\mathrm{XO}_{\mathrm{g}}{ }^{+}\right)$state in a matrix was observed in Raman/fluorescence studies. ${ }^{17}$ To our knowledge, no spectroscopic information on the low-lying singlet states has until now been obtained.

The low-Jying singlet states, however, can be accessed using negative ion photodetachment techniques. While the more common anion PES sometimes cannot resolve close-lying or overlapping electronic transitions, negative ion ZEKE spectroscopy is particularly well suited toward small cluster study, as it combines mass-selection with good spectroscopic resolution. Anion ZEKE is most powerful when used in tandem with fixed-frequency photoelectron spectroscopy (PES) which, with its less-restrictive selection nules, is sensitive to more electronic transitions.

No $a b$ initio results are presently available for $\mathrm{Ge}_{2}^{-}$, but if it is electronically similar to $\mathrm{Si}_{2}$, it has two close-lying ${ }^{2} \Pi_{\mathrm{u}}$ and ${ }^{2} \Sigma_{\mathrm{g}}{ }^{+}$states (the ${ }^{2} \Sigma_{\mathrm{g}}{ }^{+}$state was the ground state of $\mathrm{Si}_{2}$, and the ${ }^{2} \Pi_{u}$ state was approximately $250 \pm 80 \mathrm{~cm}^{-1}$ higher in energy). All of the low-lying triplet and singlet valence states of $\mathrm{Ge}_{2}$ can in principle be accessed in the PES by photodetaching an electron from the valence orbitals of at least one of the anion states. Figure (8.1) shows a schematic of the low-lying neutral and anion electronic states including the various $\Omega$ components, and the one-electron transitions between them. For reference, the transition observed by Weltner is also shown as the darker arrow connecting the ${ }^{3} \Sigma_{g}{ }^{-}\left(\mathrm{XO}_{g}{ }^{+}\right)$and ${ }^{3} \Pi_{u}\left(1_{u}\right)$ 
neutral leveis. Two-electron transitions are generally too weak to be observed using our particular photodetachment techniques. For instance, the transition from the ${ }^{2} \Sigma_{g}+\left(\sigma_{g}{ }^{1} \pi_{u}{ }^{4}\right)$ anion state to either component of the ${ }^{3} \Sigma_{g}-\left(\sigma_{g}{ }^{2} \pi_{u}{ }^{2}\right)$ neutral would require both electron detachment and rearrangement, and will be too weak to be observed in either the PES or ZEKE spectrum.

While any of the 1-electron transitions in Figure (8.1) can be observed in the PES, the selection rules in ZEKE spectroscopy are more restrictive due to the Wigner threshold law. ${ }^{18}$ For atomic systems, this dictates that near threshold, the detachment cross section goes as:

$$
\sigma \propto \sigma_{0}\left(E_{h v}-E_{\text {threshold }}\right)^{l+1 / 2}
$$

where $\left(E_{h v}-E_{\text {threshold }}\right)$ and $\ell$ are the kinetic energy and angular momentum of the ejected electron, respectively. Geltman and coworkers extended the threshold law to diatomics with the same result. ${ }^{19}$ From Eqn. (8.1) it can be seen that the cross section rises sharply above threshold only for $\ell=0$, or s-wave electrons. As ZEKE spectroscopy relies an detecting electrons near a detachment threshold, it is sensitive only to s-wave transitions. Given the angular momentum of ortitais in a homonuclear diatomic, the end result is that only transitions involving the detachment from $\pi_{u}$ or $\sigma_{u}$ orbitals yield s-wave photoelectrons. On Figure (8.1), thase $\ell>0$ transitions that cannot be observed using ZEKE are indicated as dotted lines.

While at times inconvenient, the more restrictive ZEKE sefection rules can be useful in the situation where the PES is congested by overlapping transitions, as is the case with both $\mathrm{Si}_{2}$ and $\mathrm{Ge}_{2}{ }^{-}$. In addition, it effectively reveals the orbital from which an electron is ejected in a particular transition, which greatly facilitates spectral assignment. From the $\mathrm{Ge}_{2}-\mathrm{PES}$ and ZEKE spectrum, the splitings (or term energies) between the $\Omega$ components are resolved for both the ${ }^{3} \Sigma_{g}$ and ${ }^{3} \Pi_{u}$ states, and the term energies for the ${ }^{1} \Delta_{g},(1)^{1} \Sigma_{g}+$ and ${ }^{1} \Pi_{u}$ states are extracted. Vibrational frequencies for all of these states are determined from the ZEKE 
spectrum. Additionally, the relative energies and vibrational frequencies of the ${ }^{2} \Pi_{u}(3 / 2)$ and the $2 \Sigma_{\mathrm{g}}{ }^{+}$anion states are also determined.

\section{Experimental}

The apparatus used to obtain the ZEKE spectrum of $\mathrm{Ge}_{2}^{-}$has been described in detail elsewhere, but the basic operation is as follows. A beam of cold germanium clus.ers is generated in a molecular beam source similar to that developed by Smalley.20 The surface of a rotating and translating gemanium rod is ablated using $6 \mathrm{~mJ} / p u l s e$ of the second hamonic output of a Nd:YAG laser operated at a $20 \mathrm{~Hz}$ repetition rate. The resulting plasma is then entrained in a pulse of $90 \% \mathrm{Ne} / 10 \%$ He carrier gas from a piezoelectric valve, ${ }^{21}$ typically with a barking pressure of 60 to 70 psi. In order to generate sufficient quantities of $\mathrm{Ge}_{2}$, the source was run under conditions that generally produce vibrationally hot ions (e.g., lower beam pulse duration, earlier rod ablation selative to camer gas entrainment), as the colder conditions tend to skew the mass distribution to higher mass. The regatively charged species in the subsequent expansion that pass through a $2 \mathrm{~mm}$ skimmer are colinearly accelerated to $1 \mathrm{keV}$, and massselected using time-of-flight. ${ }^{22}$

The mass-separated anions then enter a detector region where they are selectively photodetached using an excimer-pumped tunable dye laser. As the dye laser is scanned through the detachment continuum of the ion of interest, only those photoefectrons ejected with nearly zero kinetic energy (ZEKE's) in the frame of the molecular beam are collected as a function of detachınent energy. The resulting spectrum is therefore peaked at the thresholds of transitions from anion to neutral levels. This selective detection of threshold electrons, based on techniques developec: by Müller-Dethlefs et al. ${ }^{23}$ for the photoionization of neutrals, yields an energy resolution of $3 \mathrm{~cm}^{-1}$. 
Tha electron signal for the ZEKE spectrum of $\mathrm{Ge}_{2}{ }^{*}$ was signal averaged for 1500 shots/point, and normalized to the ion current and detachment laser power. The dyes used were Rhodamine 610, Rhodamine 590, Coumarin 540A. Coumarin 503, Coumarin 480, and Coumarin 450 .

\section{iII. Results}

The PES of $\mathrm{Ge}_{2}-$ obtained using $2.98 \mathrm{eV}$ photon energy ( $416 \mathrm{~nm}$ ) and at two laser polarizations is shown in Figure (8.2) (dottfod lines). Photoelectrons ejected from diterent orbitals will generally haye different angular distributions, and by comparing the two different laser polarizations, the extent of the overiapping transitions conyesting the PES can be seen. Moreover, by comparing how the peak intensities change with the detachment laser polarization, a first-order idea of which transitions involve p-wave detachment can be obtained; in the similarly obtained PES of $\mathrm{Si}_{2}$, the p-wave transitions tended to be more intense in the $\theta=0^{\circ}$ (electron detection parallel to the electric field vector of the detachment laser) spectrum. For instance, peaks $C_{\Omega}$ and peak $F$ are particularly intense in the $\theta=0^{\circ}$ spectrum, suggesting they involve p-wave detachment. The group of peaks at the lower-energy end of the spectrum are comprised of transitions to the lowest-lying triplet states of the neutral, and the group of peaks on the high-energy end are the Iransitions to the higher-lying singlet neutral states. The $\mathrm{Ge}_{2}$ ' ZEKE ipectrum (solid line) has been superimposed onto the $\theta=9 G^{\prime}$ PES. Note that the PES have been placed on the ZEKE binding energy $\left(\mathrm{cm}^{-1}\right)$ scale.

The upper, solid trace in Figure (8.3) shows the triplet band $0^{\prime}$ the ZEKE spectrum on an expanded scale. The peak labeling convention is based on the assignments that will be discussed in detail later. Briefly, different letters correspond to different electronic bands, and wher: there are two subscripts, the first denotes the value of $\Omega$ of the neutral state, and the second is the vibrational quanta in the neutral state (in the singlet band. snly the neutral 
vibrational quanta is denoted in the peak label suascripts). The triplet band is dominated by a progression of doublets labeled $A_{0, v}$ and $A_{1, v}(v=0,1,2,3)$ which have an average splitting of $117 \pm 5 \mathrm{~cm}^{-1}$. The centers of the doublets are spaced by $286 \pm 5 \mathrm{~cm}^{-1}$; this is presumainly a viorational progression. The lower-intensity peaks $a_{0, c}$ and $a_{1,0}$ appear to be transitions from the furdamental of the anion (hot bands), and ara found $3 c 9: 5 \mathrm{~cm}^{-1}$ to tower photon energy of peaks $A_{0,0}$ and $A_{1,0}$, respectively. In addition to these features, there are lower intensity peaks spaced irregularly among the $286 \mathrm{~cm}^{-1}$ progression. The most distinct of these is $\mathrm{B}_{2,0}$ found 58 $\pm 7 \mathrm{~cm}^{-1}$ to higher photor, energy of $A_{0,0}$. Peak $b_{2,0}$ also appears to be a hot hand spaceu $326 \pm$ $10 \mathrm{~cm}^{-1}$ to icwer photon energy of peak $t_{2,0}$. There are also shoulders en both sides of peak $A_{1,1}$ found $308 \pm 10$ and $37^{A} \pm 10 \mathrm{~cm}^{-1}\left(B_{2,1}\right.$ and $B_{1,0}$, respectively) to higher photon energ. or peak $B_{2,0}$. Peaks $B_{0,0}$ and $B_{0+, 0}$, while appearing to be a part of the $A_{0, v}$ and $A_{1 . v}$ progression, are actually spaced $340 \pm 10 \mathrm{~cm}^{-1}$ rather than $286 \mathrm{~cm}^{-1}$ from peaks $A_{0.2}$ and $A_{1,2}$. (peak $A_{0.3}$, found $80 \mathrm{~cm}^{-1}$ to luwer phrten energy of peak $B_{0,0,0}$, is the lail of the $A_{v, v}$ progression). There are several transitions that are apparent in the [PES [Figure (8.2)] that are absent in this energy Prion of the ZEKE spectndm. All of the peaks labeled " $C_{x}{ }^{4}$ in the upper panel of Figure (8.2) should be found within $100 \mathrm{~cm}^{-1}$ of $17100 \mathrm{~cm}^{-1}\left(\mathrm{C}_{2 u}\right) .17460 \mathrm{~cm}^{-1}\left(\mathrm{C}_{1 \mathrm{u}}\right)$, and $18050 \mathrm{~cm}^{-1}$ $\left(C_{04}\right)$. Peak positions and relative energies are sımmanized in Table (8.1). The peak positions generally have an uncertainty of $\pm 5 \mathrm{~cm}^{-1}$, and so the relative energies are generally good to \pm 7 $\mathrm{cm}^{-1}$.

The ZEKE spectnum of the singlet band of $\mathrm{Ge}_{2}^{-}$is shown on an expended scale as the solid trace in Figure (8.4). There appear io be three electrenic transitions contributing to the structure in this region. The lowest energy is a $276 \pm 3 \mathrm{~cm}^{-1}$ priugression labeled $D_{v}(v=0, \ldots$, 4). The origin, $D_{0}$, which is actually a partially-resolved doublet. is found $\$ 314 \mathrm{~cm}^{-1}$ above peak $A_{0,0}$ in the singlet band. Feak $d_{0}$ appears to be a hot band, and is found $307 \pm 7 \mathrm{~cm}^{-1}$ to lower photon erergy of $D_{0}$. E $E_{0}$ indicates the origin of a second electronic transition found $4664 \mathrm{~cm}^{-1}$ to higher photon energy of peak $A_{0,0}$. Peak $G_{0}$, is spaced approximately $280 \mathrm{c:n} \mathrm{n}^{-1}$ to higher 
photon energy of peak $E_{0}$, which could reasonably be a vibrational spacing. However, peak $E_{2}$, which is found $607 \pm 7 \mathrm{~cm}^{-1}$ from $E_{0}$, is much less intense than would be expected if $E_{0}, G_{0}$ and $E_{2}$ formed a progression. On the other hand, peak $G_{0}$ is broad, and it is feasible that two transitions are contributing to $G_{0}$ : the fundamental of $E_{0}$, and yet another electronic transition for which $G_{1}$ is the fundamental. This gives a frequency of $303 \pm 5 \mathrm{~cm}^{-1}$ for the neutral state accessed by the $E$ transition, and $204 \pm 7 \mathrm{~cm}^{-1}$ for the state accessed by the $G$ transition. Peak $\mathrm{e}_{0}$, another hot band, is $329 \pm 7 \mathrm{~cm}^{-1}$ from $\mathrm{E}_{0}$. Peaks $\mathrm{g}_{1}$ and $\mathrm{g}_{2}$ are suaced approximately 100 $\mathrm{cm}^{-1}$ to lower photon energy of $\mathrm{G}_{0}$ and $\mathrm{G}_{1}$, respectively. As the anion frequencies so far have been either approximately $309 \mathrm{~cm}^{-1}$ or $326 \mathrm{~cm}^{-1}$, peaks $g_{1}$ and $g_{2}$ are spaced appropriately to be the $v^{\prime}=1 \leftarrow v^{\prime \prime}=1$ and $v^{\prime}=2 \leftarrow v^{\prime \prime}=1$ sequence and hot band transitions, respectively, between an anion state and the neutral state responsible for $G_{0}$. Again, Table (8.1) summarizes peak positions and relative energies.

By comparing the singlet bands in the ZEKE and PES spectra, there appears to be a one to one correspondence between the transitions in both spectra. However, as mentioned above, peak $F$ in the PES appears to be a p-wave transition based on its polarization dependence. Upon close inspection of peak $F$ in the lower panel of Figure (8.1), the overlapping ZEKE peak $\left(G_{0}\right)$ falls on the higher-energy side. It therefore seems likely that peak $F$ is composed of two transitions, one of which procetds via s-wave, and the other via p-wave.

\section{Analysis and Discussion}

The assignment and subsequent analysis of the spectra requires consideration of both the ZEKE spectra and the PES. The approach taken bolow is to first analyze the triplet band of the PES and ZEKE spectrum considering the higher-resolution spectroscopic information from Weltner's matrix studies. From this, the spectroscopic constants of the anion states will be 
extracted. With more information about the anion states, the analysis of the singlet band then proceeds in a more straightforward manner.

\section{A. Triplet band}

Figure (8.1) illustrates the three one-electron transitions between the doublet anion states and the lowest-lying triplet neutral states, two of which should be observed in the ZEKE spectrum [Figure (8.3)]: the ${ }^{3} \Sigma_{g}\left(1_{g}, \mathrm{X0}_{g}{ }^{+}\right) \leftarrow{ }^{2} \Pi_{u}(3 / 2)$ transition and the ${ }^{3} \Pi_{u}\left(0^{+}, 0,1_{u}, 2_{u}\right) \leftarrow$ $2 \Sigma_{g}+$ transitions. We can immediately assign peaks $A_{\Omega, v}$ to the former. As pointed out by Weltner, ${ }^{10}$ the zero field splitting (ZFS) between the $\mathrm{XO}_{\mathrm{g}}{ }^{+}$and $1_{\mathrm{g}}$ components of the neutral ${ }^{3} \Sigma_{\mathrm{g}}$ state of $\mathrm{Ge}_{2}$ is expected to be approximately $100 \mathrm{~cm}^{-1}$ based on the square of the relative spinorb:: splittings in the $\mathrm{Ge}$ and $\mathrm{Si}$ atoms $\left(\mathrm{SO}_{\mathrm{Ge}} / \mathrm{SO}_{\mathrm{Si}}=6.3\right)$ as well as the $\mathrm{ZFS}$ in $\mathrm{Si}_{2}\left(2.6 \mathrm{~cm}^{-1}\right)_{-}{ }^{24}$ It therefore seems reasonable to associate the $A_{0, v}-A_{1, v}$ doublet structure with the average 117 $\mathrm{cm}^{-1}$ spacing with the ZFS between the $\mathrm{XO}_{\mathrm{g}}{ }^{+}$and $1_{\mathrm{g}}$ components of the neutrai ${ }^{3} \Sigma_{\mathrm{g}}$ state. This splitting has contributions from both first order spin-spin interaction and second order spin-orbit interaction, but as the former is generally on the order of less than $1 \mathrm{~cm}^{-1}, 25117 \mathrm{~cm}^{-1}$ can be taken as the second-order spin-orbit constant (conventionally, $2 \lambda .50$ ). ${ }^{16}$ The $286 \mathrm{~cm}^{-1}$ vibrational frequency characteristic of the $A_{\Omega, v}$ progression is slightly higher than the 277 ( \pm-10 ; error bars were not included in the spectral analysis) $\mathrm{cm}^{-1}$ ground state frequency determined from Raman matrix studies. ${ }^{17}$ From the position of the hot bands (peaks $a_{0,0}$ and $a_{9,0}$ ) we determine that the frequency of the ${ }^{2} \Pi_{U}$ anion is $309 \mathrm{~cm}^{-1}$. From the $S_{2}-Z E K E$ spectrum, we were also able to determine the $122 \mathrm{~cm}^{-1}$ spin-orbit splitting in the ${ }^{2} \Pi_{U}$ anion, ${ }^{1}$ as the $\Omega=1 / 2$ level was sufficiently populated in the beam in order to observe both the ${ }^{3} \Sigma_{g} \leftarrow^{2} \Pi_{u}(3 / 2)$ and the ${ }^{3} \Sigma_{g}{ }^{-} \leftarrow{ }^{2} \Pi_{u}(1 / 2)$ transitions. However, since the $\mathrm{Ge}_{2}-$ spin-orbit splitting is expected to be roughly 700 to 800 $\mathrm{cm}^{-1}$, the population of spin-orbit excted anions in the beam is likely to be very small, and we only observe transitions from the $\Omega=3 / 2$ component. 
We can simulate the vibrational structure in the ${ }^{3} \Sigma_{g}-\left(0_{g}+1_{g}\right) \leftarrow 2 \Pi_{u}(3 / 2)$ transitions within the Franck-Condon approximation. In the simulation, we assume that the intensity of individual vibronic transitions within an electronic band are proportional to their Franck-Condon factors (FCF's),

$$
\text { Intensity } \propto\left|\left\langle v^{\prime} \mid v^{\prime \prime}\right\rangle\right|^{2}
$$

The vibrational wave functions of the neutral and anion, $v^{\prime}$ and $v^{\prime \prime}$, respectively, are taken to be harmonic (or Morse) oscillators with the frequencies determined from the spectra. The relative: bond distances of the anion and neutral are then varied until the extension of the simulated progression matches the observed progression.

The simulation of the ${ }^{3} \Sigma_{\mathrm{g}}\left(0_{\mathrm{g}}{ }^{+}, 1_{\mathrm{g}}\right) \leftarrow{ }^{2} \Pi_{u}(3 / 2)$ transitions is shown as the lighter dashed trace on the lower part of Figure (8.3). Harmonic oscillator wave functions with 309 and $286 \mathrm{~cm}^{-1}$ frequencies were used for the anion and neutral, respectively. A bond distance difference of $0.059 \pm 0.003 \AA$ was required to reproduce the extension of the piugression, and while the direction of the bond length change cannot be determined from the simulation (using harmonic oscillators) the anion bond length should be shorter than the neutral, as the transition involves removing a bonding electron. An anion vibrational temperature of $350 \mathrm{~K}$ was required to match the hot band intensity; this temperature is fairly typical for our cluster source given this vibrationai frequency.

Given the ${ }^{3} \Sigma_{g}\left(0_{g}{ }^{+}, 1_{g}\right) \leftarrow{ }^{2} \Pi_{u}(3 / 2)$ assignment and the term energy of the ${ }^{3} \Pi_{u}\left(1_{u}\right)$ state from Weltner's matrix studies, we would expect the ${ }^{3} \Pi_{u}\left(1_{u}\right) \leftarrow{ }^{2} \Pi_{u}(\xi / 2)$ transition to lie approximately $700 \mathrm{~cm}^{-1}$ above the ${ }^{3} \Sigma_{\mathrm{g}}\left(\mathrm{XO}_{\mathrm{g}}{ }^{+}\right) \leftarrow{ }^{2} \Pi_{\mathrm{u}}(3 / 2)$ transition, and to be observed only in the PES as it involves the removal of a $\sigma_{\mathrm{g}}$ electron. In the upper panel of Figure (8.2), peak $\mathrm{C}_{1 \mathrm{u}}$ lies $670 \pm 50 \mathrm{~cm}^{-1}$ to higher energy than peak $A$. We therefore assign peak $C_{1 u}$ in the PES to the ${ }^{3} \Pi_{u}\left(1_{u}\right) \leftarrow{ }^{2} \Pi_{u}(3 / 2)$ transition. It then follows that peak $C_{2 u}$ found $360 \mathrm{~cm}^{-1}$ to lower 
energy, is the ${ }^{3} \Pi_{\perp}\left(2_{u}\right) \leftarrow{ }^{2} \Pi_{u}(3 / 2)$ transition. From the PES, it is not clear which of the lowerintensity transitions to slightly higher energy are the ${ }^{3} \Pi_{u}\left(0_{u}\right) \leftarrow{ }^{2} \Pi_{u}(3 / 2)$ and ${ }^{3} \Pi_{u}\left(0_{u}{ }^{+}\right) \leftarrow{ }^{2} \Pi_{u}$ (3/2) transitions.

We can now apply what is known about the ${ }^{3} \Pi_{u}\left(2_{u}-1_{u}\right)$ splitting to interpret the remaining structure in the triplet region of the ZEKE spectrum that has not been accounted for by the ${ }^{3} \Sigma_{g}-\left(X_{g^{+}}{ }^{+}{ }^{1}\right) \leftarrow{ }^{3} \Pi_{u}(3 / 2)$ transition. Peaks $B_{\Omega, v}$ must result from the ${ }^{3} \Pi_{u}\left(0_{u}{ }^{+}, 0_{u}, 1_{u}\right.$ $\left.2_{u}\right) \leftarrow 2 \Sigma_{g}+$ transitions, the only others that proceed via s-wave detachment in this energy region. By companing the dotted trace of the simulation with the ZEKE spectrum, the lowerintensity peaks and shoulders labeled $\mathbf{B}_{\mathbf{\Omega}, \mathrm{v}}$ stand out more clearly as a second set of electronic transitions. The most distinct of these peaks is $B_{2,0}$. The shoulder $B_{1,0}$ is found $374 \mathrm{~cm}^{-1}$ to higher photon energy, which is close to the ${ }^{3} \Pi_{u}\left(2_{u}-1_{U}\right)$ splitting observed in the PES. Moreover, $B_{2,1}$ and $B_{1,1}$ are found approximately $308 \mathrm{~cm}^{-1}$ to higher photon energy of $B_{2.0}$ and $B_{1,0}$, respectively, which is the vibrational frequency of the ${ }^{3} \Pi_{u}\left(1_{u}\right)$ state determined by Weltner. We therefore assign peak $B_{2,0}$ to the ${ }^{3} \Pi_{u}\left(2_{u}\right) \leftarrow{ }^{2} \Sigma_{g}+$ transition, and $B_{1,0}$ to the ${ }^{3} \Pi_{u}\left(1_{u}\right) \leftarrow{ }^{2} \Sigma_{g}+$ transition. Peaks $B_{2,1}$ and $B_{1,1}$ are transitions to the fundamental levels of the ${ }^{3} \Pi_{v}\left(2_{v}\right)$ and ${ }^{3} \Pi_{v}$ $\left(1_{\mathrm{u}}\right)$ states, respectively.

From the position of the ${ }^{3} \Pi_{v}\left(t_{u}\right) \leftarrow{ }^{2} \Sigma_{g}+$ transition (peak $B_{1,0}$ ), the relative energy of the ${ }^{2} \Sigma_{g}+$ and ${ }^{2} \Pi_{u}(3 / 2)$ anion states can be determined. The ${ }^{3} \Pi_{u}\left(1_{u}\right) \leftarrow{ }^{2} \Sigma_{g}+$ transition is $432 \pm 10$ $\mathrm{cm}^{-1}$ higher in energy than the ${ }^{3} \Sigma_{g}\left(\mathrm{XO}_{\mathrm{g}}{ }^{+}\right) \leftarrow{ }^{2} \Pi_{u}(3 / 2)$ transition, but from Weltner's work, the $3 T_{u}\left(1_{u}\right)$ neutral zero point level is known to lie $711 \pm 3 \mathrm{~cm}^{-1}$ above the ${ }^{3} \Sigma_{g}-\left(\mathrm{Ko}_{\mathrm{g}}{ }^{+}\right)$zero point level. ${ }^{10}$ This gives that for the anion, the ${ }^{2} \Pi_{v}(3 / 2)$ level is $279 \pm 11 \mathrm{~cm}^{-1}$ below the ${ }^{2} \Sigma_{g}+$ level (by using Weltner's value, the energy splitting we determine for the anion states is more accurate than what we would have determined from comparing the position of peak $\mathrm{C}_{2 u}$ in the PES and peak $B_{2,0}$ in the ZEKE spectrum, which would have yielded $240 \pm 80 \mathrm{~cm}^{-1}$ ). The relative weakness of the $B$ transitions likely results from their originating from an excited anion level. Given the expected spin-orbit splitting in the ${ }^{2} \Pi$ state of $\mathrm{Ge}_{2}$, the ${ }^{2} \Pi_{u}(1 / 2)$ excited level 
may be roughly 400 to $500 \mathrm{~cm}^{-1}$ above the $2 \Sigma_{g}+$ state. The frequency of the ${ }^{2} \Sigma_{g}+$ can be extracted from the position of the $b_{2,0}$ hot band, which is found $326 \mathrm{~cm}^{-1}$ to lower photon energy of peak $B_{2,0}$. This is slightly higher than the $309 \mathrm{~cm}^{-1}$ frequency determined for the ${ }^{2} \Pi_{u}(3 / 2)$ state.

So far, peaks $\mathrm{B}_{0-, 0}$ and $\mathrm{B}_{0+, 0}$ have not been addressed. These are approximately half the intensity of peak $B_{2,0}$, and they are spaced 856 and $968 \mathrm{~cm}^{-1}$, respectively, to higher photon energy. As there are no other electronic transitions that should be observed in this region of the spectrum, we tentatively assign them to the ${ }^{3} \Pi_{u}\left(0_{u}{ }^{-}\right) \leftarrow{ }^{2} \Sigma_{g}{ }^{+}$and ${ }^{3} \Pi_{u}\left(0_{u}+\right) \leftarrow{ }^{2} \Sigma_{g}+$ transitions. It should be noted again that although the spacing between peaks $B_{0+, 0}$ and $B_{0-.0}$ is very close to the $A_{0, v}-A_{1, v}$ splitting, peaks $B_{0+, 0}$ and $B_{0,0}$ do not energetically fall in the $A_{0, v}-A_{1, v}$ vibrational progression, and perturbation of this progression spacing is unlikely given the absence of any other close-lying gerade states. If these peaks do represent the ${ }^{3} \Pi_{u}\left(0_{u} \pm\right) \leftarrow$ ${ }^{2} \Sigma_{g}+$ transitions, the spin coupling constant, $A$, for the ${ }^{3} \Pi_{u}$ state is between $856 / 2=428$ to $968 / 2$ $=484 \mathrm{~cm}^{-1}$, which is higher than the $1_{\mathrm{U}}-2_{\mathrm{U}}$ splitting $\left(374 \mathrm{~cm}^{-1}\right)$. The asymmetric arrengement of the ${ }^{3} \Pi_{u}\left(0_{u}^{ \pm}\right)$and ${ }^{3} \Pi_{u}\left(\eta_{u}\right)$ states about the ${ }^{3} \Pi_{u}\left(\boldsymbol{1}_{u}\right)$ state was also observed for the $\mathrm{Si}_{2}{ }^{3} \Pi_{u}$ state in Weltner's studies, and is due to the mixing of the $\Omega=1$ level with the higher-lying ${ }^{1} \Pi_{u}$ state. This mixing will be further addressed in the next section where term energy of the ${ }^{1} \Pi_{u}$ state is discussed.

The ${ }^{3} \Pi_{u}\left(O_{u}{ }^{+}\right)$level is always higher-lying than the ${ }^{3} \Pi_{u}\left\langle 0_{u}{ }^{-}\right)$level due to the form of the (Off-diagonal) spin-spin interaction. ${ }^{15}$ The splitting effectively arises because th.: ${ }^{3} \Pi_{u}\left(0_{u}^{-}\right)$state correlates to the $\mathrm{Ge}\left({ }^{2} \mathrm{P}_{3 / 2}\right)+\mathrm{Ge}\left({ }^{2} \mathrm{P}_{3 / 2}\right)$ dissociation asymptote, while the higher-lying ${ }^{3} \Pi_{u}\left(\mathrm{O}_{\mathrm{u}}{ }^{+}\right)$ level correlates to the $\mathrm{Ge}\left({ }^{2} \mathrm{P}_{1 / 2}\right)+\mathrm{Ge}\left({ }^{2} \mathrm{P}_{3 / 2}\right)$ asymptote. Mloreover, the ${ }^{3} \Pi_{\mathrm{u}}\left\langle\mathrm{O}_{\mathrm{u}}{ }^{+}\right)$potential necessarily crosses the ${ }^{3} \Sigma_{U^{+}}+\left(0_{U^{+}}\right)$and ${ }^{3} \Sigma_{L^{-}}\left(0_{u^{+}}\right)$potentials near the dissociation continuum, which can give rise to further electronic coupling. The magnitude of this splitting in any diatomic is rarely measured, singe transitions to only one of the leveis is generally allowed from a particular state. However, calculations on $\ln _{2}$ give a $\mathrm{O}_{\mathrm{u}^{+}}{ }^{+} \mathrm{O}_{\mathrm{u}}^{-}$splitting of $231 \mathrm{~cm}^{-1}$ with a 
calculated $2 A$ of $1397 \mathrm{~cm}^{-1}$, and for $S n_{2}$, the $\mathrm{O}_{\mathrm{u}}{ }^{+} \mathrm{O}_{\mathrm{u}}^{-}$splitting is calculated to be $108 \mathrm{~cm}^{-1}$ with $2 A$

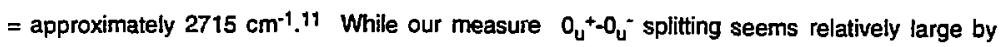
comparison to the calculated $\mathrm{O}_{\mathrm{u}}{ }^{+} \mathrm{O}_{\mathrm{u}}$, splitting for isoelectronic $\mathrm{Sn}_{2}$, the order of magnitude seams reasonable. Nonetheless, the similarity between the $\mathrm{Ge}_{2} \mathrm{O}_{\mathrm{u}}{ }^{+}-\mathrm{O}_{\mathrm{u}}-$ and $\mathrm{XO}_{\mathrm{g}}{ }^{+-1} \mathrm{~g}$ splittings compels us view this whole issue with some caution.

As can be seen in the lower half of Figure (8.3), a spectral simulation was also performed for the ${ }^{3} \Pi_{u}\left(0_{u}+0_{u}, 1_{u}, 2_{u}\right) \leftarrow{ }^{2} \Sigma_{g}+$ transitions (heavier dashed line). The neutral frequency assumed for all four components was $308 \mathrm{~cm}^{-1}$, per Weltner, ${ }^{10}$ and the anion frequency was assumed to be $326 \mathrm{~cm}^{-1}$. The transitions to the ${ }^{3} \Pi_{u}\left(0_{u}^{ \pm}\right)$states were assumed to be half the intensity of the transitions to the ${ }^{3} \Pi_{u}\left(1_{u}\right)$ and ${ }^{3} \Pi_{u}\left(2_{u}\right)$ states, which were in turn scaled to have 0.25 the intensity of the ${ }^{3} \Sigma_{g}\left(\mathrm{XO}_{g}{ }^{+}\right) \leftarrow{ }^{2} \Pi_{U}(3 / 2)$ transition in order to best match the spectrum. A bond length difference of $0.04 \AA$ between the anion and neutral was assumed to match the extension of the vibrational progression, and again, the anion should have the shorter bond distance.

Geometry calculations on the lowest-lying neutral states predict the bond length of the ${ }^{3} \Pi_{u}$ excited state to be $0.1 \mathrm{~A}$ shorter than the ${ }^{3} \Sigma_{g} \cdot$ state, $11,12,13,14$ which, considering the bond length differences determined from the spectra, suggests that the anion ${ }^{2} \Pi_{\lrcorner}$state is approximate!y $0.08 \dot{A}$ longer than the ${ }^{2} \Sigma_{g}{ }^{+}$state (calculations yield values from 2.42 to $2.46 \AA$ for the ${ }^{3} \Sigma_{g}$ - state and 2.33 to $2.39 \mathrm{~A}$ for the ${ }^{3} \Pi_{u}$ state). Thus, shifting an electron from the $\sigma_{g}$ to the $\pi_{u}$ orbital results in shortening the bond by nearly $0.1 \AA$ in both the anion and neutral. The relative bond distances between the anion and neutral states are startlingly similar to those found for $\mathrm{Si}_{2}$ and $\mathrm{Si}_{2}$ - The anion states were found to be different by $0.09 \dot{A}$ with the ${ }^{2} \Pi_{u}$ state being longer, and the neutral states were known to be diffierent by $0.09 \dot{A}$ with the ${ }^{3} \Sigma_{g}$ state being longer. Moreover, spectral simulations of the ZEKE spectrum of $\mathrm{Si}_{2} \cdot$ gave bond length differences on the order of $0.04 \AA$ in both ZEKE transitions. This interesting similarity between 
the response of the bond lengths of $\mathrm{Ge}_{2}$ and $\mathrm{Si}_{2}$ to the shifting of valence electrons within the triplet manifold further reflects the electronic similarity between these two species.

Table (8.1) summanizes the peak assignments discussed in this section, and Table (8.2) summarizes the spectroscopic constants extracted from the spectrum and used in the spectrai simulations, including the term energies of the ${ }^{3} \Pi_{u}\left(0_{u}+, 0_{u}, 1_{u}, 2_{u}\right)$ states applied in the spectra! simulation.

\section{B. Singlet band}

From electronic state calculations, ${ }^{11}$ transitions to the ${ }^{1} \Delta_{g},(1)^{t} \Sigma_{g}+$ and ${ }^{1} \Pi_{u}$ neutral states lie roughly $0.5-0.7 \mathrm{eV}$ above the ground state should result in the structure observed to higher energy in both the PES. In Table (8.2), the ab initio tem energies of these three states are listed. Figure (8.1) shows the four 1-electron transitions between the two anion states and these three neutral states. An additional $(2)^{1} \Sigma_{\mathrm{g}}{ }^{+}$state is predicted to lie approximately $1.3 \mathrm{eV}$ above the ground state; the $(2)^{1} \Sigma_{g}+{ }^{2} \Sigma_{g}+(p$-wave) transition is not observed in the PES obtained using $2.98 \mathrm{eV}$ photon energy, but in the PES obtained using $4.66 \mathrm{eV}$ photon energy, it was found to lie (with lower accuracy) $9300 \pm 100 \mathrm{~cm}^{-1}\left(1.15 \mathrm{eV}\right.$ ) above the ${ }^{3} \Sigma_{\mathrm{g}} \leftarrow^{-2} \Pi_{u}(3 / 2)$ transition. Adding the term energy of the ${ }^{2} \Sigma_{g}{ }^{+}$anion state yields $T_{e}=9580 \mathrm{~cm}^{-1}$ for the $(2)^{1} \Sigma_{g}{ }^{+}$ state. There are three transitions expected to be observed in the ZFKE spectrum: ${ }^{1} \Delta_{g} \leftarrow{ }^{2} \Pi_{y}$ (3/2), (1) ${ }^{1} \Sigma_{g}{ }^{+} \leftarrow{ }^{2} \Pi_{u}(3 / 2)$, and ${ }^{1} \Pi_{u} \leftarrow{ }^{2} \Sigma_{g}+$

Since the ${ }^{1} \Delta_{g}$ state is predicted to be the lowest-lying of the singlet states, peaks $D_{\psi}(v=$ $0, \ldots, 4)$ are assigned to the ${ }^{1} \Delta_{g}\left(v^{\prime}=0, \ldots, 4\right) \leftarrow{ }^{2} \Pi_{u}(3 / 2)$ transition. The $276 \pm 3 \mathrm{~cm}^{-1}\left(\omega_{\mathrm{e}} \chi_{\mathrm{e}}=\right.$ $1.2 \pm 0.6 \mathrm{~cm}^{-1}$ ) progression is the most extended of all the progressions. This is consistent with calculated geometries, which predict the ${ }^{1} \Delta_{\mathrm{g}}$ state to have the longest tond distance of all the low-lying states. The position of the hot band, $d_{0}$, gives an anion frequency of approximately $308 \mathrm{~cm}^{-1}$, which further supports that this is a transition from the ${ }^{2} \Pi_{u}(3 / 2)$ anion state. The 
energy spacing between peak $D_{0}$ and $A_{0,0}$ directly gives the term energy of the ${ }^{1} \Delta_{g}$ state: 3314 $\pm 7 \mathrm{~cm}^{-1}$.

As with the triplet band, the Franck-Condon simulation gives an approximate bond length difference between the anion and neutral. The total simuiation of the singlet band is shown as the dashed line in Figure (8.4). In the case of the transition to the ${ }^{1} \Delta_{g}$ state, the bond length difference is determined to be $0.095 \pm 0.005 \AA$. This further suggests that the bond distance in the ${ }^{1} \Delta_{\mathrm{g}}$ state is $0.036 \mathrm{~A}$ longer than that in the ${ }^{3} \mathrm{\Sigma g}_{\mathrm{g}}\left(\mathrm{XO}_{\mathrm{g}}{ }^{+}\right)$state. This is in qualitative agreement with the calculated bond distance between these two states, $0.06 \AA .{ }^{11}$

Again referring to the calculated frequencies of the singlet states [Table (8.2)], the (1) $1 \Sigma_{g}+$ state has a substantially lower frequency than the other singlet states. We therefore assign the $G_{0}-G_{1}$ progression to the $(1)^{1} \Sigma_{g}+\leftarrow{ }^{2} \Pi_{u}(3 / 2)$ transition, as it exhitits the smallest vibrational spacing, $204 \mathrm{~cm}^{-1}$. From the average spacing of the sequence bands, $g_{1}$ and $g_{2}$, the anion frequency in this transition is approximately $305 \mathrm{~cm}^{-1}$, which is close to the previously determined ${ }^{2} \Pi_{u}(3 / 2)$ frequency, further supporting the assignment. As above, the term energy of this state can be extracted from the energy spacing between peaks $G_{0}$ and $A_{0,0}: 4941 \mathrm{~cm}^{-1}$. In the simulation shown, a bond length difference of $0.045 \pm 0.005 A$ was found to satisfactorily fit the progression. This is $0.014 \dot{A}$ less than that used in the ${ }^{3} \Sigma_{g}{ }^{-}\left(\mathrm{XO}_{g}{ }^{+}\right) \leftarrow{ }^{2} \Pi_{u}(3 / 2)$ fit, suggesting that the bond distances of the ground $3 \Sigma_{g}\left(\mathrm{XO}_{\mathrm{g}}{ }^{+}\right)$and excited $(1)^{1} \Sigma_{g^{+}}$states have nearly identical bond distances. While this is counterintuitive given the lower frequency in the excited singlet state, geometry calculations do predict these two states to have nearly identical bond lengths, presumably because the $(1)^{1} \Sigma_{g}{ }^{+}$is a mixture of both the $1 \pi_{u}{ }^{4}$ and $2 \sigma_{g}{ }^{2} 1 \pi_{u}{ }^{2}$ configurations, and population in the $1 \pi_{\mathrm{u}}$ orbital results in a shorter bond distance. ${ }^{11,12,13,14}$

The remaining vibrational progression in the ZEKE spectrum, $E_{\mathrm{y}}$, can be assigned by elimination to the ${ }^{1} \Pi_{u} \leftarrow{ }^{2} \Sigma_{g}+$ transition. The position of the hot band, $e_{0}$, gives an anion frequ. ency of $329 \pm 5 \mathrm{~cm}^{-1}$, which is in agreement with the $326 \pm 5 \mathrm{~cm}^{-1}$ determined for the ${ }^{2} \Sigma_{\mathrm{g}}{ }^{+}$ 
anion state determined from the triplet band. In the fit shown, the neutral frequency was chosen as $304 \pm 5 \mathrm{~cm}^{-1}$ with a bond length change between the anion and neutral of $0.05 \pm 0.005 \dot{A}$.

In order to determine the term energy of the ${ }^{1} \Pi_{U}$ state, we must add the term energy of the ${ }^{2} \Sigma_{g}{ }^{+}$anion state to the energy separation between peak $E_{0}$ and $A_{0,0}$, which gives a value of $4943 \mathrm{~cm}^{-i}$ ! Within the error bars of the experiment, we cannot assert which of the $(1)^{1} \Sigma_{g}{ }^{+}$and ${ }^{1} \mathrm{n}_{u}$ states is higher lying, and calculations do not give a strong indication; the $(1)^{1} \Sigma_{g}{ }^{+}$and the ${ }^{1} \Pi_{\mathrm{u}}$ states are predicted to close-lying, with Balasubramanian ${ }^{11}$ and Pacchioni ${ }^{13}$ predicting the ${ }^{1} \Pi_{\mathrm{u}}$ state to be higher-lying than the $(1)^{1} \Sigma_{g}+$ state, and Kingcade et al ${ }^{14}$ predicting the ${ }^{1} \Pi_{\mathrm{u}}$ state to be lower. While it may seem too remarkable a coincidence that these state are nearly degenerate, it is further supported by the sole p-wave transition observed in the singlot band of the PES [Figure (8.1)]. Peak $F$ in the PES, which can only be the ${ }^{1} \Pi_{u} \leftarrow{ }^{2} \Pi_{u}(3 / 2)$ transition, energetically coincides with peak $G_{0}$ in the ZEKE spectrum, which, in addition to irrefutably being an s-wave transition, has been definitively assigned to the $(1)^{4} \Sigma_{g}{ }^{+} \leftarrow{ }^{2} \Pi_{U}(3 / 2)$ transition. As the coincident transitions originate from the same anion state, the neutral states must coincide energetically.

Given the term energy of the $(1)^{1} \Sigma_{\mathrm{g}}{ }^{+}$state and the $\mathrm{ZFS}$ between the $\mathrm{XO}_{\mathrm{g}}{ }^{+}$and $1_{\mathrm{g}}$ levels of the ${ }^{3} \Sigma_{g}$ - state, an approximate value for the spin coupling constant, $A$, in the ${ }^{3} \Pi_{u}$ state can be calculated with, ${ }^{16}$

$$
2 \lambda^{\mathrm{SO}}=117 \mathrm{~cm}^{-1}=\frac{4 A^{2}}{E\left({ }^{1} \Sigma_{g}^{+}\right)-E\left({ }^{3} \Sigma_{g}^{-}\right)} .
$$

Adjusting the $T_{e}$ of the (1) ${ }^{1} \Sigma_{g}+$ state to account for the $117 \mathrm{~cm}^{-1}$ repulsion "felt" by both the (1) ${ }^{1} \Sigma_{g^{+}}$and $X_{\mathrm{g}^{+}}$levels, $2 A$ determined from Eqn. (8.2) is $742 \mathrm{~cm}^{-1}$, which is failly close to the ohserved splitting, $484 \mathrm{~cm}^{-1}$. 
The extent of the asymmetry between the ${ }^{3} \mathrm{I}_{u}\left(2_{u}, 1_{u}, 0_{u} \pm\right)$ levels similarly is due to the repulsion between the ${ }^{\dagger} \Pi_{u}$ and ${ }^{3} \Pi_{u}\left(1_{u}\right)$ levels, and can be calculated given the term energy of the ${ }^{1} \Pi_{U}$ state. The shitt to both states is gil'en by ${ }^{16} A^{2} / 2 K$, where $2 K$ is the depertumed splitting between the ${ }^{3} \Pi_{u}\left(1_{u}\right)$ and ${ }^{1} \Pi_{u}$ levels [i.e., the $\left({ }^{3} \Pi_{u}-{ }^{1} \Pi_{u}\right)$ exchange integral]. Assuming $A=$ $484 \mathrm{~cm}^{-1}$ from half the $B_{0+, 0}-B_{2.0}$ splitting, and $4000 \mathrm{~cm}^{-1}$ for the deperturted exchange, $A^{2} / 2 K$ is $60 \mathrm{~cm}^{-1}$. This is smaller than what is obse:ved, but considering the splitting between the ${ }^{3} \Pi_{u}$ $\left(0_{4} \pm\right)$ levels is not considered in the calculation, it seems quite close.

\section{v. Conclusion}

The work presented here again shows how the combination of anion PES and ZEKE spectroscopy provides a poweriul probe of electronically complicated species. The term energies and vibrational frequencies obtained for the five lowest-lying neutral triplet and singlet states of $\mathrm{Ge}_{2}$ were found to be consistent with ab initio values. Moreover, with Wellner's torm energy for the ${ }^{3} \Pi_{u}\left(1_{u}\right)$ state, we were able to determine the energy splitting between the ${ }^{2} \Pi_{u}$ (3/2) and ${ }^{2} \Sigma_{g}+$ states of the anion with less uncertainty than would have been afforded by the PES and ZEKE spectrum alone.

From the triplet band of the ZEKE spectrum, the zero field splitting between the $\mathrm{X}_{\mathrm{g}}{ }^{+}$ and $1_{\mathrm{g}}$ components of the ${ }^{3} \Sigma_{\mathrm{g}}{ }^{-}$neutral state was determined to be $114 \pm 5 \mathrm{~cm}^{-1}$, both components having a $286 \pm 5 \mathrm{~cm}^{-1}$ vibrational frequency. The term energies of the $2_{u,} 1_{u}, 0_{u}$ * and $\mathrm{O}_{u}{ }^{+}$components of the ${ }^{3} \Pi_{u}$ state, with their $308 \mathrm{~cm}^{-1}$ vibrational frequency (per Weltner ${ }^{10}$ ) were found to be $337,711,1193$, and $1305 \pm 14 \mathrm{~cm}^{-1}$, respectively. These energies are taken with respect to the zero-point energies. It was also determined that the ${ }^{2} \Sigma_{g}+\left\{\omega_{e}=326 \pm 10\right.$ $\left.\mathrm{cm}^{-1}\right)$ anion state lies $279 \pm 10 \mathrm{~cm}^{-1}$ above the ${ }^{2} \Pi_{\mathrm{y}}(3 / 2)\left(\omega_{e}=309 \pm 5 \mathrm{~cm}^{-1}\right)$ level.

From the singlet band, term energies for the ${ }^{1} \Delta_{g r}(1)^{1} \Sigma_{g}{ }^{+}$, and ${ }^{1} \Pi_{u}$ states were determined. The ${ }^{1} \Delta_{\mathrm{g}}\left(\omega_{\mathrm{e}}=276 \pm 3 \mathrm{~cm}^{-1}\right)$, as predicted, was found to be the lowest-lying with $T_{e}$ 
$=3308 \pm 7 \mathrm{~cm}^{-1}$. The $(1)^{1} \Sigma_{\mathrm{g}}{ }^{+}\left(\omega_{\mathrm{e}}=204 \pm 7 \mathrm{~cm}^{-1}\right)$ and ${ }^{1} \Pi_{\mathrm{u}}\left(\omega_{\mathrm{e}}=304 \pm 10 \mathrm{~cm}^{-1}\right)$ states are nearly degenerate, with $T_{e}$ 's around $4942 \pm 7 \mathrm{~cm}^{-1}$.

\section{Acknowledgments}

This work has been supported under NSF grant n. DMR-9201159. 
1 T. N. Kitsopoulos, C. J. Chick. Y. Zhao, and D. M. Neumark, J. Chem. Phys. 95, 1441 (1991); C. C. Amold, T. N. Kitsopoulos, and D. M. Neumark, J. Chem. Phys. 99, 766 (1993).

2 P. J. Bruna, S. D. Peyerimhoff, and A. J. Buenker, J. Chem. Phys. 72,5437 (1980); A. D. McLean, B. Liu, and G. S. Chandler, J. Chem. Phys. 80, 5130 (\$984); K. Raghavachari, J. Chem. Phys. 84, 5672 (1986); C. W. Bauschlicher, Jr. ano S. R. Langhofi, J. Chem. Phys. 87, 2919 (1987); H. P. Lüthi and A. D. McLean, Chem. Phys. Lett. 135, 352 (1987); F. Müller-Plathe and L. Laaksonen, Chem. Phys. Lett. 160, 175 (1989); K. Raghavachari and C. M. Rohlfing. J. Chem. Phys. 94, 3670 (199i).

${ }^{3}$ P. Ho and W. G. Breiland, App. Phys. Lett. 44, 51 (1984).

4 For example, J. Ho, M. L. Pollak, K. M. Ervin, and W. C. Lineberger, J. Chem. Phys. 99, 8542; J. G. Eaton, H. W. Sarkas, S. T. Amold, K. M. McHugh, and K. H. Bowen, Chem. Phys. Lett. 193, 141 (1992); G. F. Ganteior, D. M. Cox, and A. Kaidor, J. Chem. Phys. 94, 854 (1991).

5 D. W. Amold, S. E. Bradforth, T. N. Kitsopoulos, and U. M. Neumark, J. Chem. Phys. 95, 3753 (1991); T. N. Kitsopoulos, C. J. Chick, Y. Zhao, and D. M. Neumark, J. Chem. Phys. 95; 5479 (1991); C. C. Amold, Y. Zhao, 7. N. Kitsopoulos, and D. M. Neumark, J. Chem. Phys. 97, 6121 (1992).

6 K. D. Kolenbrander and M. L. Mandich, J. Chem. Phys. 92, 4759 (1990); Phys. Rev. Lett. 65, 2169 (1990); K. -D. Rinnen, K.D. Kolenbrander, A. M. DeSantolo, and M. L. Mandich, J. Chem. Phys. 96, 4088 (1992).

${ }^{7}$ C. Xu, E. de Beer, D. W. Amold, C. C. Arnold, and D. M. Neumark, J. Chem. Phys. 101, (1994, in press); C. C. Amold and D. M. Neumark, Can. J. Phys. (1994, in press).

${ }^{8}$ T. N. Kitsopoulos, C. J. Chick, A. Weaver, and D. M. Neumark, J. Chem. Phys. 93, 6108 (1990); C. C. Annold and D. M. Neumark, J. Chem. Phys. 99, 3353 (1993); C. C. Arnold and D. M. Neumark, J. Chem. Phys. 100; 1797 (1994). 
${ }^{9}$ G. Burton, C. Xu, C. C. Amold, and D.M. Neumark (in preparation).

${ }^{10} \mathrm{~S}$. Li, R. J. Van Zee, and W. Weltnel Jr., J. Chem. Phys. 100, 7079 (1994).

${ }^{11}$ K. Balasubramanian, J. Mol. Spectrusc. 123, 228 (1987); Chem. Rev. 90, 93 (1990).

12 P. J. Bruna and F. Grein, Mol. Phys. 74, 1133 (1991).

${ }^{13}$ G. Pacchioni, Mol. Phys, 49, 727 (1983); Chem. Phys. Lett. 107, 70 (1984).

14 J. E. Kingcade, H. M. Nagarathna-Naik, I. Shim, and K. A. Gingerich, J. Phys. Chem. 90. 2830 (1986).

$15 \mathrm{G}$. Herzberg, Molecular Spectra and Molecular Structure Vol. I- Spectra of Diatomic Molecules 2nd. Ed., Krieger Publishi... Company, Malabar, Florida (1989) p. 338.

${ }^{16} \mathrm{H}$. Lefebure-Brion and R. W. Field, Perturbations in the Spectra of Diatomic Molecules, Academic Press, Orlando (1986).

17 F. W. Froben and W. Schelze, Surf. Sci. 156, 765 (1985).

${ }^{16}$ E. P. Wigner, Phys. Rev. 73, 1002 (1948).

${ }^{19}$ S. Geltman, Phys. Rev. 112, 176 (1958); L. M. Branscomb, D. S. Burch, S. J. Smith, and S. Geltman, Phys. Rev. 111, 504 (1958).

${ }^{20}$ T. G. Kietz, M. A. Duncan, D. E. Powers, and R. E. Smalley, J. Chem. Phys. 74, 6511 (1981).

21 D. Proch and T. Trickl, Rev. Sci. Instrum. 60, 713 (1989).

22 J. M. Bakker, J. Phys. E 6, 785 (1973); 7, 364 (1974). 
25 K. Müller-Dethlefs, M. Sander, and E. W. Schlag, Z. Naturforsch 39a, 1089 (1984); Chem. Phys. Lett. 12, 291 (1984); K. Müller-Dethlefs and E. W. Schlag, Annu. Rev. Phys. Chem. 42, 109 (1991).

${ }^{24}$ R. J. Van Zee, R. F. Ferrante, and W. Weltner, Jr., J. Am. Chem. Sos. 83, 6181 (1985).

25 J. B. Tatum and J. K. G. Watson, Can. J. Phys. 49, 2693 (1971). 
Table (8.1) Peak positions, relative energies and assignments in both the PES and ZEKE spectra. The asterisk (") indicates that the peak was observed only in the PES. In the case where peaks are observed in both spectra, the detachment energy abtained from the ZEKE spectrum is used. It should be noted that $2_{u}, 1_{u}, 0_{u}{ }^{-}$and $0_{u}{ }^{+}$refer to the $\Omega$ components of the ${ }^{3} \Pi_{u}$ neutral state, and $\mathrm{O}_{g}{ }^{+}$and $1_{g}$ are the components of the ${ }^{3} \Sigma_{g}$ neutral state.

\begin{tabular}{|c|c|c|c|}
\hline Peak & $\begin{array}{l}\text { Binding Energy } \\
\qquad\left(\mathrm{cm}^{-1}\right)\end{array}$ & $\begin{array}{l}\text { Relative Energy } \\
\qquad\left(\mathrm{cm}^{-1}\right)\end{array}$ & $\begin{array}{c}\text { Assignment } \\
\text { neutral }+e \cdot(e K E=0) \leftarrow \text { anion }\end{array}$ \\
\hline$a_{0.0}$ & 16409 & -318 & $o_{\mathrm{c}}+\left(v^{\prime}=0\right) \leftarrow{ }^{2} \Pi_{u .3 / 2}\left(v^{\prime \prime}=1\right)$ \\
\hline$b_{2.0}$ & 16451 & -268 & $2_{u}\left(v^{\prime}=0\right) \leftarrow 2 \Sigma_{0}+\left(v^{\prime \prime}=1\right)$ \\
\hline$a_{1.0}$ & 16534 & -193 & $1_{a}\left(v^{\prime}=0\right) \leftarrow^{2} \Pi_{u .3 / 2}\left(v^{\prime \prime}=1\right)$ \\
\hline$A_{0.0}$ & 16727 & 0 & $0_{0}+\left(v^{\prime}=0\right) \leftarrow{ }^{2} \Pi_{u \cdot 3 / 2}\left(v^{\prime \prime}=0\right)$ \\
\hline $\mathrm{B}_{2.0}$ & 16785 & 58 & $2_{u}\left(v^{\prime}=0\right) \leftarrow 2 \Sigma_{a}+\left(v^{\prime}=0\right)$ \\
\hline$A_{1.0}$ & 16840 & 113 & $1_{0}(v=0) \leftarrow 2 \Pi_{u-3 / 2}\left(v^{\prime \prime}=0\right)$ \\
\hline${ }^{*} \mathrm{C}_{2 \mathrm{u}}$ & & -280 & $2_{u}\left(v^{\prime}=0\right) \leftarrow{ }^{2} \Pi_{u .3 / 2}\left(v^{\prime \prime}=0\right)$ \\
\hline$A_{0.9}$ & 17009 & 282 & $o_{a}+\left(v^{\prime}=1\right) \leftarrow{ }^{2} \Pi_{u \cdot 3 / 2}\left(v^{\prime \prime}=0\right)$ \\
\hline $8_{2.1}$ & 17103 & 376 & $\Sigma_{u}\left(v^{\prime}=1\right) \leftarrow 2 \Sigma_{a}+\left(v^{\prime \prime}=0\right)$ \\
\hline$A_{1.1}$ & 17129 & 402 & $1_{0}\left(v^{\prime}=1\right) \leftarrow{ }^{2} \Pi_{u .3 / 2}\left(v^{\prime \prime}=0\right)$ \\
\hline$B_{1.0}$ & 17159 & 432 & $1_{u}\left(v^{\prime}=0\right) \leftarrow 2 \Sigma_{\alpha}+\left(v^{\prime}=0\right)$ \\
\hline$A_{0.2}$ & 17300 & 573 & $0_{0}+\left(v^{\prime}=2\right) \leftarrow 2 \Pi_{u \cdot 3 / 2}\left(v^{\prime \prime}=0\right)$ \\
\hline $\mathrm{C}_{1 \mathrm{u}}$ & & -670 & $1_{u}\left(v^{\prime}=0\right) \leftarrow{ }^{2} \Pi_{u .3 / 2}\left(v^{\prime \prime}=0\right)$ \\
\hline$A_{1,2}$ & 17417 & 690 & $1_{0}\left(v^{\prime}=2\right) \leftarrow 2 \Pi_{u, 3 / 2}\left(v^{\prime \prime}=0\right)$ \\
\hline $\mathrm{B}_{1.1}$ & 17467 & 740 & $1_{u}\left(v^{\prime}=1\right) \leftarrow 2 \Sigma_{g}+\left(v^{\prime \prime}=0\right)$ \\
\hline$A_{0.3}$ & 17580 & 853 & $0_{a}+\left(v^{\prime}=3\right) \leftarrow{ }^{2} \Pi_{u .3 / 2}\left(v^{\prime \prime}=0\right)$ \\
\hline $\mathrm{B}_{0-0}$ & 17641 & 914 & $0_{u}\left(v^{\prime}=0\right) \leftarrow 2 \Sigma_{0}+\left(v^{\prime}=0\right)$ \\
\hline $\mathrm{B}_{0+, 0}$ & 17753 & 1026 & $o_{u}+\left(v^{\prime}=0\right) \leftarrow 2 \Sigma_{a}+\left(v^{\prime \prime}=0\right)$ \\
\hline${ }^{*} \mathrm{C}_{\mathrm{Ou}}$ & & -1200 & $o_{u}^{-}\left(v^{\prime}=0\right) \leftarrow{ }^{2} \Pi_{u .3 / 2}\left(v^{\prime \prime}=0\right)$ \\
\hline$B_{0-1}$ & 17966 & 1239 & $o_{u}\left(v^{\prime}=1\right) \leftarrow 2 \Sigma_{a}+\left(V^{\prime}=0\right)$ \\
\hline $\mathrm{B}_{0+.1}$ & 18067 & 1340 & $o_{u}+\left(v^{\prime}=1\right) \leftarrow 2 \Sigma_{0}+\left(v^{\prime \prime}=0\right)$ \\
\hline \multirow[t]{2}{*}{$d_{0}$} & 19734 & 3007 & ${ }^{1} \Delta_{a}\left(v^{\prime}=0\right) \leftarrow{ }^{2} \Pi_{u, 3 / 2}\left(v^{\prime}=1\right)$ \\
\hline & 19861 & 3134 & ? \\
\hline$D_{0}$ & $20035 / 20046$ & $3308 / 3319$ & ${ }^{1} \Delta_{0}\left(v^{\prime}=0\right) \leftarrow{ }^{2} \Pi_{u .3 / 2}\left(v^{\prime}=0\right)$ \\
\hline$D_{1}$ & 20312 & 3585 & ${ }^{1} \Delta_{a}\left(v^{\prime}=1\right) \leftarrow{ }^{2} \Pi_{u .3 / 2}\left(v^{\prime}=0\right)$ \\
\hline$D_{2}$ & 20586 & 3859 & ${ }^{1} \Delta_{a}\left(v^{\prime}=2\right) \leftarrow{ }^{2} \Pi_{u, 3 / 2}\left(v^{\prime \prime}=0\right)$ \\
\hline$D_{3}$ & 20846 & 4119 & ${ }^{1} \Delta_{a}\left(v^{\prime}=3\right) \leftarrow{ }^{2} \Pi_{u .3 / 2}\left(v^{\prime \prime}=0\right)$ \\
\hline$e_{0}$ & 21062 & 4335 & ${ }^{1} \Pi_{u}\left(v^{\prime}=0\right) \leftarrow{ }^{2} \Sigma_{0}+\left(v^{\prime \prime}=1\right)$ \\
\hline$D_{4}$ & 21119 & 4392 & ${ }^{1} \Delta_{a}\left(v^{\prime}=4\right) \leftarrow{ }^{2} \Pi_{u, 32}\left(v^{\prime}=0\right)$ \\
\hline$E_{0}$ & 21390 & 4664 & ${ }^{1} \Pi_{u}\left(v^{\prime}=0\right) \leftarrow 2 \Sigma_{a}+\left(v^{\prime \prime}=0\right)$ \\
\hline
\end{tabular}




\begin{tabular}{|c|c|c|c|}
\hline $\mathbf{g}_{1}$ & 21552 & 4825 & (1) ${ }^{1} \Sigma_{0}+\left(v^{\prime}=1\right) \leftarrow{ }^{2} \Pi_{u \cdot 3 / 2}\left(v^{\prime \prime}=1\right)$ \\
\hline$* F$ & & -4940 & ${ }^{1} \Pi_{u}\left(v^{\prime}=0\right) \leftarrow{ }^{2} \Pi_{u .3 / 2}\left(v^{\prime \prime}=0\right)$ \\
\hline $\mathbf{G}_{0}$ & $21668 / 21690$ & $4941 / 4963$ & $\begin{array}{c}\text { (1) }{ }^{1} \Sigma_{0}+\left(v^{\prime}=0\right) \leftarrow{ }^{2} \Pi_{u .3 / 2}\left(v^{\prime \prime}=0\right) ; \\
1 \Pi_{u}\left(v^{\prime}=1\right) \leftarrow{ }^{2} \Sigma_{q_{0}}+\left(v^{\prime \prime}=0\right)\end{array}$ \\
\hline$g_{2}$ & 21784 & 5057 & (1) ${ }^{1} \Sigma_{a}+\left(v^{\prime}=2\right) \leftarrow{ }^{2} \Pi_{u .3 / 2}\left(v^{\prime \prime}=1\right)$ \\
\hline$G$ & $2+872$ & 5145 & $(1)^{1} \Sigma_{\alpha}+\left(r^{\prime}=1\right)+{ }^{2} \Pi_{u .3 / 2}\left(v^{\prime \prime}=0\right)$ \\
\hline$E_{2}$ & 21997 & 5271 & ${ }^{1} \Pi_{u}\left(v^{\prime}=2\right) \leftarrow{ }^{2} \Sigma_{0}+\left(v^{\prime \prime}=0\right)$ \\
\hline
\end{tabular}


Table (B.2) Spectroscopic constants of the low-lying electronic states of $\mathrm{Ge}_{2}$ and $\mathrm{Ge}_{2}$-:

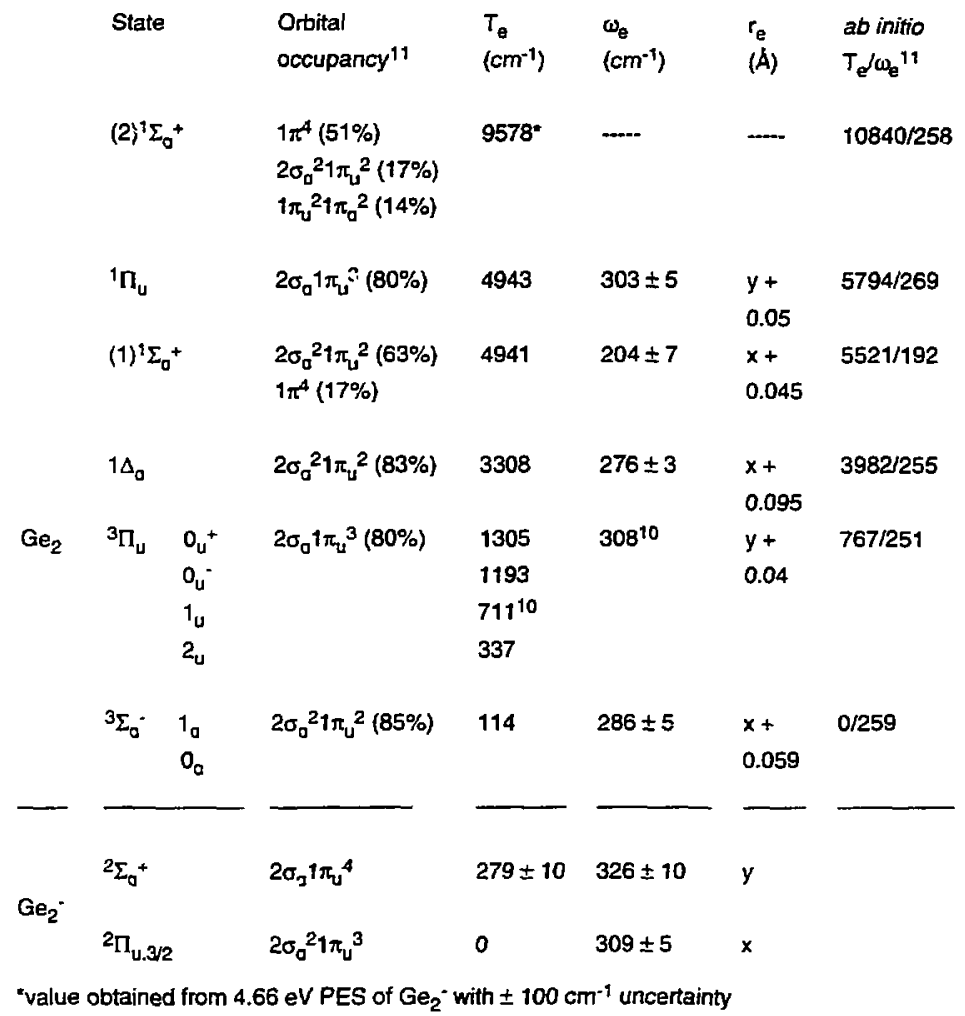


Figure (8.1) One-electron allowed transitions between the two anion and five neutral states discussed in the text. S-wave transitions are indicated by solid linfs, and p-wave by dashed lines. The left-most transition indicated by (") is that observed by Weltner [Ref. (10)] in matrix absorption studies.

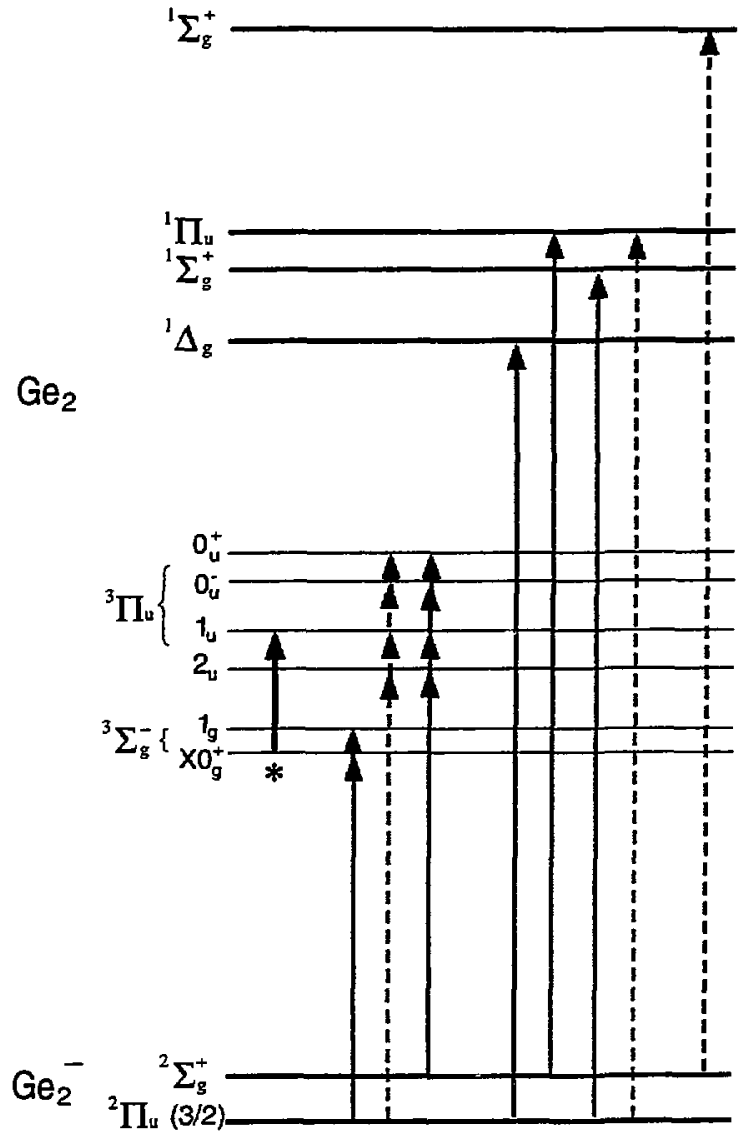


Figure (8.2) Photoelectron spectra of $\mathrm{Ge}_{2}{ }^{-}$obtained using $2.98 \mathrm{eV}$ photon energy at two different laser polarizations (dotted lines) and the ZEKE spectrum of $\mathrm{Ge}_{2}^{-}$(solid line).

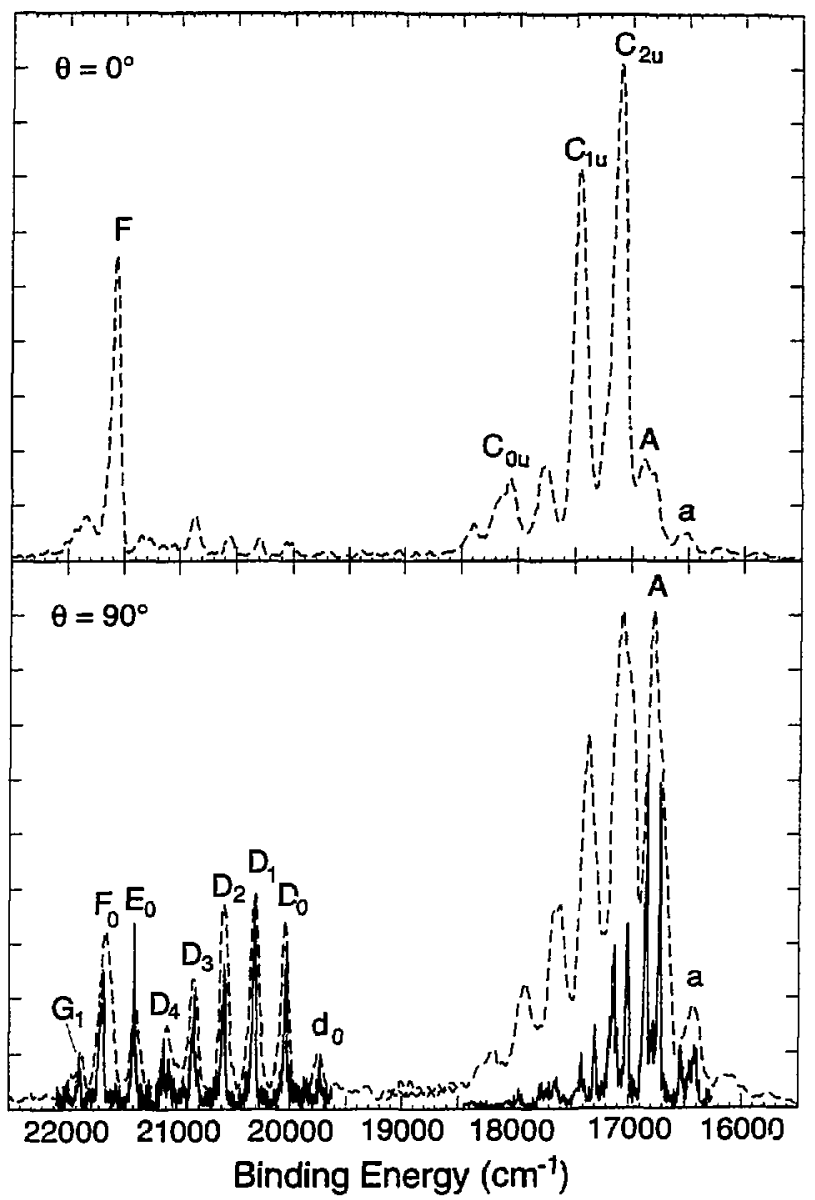


Figure (8.3) Triplet band of ZEKE spectrum of $\mathrm{Ge}_{2}-$ (solid line, upper trace) and simulations of the two electronic transitions responsible for structure (below; lighter line indicates the ${ }^{3} \Sigma_{g}{ }^{+}$ $\left(1_{g}, 0_{g}^{+}\right) \leftarrow 2 \Pi_{u}(3 / 2)$ transitions, heavier dashed line indicates the ${ }^{3} \Pi_{u}\left(0_{u} \pm, 1_{u}, 2_{u}\right) \leftarrow 2 \Sigma_{g}$ transitions).

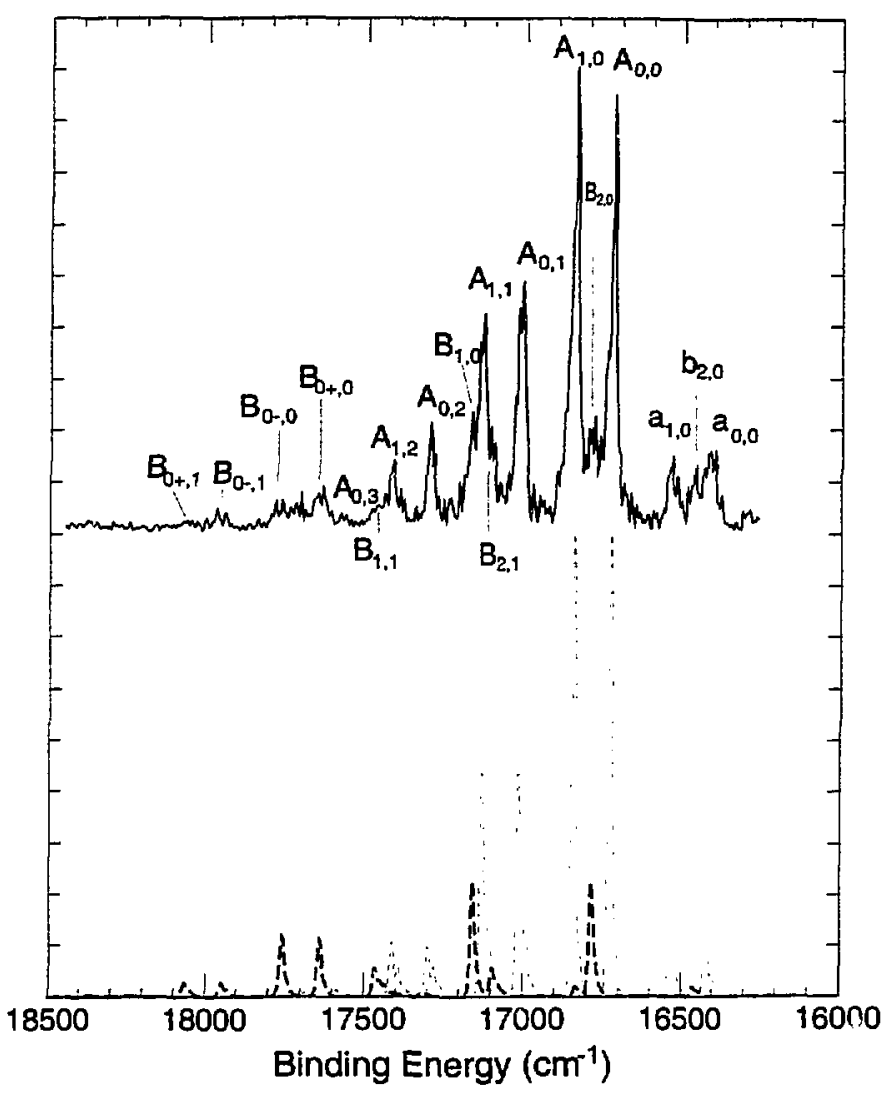


Figure (8.4) Singlet band of ZEKE spectrum of $\mathrm{Ge}_{2}^{-}$(solid line) and spectral simulations (dotted line, see text).

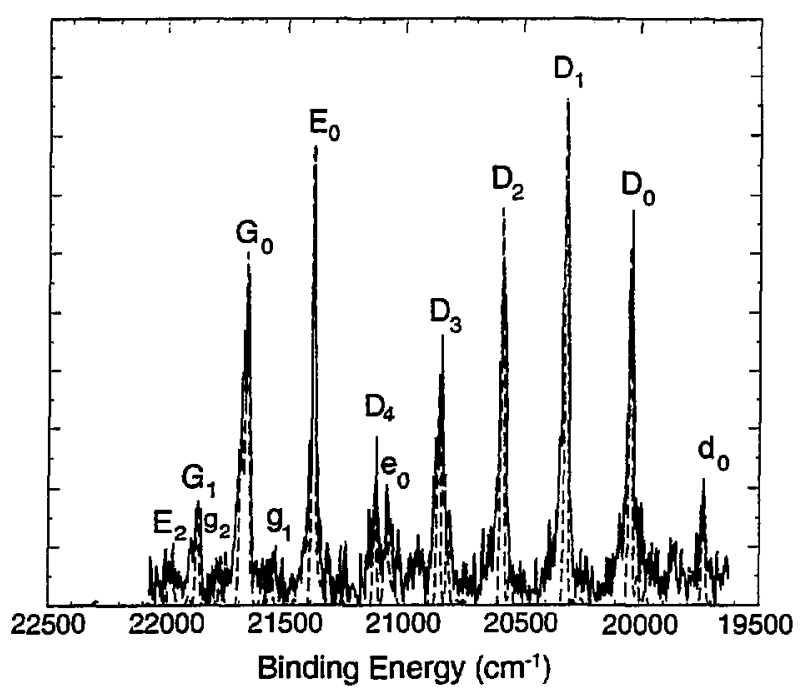




\section{Chapter 9. Study of $I n_{2} P / / n_{2} P-$ and $I n P_{2} / n P_{2}-$ using negative ion zero electron kinetic energy spectroscopy}

\section{Abstract}

The zero electron kinetic energy (ZEKE) spectra of $\ln _{2} \mathrm{P}^{-}$and $\ln _{2}{ }^{-}$are presented and compared to their previously obtained photoelectron spectra (PES) as well as ab initio calculations on analogous species. The threshold spectra, which give high accuracy electron affinities of $2.400 \pm 0.001 \mathrm{eV}$ for $\ln _{2} P$ and $1.617 \pm 0.001 \mathrm{eV}$ for $\ln _{2}$, show well-resolved vibrational structure in the transitions from the ground anion states to the various neutral states. The ZEKE spectrum of $I n_{2} P^{-}$exhibits a failly extended, $47 \mathrm{~cm}^{-1}$ progression that we assign to the symmetric bend $\left(v_{2}\right)$ in the ground ${ }^{2} B_{1}$ neutral state. There is also a $204 \mathrm{~cm}^{-1}$ progression that we assign to the symmetric stretch. The $\mathrm{InP}_{2}{ }^{-}$ZEKE spectrum shows transitions to two electronic states of the neutral. For the ground state, the symmetric stretch mode is the most active in the spectrum, while in the excited state, the symmetric bend mode is most active. The $\ln P_{2}$ ground state symmetric stretch frequency is $190 \mathrm{~cm}^{-1}$, and the excited state symmetric bend frequency is $287 \mathrm{~cm}^{-1}$. An anion ground state frequency is determined to be $227 \mathrm{~cm}^{-1}$. The term energy of the excited state is determined to be $1.280 \pm 0.001 \mathrm{eV}$. Based on molecular orbital arguments, these frequencies suggest a ${ }^{2} B_{2}$ ground $\ln P_{2}$ state, $a{ }^{2} A_{1}$ first excited state, and $a^{1} A_{1}$ anion ground state. 


\section{Introduction}

A considerable experimental and theoretical effort has been focused on the study of very small clusters in the hope that, by understanding the bonding in these species, insight toward the evolution of matter between the atomic and bulk scales will be gained. Clusters of pure semiconductor materials such as silicon have been of particular interest, owing to their potential technological importance. Mixed semiconductors are also important materials in circuit components as well as more exotic devices such as infrared light detectors and semironductor injection lasers. ${ }^{1}$ On the whole, spectroscopic investigations of pure semiconductcr clusters have tended to lag behind the calculations, due to the dual requirements of size-selectivity and spectral resolution in cluster spectroscopy experiments. For instance, only recently have ground and excited state frequencies been determined for $\mathrm{Si}_{n}(n=3,4,6,7), 2,3,4,5$ while calculations have been ongoing for almost ten years. 6

Mixed semiconductor clusters present the same experimental challenges, arid are also more of a challenge from a theoretical standpoint because of the wider range of structural permutations available in a binary system. Several calculations on small GaAs, InSb, and AIP clusters $7,8,9,10,11$ have determined that multiple structural pemutations possible for these species can in fact be close-lying in energy. In addition, each close-lying permutation may be congested with close-lying electronic states due to dangling bonds and, in some cases, open shells. The molecular geometries of small clusters are very different from bulk structures. Some of the compelling experimental questions regarding these small species, therefore, revolve around how the bonding in small clusters compare between clusters of similar atoms (i.e. the various III-V semiconductors) and how the bonding changes in clusters of different stoichiometries. The experimental work so far on mixed clusters leaves these questions largely unanswered. For example, while the electronic spectroscopy of several small GaAs and InP 
clusters has been studied by photoelectron and photodissociation spectroscopy, vibrationaf frequencies have only been measured for the diatomic and some of the triatomic species. ${ }^{12}$

In this paper, we present zero electron kinetic energy (ZEKE) studies on $\ln _{2} P^{-}$and $\ln P_{2}-$ which, along with our previously obtained negative ion photoelectron spectra (PES), ${ }^{13}$ were in part motivated by the experimental work on small indium phosphide clusters done by Mandich and coworkers. By using resonant one-color and two-color photodissociation spectroscopy, Mandich mapped out the absorption of several clusters between 0.84 and $1.84 \mathrm{eV} .^{14}$ One of the interesting findings of Mandich's studies was that the energy of the HOMU-LUMO gap (or, more accurately, the lowest-energy transition allowed from the ground state) for the clusters, both stoichiometric and non-stoichiometric, was very near the band gap of bulk $\ln P$, although the oddnumbered clusters in her studies exhibited stronger absorption at lower energies. The PES studies of $\ln _{x} P_{y}-$ mentioned above were complementary to Mandich's experiments; the clusters we investigated were in general smaller than those studied by Mandich, and more of the neutral states were accessed in our studies, but we observed size-dependent electronic structural trends similar to those revealed by Mandich. The only other work to date on small InP clusters is the infrared matrix isolation study by Weltner which yielded the InP diatomic frequency and the $v_{3}$ frequency for $\ln _{2} P .12$

Both the work of Mandich and our PES data yielded some electronic structure for several mass-determined InP clusters, but no vibrational structure was resolved. The types of infrared or electronic absomtion experiments that could determine vibrational frequencies are in general complicated by the presence of multiple species generated by most cluster sources. On the other harid, negative ion ZEKE spectroscopy is particularly well suited toward small cluster study, as it combines both mass selection and spectral resolution as high as $2-3 \mathrm{~cm}^{-1}$ (compared to 60-100 $\mathrm{cm}^{-1}$ for anion PES). Thus far, we have applied this technique toward the investigation of $\mathrm{C}_{5}^{-},{ }^{-15} \mathrm{C}_{6}{ }^{-16}$ and $\mathrm{Si}_{n}^{-}(\mathrm{n}=2,3 \text {, and } 4)^{4,5}$ and have been able to resolve the lowfrequency modes and closely-spaced sequence band structure characteristic of these species. 
The negative ion ZEKE spectra of $\ln _{2} P^{-}$and $\ln _{2}-$ presented below show rich electronic and vibrational structure that was unresolved in the PES, yielding detailed information on both the anion and neutral species. The $\ln _{2} P$ - ZEKE spectrum shows transitions to vibrational levels in at least two modes of the ground state of the neutral. The $\ln \mathrm{P}_{2}^{-}$spectrum exhibits transitions to two electronic states of the neutral: the ground state and a low-lying excited state. The ZEKE spectra are compared with the corresponding $\mathrm{PES}_{1}{ }^{13}$ as well with the calculations on the analogous $\ln _{x} \mathrm{Sb}_{y}$ and $\mathrm{Ga}_{x} \mathrm{As}_{\mathrm{y}}$ triatomics by Balasubramanian ${ }^{7}$ and the $\mathrm{GaAs}_{2} / \mathrm{GaAs}_{2}{ }^{-}$anion calculations by Meier. ${ }^{9}$ To aid in our analysis of $\ln P_{2}$, we have also performed our own ab initio calculations for trequencies and geometries at the MP2 level using an effective core potential basis set. The interesting frequency trends predicted for the two lowest-lying states of in $P_{2}$ are bom out well in our spectra. While we have not performed any frequency calculations for $\ln _{2} P$. the vibrational structure observed in the $\ln _{2} P^{-}$spectrum is consistent with he calculated ground electronic states of $\mathrm{Ga}_{2} \mathrm{As}$ and $\mathrm{In_{2 }} \mathbf{S b}$.

The ZEKE spectra strongly indicate that the anions and neutrals for both species have $C_{2 v}$ geometries. In the $\ln _{2} P$ neutral ground state, the bonding between the phosphorous atom and the indium atoms is much stronger than the bonding between the indium atoms, which is at most very weak. The anion exhibits stronger bonding among all of the atoms. For the $\ln \mathrm{P}_{2}$ neutral ground state, the bonding between the two phosphorous atoms appears to be a strong. double covalent bond, with single bonds connecting each phosphorous to the indium atom. This is in contrast to th: "It excited state, which has comparatively weakened P-P bonding and stronger In-P bonding. The bonding properties in the $\mathrm{InP}_{2}^{-}$ground state appear to lie between the two neutral states. These observations can be explained with reference to the molecular ortitals for the valence electrons. 


\section{Experiment}

The threshold photodetachment apparatus used in these studies has been discussed in detaii elsewhere, ${ }^{17}$ but will be described briefly here. Figure (9.1) shows a schematic diagram of the apparatus employed in these experiments. A beam of cold indium phosphide cluster anions is generated in a laser vaporization/pulsed-molecular beam source similar to that developed by Smalley and coworkers. ${ }^{18}$ The surface of a rotating, translating rod of InP is vaporized using 2 to $4 \mathrm{~mJ} / \mathrm{pulse}$ of the 2 nd harmonic output of a Nd:YAG operated at $20 \mathrm{~Hz}$ repetition rate. The resulting plasma is entrained in a pulse of He carrier gas from the molecular beam into a clustering channel. The gas then expands into the source chamber, and those negative ions passing through a $2 \mathrm{~mm}$ skimmer are colinearly accelerated to $1 \mathrm{keV}$ and mass-selected using time-of-flight. The cluster of interest is then selectively photodetached using a (pulsed) excimerpumped tunable dye laser. Only the electrons detached with nearly zero kinetic energy in the anion beam frame are collected as a function of photon energy. This selective detection of threshoid electrons, based on the techniques developed by Müller-Dethlefs et al. for photoionization of neutrals, ${ }^{19}$ gives an energy resolution of $3 \mathrm{~cm}^{-1}$.

For both molecules, the spectra were first obtained using the cluster source configuration that produced the coldest $\mathrm{Si}_{4}^{-}$spectrum; ${ }^{4}$ a piezoelectric molecular beam valve used in conjunction with a $0.2^{\prime \prime}$ long, $0.15^{\prime \prime}$ inner diameter clustering channel terminated with a $0.1 "$ exit slit. This generates clusters with a vibrational temperasture of approximately $200 \mathrm{~K}$ (higher frequency modes generaliy are hotter, while lower frequency modes are usually more efficiently cooled). After obtaining spectra of ions generated with the piezoelectric valve source, portions of the spectra werte retaken using a new source incorporating a liquid nitrogen-cooled

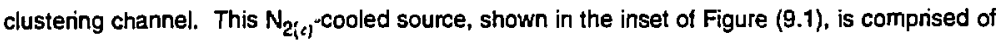


a Vespel piece attached to the pulsed valve, and the copper clustering channel. Laser vaporization and molecular beam entrainment occurs in the Vespel piece, which serves to thermally insulate the pulsed valve from the cold clustering channel. The slustering channel is $1.25^{\prime \prime}$ long with an inner bore of $0.10^{\prime \prime}$. A terminal exit slit on the clustering channel was also required for the most effective cooling in this source configuration; it is also made of copper, and rias an inner diameter of $0.05^{\circ}$. A $1 / 8^{\prime \prime}$ copper tube camying liquid nitrogen is fastened directly to the outside of the channel. Even with the insulating Vespel piece, some cooling of the pulsed vaive occurred. We found the General Valve solenoid-type molecular beam valve to be more reliable with this sruurce configuration as the valve plunger tip was less sensitive to temperature than the $\mathrm{O}$-ring tip used in the piezoelectric valve. By retaking a portion of the $\mathrm{Si}_{4}{ }^{-}$spectrum, we determined that the use of the cooled clustering channel further enhanced cluster cooling to a vibrational temperature of approximately $100-130 \mathrm{~K}$.

For $1 n_{2} P^{-}$, the photodetachment cross section was unusually large, and the signal was averaged for only 800 shots/point for the $200 \mathrm{~K}$ spectrum and 600 shots/point for the $N_{2(t)}$ portion of the spectrum. The dyes used for the spectrum were Coulnarin 503, Coumarin 480 , and Coumarin 450 . For $\operatorname{In}_{?}^{2}$, the spectrum was signal averaged for 1500 shots/point for the $200 \mathrm{~K}$ spectrum, and 800 shots/point for the $\mathrm{N}_{2(\iota)}$ portion. The dyes used for $\ln \mathrm{P}_{2}{ }^{-}$were Rhodamine 800, LDS 722, LDS 698, Nile Blue 690, DCM, Rhodamine 640, Coumarin 440 , Exalite 416, DPS and QUI.

\section{Results}

\section{A. $\ln _{2} P-$}

Figure (9.2) shows the ZEKE spectrum of $\mid n_{2} p-$ (solid line) plotted vs. detachment wavelength. This is superimposed onto the previously obtained PES obtained with $4.66 \mathrm{eV}$ 
photon energy (dotted line). ${ }^{13}$ For the energy scale of the PES, shown on the upper $x$ - axis, the electron kinetic energy (eKE) is given by

$$
\text { eKE }=h v-E A-E_{\text {int }} \text {, }
$$

where hv is the photon energy $(4.66 \mathrm{eV}), \mathrm{EA}$ is the electron affinity of the neutral cluster, and $E_{\text {int }}$ is the interraal energy (vibrational, electronic, etc.) of the final neutral state. The PES shows two bands labeled $X$ and $A$, which are 0.1 to $0.15 \mathrm{eV}$ wide, respectively, and spaced by $0.23 \mathrm{eV}$. These correspond to transitions to two electronic states of the $\ln _{2} P$ : the ground state $(X)$ and a low-lying state (A). The ZEKE spectrum in the energy region of band $\mathbf{X}$ shows extensive vibrational structure spaced by $47 \mathrm{~cm}^{-1}$ which was unresolved in the PES. It is also apparent from the superimposed spectra that band $\mathbf{A}$ in the PES is absent in the ZEKE spectrum.

A detailed look at the ZEKE spectrum of band $\mathbf{X}$ is shown in Figure (9.3), in which the upper trace was obtained from $\ln _{2} P^{-}$generated using the $N_{2(\iota)}$-cooled channel in ciuster production, and the center trace was obtained using the room temperature source. The peaks in both spectra are $8 \mathrm{~cm}^{-1}$ full width, half maximum (FWHM). The signal to noise is clearly better in the upper trace, the baseline is better-resolved between 510 and $515 \mathrm{~nm}$, and several of the sinaller peaks are less intense in the upper spectrum. All of these effects are consistent with colder ions in the upper spectrum and aid in identifying hot band transitions from v'trationally excited anions, such as the peak indicated with an asterisk (") which is more intense in the center spectrum than in the upper spectrum.

The spectra show several vibrational progressions indicated by the comos in Figure (9.3). The characteristic spacing in each progression is $47.0 \pm 0.3 \mathrm{~cm}^{-1}$. Peak $\mathrm{B}$ is the apparent origin of the most intense progression, labeled the $2_{0}^{v}$ progression, and is the lowest energy peak originating from the anion ground vibrational state. This yields an electron affinity of 2.400 $\pm 0.001 \mathrm{eV}$ tor $\ln , \mathrm{P}$. Three less intense progressions, labeled $2{ }_{0}^{y} 1_{0}^{n}(n=1-3)$, are found to the 
blue of the $2_{0}^{v}$ progression, and the origins of the four progressions are spaced by $204 \pm 2 \mathrm{~cm}^{-1}$. We are therefore observing vibrational progressions in two vibrational modes $\left(v_{1}\right.$ and $\left.v_{2}\right) c^{f}$ neutral $l n_{2} P$. The assignments of these modes will be discussed in detail in the next section, but for the purpose of presenting the data, the combs will serve as peak labels. There is also a peak found at $304 \mathrm{~cm}^{-1}$ from the most intense $2_{0}^{2}$ peak indicated with an arrow ( $\downarrow$ ). If is approximately at the right position to be the $2_{0}^{0} 1_{0}^{2}$ transition, but based on the patterns in the spectrum, it is anomalously intense. In the upper spectrum, there is a progression, labeled $2_{0}^{u} 3_{1}^{\text {? }}$ in which each peak is $27 \mathrm{~cm}^{-1}$ to the blue of a peak in the $2_{0}^{0}$ progression. The $2_{0}^{v} 3_{1}^{1}$ progression

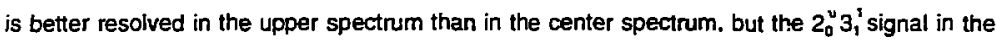
center spectrum overall seems to have more intensity. This structure appears to be a sequence band originating from vibrationally excited anions (with $v_{3}=1$ ) and will be discussed in detail below.

In the $\mathrm{N}_{2(\ell)}$-cooled configuration, we also scanned the laser to lower photon energy and found the low-intensity hot bands between 519 and $525 \mathrm{~nm}$ (approximately $300 \mathrm{~cm}^{-1}$ to the red of the $2_{0}^{u}$ progression). This group of peaks has a characteristic spacing of around one half the $47 \mathrm{~cm}^{-1}$ spacing of the $2_{0}^{\nu}$ progression. These features, along with the $2_{c}^{u} 3_{1}^{\prime}$ progression, appear to be the only progressions attributable to transitions from vibrationally excited anions.

Peak positions and relative energies for the ZEKE spectrum of $I_{2} P^{-}$are summarized in Tabie (9.1).

\section{B. In $P_{2}^{-}$}

The PES of $\operatorname{InP}_{2}-$ obtained using a photon energy of $4.66 \mathrm{eV}^{13}$ is shown in the bottom panel of Figure (9.4). As with $\ln _{2} P$, this spectrum shows transitions to two electronic states of the neutral, labeled bands $X$ and $A$. The two upper panels in Figure (9.4) show the ZEKE spectra of $\ln \mathrm{P}_{2}-$ plotted against detachment wavelength superimposed onto the vibrationally 
resolved PES obtained using two lower photon energies; the $355 \mathrm{~nm}$ spectrum of band A (the higher eKE region of the $355 \mathrm{~nm}$ spectrum was truncated to show only band A) shows a 280 $\mathrm{cm}^{-1}$ progression, and the $532 \mathrm{~nm}$ spectrum shows a partially resolved $190 \mathrm{~cm}^{-1}$ progression band $X$. More vibrational structure appears in the upper spectra because the resolution improves with lower eKE. In contrast to the $\operatorname{In}_{2} \mathrm{P}^{-}$spectrum, the two bands in the $\ln \mathrm{P}_{2}{ }^{*}$ spectrum are $0.3 \mathrm{eV}$ wide and spaced by approximately $1.28 \mathrm{eV}$. ZEKE spectra are observed for both bands, although in both cases, the intensity profile of the structure in the ZEKE spectra does not appear to match the profiles of the bands observed in the PES. More detailed views of the ZEKE spectra of band $X$ and band $A$ are found in Figures (9.5) and (9.6), respectively. Again, spectra obtained under the two source conditions are presented, with the "colder data presented in the upper panel, and the data acquired using the room temperature cluster source in the center panel.

\section{1) Band $x$}

The ZEKE spectrum of band $X$ shown in Figure (9.5) is dominated by a $190.0 \pm 0.2 \mathrm{~cm}^{-1}$ progression, with progression members indicated on the $2_{0}^{v}$ comb. Because of the extension of the progression, the origin transition will have very low intensity, and may in fact be obscured by the noise. The $2_{0}^{0}$ peak corresponds to the lowest energy peak observed in this progression. We therefore take it as the origin, yielding an electron affinity for $\ln P_{2}$ of $1.617 \pm 0.001 \mathrm{eV}$.

At higher photon energy, there is another, somewhat less intense progression with a characteristic spacing of $190 \mathrm{~cm}^{-3}$ indicated with the $12_{0}^{4}$ comb. In the center spectrum, the $1_{0} 2_{0}^{4}$ is the most intense member of the $1_{0}^{1} 2_{0}^{v}$ progression, peak $2_{0}^{4}$ is the most intense of the $2_{0}^{v}$ progression, and the two peaks are spaced by $479 \mathrm{~cm}^{-1}$, suggesting a $479 \mathrm{~cm}^{-1}$ frequency for a second neutral $\left(v_{1}\right)$ mode. This frequency is not entirely definitive from the spectrum, since the determination of the origin of the $1_{0}^{j} 2_{0}^{v}$ progression is made ambiguous by the sequence band progression, $2_{0}^{v} 3_{1}^{1}$, which overlaps somewhat with the $1_{0}^{1} 2_{0}^{v}$ progression. Each peak in the $2_{0}^{v} 3_{1}^{\prime}$ progression is approximately $78 \mathrm{~cm}^{-1}$ to the red of a 20 transition, while the $1_{0}^{1} 2_{0}^{\nu}$ transitions are 
approximately $92 \mathrm{~cm}^{-1}$ to the red of the $2_{0}^{p+3}$ transitions. While this difference in spacing aliows us to discem unambiguously between the two progressions near the origin (where there are no $1_{0} 2_{0}^{0}$ transitions) and at higher photon energies (where the $1_{0} 2_{0}^{\prime \prime}$ transitions will dominate) the first few members of the $1_{0}^{1} 2_{0}^{\nu}$ progression are small and overlap with the more intense part of the $2{ }_{0}^{u} 3{ }_{1}^{1}$ progression, giving rise to the possibility that the $v_{1}$ frequency is actually $(479+190) \mathrm{cm}^{-1}$ or $(479-190) \mathrm{cm}^{-1}$. However, the $479 \mathrm{~cm}^{-1}$ frequency is supported by our ab initio calculations presented below.

There is another progression, the $2_{1}^{v+1}$ progression, displaced $37 \mathrm{~cm}^{-1}$ to lower photon energy from the $2_{0}^{\nu}$ progression and assigned to a hot band progression originating from the anion $v_{2}=1$ level. The intensity profile of this progression differs somewhat from the others in that it peaks earlier. While the intensities of the $2_{1}^{v+1}$ progression are not unifomly less intense in the upper spectrum than in the center spectrum, the intensities in the center spectrum appear to be complicated by the overall congestion and lack if baseline between peaks. The $2{ }^{3}, 2$, and $2{ }_{1}^{5}$ transitions, however, are less intense in the upper spectrum. Another progression, labeled $1_{0}^{1} 2_{1}^{u+i}$ and displaced $37 \mathrm{~cm}^{-1}$ to the red of the $1_{0}^{t} 2_{0}^{0}$ progression, similarly peaks earlier than the $1,2_{0}^{y}$ progression.

Peak positions and relative energies for band $X$ are summarized in Table (9.2).

\section{2) Eand A}

Band $A$ shown in Figure (9.6) is dominated by a single progression with a $287 \pm 1 \mathrm{~cm}^{-1}$ characteristic spacing indicated by the $2_{0}^{0}$ comb. The origin of this progression appears to be at $428.09 \mathrm{~nm}$. The energy difference between the origins of band $X$ and band $A$ gives a term energy of $1.280 \pm 0.001 \mathrm{eV}$ for the excited state. While there appears to be only one active mode in the spectrum, there are two additional progressions displaced approximately 60 and 75 $\mathrm{GIn}^{-1}$ to the blue of the $2_{0}^{\mathrm{u}}$ progression; these are labeled the $2,{ }_{1}^{u+1}$ and $2_{0}^{\mathrm{u}} 3_{1}^{\prime}$ progressions respectively, and have been assigned to transitions from singly excited anions. There are also several lower-intensity peaks apparently due to transitions from doubly excited anion vibrational 
levels. The main $2_{0}^{v}$ progression in the ZEKE spectrum [Figure (9.6)] nol only fails to span the width of band $A$ in the PES [Figure (9.4)] but the progression members broaden noticeably for $v$ $>2$.

While the profile of the $2_{0}^{\mathrm{v}}$ progression is clearly perturbed, it can still be seen that the relative intensities of peaks in the hot band $2_{\uparrow}^{\nu+1}$ progression compared to the $2_{0}^{v}$ peaks depend on $v$; for $v=0$ and 1 , the hot band intensities are greater than the corresponding transitions from the cold ions, while for $v=2$, the hot band has less than half the intensity of the cold transition. In contrast, the intensity distribution in the $2_{0}{ }^{1} 3_{1}^{1}$ progression approximately follows that of the $2_{0}^{0}$ progression.

A summary of the peak positions and relative energies for band A can be found in Table (9.3).

\section{3) $A B$ initio results}

Previous $a b$ initio calculations ${ }^{7,9}$ on both $\mathrm{GaAs}_{2}$ and $\operatorname{lnSb}_{2}$ predict a ${ }^{2} \mathrm{~B}_{2}$ ground state and low-lying ${ }^{2} A_{1}$ excited state, and a ${ }^{1} A_{1}$ ground state for $\mathrm{GaAs}_{2}^{-}$(no calculations on InSb ${ }_{2}^{-}$ have yet been published). Using the Gaussian 92 software package, ${ }^{20}$ geometry and frequency calculations at the MP2 level using the LANLDZ (Los Alanios National Laboratory double zeta) effective core potential were performed for the two isovalent neutral states of $\ln P_{2}$ as well as the corresponding ${ }^{~} \mathrm{~A}_{1} \operatorname{In} \mathrm{P}_{2}{ }^{-}$anion state.

The geometries, frequencies, and normal coordinates for these states in $\ln P_{2}$ as determined by the MP2 calculation are shown in Figure (9.7). There are striking differences between the geometries and vibrational modes of the two neutral states. For instance, compared to the ground ${ }^{2} \mathrm{~B}_{2}$ neutral state, the excited ${ }^{2} \mathrm{~A}_{1}$ state has a shorter in-P bond by 0.34 $A$ and a $0.3 \hat{A}$ longer P.P bond. The dramatic differences between the vibrational frequencies are also reflected in the disparate normal coordinates, as shown in Figure (9.7). The term energy for the ${ }^{2} \mathrm{~A}_{1}$ neutral state was calculated to be $1.139 \mathrm{eV}$. The ${ }^{1} \mathrm{~A}_{1}$ anion ground state has 
both intermediate bonding relative to the two neutral states, and, except for $v_{1}$, intermediate vibrational frequencies.

While our calculations are not as sophisticated as those performed by Balasubramanian ${ }^{7}$ and Meier, ${ }^{9}$ we will see that the vibrational frequencies, geometry changes, and $\ln \mathrm{P}_{2}$ excited state energy are consistent with the two bands in the $\operatorname{In} \mathrm{P}_{2}-Z E K E$ spectrum. In assigning features in the ZEKE spectrum, it is therefore reasonable to assume that the $\operatorname{InP}_{2}$ ground and excited states and the $\ln _{2}$ ground state are indeed the ${ }^{2} \mathrm{~B}_{2},{ }^{2} \mathrm{~A}_{1}$, and ${ }^{1} \mathrm{~A}_{1}$ states, respectively, with properties close to those shown in Figure (9.7). This assignment will be considered in more detail in the Discussion section.

\section{Analysis}

In order to understand the vibrational structure in the ZEKE spectra, it is uselul to have a reasonable idea of the likely anion and neutran geometnies. Without any a priori knowledge about these molecules, we are unable determine the point group of either based on the spectra alone. For $\ln _{2} \mathrm{P}^{*} / / \mathrm{n}_{2} \mathrm{P}$ and $\ln \mathrm{P}_{2}{ }^{-} / \mathrm{n} \mathrm{P}_{2}$, there are four possible geometries that these species can assume: $C_{2 v}, D_{\infty h h}, C_{m v}$ and $C_{s}$. Balasubramanian's calculations on analogous neutrals ${ }^{7}$ suggested that the $C_{\infty \circ v}$ (i.e., Ga-As-As) and $c_{s}$ structures are unstable with respect to rearrangement to $C_{2 v}$ structures. Our $a b$ initio results on $\operatorname{In} P_{2}^{-}$and $\ln P_{2}$ states presented in the previous section are on $\mathrm{C}_{2 v}$ species, but as we made no attempt to establish the universal minimal anion and neutral geometries, we rely on the $a b$ initio calculations by Meier et al. ${ }^{9}$ who predicted that the linear structures for the analogous $\mathrm{GaAs}_{2}^{-}$and $\mathrm{GaAs}_{2}$ were much higher in energy than the bent structures. Very acute bond angles $\left(<50^{\circ}\right)$ are predicted for the ground states of $\mathrm{InSb}_{2}, \mathrm{GaAs}_{2}$ and $\mathrm{GaAs}_{2}{ }^{-}$, and the low-lying excited states of InSb $\mathrm{S}_{2}$ and $\mathrm{GaAs}_{2}$ are also predicted to be strongly bent. ${ }^{7}$ If the predicted trends hold for $\ln \mathrm{P}_{2}$, then one would expect 
the $\ln \mathrm{F}_{2}-\mathrm{ZEKE}$ spectrum to represent transitions between $\mathrm{C}_{2 v}$ anion and neutral structures we have calculated.

The $\mathrm{Ga}_{2} \mathrm{As}$ and $\mathrm{In}_{2} \mathrm{Sb}$ ground states are predicted to have an obtuse geometries with bond angles of $109.5^{\circ}$ and $104^{\circ}$, respectively, ${ }^{7}$ but no calculations on the anions analogous to $I n_{2} P^{-}$have been performed. However, the progressions in the ZEKE spectrum are relatively short, indicating a fairly small change in geometry between anion and neutral. Thus, if neutral $\ln _{2} P$ is an obtuse $C_{2 v}$ structure with a bond angle in the range of $100-110^{\circ}$, then the anion most likely has an obtuse $\mathrm{C}_{2 v}$ structure as well. On the other hand, the calculations predict low-lying linear $\left(D_{\infty h}\right)$ excited states for both $\mathrm{Ga}_{2} \mathrm{As}$ and $\mathrm{In}_{2} \mathrm{Sb}$, so we must consider the possibility that these are in fact the ground states and that we are observing transitions from linear or nearly linear $\ln _{2} P^{-}$and linear $\ln _{2} P$.

\section{A. $\ln _{2} P-\pi n_{2} P$}

\section{1) Band $X$ vibrational structure and peak assignments}

Given the above discussion, the geometries of both the neutral and anion can be inferred with reasonable certainty considering several points. First, the $\ln _{2} P^{-}$ZEKE spectrum exhibits a substantial amount of activation in two modes of the neutral, with the $47 \mathrm{~cm}^{-1}$ mode being more active than the $204 \mathrm{~cm}^{-1}$ mode. In electronic spectroscopy, one typically observes progressions only in totally symmetric vibrational modes. The presence of two active modes therefore argues against a linear $\leftrightarrow$ - linear transition, as this would show significant activation in the sole totally symmetric mode with possibly a small amount of activation in double quanta of the degenerate bend and/or the antisymmetric stretch. Moreover, if the neutral were linear, spin-orbit splitting effects would be obvious in the spectra since there is at least one unpaired electron in $\ln _{2} P$; no such effects are observed. From Balasubramanian's calculations, the lowest-lying linear state is a ${ }^{2} \Pi_{u}$ state, but even if it were a ${ }^{2} \Sigma$ state, the zero field splitting should be on the order of several hundred wave numbers (for $I_{2}$, the energy splitting between 
the components of the lowest-lying ${ }^{3} \Sigma$ state was calculated to be on the order of $0.1 \mathrm{eV}^{21}$ ). The overall appearance of the spectrum thus suggests a $C_{2 v}$ ground state for $\ln _{2} P$, consistent with the ground states predicted for isovalent $\mathrm{Ga}_{2} \mathrm{As}$ and $\ln _{2} \mathrm{Sb}$ ground states. As mentioned above, this means that $\ln _{2} P^{-}$most likely has a $C_{2 v}$ geometry as well.

We now consider the modes to which the two observed frequencies comespond. The $\omega$, $=204 \mathrm{~cm}^{-1}$ frequency is close to the InP diatomic frequency of $257.9 \mathrm{~cm}^{-1}$ determined by Weltner ${ }^{12}$ by inirared spectroscopy of $\ln P$ isolated in rare-gas matrices, so it is mast likely due to refative In-P motion, namely the symmetric stretch. The $\omega_{2}=47 \mathrm{~cm}^{-1}$ frequency then must correspond to the bend vibration. The low frequency for this mode suggests it is primarily due to relative In-In motion. In ' $x$ ct, this frequency is considerably lower than the calculated $\ln _{2}$ ground state frequency, ${ }^{21}-100 \mathrm{~cm}^{-1}$, suggesting very weak bonding between the two indium atoms in $\ln _{2} P$. Weltner also observed an infrared transiion at $249.3 \mathrm{~cm}^{-1}$ which was assigned to the $v_{3}$ antisymmetric stretch mode of $I n_{2} P$. In a triatomic $C_{2 v}$ molecule with weak binding between the end atoms, the antisymmetric stretch frequency is typically higher than the symmetric stretch, so our assignment of the $v_{1}$ mode and Weltner's assignment of the $v_{3}$ mode appear consistent.

While it is straightforward to assign the main progressions and determine neutral vibrational frequencies for the active modes, it is more difficult to determine the anion freque icies and assign the sequence and hot band progressions. To do so, it is useful to simulate the spectrum for an assumed set of anion and neutral frequencies. This is done within the Franck-Condon approximation, where the intensity of a transition between vibrational levels $v^{\prime \prime}$ and $v^{\prime}$ of the anion and neutral, respectively, is assumed proportional to the Franck-Condon factors:

$$
1\left(v^{\prime} \leftarrow v^{\prime \prime}\right) \propto\left|\left\langle\chi^{\prime}\right| \chi^{\prime \prime}\right) \mid
$$


The vibrational wave functions, $\chi$, are taken to be a product of a Morse oscillaior wave function corresponding to the symmetric stretch, and two harmonic oscillator wave functions corresponding to the symmetric bend and antisymmetric stretch nomal modes. The anion and neutral symmetric mode wave functions are displaced from one another along their corresponding normal coordinates until the observed peak intensities are matched. Our simulations assume that the vibrational modes in the anjon and neutral are separable and parallel. These are certainly reasonable approximations since we are not trying to extract detailed geometric displacements from our analysis.

In the simulations shown as the bottom trace in Figure (9.3), the following frequencies were used: $\omega_{1}^{\prime \prime}=295 \mathrm{~cm}^{-1}, \omega_{1}^{\prime}=207 \mathrm{~cm}^{-1}\left(\omega_{1} \chi_{1}^{\prime}=3 \mathrm{~cm}^{-1}\right)_{1} \mathrm{~T}_{1}=150 \mathrm{~K} ; \omega_{2}^{\prime \prime}=100 \mathrm{~cm}^{-1}, \omega_{2}^{\prime}=$ $47 \mathrm{~cm}^{-1}, \mathrm{~T}_{2}=70 \mathrm{~K} ; \quad \omega_{3}^{\prime \prime}=223 \mathrm{~cm}^{-1}, \omega_{3}^{\prime}=249.3 \mathrm{~cm}^{-1}$ (Weltner's tentative value from matrix work $^{12}$ ) $T_{3}=140 \mathrm{~K}$. The simulation matches the experimental peak spacings quite well (the peak indicated with the arrow has not been fit in the simulation). The peak assignments indicated on the combs in Figure (9.3) were determined by this simulation, where $v$ corresponds to $v_{2}^{\prime}$. Most of the structure observed in the spectrum are attributed to transitions from vibrationally cold anions. The individual peak assignments are listed explicitly in Table (9.1).

Since there were relatively few intense peaks originating from vibrationally excited anion levels, chocsing the anion frequencies for the simulation and assigning the hot band and sequence band structure was not straightforward. The anion symmetric stretch frequency $\left(\omega_{1}{ }^{\prime \prime}=\right.$ $295 \mathrm{~cm}^{-1}$ ) was chosen such that the hot band positions between 519 to $525 \mathrm{~nm}$ could be reasonably matched, treating them as the $2_{0}^{v} 1$, hot band progression. It is not a uniquely determined frequency, as the spectrum could have been fit satisfactorily using 270 or $317 \mathrm{crn}^{-1}$ (i.e., displaced by \pm one half of a bend quantum) for $\omega_{1}^{\prime \prime}$. Overall, however, this is the most reasonable assignment for these transitions. The frequency of $295 \mathrm{~cm}^{-1}$ is 100 high for these transitions to originate from the $v_{2}{ }^{\prime \prime}=1$ anion level, and transitions of the type $2_{0}^{\circ} 3_{1}^{0}$ are not allowed because the $v_{3}$ mode is not totally symmetric. 
We next consider the progression assigned as the $2_{0}^{\nu} 3$, sequence band progression. This progression is displaced $27 \mathrm{~cm}^{-1}$ to the blue of the $2_{0}^{u}$ progression, indicating that the anion frequency in the mode responsible for this structure is $27 \mathrm{~cm}^{-1}$ less than the neutral frequency. so our assignment implies that $\omega_{3}^{\prime \prime}-\omega_{3}^{\prime}=-27 \mathrm{~cm}^{-1}$. Using Weltner's value for $\omega_{3}^{\prime}$ yields $\omega_{3}^{\prime \prime}=$ $223 \mathrm{~cm}^{-1}$. This number is uncertain, however, because the origin of the $2_{0}^{v} 3_{1}^{1}$ progression is difficult to pinpoint. If, for example, the true origin is $20 \mathrm{~cm}^{-1}$ to the red of the $2_{0}^{\nu}$ progression instead of $27 \mathrm{~cm}^{-1}$ to the biue, this would mean that $\omega_{3}{ }^{\prime \prime}=\omega_{3}^{\prime}=20 \mathrm{~cm}^{-1}$ and that $\omega_{3}{ }^{\prime \prime}=269 \mathrm{~cm}^{-1}$. We prefer the lower value but acknowledge it may be incorrect.

The altemate assignment of this progression as the $2{ }_{i}^{v+1}$ hot band progression was rejected for the following reason. We found that the profile of the $2_{0}^{\mathrm{u}}$ progression was best fit assuming a much greater anion $\omega_{2}^{\prime \prime}$ frequency than the $47 \mathrm{~cm}^{-1}$ neutral frequency. When we attempted to fit the spectrum assuming comparable frequencies, the simulated progression was considerably more extended than the observed spectrum. For example, the nomal coordinate displacement could be adjusted until the simulated $2_{0}^{2}$ peak was the most intense of the $2_{0}^{0}$ progression, as in the spectrum, but the simulated $2_{0}^{\prime}$ and $2_{0}^{3}$ transitions were much more intense relative to the $2_{0}^{2}$ transition than what is observed. However, using a much greater anion frequency, i.e. a narrower anion bend potential, the anion wave function is more localized, causing it to have good overlap with fewer neutral wave functions. This yields a narrower progression. The anion frequency in the simulation had to be at least $100 \mathrm{~cm}^{-1}$ in order to reproduce the experimental intensity distribution, implying considerably stronger bonding between the indium atoms.

Given this, it seems surprising that there is no obvious hot band structure that we can attribute to $v_{2}$. However, we found that when the anion symmetric bend frequency was chosen to be a near multiple of the neutral frequency, such as $100 \mathrm{~cm}^{-1}$, the simulation would show no distinct sequence or hot bands in $v_{2}$ (except for possibly the peak indicated with the asterisk, which is fit as a 2, sequence band in the fit presented). The $100 \mathrm{~cm}^{-1}$ anion $v_{2}$ frequency we 
chose for the presented spectrum cannot be considered definitive; the spectral profile could also be fit satisfactorily assuming $\omega_{2}{ }^{\prime \prime}=150 \mathrm{~cm}^{-1}$.

Our overall assignments thus indicate that the symmetric stretch and bend frequencies are higher in the anion than the neutral, with the biggest change occurring in the $v_{2}$ mode. If our assignments are correct, they inıply considerably stronger bonding between the two indium atoms in the anion as compared to the neutral. In the Discussion section it will be shown that this is consistent with the expected anion and neutral molecular orbital occupancies.

\section{2) Approximation of the geometry differences between the anion and neutral}

Since we do not know the exact form of the normal coordinates or the geometries for either the anion or the neutral, we cannot quantitatively determine the geometry differences between them. We can, however, obtain an estimate of the change in In-In bond distance between the anion and neutral, assuming the $v_{2}$ node is primarily an In-In relative motion, which seems likely given the low frequency. The $2_{0}^{\nu}$ progression was fit using an In diatomic, with a neutral trequency of $47 \mathrm{~cm}^{-1}$ and an anion frequency of $100 \mathrm{~cm}^{-1}$, and an absolute bond length difference of approximately $0.2 \mathrm{~A}$ (the In-In distance is presumably smaller in the anion). This is a small change, considering that the calculated In-ln bond distance in $\ln _{2} S b$ is $4.6 \AA$. Assuming an In-P bond distance $0.1 \dot{A}$ greater than the bulk bond distance of $2.54 \dot{A}$ (the In-Sb bond distance in $/ n_{2} S b$ is calculated to be approximately $0.1 \dot{A}$ greater than the bulk bond distance) and a neutral In-In bond distance of $4.6 \hat{A}$, this corresponds to a bond angle change from $112^{\circ}$ in the anion to $120^{\circ}$ in the neutral. This is, however, a crude approximation, and should be treated with appropriate caution.

B. $\ln P_{2}-\pi n P_{2}$

Based on the discussion at the beginning of this section, we expect $\ln \mathrm{P}_{2}{ }^{-}$and $\ln \mathrm{P}_{2}$ to have $C_{2 v}$ symmetry. Therefore, we will only discuss the InP $P_{2}^{-} Z E K E$ spectrum in terms of $C_{2 v} \leftarrow$ 
$\mathrm{C}_{2 v}$ transitions. Both bands in the $\mathrm{InP}_{2}-$ spectrum are much more congested and complicated than band $X$ in the $\ln _{2} P^{-}$spectrum, and spectral simulations of both bands $X$ and $A$ in the $\ln _{2}{ }^{-}$ spectrum have proven unsatisfactory since the intensity profiles of both bands appear anomalous. Nonetheless, the simulations have aided in sorting through the numerous sequence bands and combination bands observed in the spectra, and will now be discussed in some detail.

\section{1) Band $X$ vibrational structure and peak assignments}

As discussed in the results section, one can extract two vibrational frequencies for the $X$ state of neutral $\ln P_{2}$ from the ZEKE spectrum in Figure $(9.5): \quad \omega_{1}^{\prime}=478 \mathrm{~cm}^{-1}$ and $\omega_{2}^{\prime}=190$ $\mathrm{cm}^{-1}$. The frequency for the $v_{2}$ mode is comparable to the In-P symmetric stretch frequency in $\ln \mathrm{n}_{2} \mathrm{P}\left(204 \mathrm{~cm}^{-1}\right)$ and the diatomic $\ln P$ frequency $\left(253 \mathrm{~cm}^{-1}\right)$ and is in good agreement with the calculated $182 \mathrm{~cm}^{-1} \mathrm{v}_{2}$ value for the ${ }^{2} \mathrm{~B}_{2}$ neutral state (Sec. III.B). It is therefore reasonable to assign the $v_{2}$ mode to the $\ln \mathrm{P}_{2}$ symmetric stretch and to expect this mode to consist primarily of In-P relative motion, as is consistent with the calculated normal coordinate for this mode. The $v_{1}$ frequency, higher than but comparable to the calculated $v_{1}$ frequency, is approximately two thirds of the $P_{2}$ triple-bonded ground state $\left({ }^{1} \Sigma_{g}{ }^{+}\right)$frequency, $780 \mathrm{~cm}^{-1}$, suggesting that this mode consists primarily of P-P relative motion between two doubly bonded phosphorous atoms. The frequencies therefore suggest a bonding scheme in which the indium atom is singly bonded to each $P$ atom, and this costs one bond between the two $P$ atoms. This is also consistent with the calculated geometry for the ${ }^{2} \mathrm{~B}_{2}$ ground neutral state, in which the P-P bond distance, 2.16 $A$, is slightly longer than the $P_{2}$ ground state $\left({ }^{1} \Sigma_{g}+\right)$ bond distance, $1.89 A$, but is comparable to the bond distance of the $P_{2}$ first excited state $\left({ }^{1} \Sigma_{y}{ }^{+}\right), 2.12 \dot{A}$.

Band $X$ of the $\operatorname{In} P_{2}$ Z ZEKE spectrum was simulated using Eqn. (9.2) with the above values for $\omega_{1}^{\prime}$ and $\omega_{2}^{\prime} ;$ the remaining anion and neutral frequencies are discussed below. The simulation, shown in the bottom panel of Figure (9.5), appears to reasonably fits the main 
features of band $X$ in the center panel, namely the $2_{0}^{\nu}$ and $1_{0}^{1} 2_{0}^{\nu}$ progressions. The simulation also includes the $1_{0}^{2} 2_{0}^{v}$ progression, not labeled in Figure (9.5), which overlaps the higher energy peaks in the $2_{0}^{v}$ progression. The $v_{2}$ mode clearly shows the most activity, suggesting that the biggest geometry change between the anion and neutral is in the In-P bond length.

The simulations also helped identify two hot band progressions, the $2_{1}^{u+1}$ and $1_{0}^{1} 2_{1}^{u+1}$ progressions, arici the $2_{0}^{\mathrm{V}} 3_{1}{ }_{1}$ sequence band progression. This assignment of the two hot band progressions yields $\omega_{2}=227 \mathrm{~cm}^{-1}$ for the anion, which is in qualitative agreement with the calculated value, $192 \mathrm{~cm}^{-1}$. Based on this, the origin of the 2,1 progression, namely the $2_{1}^{0}$ transition, should appear $227 \mathrm{~cm}^{-1}$ to lower photon energy of the $2_{0}^{\text {u }}$ origin. However, we found that when we scanned to lower photon energy (under the $\mathrm{N}_{2(t)}$-cooled source conditions) the $\mathrm{S} / \mathrm{N}$ in this energy region was such that no additional structure was observed. The $2^{u+1}$ assignment is based on the intensity distribution of the $2_{i}^{v+1}$ progression, which differs from the main $2_{0}^{v}$ progression in that it peaks earlier (at $v=3$ ) and dies off much more rapidly. Such a difference is expected for a hot band because there is a node in the $\mathrm{u}_{2}{ }^{\prime \prime}=1$ wave function where the $v_{2}^{\prime \prime}=0$ wave function is a maximum. Hence the Franck-Condon factors for the two anion levels are quite different. In principle, the intensity distribution in an extended hot band progression should be bimodal. This is seen in the simulated progressions, in which the intensity distribution peaks on both sides of the minimum at $v_{2}^{\prime}=5$. However, the experimental progression does not rise again at higher $U_{2}^{\prime}$; this may be related to the whole progression dying out at lower energy than is observed in the PES [see figure (9.4)], or to the various approximations in our Franck-Condon analysis.

The remaining sequence band progression was assigned to the $2_{0}^{\mathrm{J}} 3{ }_{1}^{\prime}$ progression rather than the $2_{0}^{2} t_{1}^{\prime}$ progression based on the results of the $a b$ initio frequency calculations (Sec. III.B). The calculations predict that the anion $v_{3}$ frequency is on the order of $200 \mathrm{~cm}^{-1}$ lower than the $v_{1}$ frequency. This means that the anion $v_{3}=1$ level should be more populated than the $v_{1}$ $=1$ level, so the sequence band in the $v_{3}$ mode should be more intense. Since the $2_{0}^{u} 3_{1}^{\prime}$ 
progression is displaced $75 \mathrm{~cm}^{-1}$ to the red of the $2_{0}^{*}$ progression, we have $\omega_{3}^{\prime \prime}-c_{3}^{\prime}=75 \mathrm{~cm}^{-1}$, which is in qualitative agreement with the calculated ditference, $126 \mathrm{~cm}^{-1}$.

\section{2) Band $A$ vibrational structure and peak assignments}

By comparing the spectra of band A obtained under different source conditions [top and middle panels of Figure (9.6)], there appears to be only one progression originating from the anion ground state with a peak spacing of $287 \mathrm{~cm}^{-1}$. The neutral vibrational mode associated with this progression cannot be determined based on comparison witt the ab initio frequencies calculated for the ${ }^{2} A_{1}$ excited state, as $287 \mathrm{~cm}^{-1}$ is equally close to both of the calculated symmetric frequencies [see Figure (9.7)]. However, the relative geometries calculated for the anion and the ${ }^{2} A_{4}$ neutrai state suggest that the $v_{2}$ bend, in which the P.P bond elongates while the in-P bond contracts (and vice versa), should be more active than the $v_{1}$ stretch, in which both bonds elongate (or contract). Therefore, the progression has been assigned to $2_{0}^{2}$ transitions, with $\omega_{2}^{\prime}=287 \mathrm{~cm}^{-1}$. It is also interesting po note from Figure (9.7) that in the ground state, $v_{1}$ is primarily a P.P stretch and $v_{2}$ an In-P stretch, while in the ${ }^{2} A_{1}$ state, $v_{1}$ and $v_{2}$ are better described as the symmetric stretch and bend, respectively, and both modes it.volve the relative motion of all three atoms.

The Franck-Condon simulation in Figure (9.6) shows that ond can account for most of the structure in addition to the main progression with two more progressions, the $2_{1}^{\text {b*1 }}$ hot band progression and the $20{ }^{\circ} 3$; sequence band. Explicit peak assignments are listed in Table (9.3). The $2{ }_{1}{ }^{* 1}$ progression was fit assuming $\omega_{2}{ }^{\prime \prime}=227 \mathrm{~cm}^{-1}$ in the anion, the same value found for the $2{ }_{1}^{v+1}$ progression in band $X$. As in band $X$, the intensity distribution peaks earlier for $2_{1}^{\nu+1}$ progression than for the 20 progression. The $2{ }_{0}^{u} 3_{1}^{1}$ sequence bands were so assigned by the same arguments given in the previous section, namely that ab initio calculations predict $\omega_{1}{ }^{\prime \prime}>\omega_{3}$ "for the anion, in which sase $\omega_{3} "-\omega_{3}^{\prime}=-75 \mathrm{~cm}^{-1}$ (the calculated difference is $-99 \mathrm{~cm}^{-1}$ ). Note 
this is opposite in sign to $\omega_{3}^{\prime \prime}-\omega_{3}^{\prime}$ in band $X$. In the simulation, the remaining, lower-intensity peaks were fit by the $2_{0}^{\nu} 3_{2}^{2}, 2_{1}^{v+1} 3_{1}^{1}$ and $2_{2}^{v+2}$ transitions.

The $287 \mathrm{~cm}^{-1} v_{2}$ frequency for this excited state is substantialy higher than the 190 $\mathrm{cm}^{-1}$ frequency observed for the ground state, suggesting that there is more phosphorous relative motion involved in the excited state $v_{2}$ mode. Based on this, we can infer that the P.P bonding is weaker and the In-P bonding is stronger in this first excited state. This is supported by the calculated geometries for the twu neutral states. The $227 \mathrm{~cm}^{-1}$ anion $v_{2}$ frequency fits in nicely as having intermediate bonding properties between the two neutral states, suggestive of the orbital occupancies the anion has in comman with the two neutral states. The molecular orbital configurations for the anion and two neutral states will be discussed in the foliusving section.

A notable feature of band $\mathbf{A}$ is that the peaks in the main progression broaden somewhat for $v_{2}^{\prime} \geq 3$, suggesting there is a perturbation affecting the neutral state that tums on between peaks $2_{2}^{2}$ and $2_{0}^{3}$. This perturbation is clearty different in nature than the intensity falloff compared to the photuelectron spectrum that occurs in band $\mathbf{X}_{1}$ in which the higher-energy peaks mainidined narrow peak widths, and the signal to noise remained quite good. i ine possibility is that the peak broadening is from dissociation to in $+P_{2}$. The dissociation energy for $\operatorname{lnSb}_{2} \rightarrow \ln +\mathrm{Sb}_{2}$ is calculated to be only $1.3 \mathrm{eV} .^{7}$ Since $\mathrm{T}_{\mathrm{e}}$ for the $\operatorname{InP}_{2}$ first excited state is $1.280 \mathrm{eV}$, it is certainly teasible that at least part of band $\mathbf{A}$ lies above the dissociation continuum, thereby causing the broadening of peaks at the higher energy end of the band. The $v_{2}$ normal coordinate would appear to facilitate dissociation; a displacement negative of the coordinate shown in Figure (9.7) gives closer phosphorous atoms combined with a longer In-P distance. Assuming that dissociation is occurring, from the position of peak $\bar{z}_{0}^{3}$, which is sfightly wider than $2_{0}^{2}$, the dissociation energy of $I n P_{2}$ lies between 1.352 and $1.386 \mathrm{eV}$.

Anather explanation for the peak broadening is suggested by Balasubramanian's calculations on $\operatorname{lnSb_{2}}$, which predict that the potential energy curves for the first excited $\left({ }^{2} \mathrm{~A}_{1}\right)$ 
state and ground ${ }^{2} B_{2}$ state cross just above the ${ }^{2} A_{1}$ minimum. These states interact strongly via spin-orbit coupling. If there is a similar curve crossing in $\ln _{2}$, one might expect the energy levels of the excited state that lie above the intersection point to be badly perturbed, possibly leading 10 the broadening seen in band $A$ of our spectrum.

\section{Discussion}

\section{A. $\ln _{2} \mathrm{P}-\mathrm{fn}_{2} \mathrm{P}$ electronic state assignments}

From the vibrational analysis of the $\ln _{2} P^{-}$ZEKE spectum, we concluded that the anion bend frequency is higher than the neutral bend frequency, implying that the in-In bond is stronger in the anion than the neutrej. Moreover, given the low frequency of the bend mode in $1 \mathrm{n}_{2} P\left(\omega_{2}^{\prime}=47 \mathrm{~cm}^{-1}\right)$, the $2_{0}^{\nu}$ progressions in the ZEKE spectrum are quite short, indicating a relatively small displacement along the $v_{2}$ normal coordinate between the anion and neutral. One would like to understand these results in terms of the molecular orbital configurations of $\ln { }_{2} P$ and $\ln _{2} P$ - While no $a b$ initio calculations have been pertormed on either species. Balasubramanian has determined the dominant molecular orbital configurations for the isovalent neutral $\ln _{2} \mathrm{Sb}$ and $\mathrm{Ga}_{2} \mathrm{As},{ }^{7}$ and these serve as a starting point for our discussion.

The ground electronic states of $\mathrm{In}_{2} \mathrm{Sb}$ and $\mathrm{Ga}_{2} A$ s were calculated to have $\mathrm{C}_{2 \mathrm{v}}$ geometries with $a^{2} \mathrm{~B}_{1}$ electronic character from the following valence orbital occupancy:

$$
1 a_{1}^{2} 2 a_{1}^{2} 1 b_{2}^{2} 2 b_{2}^{2} 3 a_{1}^{2}+b_{1}^{1}\left(4 a_{1}^{0}\right)
$$

Qualitative pictures of these orbitals are shown in Figure (9.8); these are adapted from the description by Balasubramanian and the pictures of Mejer et al. ${ }^{9}$ Note that the half-filled $1 b_{1}$ orbital is an out-of-plane orbital with most of its amplitude on the two end atoms. Although 
vibrational frequencies were not deternined for $\mathrm{In}_{2} \mathrm{Sb}$ or $\mathrm{Ga}_{2} \mathrm{As}$, potential energy curves were calculated for the ground and low-lying excited states as a function of bend angle. ${ }^{7}$ The equilibrium bend angles for $\ln _{2} S b$ and $\mathrm{Ga}_{2} A$ s were found to be $104^{\circ}$ and $109.5^{\circ}$, respectively, and both species calculated to have very broad bend potentials, with fairly weak bonding between the two Group III atoms. This is qualitatively consistent with the low bending frequency seen for the $\ln _{2} P$ ground state, and it seems reasonable that the ground state of $\ln _{2} P$ is also a ${ }^{2} \mathrm{~B}_{1}$ state with the valence contiguration of Eqn. (9.3).

The anion electronic orbital occupanty may be determined by adding an electron either to the $1 b_{1}$ orbital, resulting in a ${ }^{1} A_{1}$ state, or possibly the $4 a_{1}$ orital. giving a ${ }^{3} B_{1}$ state. Both of these oritals are bonding between the twi and atoms, so in either case one would expect the anion to be more bent and to have a higher bend frequency than the neutral. The $1 b_{1}$ orbital should be less strongly bonding between the In atoms than the $4 a_{1}$ orbital which, given the relatively small geometry change suggested by the short $2_{0}^{v}$ progression, may tavor the ${ }^{\top} A_{1}$ state for the anion. This is consistent with Balasubramanian's calculations on the excited states of neutral $\ln _{2} S b$ which predict that states formed by promoting an electron from the $3 a_{1}$ or $2 b_{2}$ orbitals to the $1 b_{1}$ orbital are lower in energy than the quartet states formed by promoting the same electrons to the $4 a_{1}$ orbital. However, that the anion $v_{2}$ frequency is substantially greater than the neutral might suggest the ${ }^{3} B_{1}$ state. We hesitate to make a definite anion state assignment lacking more definitive anion frequencies, especially for the $v_{2}$ mode .

Band $\mathbf{A}$ (or lack thereof) Given trat $\ln _{2} \mathrm{P}^{-}$is more bent than the neutral ground state, and that in the $\ln \mathrm{n}_{2} \mathrm{P}^{-}$photoelectron spectrum, the widths of bands $X$ and $A$ are similar, the most likely candidate for the neutral excited state responsible for band $A$ is the ${ }^{2} B_{2}$ state; the ${ }^{2} B_{2}$ state is $\mathrm{ln}_{2} \mathrm{Sb}$ is predicted to have a bend angle of $80^{\circ}$ vs. $104^{\circ}$ tor the ${ }^{2} \mathrm{~B}_{1}$ ground state. ${ }^{7} \mathrm{~A}$ low-lying linear $\left({ }^{2} \Pi_{u}\right)$ state of $\ln _{2} S b$ is also predicted, but the photodetachment transition to this state should be much more extended than band $A$ due to the large change in bend angle between anion and neutral, so it is a less likely candidate for the upper state in band $\mathbf{A}$. 
It still remains unexplained why band $\mathbf{A}$ is not present in the ZEKE spectrum. The selection rules for negative ion threshold photodetachment are more restrictive than for PES, due to the Wigner threshold law. For atomic systems, this dictates that the detachment cross section near threshold goes as:22

$$
\sigma \propto \sigma_{0}\left(E_{h v}-E_{\text {threshold }}\right)^{\ell+1 / 2}
$$

where $\left(E_{h v}-E_{\text {threshodd }}\right)$ is the energy above threshold (i.e., the kinetic energy of the detached electron) and $t$ is the angular momentum of the ejected electron. The result is that only electrons with $t=0$ (s-wave) have a sharply rising cross section near threshold, a necessary condition to observe a ZEKE transition. This has been applied to polyatomic anions by Reed et al., ${ }^{23}$ who point out that only electrons from MO's with the same transformation properties as the $x, y$, or z body-fixed coordinates can undergo s-wave detachment. In a $C_{2 v}$ molecule, all orbitals except those with $a_{2}$ symmetry can detach with $\ell=0$. Hence, from the discussion in the previous section, s-wave detachment is allowed for any one-electron photodetachment transition from a $1_{1}$; anion involving the valence orbitals. This would be true if the anion were a ${ }^{3} B_{1}$ state as well [see Eqn. (9.3)]. In either case one would expect band $A$ in the $\ln _{2} P^{-}$photoelectron spectrum to appear in the ZEKE spectrum as well.

However, as was pointed out by Reed, the selection rule for s-wave detachment is a necessary but not sufficient condition a sharply rising cross section near threshold. Indeed, our work on $\mathrm{Si}_{3}{ }^{-}$showed that several bands in the photoelectron spectrum in which s-wave detachment was allowed did not appear in the ZEKE spectrum. ${ }^{4}$ An important factor here is how much the orbital from which detachment occurs resembies an atomic p-orbital. Of all the orbitals in Figure $(9.8)$, the $1 b_{1}$ appears to have the most atomic p-character, so removal of an electron from this orbital should result in a strong ZEKE signal. In fact, band $\mathrm{X}$ gives the most intense ZEKE signal for any molecular anion we have studied thus far, consistent with its 
assignment as $a^{2} B_{1} \leftarrow{ }^{1} A_{1}$ (neutral $\leftarrow$ anion) transition. However, it is far from clear that the $1 b_{2}$ orbital deviates from an atomic p-obital to a sufficient extent that we could not observe ZEKE signal for the transition to the ${ }^{2} \mathrm{~B}_{2}$ excited state. The determination of conditions sufficient for strong s-wave photodetachment is an interesting and important question for future study as it determines the range of applicability of anion ZEKE spectroscopy.

\section{B. In $P_{2}-\pi n P_{2}$ electronic states}

The experimental anion and neutral vibrational frequencies for $\operatorname{In} P_{2}$ and $I n P_{2}^{-}$are, in general, consistent with the calculated properties of the anion and two neutral states shown in Figure (9.7), supporting our identification of the experimentally observed states with the three calculated states. In this section, we compare the properties of these states to the calculated properties of the analogous ground ${ }^{2} \mathrm{~B}_{2}$ and excited ${ }^{2} \mathrm{~A}_{1}$ states of $\mathrm{GaAs}_{2}$ and $\ln \mathrm{Sb}_{2}{ }^{7,9}$ as well as the ground ${ }^{1} A_{1}$ state of $G_{a A s}-9$ The calculations on these $i_{n} P_{2}$ analogs were considerably more sophisticated and complete than our calculations on $\ln _{2} P$ and $\ln _{2} P^{-}$(although no vibrational frequencies were determined in the earlier studies), so they provide a useful framework for understanding the chemical bonding in $\ln _{2} P$ and $I n_{2} P$ -

The ground ${ }^{2} \mathrm{~B}_{2}$ electronic state orbital occupancy of $\ln \mathrm{P}_{2}, \mathrm{GaAs}_{2}$ and $\ln \mathrm{Sb}_{2}$ is

$$
1 a_{1}^{2} 2 a_{1}^{2} 1 b_{2}^{2} 3 a_{1}^{2} 1 b_{1}^{2} 4 a_{1}^{2} 2 b_{2}^{1}\left(1 a_{2}^{0}\right)
$$

Two close-lying neutral states, the ${ }^{2} \mathrm{~A}_{1}$ and ${ }^{2} \mathrm{~B}_{1}$ states (with calculated $T_{e}$ 's of $0.67 \mathrm{eV}$ and 1.38 $\mathrm{eV}$, respectively, for $\operatorname{lnSb_{2}}$ ) are formed by promoting $\mathrm{a}_{4} \mathrm{a}_{1}$ and $\mathrm{a} 1 \mathrm{~b}_{1}$ electron, respectively, into the $2 b_{2}$ orbital. The ground and first excited electronic orbital occupancies here are those used in the ab initio geometry and frequency calculations described in Sec. III.B for InP 2 [Figure (9.7)]. The anion ${ }^{1} \mathrm{~A}_{1}$ ground state is formed by adding an electron to the $2 \mathrm{~b}_{2}$ orbital of the ${ }^{2} \mathrm{~B}_{2}$ state. 
The ${ }^{3} \mathrm{~B}_{1}$ state with unpaired electrons in both the $2 b_{2}$ and $1 a_{2}$ orbitals was found to lie oniy 0.5 eV higher in energy for $\mathrm{GaAs}_{2}-9$

Figure (9.7) shows that, compared to the $\operatorname{lnP}_{2}{ }^{2} B_{2}$ ground state, the P-P bond distance is longer in the anion and the in-P bond distance is shorter. This reflects the effect the additional electron in the $2 b_{2}$ orbital, which is antibonding between the two phosphorous atoms but bonding between the indium and phosphorous atoms [see Figure (9.8)]. Figure (9.7) shows that the ${ }^{2} A_{1}$ neutral state has a wider bond angle and shorter in-P distance than the neutral ground state; this is consistent with having two electrons in the $2 b_{2}$ orbital but only one in the strongly P-P bonding $4 a_{1}$ orbital.

Our calculated energy separation for the ${ }^{2} B_{2}$ and ${ }^{2} A_{1}$ states of $\ln _{2} P$ is $1.139 \mathrm{eV}$, which is quite close to the observed energy, $1.280 \mathrm{eV}$. This is approximately twice the calculated term energy for the analogous $\mathrm{GaAs}_{2}$ and $\operatorname{lnSb}_{2}{ }^{2} \mathrm{~A}_{1}$ states. ${ }^{7,9}$ The large difference can be understood by considering how the $2 b_{2}$ and $4 a_{1}$ orbitals correlate to the $P_{2}$ diatomic and In atomic orbitals. The $4 a_{4}$ orbital is formed from the $2 \pi_{u}+5 p$ orbitals, while the $2 b_{2}$ orbital correlates to the $2 \pi_{g}+5 p$ orbitals. The energy difference between the $2 \pi_{L}$ and $\pi_{g}$ orbitals in $P_{2}$ is greater than for $\mathrm{As}_{2}$ or $\mathrm{Sb}_{2},{ }^{24}$ causing the $4 \mathrm{a}_{1}-2 \mathrm{~b}_{2}$ splitting to be greater in $\ln \mathrm{P}_{2}$ than for $\mathrm{GaAs}_{2}$ or $\ln \mathrm{Sb}_{2}$.

An interesting issue in these clusters is the degree of covalent vs. ionic bonding in bulk $\ln P$, the bonding is largely ionic, with excess negative charge on the $P$ atoms. However, there are no P.P bonds in crystalline InP, whereas there is strong P.P bonding in $\ln \mathrm{P}_{2}$, suggesting a covalent bonding picture. On the other hand, Balasubramanian calculated the dipole moments associated with the various electronic states and structural isomers of the GaAs and InSb mixed triatomics. ${ }^{7}$ Dipoles of 1.22 $\mathrm{D}$ and 1.64 $\mathrm{D}$ were determined for the ${ }^{2} \mathrm{~B}_{2} \mathrm{C}_{2 v}$ states of $\mathrm{GaAs}_{2}$ and InSb $_{2}$, respectively. As the difference in electronegativity between in and $P$ is greater than for $\mathrm{Ga}$ and $\mathrm{As}$, or In and $\mathrm{Sb}$, it would follow that the dipole of the ${ }^{2} \mathrm{~B}_{2}$ state of $\ln \mathrm{P}_{2}$ is greater than for $\mathrm{GaAs}_{2}$ or $\operatorname{lnSb}_{2}$. This is confirmed by our ab initio calculations, which gave $3.6 \mathrm{D}$ for the ${ }^{2} \mathrm{~B}_{2}$ 
state, and 3.55 for the ${ }^{2} \mathrm{~A}_{1}$ state. Therefore, in spite of the substantial amount of covalent bonding between the two phosphorous atoms in this molecule, the dipole suggests that there is still ionic character along the In-P bond. It is not surprising that the two partially negative phosphorous moieties are so strongly bound in the ground $\ln P_{2}$ state, as $P_{2}^{-}$has been found to be strongly bound $\left(D_{0}=4.88 \mathrm{eV}\right)$ with a bond distance of $1.98 \AA_{1}, 25$ which is only $0.09 \AA$ longer than the neutral bond distance.

\section{Conclusions}

The ZEKE spectra of the two InP triatomics have shown that these very small clusters are remarkably complex. The two triatomics we have investigated are very different from one another. While both having $\mathrm{C}_{2 y}$ geometries, the $\ln _{2} P$ ground state has very weak $\mathrm{In}$-In bonding, an obtuse bond angle, and a very broad potential, in contrast to the $\ln _{2}$ ground state, which has very strong P-P bonding, an acute bond angle, and a fairly steep bend potential. The geometry differences between the anions and neutrals seems to be much more dramatic for In $P_{2}$ than for $\ln _{2} P$.

The $1 n_{2} P-Z E K E$ spectrum shows progressions in both the $47 \mathrm{~cm}^{-1}$ symmetric bend $\left(v_{2}\right)$ and the $204 \mathrm{~cm}^{-1}$ symmetric stretch $\left(v_{1}\right)$ of the $\mathrm{C}_{2 v},{ }^{2} \mathrm{~B}_{1}$ neutral ground state. From the profile of the progressions and the limited sequence and hot band structure, we can infer that all modes of the anion have higher frequencies than the neutral, which is consistent with the anion having the extra electron in the $1 b_{1}$ orbital. The transition to what we believe is the ${ }^{2} \mathrm{~B}_{2}$ state observed in the previously obtained PES is not observed in the ZEKE spectrum.

Based on trends in $v_{2}$ frequencies between the two neutral states and the anion, the ground state of $\ln \mathrm{P}_{2}$ is determined to have ${ }^{2} \mathrm{~B}_{2}$ and a ${ }^{2} \mathrm{~A}_{1}$ first $\ldots$.rited electronic state at 1.280 $\mathrm{eV}$ above the ground state. This is analogous with the calculated ground and first excited states for $\ln \mathrm{Sb}_{2}$ and $\mathrm{GaAs}_{2}$ where the ${ }^{2} \mathrm{~B}_{2}$ ground state and the ${ }^{2} \mathrm{~A}_{1}$ first excited state are separated by 
less than $1 \mathrm{eV}$. The anion ground state has appears to be the ${ }^{1} A_{1}$ state. The ${ }^{2} B_{2}$ ground state of $\mathrm{InP}_{2}$ exhibits much stronger P-P bonding and weaker In-P bonding relative to the first excited ${ }^{2} \mathrm{~A}_{1}$ state, and the anion has bonding properties intermediate of the two neutral states. It is clear from our analysis of the spectra, which was supported by our ab initio calculations, that while the electronic states of this small cluster are fairly close-lying, the vibrational properties are dramatically influenced by simply shifting an electron from the $4 \mathrm{a}_{1}$ orbital to the $2 \mathrm{~b}_{2}$ orbital.

\section{Acknowledgments}

This work was supported under NSF grant no. DMR-9201159. Thanks go to Don Amold for help with calculations and to Mary Mandich for generously supplying us with our first InP rod. 
1J. Faist, F. Capasso. D. L. Sivco, C. Sirtori, A. L. Hutchinson, and A. Y. Cho, Science 264, 553 (1994).

2 E. C. Honea, A. Ogura, C. A. Murray, K. Raghavachari, W. D. Sprenger, M. F. Jarrold, and W. L. Brown, Nature 366, 42 (1993).

${ }^{3}$ T. N. Kitsopoulos, C. J. Chick, A. Weaver, and D. M. Neumark, J. Chem. Phys. 93, 6108 (1990).

4 C. C. Arnold and D. M. Neumark, J. Chem. Phys. 99,3353 (1993).

5 C. C. Amold and D. M. Neumark, J. Chem. Phys. 100, 1797 (1994).

${ }^{6}$ R. O. Janes, Phys. Rev. A 32, 2589 (1985); K. Raghavachari and V. Logovinsky, Phys. Rev. Lett. 55, 26 (1985); G. Pacchioni and J. Koutecky, J. Chem. Phys. 84, 3301 (1986); A. Slanina, Chem. Phys. Lett. 131, 420 (1986); K. Balasubramanian, Chem. Phys. Lett. 135, 283 (1987); C. M. Rohtfing and K. Raghavachari, Chem. Phys. Lett. 167, 559 (1990); R. Foumier, S. B. Sinnott, and A. DePristo, J. Chem. Phys. 97, 4149 (1992).

7 K. Balasubramanian, J. Chem. Phys. 87, 3518 (1987); K. K. Das and K. Balasubramanian, J. Chem. Phys. 94, 6620 (1991).

8 AlP and MgS: M. A. Al-Laham, G. W. Trucks, and K. Faghavachari, J. Chem. Phys. 96, 1137 (1992);GaAs. M. A. Al-Laham and K. Raghavachari, Chem. Phys. Lett. 187, 13 (1991).InP (stoichiometric clusters): K. Raghavachari, personal communication.

9 U. Meier, S. D. Peyerimhoff, and F. Grein, Chem. Phys. 150, 331 (1991).

10 L. Lou, L. Wang, L. P. F. Chibante, R. T. Laaksonen, P. Nordlander, and R. E. Smalley, J. Chem. Phys. 94, 8015 (1991); L. Lou, P. Nordlander, and R. E. Smalley, ibjd. 97, 1858 (1992).

11 R. M. Graves and G. E. Scuseria, J. Chem. Phys. 95, 6602 (1991).

12 S. Li, R. J. Van Zee, and W. Weltner, Jr., J. Phys. Chem. 98, 2275 (1994).

${ }^{13}$ C. Xu, E. deBeer, D. W. Arnold, C. C. Arnold, and D. M. Neumark, to be published. 
14 K. D. Kolenbrander and M. L. Mandich, J. Chem. Phys. 92, 4759 (1990); Phys. Fev. Lett. 65, 2169 (1990); K.-D. Finnen, K. D. Kolenbrander, A. M. DeSantolo, and M. L. Mandich, J. Chem. Phys. 96, 4088 (1992).

${ }^{15}$ T. N. Kitsopoulos, C. J. Chick, Y. Zhao, and D. M. Neumark, J. Chem. Phys. 95,5479 (1991).

${ }^{16}$ C. C. Amold, Y. Zhao, T. N. Kitsopoulos, and D. M. Neumark, J. Chem. Phys. 97,6121 (1992).

17 T. N. Kitsopoulos, I. M. Waller, J. G. Loeser, and D. M. Neumark, Chem. Phys. Lett. 159, 300 (1989); C. C. Amold, Y. Zhao, T. N. Kitsopoulos, and D. M. Neumark, J. Chem. Phys. 97, 6121 (1992).

18 T. G. Dietz, M. A. Duncan, D. E. Powers, and A. E. Smalley, J. Chem. Phys. 74, 6511 (1981).

19 K. Müller-Dethlefs, M. Sander, and E. W. Schlag, Z. Naturforsch 39a, 1089 (1984); Chem. Phys. Lett. 12, 291 (1984); K. Müller-Dethlefs and E. W. Schlag, Annu. Rev. Phys. Chem. 42, 109 (1991).

20 Gaussian 92, Revision C, M.J. Frisch, G. W. Trucks, M. Head-Gordon, P. M. W. Gill, M. W. Wong, J. B. Foresman, B. G. Johnson, H. B. Schlegel, M. A. Robb, E. S. Replogle, R. Gomperts, J. L. Andres, K. Raghavachari, J. S. Binkley, C. Gonzalez, R. L. Martin, D. J. Fox, D. J. Defrees, J. Baker, J. J. P. Stewart, and J. A. Pople, Gaussian, Iric., Pittsburg, PA. 1992.

21 K. Balasubramanian and J. Q. Li, J. Chem. Phys. 88, 4979 (1988).

22 E. P. Wigner, Phys. Rev. 73, 1002 (1948).

23 K. J. Reed, A. H. Zimmerman, H. C. Andersen, and J. I. Brauman, J. Chem. Phys. 64, 1368 (1976).

${ }^{24} \mathrm{G}$. Hertzberg. Molecular Spectra and Molecular Structure, Vol. I. Spectra of Diatomic Molecules. Robert E. Krieger Publishing Company. Malabar, Florida, 1989. p. 561, p. 566 and p. 504 give that the dissociation energies of the $\mathrm{As}_{2}$ and $\mathrm{Sb}_{2}$ ground ${ }^{1} \Sigma_{\mathrm{g}}{ }^{+}$state are lower than that of the $P_{2}{ }^{1} \Sigma_{g}{ }^{+}$state. 
25 J. T. Snodgrass, J. V. Coe, C. B. Freidhoff, K. M. McHugh, and K. H. Bowen, Chem. Phys. Lett. 122, 352 (1985). 
Table (9.1) Peak positions and assignments for the $\ln _{2} P^{*}$ ZEKE spectrum.

\begin{tabular}{|c|c|c|}
\hline $\begin{array}{l}\text { Peak Position } \\
\text { (nm) }\end{array}$ & $\begin{array}{l}\text { Energy from origin } \\
\left(\mathrm{cm}^{-1}\right)\end{array}$ & $\begin{array}{l}\text { Assignment } \\
\left(v_{1}^{\prime} v_{2}^{\prime} v_{3}^{\prime} \leftarrow v_{1}{ }^{\prime \prime} v_{2}^{\prime \prime} v_{3}{ }^{\prime}\right)\end{array}$ \\
\hline 524.45 & -291 & \\
\hline 523.85 & -269 & \\
\hline 523.36 & -251 & \\
\hline 522.63 & -225 & \\
\hline 521.97 & -200 & \\
\hline 521.28 & -175 & \\
\hline 520.77 & -156 & \\
\hline 520.09 & -131 & \\
\hline 519.43 & -107 & \\
\hline 517.76 & .45 & $010 \leftarrow 010$ \\
\hline 516.57 & 0 & Origin; EA $=2.400 \mathrm{eV}$ \\
\hline 515.85 & 27 & $011 \leftarrow 001$ \\
\hline 515.34 & 46 & $010 \leftarrow 000$ \\
\hline 514.15 & 91 & $020 \leftarrow 000$ \\
\hline 513.46 & 118 & $031 \leftarrow 001$ \\
\hline 512.90 & 139 & $030 \leftarrow 000$ \\
\hline 512.15 & 168 & $041 \leftarrow 001$ \\
\hline 511.65 & 187 & $040 \leftarrow 000$ \\
\hline 511.26 & 201 & $100 \leftarrow 000$ \\
\hline 510.39 & 235 & $050 \leftarrow 000$ \\
\hline 510.00 & 250 & $110 \leftarrow 000$ \\
\hline 509.25 & 279 & $060 \leftarrow 000 ; 121 \leftarrow 001$ \\
\hline 508.81 & 296 & $120 \leftarrow 000$ \\
\hline 507.63 & 341 & $130 \leftarrow 000$ \\
\hline 506.50 & 385 & $140 \leftarrow 000$ \\
\hline 506.25 & 395 & \\
\hline 505.03 & 443 & \\
\hline 504.48 & 464 & \\
\hline 503.97 & 485 & $220 \leftarrow 000$ \\
\hline 502.82 & 530 & $230 \leftarrow 000$ \\
\hline 501.73 & 573 & $240 \leftarrow 000$ \\
\hline 500.17 & 635 & $310 \leftarrow 000$ \\
\hline
\end{tabular}


Table (9.2) Peak positions and assignments for band $X$ in the InP $_{2}-$ ZEKE spectrum.

\begin{tabular}{|c|c|c|}
\hline $\begin{array}{l}\text { Peak Position } \\
\text { (n:m) }\end{array}$ & $\begin{array}{l}\text { Relative energy } \\
\left(\mathrm{cm}^{-1}\right)\end{array}$ & $\begin{array}{l}\text { Assignment } \\
\left(v_{q}^{\prime} v_{p}^{\prime} v_{q}^{\prime} \leftarrow v_{q}{ }^{\prime \prime} v_{p}^{\prime \prime} v_{q}{ }^{\prime \prime}\right)\end{array}$ \\
\hline 771.35 & -78 & $020 \leftarrow 020$ \\
\hline 769.25 & -42 & $010 \leftarrow 010$ \\
\hline $\begin{array}{l}766.76 \\
759.98\end{array}$ & $\begin{array}{l}0 \\
116\end{array}$ & $\begin{array}{l}\text { origin; EA = } 1.617 \mathrm{eV} \\
030 \leftarrow 020 ; 011 \leftarrow 001\end{array}$ \\
\hline 757.68 & 156 & $020 \leftarrow 010$ \\
\hline 755.94 & 187 & $010 \leftarrow 000$ \\
\hline 749.41 & 302 & $021 \leftarrow 001 ; 040 \leftarrow 020$ \\
\hline 746.96 & 346 & $030 \leftarrow 010$ \\
\hline 745.23 & 377 & $020 \leftarrow 000$ \\
\hline 741.10 & 451 & $041 \leftarrow 011$ \\
\hline 739.06 & 489 & $031 \leftarrow 001$ \\
\hline 736.59 & 534 & $040 \leftarrow 010$ \\
\hline 734.50 & 573 & $030 \leftarrow 000$ \\
\hline 733.3 & 595 & \\
\hline 729.0 & 675 & $041 \leftarrow 001$ \\
\hline 726.3 & 726 & $050 \leftarrow 010$ \\
\hline 724.4 & 763 & $040 \leftarrow 000$ \\
\hline 721.6 & 816 & $051 \leftarrow 001 ; 130 \leftarrow 010$ \\
\hline 719.2 & 862 & $051 \leftarrow 001 ; 120 \leftarrow 000$ \\
\hline 716.7 & 911 & $060 \leftarrow 010$ \\
\hline 714.6 & 952 & $050 \leftarrow 000$ \\
\hline 711.6 & 1011 & $140 \leftarrow 010$ \\
\hline 709.8 & 1046 & $130 \leftarrow 000$ \\
\hline 707.1 & 1100 & $070 \leftarrow 010$ \\
\hline 705.1 & 1140 & $060 \leftarrow 000$ \\
\hline 702.1 & 1201 & $150 \leftarrow 010$ \\
\hline 700.2 & 1240 & $140 \leftarrow 000$ \\
\hline 695.80 & 1330 & $070 \leftarrow 000$ \\
\hline 690.00 & 1451 & $150 \leftarrow 000$ \\
\hline 686.70 & 1520 & $080 \leftarrow 000$ \\
\hline 682.10 & 1619 & $160 \leftarrow 000$ \\
\hline 678.11 & 1705 & $090 \leftarrow 000 ; 240 \leftarrow 000$ \\
\hline 673.56 & 1804 & $170 \leftarrow 000$ \\
\hline 669.37 & 1897 & $0100 \leftarrow 000 ; 250 \leftarrow 000$ \\
\hline 665.07 & 1994 & $180 \leftarrow 000$ \\
\hline 660.94 & 2088 & $0110 \leftarrow 000$ \\
\hline 656.97 & 2179 & $190 \leftarrow 000$ \\
\hline 652.65 & 2281 & $0120 \leftarrow 000$ \\
\hline 649.0 & 2366 & $1100 \leftarrow 000$ \\
\hline 645.0 & 2462 & $0130 \leftarrow 000$ \\
\hline 641.0 & 2559 & $1110 \leftarrow 000$ \\
\hline 637.0 & 2657 & $0140 \leftarrow 000$ \\
\hline 633.4 & 2746 & $1120 \leftarrow 000$ \\
\hline 629 & 2856 & $0150 \leftarrow 000$ \\
\hline
\end{tabular}


Table (9.3) Peak positions and assignments for band $A$ in the $\operatorname{lnP}_{2}-$ ZEKE spectrum.

\begin{tabular}{|c|c|c|}
\hline $\begin{array}{l}\text { Position } \\
\text { (nm) }\end{array}$ & $\begin{array}{l}\text { Relative } \\
\text { Energy } \\
\left(\mathrm{cm}^{-1}\right)\end{array}$ & $\begin{array}{l}\text { Assignment } \\
\left(v_{1}^{\prime} v_{2}^{\prime} v_{3}^{\prime} \leftarrow v_{1}^{\prime \prime} v_{p}^{\prime \prime} v_{3}^{\prime \prime}\right)\end{array}$ \\
\hline 435.21 & -386 & \\
\hline 432.20 & $-\varepsilon 26$ & $000 \leftarrow 010$ \\
\hline 431.00 & -161 & $0+0 \leftarrow 020$ \\
\hline 429.84 & -99 & $020 \leftarrow 030$ \\
\hline 429.49 & -80 & \\
\hline 428.03 & 0 & origin; $T_{n}=1.280 \mathrm{eV}$ \\
\hline 426.86 & 64 & $010 \leftarrow 010$ \\
\hline 425.86 & 119 & $020 \leftarrow 020$ \\
\hline 425.73 & 126 & \\
\hline 425.49 & 139 & \\
\hline 424.32 & 204 & $030 \leftarrow 030$ \\
\hline 422.79 & 289 & $010 \leftarrow 000$ \\
\hline 421.74 & 348 & $020<-010$ \\
\hline 420.42 & 423 & $030 \leftarrow 020$ \\
\hline 420.14 & 439 & $021 \leftarrow 011$ \\
\hline 419.10 & 498 & $\begin{array}{l}040 \leftarrow 03 n ; 031 \leftarrow 021 \\
022 \leftarrow 011, \text { etc. }\end{array}$ \\
\hline 417.71 & 577 & $020 \leftarrow 000$ \\
\hline 416.72 & 634 & $030 \leftarrow 001$ \\
\hline 416.37 & 654 & $021 \leftarrow 001$ \\
\hline 415.42 & 709 & $\begin{array}{l}040 \leftarrow 020 ; 031 \leftarrow 011 ; 022 \leftarrow \\
002\end{array}$ \\
\hline 414.03 & 790 & $050 \leftarrow 030 ; 041 \leftarrow 021 ;$ etc. \\
\hline 412.80 & 862 & $030 \leftarrow 000$ \\
\hline 411.62 & 931 & $031 \leftarrow 001$ \\
\hline 410.54 & 995 & $050 \leftarrow 020 ; 041 \leftarrow 011$, etc. \\
\hline 408.08 & 1142 & $040 \leftarrow 000$ \\
\hline 403.45 & 1423 & $050 \leftarrow 000$ \\
\hline 399.15 & 1690 & $060 \leftarrow 000$ \\
\hline 394.91 & 1959 & $070 \leftarrow 000$ \\
\hline 390.51 & 2245 & $080 \leftarrow 000$ \\
\hline
\end{tabular}


Figure (9.1) Thresiold photodetachment apparatus. The inset shows a detail of the cluster source in which the InP rod translates and rotates in a slot (1) and is intersected by pulses from the molecular beam (2) and Nd:YAG laser (3) in a Vespel piece (4). The copper clustering channel (5) is cooled by a copper tube carrying $\mathrm{N}_{2(\lambda)}$ (6).

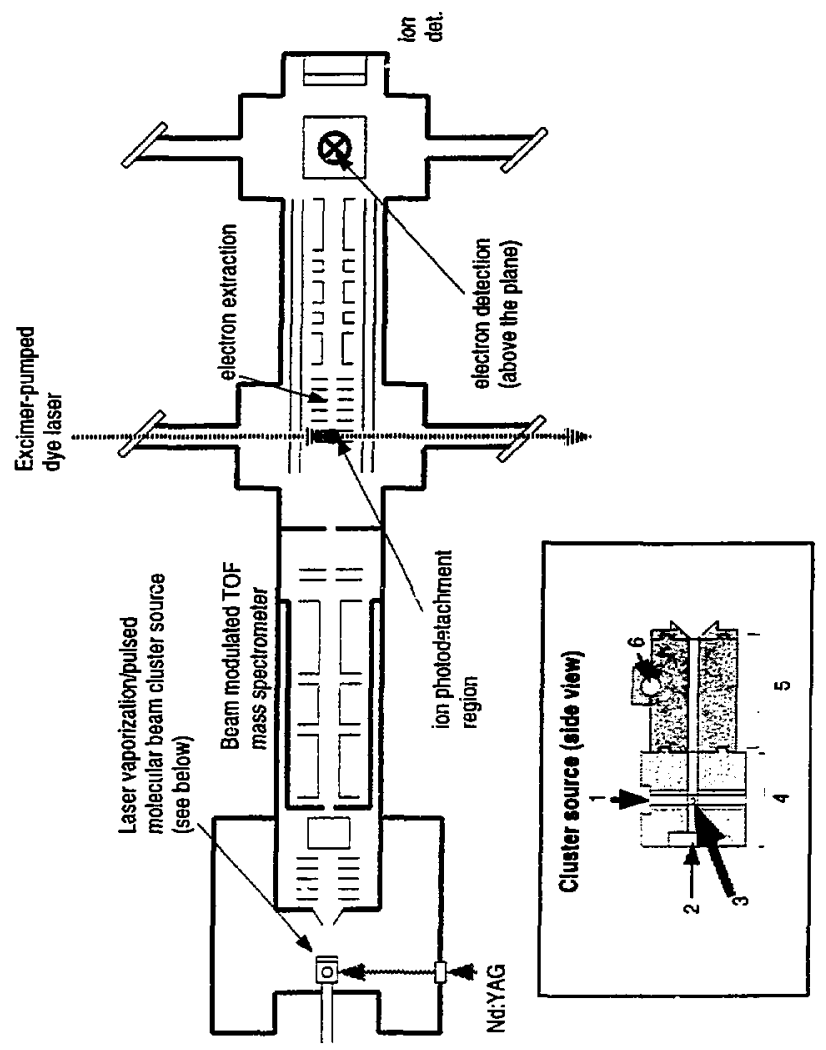


Figure (9.2) ZEKE spectrum (solid line) superimposed onto the $266 \mathrm{~nm}$ (4.66 eV) photoelectron spectrum (dotted line) of $\ln _{2} P^{*}$.

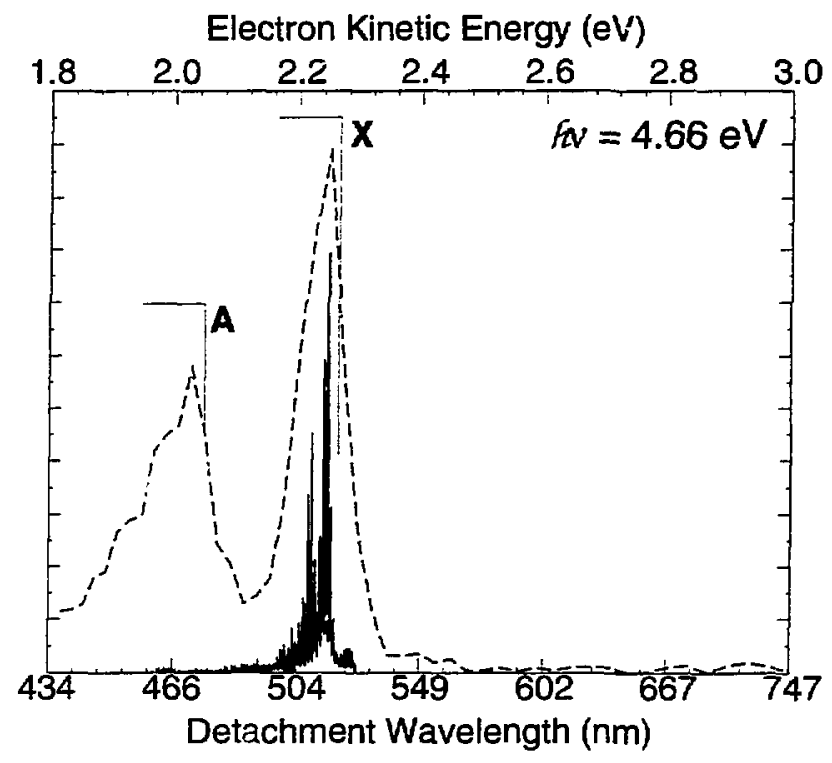


Figure (9.3) ZEKE spectrun, of $\ln _{2} P$ - The upper trace shows data taken using ions generated with the liquid nitrogen-cooled clustering channel, and the center trace with the piezoelectric valve source. The bottom trace is a spectral simulation (see text).

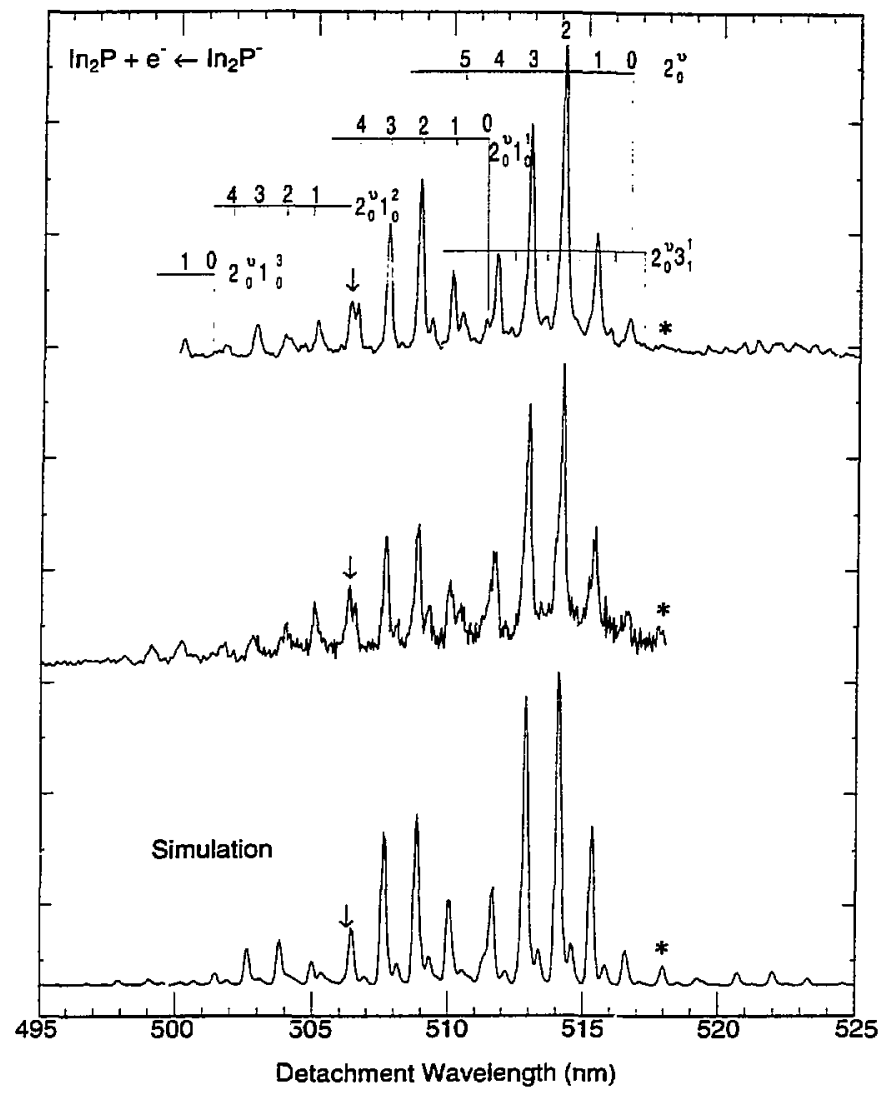


Figure (9.4) Bottom panel: $266 \mathrm{~nm}$ (4.66 eV) photoelectron spectrum of $\ln P_{2}$ :. Upper left:

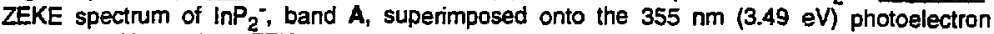
spectrum. Upper right: ZEKE spectrum of $\ln _{2} \mathrm{P}_{2}$, band $X$, superimposed onto the $532 \mathrm{~nm}(2.33$ eV) photoelectron spectrum.
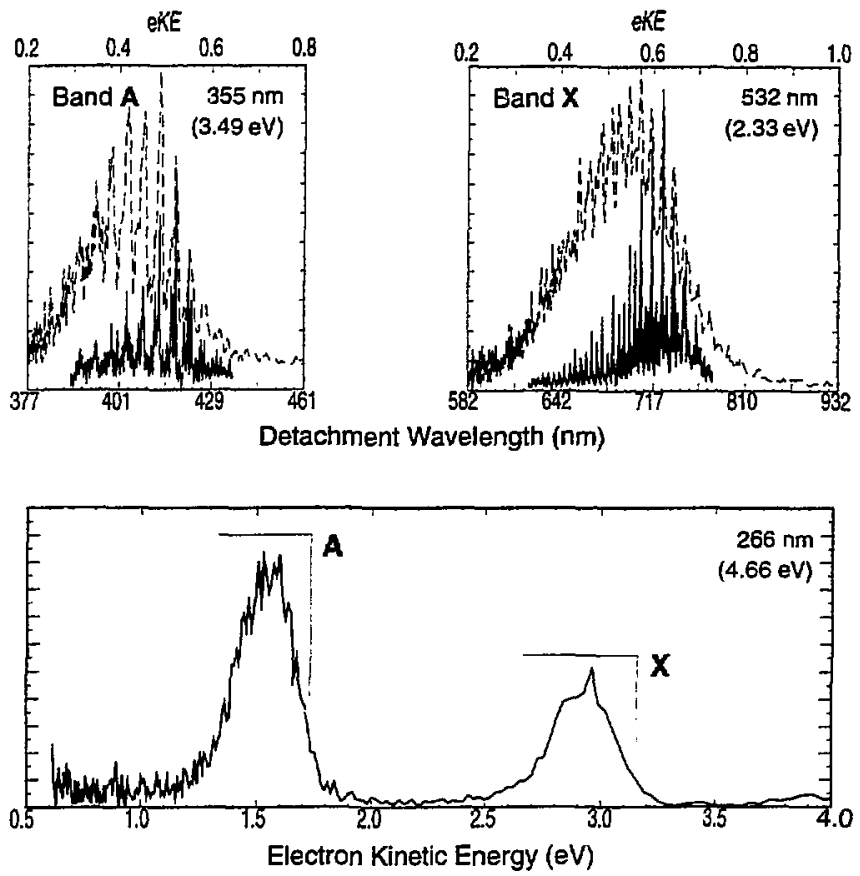
Figure (9.5) ZEKE spectrum of $I n P_{2}^{-}$to the neutral ground state (band $X$ ) obtained under liquid nitrogen-cooled source conditions (upper panel) and ambient source conditions (middle panel). The Franck-Condon simulation is shown in the bottom parlel.

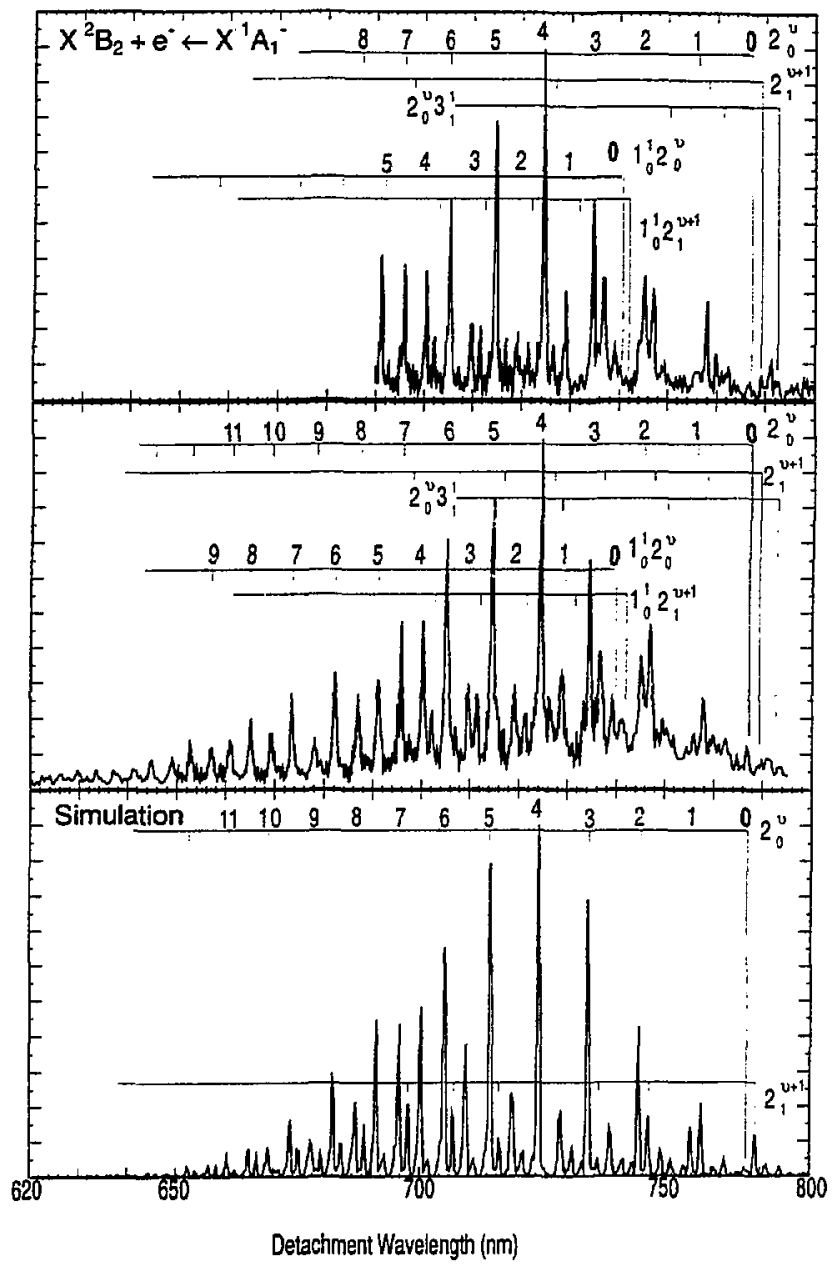


Figure (9.6) ZEKE spectrum of $\operatorname{lnP}_{2}^{-}$to the first excited neutral state (band $A$ ) obtained under liquid nitrogen-cooled source conditions (upper panel) and ambient source conditions (middle panel), and the Franck-Condon simulation (bottom panel).

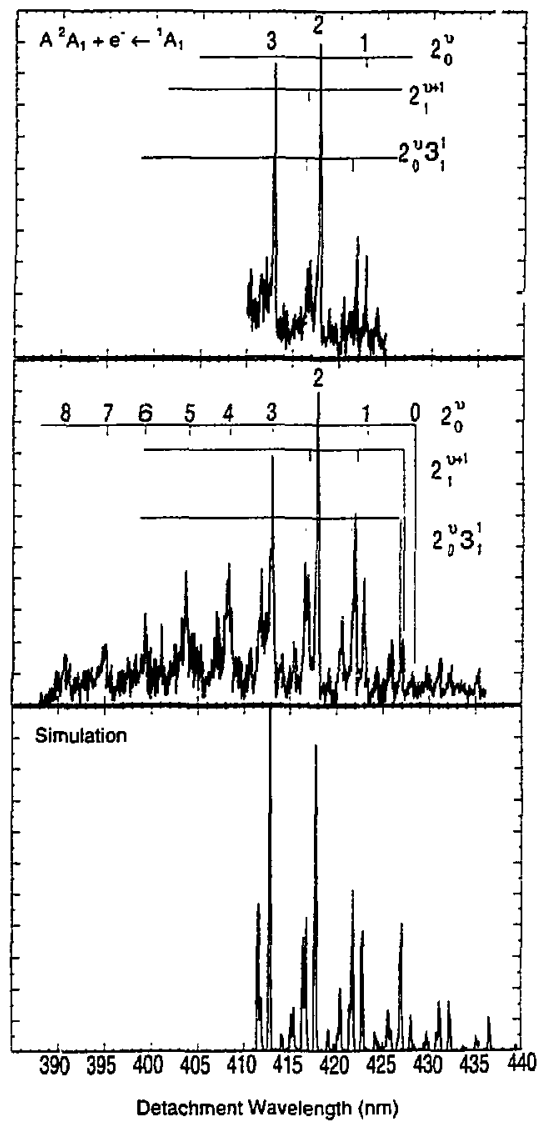


Figure (9.7) Geometries and normal coordinates of $\operatorname{InP_{2}} / \mathrm{InP}_{2}$ - from MP2LANL1DZ calculations.

$\ln \mathrm{P}_{2}{ }_{\mathrm{B}_{2}}$ ground state:
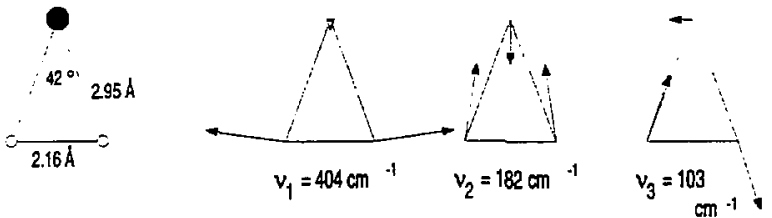

$\ln P_{2}^{2} A_{1}$ first excited state:
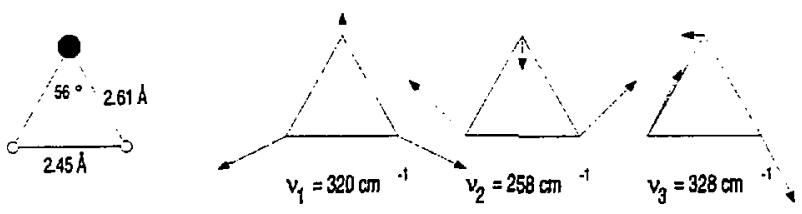

$\ln P_{2}^{-1} A_{1}$, anion ground state:
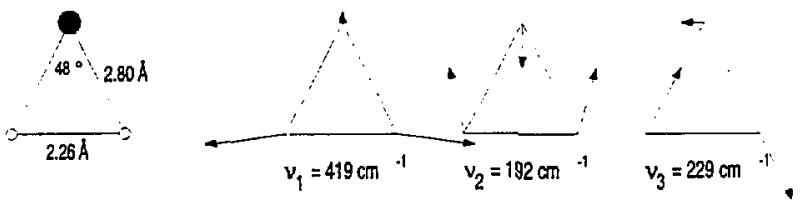
Figure (9.8) Qualitative representations of the high-lying molecular orbitals of $\ln _{2} P$ and $\ln P_{2}$.

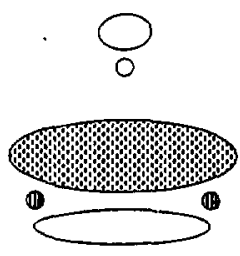

$4 a_{1}$

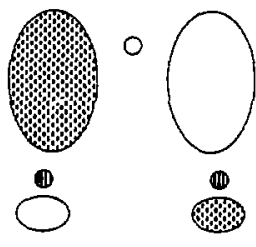

$2 b_{2}$

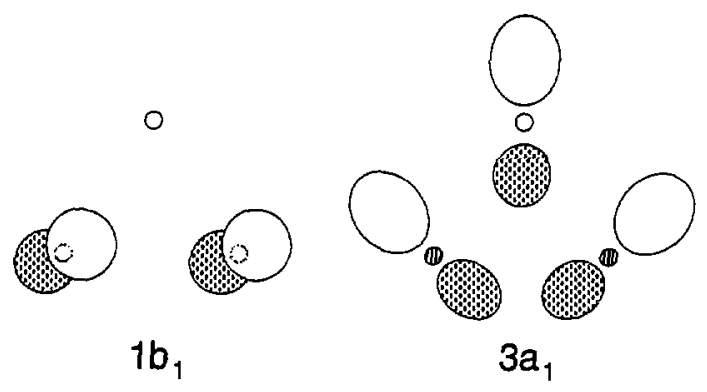




\section{Chapter 10. Study of small gallium arsenide clusters using negative ion photodetachment techniques}

Abstract

The electronically resolved anion photoelectron spectra (PES) of $\mathrm{Ga}_{x} \mathrm{As}_{y}{ }^{-}(x=1,2$; $y=$ $1,2,3)$ and the vibrationally-resolved PES of the GaAs diatomic are presented along with the anion zero electron kinetic energy (ZEKE) spectrum of $\mathrm{Ga}_{2} \mathrm{As} \mathbf{s}^{-}$. The PES of $\mathrm{Ga}_{x} \mathrm{As} \mathrm{s}_{y}{ }^{-}$, which show transitions to the ground and low-lying excited states of the neutral clusters, reveal the

electronic similarity with the analogous $\ln _{x} P_{y}$ clusters. The higher-resolution PES of GaAs* shows transitions originating from both the close-lying ${ }^{2} \Pi$ and $2 \Sigma^{+}$anion states to the triplet and low-lying singlet states of the neutral diatomic. The ZEKE spectrum of $\mathrm{Ga}_{2} \mathrm{As}^{-}$shows two vibrational progressions with characteristic frequencies of $50 \mathrm{~cm}^{-1}$ and $47.0 \mathrm{~cm}^{-1}$ corresponding to the lowest frequency mode $\left(v_{2}\right)$ in two states of the neutral. The two neutral states are separated by approximately $160 \mathrm{~cm}^{-9}$, and appear to be the bent and linear components of a Renner-Teller pair. 


\section{Introduction}

In the ongoing effort to experimentally characterize small, pure group IV semiconductor clusters, relatively little attention has been focused on the mixed group III-V clusters. The impetus is certainiy there; group III-V semiconductors are important to the fast microelectronics industry, with applications in exotic devicas such as If light detectors and semiconductor injection lasers. ${ }^{1}$ The structures and electronic properties of mixed clusters composed of only several atoms present an interesting focus for both experimentalists and theoreticians, as more close-lying structural permutations are available to a binary system than to a pure atomic system. Ab initio studies on mixed systems such as small GaAs, InSb, and AIP clusters have predicted that the various structural permutations for a given cluster can be comparable in energy, and each structure can have multiple close-lying electronic states.2.3,4,5.6 However, cluster experiments that could test calculations are inherently difficult for several reasons, the most forbidding of which is the lack of mass-specificity of most cluster sources. Recause of this, most of the spectroscopic information accrued on small semiconductor clusters has been from mass-selective experiments. $7,8,9,10,11,12$

As an extension of our previous work on small $\ln F$ clusters, ${ }^{8,9}$ this paper presents preliminary results obtained on small GaAs clusters using anion photoelectron spectroscopy (PES) as well as the zero electron kinetic energy (or ZEKE) spectrum of $\mathrm{Ga}_{2} \mathrm{As}^{-}$. Negative ion photodetachment techniques are particularly well-suited to smakl cluster study, as ions are intrinsically mass-selectable, thereby eliminating any ambiguity regarding species identification. Moreover, by detaching an electron from a valence orbital of a cluster anion, both the ground ano Jw-lying excited states of the neutral are probed. Anion PES measures the kinetic energy distribution sf photoelectrons (eKE) in anion detachment of the type

$$
\left.A_{x} B_{y}\left(T_{a}, v_{n}^{\prime}\right)+e-(e K E) \leftarrow h v \text { (fixed) }\right)+A_{x} B_{y}^{-}\left(T_{e}, v_{n}^{\prime \prime}\right), \quad \text { Eqn. (10.1) }
$$


and is generally sensitive to all one-electron transitions between anion and neutral levels having term energies, $T_{\theta}$ and vibrational levels, $v_{n}^{\prime}$, accessible with the photon energy. The resolution afforded by our apparatus is approximately $80 \mathrm{~cm}^{-1}$, which is adequate to resolve electronic (and often vibronic) transitions.

Substantially higher resolution $\left(3 \mathrm{~cm}^{-1}\right)$ can be obtained using negative ion ZEKE spectroscopy, which is sensitive to transitions of the type

$$
A_{x} B_{y}\left(T_{\theta}, v_{n}^{\prime}\right)+e^{-}(e K E=0, \ell=0) \leftarrow h v(\text { tuned })+A_{x} B_{y}^{-}\left(T_{e}, v_{n}^{\nu}\right), \quad \text { Eqn. (10.2) }
$$

where $\ell$ is the angular momentum of the photoelectron, and is related to the molecular orbital from which it is detached. The requirement of $\ell=0$ (s-wave electron) is due to the behavior of the photodetachment cross section near detachment threshold which has been explored by Wigner ${ }^{13}$ for atoms and Reed et al. ${ }^{14}$ for polyatomics. The Wigner threshold law dictates that, near threshold, the cross section goes as

$$
\sigma \propto \sigma_{o}\left(E_{h \downarrow}-E_{\text {throsthold }}\right)^{\ell+1 / 2} .
$$

Therefore, the photodetachment cross section rises shaply at a detachment threshold only for $\ell$ $=0$, so the ZEKE technique is sensitive only to s-wave electrons. Because not all transitions otserved in the lower-resolution PES will be observed in the ZEKE spectrum, the application of both techniques is required for the most complete characterization of these species. We have previously applied the two techniques to the study of small silicon, ${ }^{10,11}$ germanium, ${ }^{12}$ and InP8.9 clusters.

There have been several other experimental studies on small III-V mixed clusters, most of which involve some mass-selectivity. Mandich's photodissociation spectroscopy of $\ln _{x} P_{y},{ }^{15}$ 
showed that clusters containing an even number of atoms exhibited absorption similar to the band gap of bulk InP, while the odd-numbered clusters generally absorbed at lower photon energies. Smailey and coworkers obtained the ultraviolet PES of $\mathrm{Ga}_{x} A s_{y},{ }^{16}$ and the resonant two-photon ionization (R2PI) studies of the GaAs diatomic performed by Morse and coworkers characterized the $X^{3} \Sigma$ and a high-lying $(-3 \mathrm{eV}){ }^{3} \Pi_{r}$ state. ${ }^{17}$ However, even the mass-specific experiments on small GaAs clusters can be confounded because of the proximal masses of $\mathrm{Ga}$ and As atoms. Moreover, the two isotopes of $\mathrm{Ga}$ ( 69 and 71 amu) tend to spread out and congest the mass spectra for those clusters containing more than four atoms. For this reason, the UPES of $\mathrm{Ga}_{\mathrm{x}} \mathrm{As}{ }_{\mathrm{y}}{ }^{-}$by Smalley were mass-resolved only for the total number of atoms in a given cluster, and the electronic structure for any having more than two atoms was nc: uniquely determined in those studies. ${ }^{16}$

In addition to the above experiments are the (not mass-selective) $\varepsilon_{\text {a zctronic and }}$ vibrational absorption work done by Weliner and coworkers on the $\ln (P, A s, S b)^{18}$ and $G a(P, A s$, Sb) ${ }^{19}$ mixed diatomics trapped in rare-gas matrices. The absorption work on GaAs, in particular, determined the vibrational frequency of the ${ }^{3} \Sigma^{\cdot}$ ground state of the GaAs diatomic along with several trequencies assigned to the two mixed tratomics. GaAs is isoelectronic with the more recently experimentally characterized $\mathrm{Ge}_{2}$ and $\mathrm{Si}_{2},{ }^{9,10}$ and has been the subject of several $a b$ initio studies by Raghavachari, ${ }^{2}$ Balasubramanian, ${ }^{20}$ and Bruna and coworkers. ${ }^{21}$ As with the pure Group IV diatomic analogs, a ${ }^{3} \Pi$ excited state is predicted to be very low-lying, within $2000 \mathrm{~cm}^{-1}$ of the ${ }^{3} \mathrm{\Sigma}^{-}$state in the case of GaAs. There are also three singlet states expected to lie approximately $0.5 \mathrm{eV}$ to higher energy having ${ }^{1} \Pi,{ }^{1} \Sigma$, and ${ }^{1} \Delta$ character, in order of increasing energy. The vibrational frequency of the ground 35 state determined by Morse and coworkers $\left(\omega_{e}=215 \mathrm{~cm}^{-1}, \omega_{\mathrm{e}} x_{\mathrm{\theta}}=3 \mathrm{~cm}^{-1}\right)^{17}$ and subsequently observed by weltner in his matrix work ${ }^{19}$ are in reasonable agreement with the calculated irequency. ${ }^{20}$ The several other frequencies observed in Weltner's studies of GaAs trapped in the rare-gas matrices which were 
assigned to the mixed triatomics are consistent with the predicted ground state $\mathrm{C}_{2 \mathrm{v}}$ structures predicted for these species, 3,4 and are further supported by isotopic shift effects.

In this work, we present preliminary electronically-resolved PES of individually massresolved $\mathrm{Ga}_{x} \mathrm{As}_{y}^{-}(x=1,2 ; y=1,2,3)$ clusters. The PES apparatus is presently being modified to dramatically enhance the mass resolution, and PES for these and larger clusters with more indepth analysis of the size-dependent electronic structures of the $\mathrm{Ga}_{x} A s_{y}$ clusters will be presented at a later time. The band structures of the sı:aller species presented here are compared to the previously obtained PES of the analogous $\ln _{x} P_{y}-$ clusters and the $a b$ initio calculations available for these species. The vibrationally-resolved PES of the GaAs diatomic, which is isoelectronic germanium dimer, is also discussed. In addition, the substantially higherresolution anion ZEKE spectrum of $\mathrm{Ga}_{2} \mathrm{As}^{-}$presented below shows extended, low-frequency (- $50 \mathrm{~cm}^{-1}$ ) vibrational progressions in what appears to be two very close-lying states of the neutral.

\section{Experiment}

The operation of the PES and the ZEKE spectrometer are similar in principle. In both experiments, an internally cold beam of cluster anions is generated in a laservaporization/pulsed-molecular beam source based on that developed by Smalley. ${ }^{22}$ The negative ions are then mass-selected and photodetached, but the electron detection schemes are different.

\section{A. Photoelectron spectrometer}

The negative ion PES has been described in detail elsewhere, ${ }^{23}$ but the basic operation is as follows. A beam of coid gallium arsenide anions is generated by vaporizing the surface of 
a translating and rotating GaAs rod with $4.6 \mathrm{~mJ} / \mathrm{pulse}$ output of the second harmonic output of a (pulsed) Nd:YAG operated at $20 \mathrm{~Hz}$ repetition rate. The resulting plasma is entrained in a pulse of $90 \% \mathrm{Ne} / 10 \%$ He carrier gas from a Trickl-type ${ }^{24}$ piezoelectric beam valve, and expanded through a clustering channel into the source vacuum chamber of the apparatus. The cluster anions are extracted into a time-of-flight mass spectrometer where they are accelerated to $1 \mathrm{keV}$ and separate by mass. The ion beam is intersected by a second YAG laser beam, which is timed such that it photodetaches only the cluster ions of interest. A small $f$ dction (approximately $0.4 \%$ ) of the resulting photoelectrons is detected with a $70 \mathrm{~mm}$ diameter microchannel plate detector at the end of a $1 \mathrm{~m}$ field-free drift tube that intersects the mass spectrometer drift tube at the interaction region. The photoelectron kinetic energies (eKE) are determined from the electron flight time. The instrumeital resolution is eKE-dependent, starting from B-10 meV for photoelectrons with 0.65 eKE, and degraúing as (eKE) $)^{3 / 2}$.

\section{B. ZEKE spectrometer}

A detailed description of the anion ZEKE spectrometer can bE found elsewhere, ${ }^{25}$ but briefly, the anions are generated in a similar manner to that described above, except that the clustering channel is cooled using liquid nitrogen, and a General Valve solenoid-iype pulsed molecular beam valve is used in lieu of the piezoelectric beam valve. Use of the $\mathrm{N}_{2(\ell)}$-cooled clustering channel was found to enhance the vibrational cooling in both the InP mixed triatomic clusters and $\mathrm{Si}_{4} \cdot 9$ After the expansion through the clustering channel, those anions passing through a $2 \mathrm{~mm}$ skimmer are colinearly accelerated to $1 \mathrm{keV}$ and separate out by mass in a $1 \mathrm{~m}$ time-of-flight tube.

The mass separated ions then enter a detector region where they are selectively detached using a (pulsed) excimer-pumped tunable dye laser in a field-free region. The dye laser is scanned through the detachment continuum of the cluster anion of interest, 
photoelectrons detached with excess eKE scatter away from the ion bunch, while those with nearly zero kinetic energy (threshold electrons) stay proximal to the remaining ions and neutrals. After severa! hundred nanoseconds, a weak (typicaily 2 V/cm) extraction field is applied, and those energetic electrons scattered of the ion beam axis are blocked by a series of apertures between the detachment region and the electron detector. The on-axis energetic electrons and the threshoid electrons have different arrival times ai the electron detector due to their spatial separation in the extraction field, and the threshoid electre's are selectively collected using a gated integration scheme. This selective detection of threshold electrons, based on techniques developed by Müller-Dethlefs for the photoion „zation of neutrals, yields an energy resolution of as good as $3 \mathrm{~cm}^{-1}$.

The electrun signal is normalized to the ion current and detachment laser power, and averaged over 1500 laser shots per point. The dyes used to obtain the ZEKE spectrum of $\mathrm{Ga}_{2} \mathrm{As}^{-}$were Coumarin 450, Coumarin 480 , and Coumarin 501 .

\section{Results}

\section{A. PES of $\mathrm{Ga}_{\mathbf{x}} \mathrm{As}_{\mathbf{y}}=$}

Figure (10.1) shows six PES of $\mathrm{Ga}_{x} \mathrm{As}_{y} \cdot(x=1,2 ; y=1,2,3)$ obtained using a photon energy of 4.'j57 eV. The eKE is given by

$$
\text { eKE }=h v-E A-E^{(0)}-E^{(-)} \text {, }
$$

where $h$ is the photon energy, EA is the electron of the neutral cluster, and $E^{(0)}$ and $E^{(-)}$are the internal (electronic plus vibrational) energies of the neutral and anion states, respectively. Therefore, the features in the spectra at highest eKE correspond to trarritions to the lowest 
energy leveis of the neutral. Electron affinities for the neutral species were take! to be the onset of the high eKE edge of the highest EKE spectral feature (i.e., low neutral internal energy) which. per Eqn. (10.4), reflects the energy between the ground anion level and the ground neutral ievel. These values are summarized in Table (10.1). Each of the spectra also show several resolved or partially-resolved peaks, each of which represents an electronic transition from the anion (presumably) ground state to different electronic states of the neutral. Approximate term energies $\left(T_{e}\right)$ for these various states are also included in Table (10.1). None of the spectra show any vibrational structure at this photon energy.

It should be noted that on the $\mathrm{Ga}_{2} \mathrm{As}^{-}$and $\mathrm{Ga}_{2} \mathrm{As}_{3}^{-}$spectra, there are low-intensity peaks at approximately $2.8 \mathrm{eV}$ and $3.0 \mathrm{eV}$ eKE. These peaks have opposite polarization dependencies, and the peak at $2.8 \mathrm{eV}$ happens to be enhanced in the magic angle spectra shown. These features are due to two-photon processes in which fragmentation is followed by detachment of the daughter ion, and are therefore unrelated to the $\mathrm{Ga}_{2} \mathrm{As}$ and $\mathrm{Ga}_{2} \mathrm{As}_{3}$ electron affinities. Very similar features were observed in some of the $\ln _{x} P_{y}-$ spectra, ${ }^{8}$ and were tentatively assigned to $\mathrm{In}_{2}$ - detachment, which at first suggests that structure seen here may be due to $\mathrm{Ga}_{2}$ - detachment. There has been no experimental determination of the electron affinity of $\mathrm{Ga}_{2}$, but it has been calculated to be $1.04 \mathrm{eV}_{1}^{26}$ although this value increases to $1.25 \mathrm{eV}$ when more electrons are included in the calculation. 20 This value is low compared to the $1.6 \mathrm{eV}$ detachment energy suggested by the two-photon peak, either implying that the previous assignment is wrong, or that the calculated value is low. The splitting between the two peaks, $0.2 \mathrm{eV}$, is somewhat higher than the $0.05 \mathrm{eV}$ calculated splitting between the $X^{3} \Pi_{u}$ and ${ }^{3} \Sigma_{g}-10 w-$ lying states in $\mathrm{Ga}_{2}$ neutral (As with GaAs, the singlet states are predicted to lie $0.5 \mathrm{eV}$ higher than the triplet states, but the predicted anion ground state, ${ }^{4} \Sigma_{g}{ }^{-}$, cannot access the singlet states). However, the EA's of atomic $\mathrm{Ga}(0.30 \pm 0.15 \mathrm{eV})$ and $\mathrm{As}(0.81 \pm 0.03 \mathrm{eV})^{27}$ are even lower than the predicted EA of $\mathrm{Ga}_{2}$, and there is no other obvious explanation for the structure. 
While the limited range of cluster sizes for we which were able to obtain PES (due to the limitations of the mass spectrometer) precludes our asserting any definite size-dependent trends in the observed electronic band structures, several spectral features are worth being noted. For instance, in the $\mathrm{Ga}_{2} \mathrm{As}_{y} \cdot$ series, $\mathrm{Ga}_{2} \mathrm{As}_{2}$ has a much lower electron affinity than both $\mathrm{Ga}_{2} \mathrm{As}$ and $\mathrm{Ga}_{2} \mathrm{As}_{3}$ [see Table (10.1)]. The even-numbered cluster also has a higher $\mathrm{T}_{\mathrm{e}}$ than the two oddnumbered clusters. More definitive size-dependent and even-odd trends and how they compare with previously obtained $\ln _{x} P_{y}$ - PES spectra will be born out in the larger cluster spectra, and will be the subject of a subsequent anticle.

\section{B. Higher rosolut:an PES of GaAs}

The PES of GaAs obtained using a $2.98 \mathrm{eV}$ photon energy $(416 \mathrm{~nm})$ and three different laser polarizations is shown in Figure (10.2). Electrons ejected from different orbitals generally have different angular distributions relative to the electric field vector of the detachment laser following 28

$$
\frac{d \sigma}{d \Omega}=\frac{\sigma_{\text {total }}}{4 \pi}\left[1+\beta(E)\left(\frac{3}{2} \cos ^{2} \theta-\frac{1}{2}\right)\right] .
$$

Therefore, by obtaining spectra at different laser polarizations, overlapping electronic transitions can more easily be discerned.

The spectra appear to be composed of two vibrationally-resolved bands, both of which are composed of multiple electronic transitions. This spectral profile is familiar; the PES of $\mathrm{Si}_{2},{ }^{10} \mathrm{Ge}_{2},{ }^{12}$ and InP-(8) showed similar band structure due to transitions to the triplet states (lower-lying band) and the singlet states (higher-lying band) from the two close-lying doublet anion states. In all cases, the spacing between the triplet and singlet bands is approximately 0.5 eV. The predicted electronic structure particular to GaAs will be addressed in the discussion 
section. The peak positions (eKE, eV) and relative energies $\left(\mathrm{cm}^{-1}\right)$ are summarized in Table (10.2). Also in Table (10.2), those peaks enhanced in the $\theta=0^{\circ}$ are indicated with a dagger (1) and those enhenced in the $\theta=90^{\circ}$ are indicated with an asterisk $\left({ }^{\circ}\right)$.

The triplet band is particularly congested, and it is clear that multiple electronic transitions are overlapping. However, by considering the difference between the $\theta=0^{\circ}$ and $\theta=$ $90^{\circ}$ spectra, there appear to be at least two and possibly three electronic transitions with different polarization dependencies contributing to the observed structure. The first and most obvinus band is dominated by peak 1 in the $\theta=0^{\circ}$ spectr $\cdot \pi$; it distinctly stands out as a single intense peak with $\theta=0^{\circ}$, while in the $\theta=90^{\circ}$ spectrum, it is buried under peak 2 . The second band is broader and lies to lower eKE, and is more enhanced in the $\theta=90^{\circ}$ spectrum. Most of the intensity of peaks 6 through 15 have appear to be in this second transition; the peaks are not evenly spaced, rather they appear to consist of three transitions separated by 0.06 and $0.07 \mathrm{eV}$, each having a $240 \pm 40 \mathrm{~cm}^{-1}$ vibrational progression. For instance, in the $\theta=0^{\circ}$ spectrum, peaks 11,8 and 5 appear to be the maxima of three $240 \mathrm{~cm}^{-1}$ vibrational progressions, with the spacing between 5 and 8 being $-0.06 \mathrm{eV}$ and the spacing between 8 and 11 being $-0.07 \mathrm{eV}$. This might be what one would expect in a transition to a ${ }^{3} \Pi$ state. A possible third band may contribute to the observed structure and intensity at the higher eKE of the triplet band; at first glance of the $\theta=90^{\circ}$ spectrum, there appears to be only one electronic band, but when the $\theta=$ $90^{\circ}$ spectrum is subtracted from the $\theta=0^{\circ}$ spectrum, there appear to be two bands with slightly different polarization dependencies.

The singlet band is much simpler, and the resolution in eKE $=0.2$ to $0.5 \mathrm{eV}$ region is better than for the higher eKE region. It consists of at least four resolved electronic bands, several of which exhibit vibrational progressions: The first is composed of peaks 19 (probable origin), 21 , and 23 , has a frequency of $285 \pm 20 \mathrm{~cm}^{-1}$, and is enhanced in the $\theta=0^{\circ}$ spectrum. Peak 17 appears to be a hot band associated with this progression. The second is composed of peaks 20 (origin), 22, 24 and 25 , also has a frequency of $285 \pm 20 \mathrm{~cm}^{-1}$, and is enhanced in the 
$\theta=90^{\circ}$ spectrum. The peaks in these two progressions are approximately $60 \mathrm{~cm}^{-1}$ vide at half the maximum (FWHM). The other two bands are at lower eKE, in a range where peak intensities tend to be less reliable. However, in the PES obtained usirıg $3.49 \mathrm{eV}$ detachment energy (not shown), peaks 28 and 29 emerge definitively as the origins of two electronic transitions having opposite angular dependencies, with peak 28 being enhanced in the $\theta=0^{\circ}$ spectrum. Peaks 28 and 29 are separated by $280 \mathrm{~cm}^{-1}$, which is comparable to a GaAs vibration. Therefore, part of the intensity of peak 29 may be due to a vibrational progression originating from peak 28 . There are several less-intense peaks in the $3.49 \mathrm{eV}$ PES that appear to form a $300 \mathrm{~cm}^{-1}$ progression from peak 29. Peaks 27 through 29 are wider $\left(80\right.$ to $100 \mathrm{~cm}^{-1}$, FWHM) than the peaks at higher eKE in the singlet band, which could suggest that there are other transitions overlapping.

\section{ZEKE spectrum of $\mathrm{Ga}_{2} \mathrm{As}$ -}

The ZEKE spectrum of $\mathrm{Ga}_{2} \mathrm{As}^{*}$ is shown as the solid trace in the upper half of Figure (10.3) with the previously discussed PES superimposed (dashed line) on the electron binding energy scale. For future reference, the lower hall of Figure (10.3) shows the PES (dotted line) and ZEKE spectrum (solid line) of isoelectronic $\ln _{2} P \cdot$. Note that only the lowest-lying electronic band in the PES is observed in the ZEKE spectrum, which is the same situation as in the ZEKE spectrum of $\ln _{2} \mathrm{P}$. The ground electronic state of $\mathrm{Ga}_{2} \mathrm{As}$ is predicted to have $\mathrm{C}_{3}$. geometry, and since symmetric modes tend to be the most active in electronic transitions, the $a_{1}$ bend $\left(v_{2}\right)$ and the higher-frequency $a_{1}$ symmetric stretch $\left(v_{1}\right)$ should account for most of the structure observed in the spectrum and described below.

The ZEKE spectrum is shown on an expanded scale in the upper panel of Figure (10.4) as the solid line. It appears to consist of two electronic bands, $X$ and $A$, having different vibrational contours; band $X$ (the lower energy band) has a narrower profile with a $50 \pm 2 \mathrm{~cm}^{-1}$ 
progression, and band A (the higher-lying band) has d broader profile with a $47.0 \pm 0.2 \mathrm{~cm}^{-1}$ progression. These are presumably neutral bend frequencies. Tentative origins of the two bands are indicated with combs labeled $X_{2}$ and $A_{2}$, where the "2" denotes the mode active in the progression, the $v_{2}\left(a_{1}\right)$ symmetric bend, that the comb is indicating. However, these origins, particularly that of band A, cannot be determined definitivaly. Nonetheless, an approximate electron affinity can be detemined from the position of the most intense peak in band $x$, which yields a $2.452 \mathrm{eV}$ electron binding energy. The peaks in the ZEKE spectrum will be referred to as members of progressions indicated by the combs. For example, peak $X_{2}(0)$ is the first peak, or origin of the $X_{2}$ progression, and peak $A_{12}(3)$ is the fourth peak, or third excited level in the $A_{12}$ progression.

Band $X$, in addition to the $50 \pm 2 \mathrm{~cm}^{-1}$ progression, has a hot band, $x_{2}^{\prime}$ found at 259 $\mathrm{cm}^{-1}$ to lower detachment energy of peak $X_{2}(0)$, which also exhibits a $50 \mathrm{~cm}^{-1}$ progression with lower signal to noise. To higher detachment energy, band $X$ overlaps with band $A$, and it is not immediately obvious which peaks belong to which band.

In addition to the most intense $47.0 \pm 0.2 \mathrm{~cm}^{-1}$ progression $\left(A_{2}\right)$ in band $A$ is a lowerintensity $47 \mathrm{~cm}^{-1}$ progression displaced to higher detachment energy. Based on the spacing between the most intense members of each progression, $A_{2}(2)$ and $A_{12}(2)$, the displacement is $171 \mathrm{~cm}^{-1}$, which is likely the vibrational frequency for the symmetric stretch.

\section{Analysis and Discussion}

The approach taken in this section is to first address the $\mathrm{Ga}_{x} \mathrm{As}_{\mathrm{y}}{ }^{-P E S}$ in terms of how they compare to the analogous $\ln _{x} P_{y}$ PES. This comparison will be brief, deferring $m r$ detailed analysis to the more complete set of $\mathrm{Ga}_{x} A s_{y}-$ PES that is fortheoming. The highe, resolution GaAs PES will be discussed in terms of possible transitions between the predicted 
anion and neutral states. Finally, the $\mathrm{Ga}_{2} \mathrm{As}^{-}$ZEKE spectrum will be analyzed and compared to the previously obtained $\ln _{2} P$ - ZEKE spectrum. ${ }^{12}$

\section{A. Electronic structure of $\mathrm{Ga}_{\mathbf{x}} \mathrm{As}_{\mathbf{y}}$}

There is a remarkable similarity between the electronic structures observed in several of the PES of $\mathrm{Ga}_{x} \mathrm{As} \mathrm{s}_{y}$ and their analogous $\ln _{x} \mathrm{P}_{y}{ }^{-}$clusters. ${ }^{8}$ This is perhar 3 a little surprising considering of the difference in polarity between In-P and Ga-As bonds aiong with the obvious atomic size differences: $\mathrm{Ga}$ and $\mathrm{As}$ are comparable in size while In has 1.5 the atomic radius of P. There are also several interesting differences, which along with the similarities, will now be briefly discussed (the GaAs- and $\mathrm{Ga}_{2} \mathrm{As}^{-}$spectra will be addressed in sections $\mathrm{B}$ and $\mathrm{C}$, respectively).

The PES of $\mathrm{GaAs}_{2}$ shows transitions to three electronic states of the neutral. The PES of $\mathrm{InP}_{2}$ showed only two electronic transitions in the energy regies accessed by the same photon energy, in spite of its having a lower EA ( $1.61 \mathrm{eV}$ vs. the $1.83 \mathrm{eV}$ EA of $\mathrm{GaAs}_{2}$ ). ${ }^{8} \mathrm{As}$ was determined from the ZEKE spectrum of $\operatorname{InP} P_{2}$, the ground ${ }^{2} B_{2}\left(\ldots 1 b_{1}{ }^{2} 4 a_{1}{ }^{2} 2 b_{2}\right)$ state of this molecule resembles a doubly bound $P_{2}$ diatomic with an In atom loosely attached in $C_{2 v}$ geometry. The $1.280 \mathrm{eV}$ exsitation to the first excited ${ }^{2} \mathrm{~A}_{1}$ state was effectively moving an electron from a P.F bonding orbital to a P.P antibonding orbital. ${ }^{8}$ Since the bonding between atoms is stronger for lighter atoms than their heavier counterparts, it would then follow that the $\mathrm{T}_{\mathrm{e}}$ of the first excited state in $\mathrm{GaAs}_{2}$ would be smaller than for $\ln \mathrm{P}_{2}$. This was predicted by Meier et al.. ${ }^{4}$ who calculated the ${ }^{2} \mathrm{~B}_{2}-{ }^{2} \mathrm{~A}_{1}$ splitting in $\mathrm{GaAs}_{2}$ to be $0.65 \mathrm{eV}$, which is in reasonable agreement with the observed splitting (the widths of bands $X$ and $A$ in the PES indicates a considerable geometric rearrangement between the anion and both neutral states, so getting a good adiabatic $T_{e}$ from the PES is not straightfonward). Band $B$ is presumably a 
transition to the ${ }^{2} \mathrm{~B}_{1}$ state, but no calculations on the excitation energy for this state are presently available for comparison.

The $\mathrm{GaAs}_{3}-$ PES spectrum is considerably more congested than the $\operatorname{InP}_{3}{ }^{-}$spectrum, ${ }^{8}$ presumably for similar reasons. It seems reasonable that in As-rich GaAs clusters, As-As bonding would dominate (as is the case in $\mathrm{GaAs}_{2}$ ), simply because As atoms have more valence electrons. Again, since electronic stales resulting from As-As bonding schemes should be closer lying than for the states resulting from P-P bonding in P-rich InP clusters, it makes sense that there should be a higher density of states observed in the $\mathrm{GaAs}_{3}{ }^{-}$spectrum than in the $\ln P_{3} \cdot$ spectrum.

The profiles of the $\mathrm{Ga}_{2} \mathrm{As}_{2}{ }^{-}$and $\operatorname{In}_{2} \mathrm{P}_{2}$ - PES are very similar. ${ }^{-}$The differences between the two spectra are subtle; the electronic bands in the $\mathrm{Ga}_{2} \mathrm{As}_{2}^{-}$spectrum are broader, suggesting a greater geometry change between the anion and neutral states than for $\ln _{2} \mathrm{P}_{2}$, the electron aftinity of $\mathrm{Ga}_{2} \mathrm{As}_{2}$ is 0.1 to $0.2 \mathrm{eV}$ higher than the $E A$ of $\ln _{2} \mathrm{P}_{2}$, and the electronic transitions are slightly closer-lying in the $\mathrm{Ga}_{2} \mathrm{As}_{2}{ }^{-}$spectrum. The ground neutral state is predicted to be hombic, with the As atoms on the shorter axis, and having ${ }^{1} \mathrm{~A}_{\mathrm{g}}$ electronic character. This is analogous to the $\mathrm{Si}_{4}$ neutral ground state. ${ }^{11,29}$ The anion is predicted to have ${ }^{2} \mathrm{~B}_{2 g}$ character, which also is analogous to the ground state of $\mathrm{Si}_{4}{ }^{-}$. Therefore, excited states accessed in the PES are likely similar to the excited states of $\mathrm{Si}_{4}$, with band $\mathrm{A}$ being the ${ }^{3} \mathrm{~B}_{3 \mathrm{u}} \leftarrow$ ${ }^{2} \mathrm{~B}_{2 g}$ transition. The $\mathrm{T}_{e}$ 's for the ${ }^{3} \mathrm{~B}_{3 \mathrm{u}}$ state of $\mathrm{Ga}_{2} \mathrm{As}_{2}$ and $\mathrm{Si}_{4}$ are comparable, $0.7 \mathrm{eV}$ for $\mathrm{Ga}_{2} \mathrm{As}$ and $0.82 \mathrm{eV}$ for $\mathrm{Si}_{4}$. This is not so surprising, however, in light of the similarities between the band structure of GaAs and $\mathrm{Si}_{2}$, which is discussed below.

The PES of $\mathrm{Ga}_{2} \mathrm{As}_{3}{ }^{-}$and $\ln _{2} \mathrm{P}_{3}{ }^{-}$again are quite similar. There are presently no calculations available on these larger non-stoichiometric clusters for comparison, but the spectra exemplify how the open-shelled electronic structure of the odd neutral clusters result in higher electron affinities and lower-lying excited electronic states. 


\section{B. Electronic structure of GaAs}

The GaAs diatomic has been the subject of several non-relativistic ab initio studies, all of which conclude that the ground and first excited electronic states are the ${ }^{3} \Sigma^{-}\left(2 \sigma^{2} 1 \pi^{2}\right)$ and ${ }^{3} \Pi$ $\left(2 \sigma^{3} 1 \pi\right)$ states, respectively. ${ }^{2,20,21}$ The ground state was confirmed to be the ${ }^{3} \Sigma^{*}$ state by Morse and coworkers in their R2PI studies on the diatomic. ${ }^{17}$ A vibrational frequency of $215 \mathrm{~cm}^{-1}\left(\omega_{e} x_{e}\right.$ $=3 \mathrm{~cm}^{-1}$ ) was determined for this state as well. No transitions to the $3 \Pi$ state, to our knowledge, have yet been observed. The corresponding singlet states are predicted to lie approximately 0.7 to $2 \mathrm{eV}$ higher in energy. The ' $\Pi\left(2 \sigma^{3} 1 \pi\right)$ state is the lowest-lying of these with calculated $\mathrm{T}_{e}$ 's ranging from $0.74 \mathrm{eV}$ to $0.8 \mathrm{eV}$. The $(1)^{1} \Sigma^{+}$(primarily $\left.1 \pi^{4}\right)$ and ${ }^{1} \Delta\left(2 \sigma^{2} 1 \pi^{2}\right)$ states are predicted to be nearly degenerate at $T_{\theta}=0.9$ to 1.0 , with the $(1)^{1} \Sigma^{+}$state slightly lower in energy. Finally,

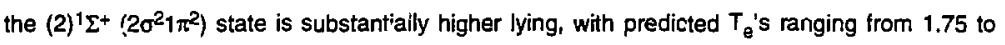
$2.0 \mathrm{eV}$. All of these states are analogous to the lowest-lying triplet and singlet states in isoelectronic $\mathrm{Si}_{2}$ and $\mathrm{Ge}_{2}$ (except for the ordering of several of the lower singlet states) and likely have similar relativistic effects as those observed in the $\mathrm{Ge}_{2}$ studies. 12

The anion has been studied by Balasubramanian, ${ }^{21}$ and, not surprisingly, has similar electronic structure to the anions of silicon and germanium dimers. The ground state is predicted to be the ${ }^{2} \Sigma^{+}\left(2 \sigma 1 \pi^{4}\right)$ state, with the ${ }^{2} \Pi\left(2 \sigma^{2} 1 \pi^{3}\right)$ state lying approximately $0.1 \mathrm{eV}$ higher in energy (the splitting between the two $\Omega$ - components of the ${ }^{2} \Pi$ state is not taken into account in this calculation). All of the previously mentioned neutral states are accessible by one or both of these anion states via a one-electron transition, and a schematic of these transitions is shown in Figure (10.5). Since the spin-orbit coupling constant, $A$, in GaAs' should be roughly $1000 \mathrm{~cm}^{-1}$, the ${ }^{2} \Pi(3 / 2)$ level is probably very close to, if not lower than, the ${ }^{2} \Sigma^{+}$level. We may therefore expect both levels to be populated in the molecular beam.

The transitic to the $(2)^{1} \Sigma^{+}$state is not shown in Figure (10.5). The (2) ${ }^{1} \Sigma^{+}$state lies up to $1 \mathrm{eV}$ higher than the other singlet states, and it will not be observed in the higher-resolution 
PES of GaAs" shown in Figure (10.2) as the $2.98 \mathrm{eV}$ photon energy is not sufficient to access it. However, the 4.66 eV PES shown in Figure (10.1) shows some signal at eKE $=1.0 \mathrm{eV}$ which may be from the $(2)^{1} \Sigma^{+} \leftarrow{ }^{2} \Pi$ transition. $A T_{e}$ of roughly 1.6 to $1.7 \mathrm{eV}$ for this state can be determined from the splitting between this signal and the high eKE edge of the triplet band.

Since the triplet band of the GaAs- in the PES shown in Figure (10.2) is so congested, we can endeavor to analyze the singlet band first, and then make some qualitative comment on the structure in the triplet band. Attempts to obtain the ZEKE spectrum of GaAs- are presently underway, and the results of that experiment should give more definitive assignments of the structure in the triplet band.

The lowest lying singlet state predicted for GaAs is the ${ }^{1} \Pi$ state, which can be accessed from both of the doublet anion states. It therefore seerns logical that the two $295 \pm 20 \mathrm{~cm}^{-1}$ progressions (peaks 19-21-23 and peaks 20-22-24) found at the higher eKE end of the singlet band are from the transitions from the two anion states to the ' $\Pi$ state. The opposite angular dependencies of these two progressions is suggestive; in the similarly obtained PES of $\mathrm{Ge}_{2}{ }^{*}$ and $\mathrm{Si}_{2}$, transitions involving the ejaction of an electron from a $\pi$-type orbital le.g., the ${ }^{1} \Pi \leftarrow{ }^{2} \Sigma^{+}$ transition) were generally enhanced in the $\theta=90^{\circ}$ spectrum while ejection of a $\sigma$-type orbital (e.g., the ${ }^{1} \Pi \leftarrow{ }^{2} \Pi$ transition) were more enhanced in the $\theta=0^{\circ}$ spectrum. We therefore assign peaks $19 . .$. to the ${ }^{1} \Pi \leftarrow^{2} \Pi(3 / 2)$ transition, and peaks 20 ... to the ${ }^{1} \Pi \leftarrow^{2} \Sigma^{+}$transition. Peak 17 , as mentioned previously, appears to be a hot band transition associated with the 19-21-23 progression. The spacing between peak 17 and 19 gives a vibrational frequency of $250 \mathrm{~cm}^{-1}$ for the ${ }^{2} \Pi(3 / 2)$ anion state. The relative frequencies of the ${ }^{2} \Pi(3 / 2)$ anion and ${ }^{1} \Pi$ neutral states are in goid agreement with the predicted values $\left[242 \mathrm{~cm}^{-1}\right.$ for the anion, $280 \mathrm{~cm}^{-1}$ for the neutral from FOCl calculations, Fef. (21)]. The frequency of the $2 \Sigma^{+}$anion state cannot be determined definitively from the spectrum, but it is predicted to be $291 \mathrm{~cm}^{-1}, 21$

The energy splitting between the origins of these two transiticns should give the energy splitting between the ${ }^{2} \Sigma^{+}$and ${ }^{2} \Pi(3 / 2)$ anion states. If peak 19 is in fact the origin of the ${ }^{1} \Pi \leftarrow$ 
${ }^{2} \Pi$ transition and peak 20 the origin of the ${ }^{1} \Pi \leftarrow{ }^{2} \Sigma^{+}$transition, the ${ }^{2} \Pi(3 / 2)$ anion level lies 10 $\mathrm{meV}\left(80 \mathrm{~cm}^{-1}\right)$ above the ${ }^{2} \Sigma^{+}$state. For comparison, in $\mathrm{Ge}_{2}$, the ${ }^{2} \Pi_{\mathrm{u}}(3 / 2)$ was found to lie 274 $\mathrm{cm}^{-1}$ below the ${ }^{2} \Sigma_{\mathrm{g}}{ }^{+}$anion level, but as the spin-abit splitting should be on the order of 700 $1000 \mathrm{~cm}^{-1}$, the ${ }^{2} \Pi_{u}(1 / 2)$ anion level should be several hundred wave numbers above the ${ }^{2} \Sigma_{g}{ }^{+}$ level, and the center of the $\Omega=1 / 2$ and $3 / 2$ levels (the "deperturbed" level) should also lie above the ${ }^{2} \Sigma_{g}+$ state. ${ }^{12}$ The splitting between these two triplet neutral levels for GaAs is predicted to be larger than for $\mathrm{Ge}_{2}$ neutral, ${ }^{20}$ so it would follow that the splitting in the anion levels is larger as well, which is consistent with the observed ordering of the anion levels in GaAs:

The other two electronic transitions, peaks 28 and 29 , could be due to the ${ }^{1} \Sigma^{+} \leftarrow{ }^{2} \Sigma^{+}$ transition and the ${ }^{1} \Delta \leftarrow{ }^{2} \Pi(3 / 2)$ transition. Unless the ${ }^{1} \Delta$ state lies within $80 \mathrm{~cm}^{-1}$ of the ${ }^{1} \Sigma^{+}$ state, the former transitions s'lould be lower-lying. The vibrational frequency calculated for ${ }^{1} \Sigma^{+}$ state is $302 \mathrm{~cm}^{-1}$, while for the ${ }^{1} \Delta$ state it is $220 \mathrm{~cm}^{-1} .20$ Since there are no peaks observed under $280 \mathrm{~cm}^{-1}$ to lower eKE of peak 28 , we assign peak 28 to the origin of the ${ }^{1} \Sigma^{+} \leftarrow{ }^{2} \Sigma^{+}$ transition. This gives that the ${ }^{1} \Sigma^{+}$state is $0.15 \mathrm{eV}\left(1200 \mathrm{~cm}^{-1}\right)$ higher-lying than the ${ }^{1} \Pi$ state. The remaining transition, peak 2.9 , which is found $280 \mathrm{~cm}^{-1}$ to lower eKE of peak 28 , could be the ${ }^{1} \Delta \leftarrow{ }^{2} \Pi(3 / 2)$ transition. However, this assignment is very suspicious since there appears to be no lower-frequency progression extending from peak 29 which would be characteristic of the transition to the ${ }^{1} \Delta$ state. Upon obtaining the ZEKE spectrum of GaAs, the peak assignments will be made more definitively.

Even with the preceding analysis of the singlet band, the assignment of the structure in the triplet band is far from straightforward. What is expected in the triplet band are two s-wave electronic bands [the ${ }^{3} \Sigma^{*} \leftarrow{ }^{2} \Pi(3 / 2)$ transition and the ${ }^{3} \Pi\left(2,1,0^{+}\right) \leftarrow{ }^{2} \Sigma^{+}$transition] and one $p$ wave barid [the ${ }^{3}\left[\left(2,1,0^{ \pm}\right) \leftarrow{ }^{2} \Pi(3 / 2)\right.$ transition]. Only peak 1 in the PES stands out distinctly as a p-wave transition, based on its polarization dependence. Very prelimınary work on the ZEKE spectrum indicates that peak 2 is the origin of an extended $260 \mathrm{~cm}^{-1}$ vibrational progression, while no ZEKE signal is observed under peak 1 . Because $260 \mathrm{~cm}^{-1}$ is substantially 
higher than the known frequency of the ${ }^{3} \Sigma^{-}$state, peak 2 must be the origin of the ${ }^{3} \Pi(2) \leftarrow{ }^{2} \Sigma^{*}$ transition. Peak 1 is roughly $0.02 \mathrm{eV}$ from peak 2 (although this splitting has an uncertainty of $100 \mathrm{~cm}^{-1}$ given the congestion of the spectum) which is on the order of the $2 \Sigma^{+} \cdot 2 \Pi(3 / 2)$ splitting in the anion. It therefore seems reasonable to assign peak 1 to the ${ }^{3} \Pi(2) \leftarrow 2 \Pi(3 / 2)$ electronic hot band transition.

This preliminary assessment leaves several questions unanswered, however. First, it is not clear from the spectrum where the ${ }^{3} \Pi\left(0^{ \pm}\right.$and 1$) \leftarrow{ }^{2} \Pi(3 / 2)$ transitions lie, and why they are not as intense as peak 1 . In the $\mathrm{Ge}_{2}{ }^{-}$spectrum, the ${ }^{3} \Pi\left(0^{+}\right) \leftarrow 2 \Pi(3 / 2)$ transitions had less than half the intensity of the ${ }^{3} \Pi(2) \leftarrow{ }^{2} \Pi(3 / 2)$ transition, but the ${ }^{3} \Pi(1) \leftarrow{ }^{2} \Pi(3 / 2)$ transition had comparable intensity. Second, the ${ }^{3} \Sigma^{-} \leftarrow{ }^{2} \Pi(3 / 2)$ transition is not distinctly observed in the PES. From the work of Morse and Weltner, the vibrational frequency of the ${ }^{3} \Sigma$ - state is approximately $210 \mathrm{~cm}^{-1}$. However, there is $n 0210 \mathrm{~cm}^{-1}$ progression observed in the spectrum; most of the spacings are on the order of $240 \mathrm{~cm}^{-1}$ or more. Geometry calculations on the ${ }^{3} \Sigma$ and $2 \Pi$ states predict that the difference in bond length between these states is approximately $0.2 \dot{A}$. A geometry change of this magnitude should excite a fairly extended progression with the third or fourth member being the most intense. Moreover, this progression should lie to lower detachment energy (higher eKE) than the transitions to the ${ }^{3} \Pi$ state, and very little structure is observed to higher eKE in the PES. However, these issues should be resulved upon obtaining the complete ZEKE spectrum.

\section{Analysis of the ZEKE spectrum of $\mathrm{Ga}_{2} \mathrm{As}$ -}

The similarity of the PES of $\mathrm{Ga}_{2} \mathrm{As}^{\prime}$ and $\mathrm{In}_{2} \mathrm{P}^{-}$[dashed lines, Figure (10.3)] suggests that electronically analogous anion and neutral states are responsible for the two electronic bands in 
both spectra. It is therefore surprising that the ZEKE spectra of $\mathrm{Ga}_{2} \mathrm{As}^{-}$and $\mathrm{In}_{2} \mathrm{P}^{-}$exhibit such distinct differences. The most obvious difference is that there are two very close-lying electronic bands in the ZEKE spectrum of $\mathrm{Ga}_{2}$ As' where only one distinct electronic band is observed in the $\ln _{2} P^{-}$spectrum. The first issue to be addressed is, therefore, whether the two bands reflect transitions from one anion state to two close-lying states in the neutral, or transitions from two close-lying states in the anion to one or two neutral states. Either seems feasible considering the calculated electronic structure of the neutral state.

$A b$ initio calculations on neutral $\mathrm{Ga}_{2} \mathrm{As}$ predict a ${ }^{2} \mathrm{~B}_{1} \mathrm{C}_{2 v}$ ground state with the electronic orbital configuration $\left(1 a_{1}\right)^{2}\left(2 a_{1}\right)^{2}\left(1 b_{2}\right)^{2}\left(2 b_{2}\right)^{2}\left(3 a_{1}\right)^{2}\left(1 b_{1}\right)^{1}\left(4 a_{1}\right)^{0}$ and a Ga-As-Ga bond angle of $111^{\circ} .3$ This configuration represents the bent electronic component of a Renner-Teller pair, for which the linear component, in $C_{2 v}$ terms, has the ...(3a $(3)^{1}\left(1 b_{1}\right)^{2}$ configuration, forming a ${ }^{2} A_{1}$ state, or, the ${ }^{2} \Pi_{y}$ linear state in the $D_{\text {ach }}$ point group. The $3 a_{1}$ and $1 b_{1}$ oritals correlate to the degenerate components of the $\pi_{u}$ orbital in the linear molecule, which has the p-orbitals on all three atoms in phase, and in the bent geometry, the $1 b_{1}$ orbital has a nodal plane in the plane of the molecule. The bent and linear components are predicted to be very close-lying, split by only 0.1 to $0.2 \mathrm{eV}\left(800\right.$ to $1300 \mathrm{~cm}^{-1}$ ), with both having very broad and shallow bend potentials. No calculations or, the anion state of $\mathrm{Ga}_{2} \mathrm{As}$ are yet available, but a possible anion state can be inferred by adding an electron to the distorted, partially-filled $1 b_{1}$ orbital, which results in a ${ }^{1} A_{1}$ state that is isoelectronic with the (predicted) ground state of neutral $\mathrm{Ge}_{3}$. The bond angle of the ${ }^{1} A_{1}$ siate of $\mathrm{Ge}_{3}$ is calculated to be approximately $150^{\circ}$, and if the analogous $\mathrm{Ga}_{2} \mathrm{As}^{-}$state is similarly bent, it could conceivably overlap with the bent and linear components of the neutral Penner-Teller pair, provided all three bend potentials are sufficiently broad.

The analogy between the $\mathrm{Ga}_{2} \mathrm{As}^{-}$anion and neutral $\mathrm{Ge}_{3}$ also gives rise to another possible explanation for the two bands in the ZEKE spectrum. $\mathrm{Ge}_{3}$ is predicted to have a lowlying linear state, having ${ }^{1} \Sigma_{g}{ }^{+}$character (which correlates with the ${ }^{1} A_{1}$ electronic configuration) If the same held tor $\mathrm{Ga}_{2} \mathrm{As}^{\prime}$, and the ${ }^{1} \Sigma_{g}{ }^{+}$state were only several hundred wave numbers higher 
in energy than the ${ }^{1} A_{1}$ state, band $X$ could be an electronic hot band originating from this state, with band $A$ being the ${ }^{2} B_{1} \leftarrow{ }^{1} A_{1}$ transition. This scenario is unattractive for several reasorts. rirst, the excited linear anion state would only overlap with the linear component of the RennerTeiler pair, and transitions to only even quanta in the bend would be allowed. Moreover, very little excitation in the bend would be expected. However, it is possible that the frequency of the linear component of the neutral is $25 \mathrm{~cm}^{-1}$, and that band $X$ is the ${ }^{2} \Pi_{u} \leftarrow{ }^{1} \Sigma_{g}+$ electronic hot band transition. In this case, the splitting between band $X$ and band $A$ would reflect both the splitting between the two anion states and the two neutral states. The other low-lying state predicted for $\mathrm{Ge}_{3}$ is the $\mathrm{D}_{3 n}{ }^{3} \mathrm{~A}_{2}$ state, which correlates to a ${ }^{3} \mathrm{~B}_{2}$ in $\mathrm{C}_{2 \mathrm{v}}$ geometry. This state cannot impact the spectrum, however, since the electronic configurations of the ground and lowlying noi itral states of $\mathrm{Ga}_{2} \mathrm{As}$ can be accessed from a $\mathrm{Ga}_{2} \mathrm{As}^{-}$anion with the ${ }^{3} \mathrm{~B}_{2}$ configuration.

While these arguments based on $a b$ initio geometnies of analogous species are fairly compelling, further convincing is desired before making definitive spectral assignments. We can therefore attempt to simulate the spectrum assuming both scenarios. The spectral simulations are performed within the Frank-Condon approximation, in which we assume that the intensities of individual vibronic transitions are proportional to the Fianck-Condon factors between the anion and neutral vibrational wave functions. The $\mathrm{Ga}_{2} \mathrm{As}$ anion and neutral wave functions are taken to be the product of two harmonic oscillator wave functions corresponding to the $v_{1}$ symmetric stretch $\left(a_{1}\right)$, and the $v_{2}$ symmetric bend $\left(a_{1}\right)$. The $v_{3}$ antisymmetric stretch $\left(b_{2}\right)$ was neglected for simplicity, although it is likely contributing to sequence bands in the observed spectrum.

We periorm simulations in two ways. First, we assume two sets of neutral parameters and one set of anion parameters, in order to simulate the first scenario in which the ground anion state is overlapping with both the bent and linear components of the neutral. Second, we assume one set of neutral frequencies and two sets of anion frequencies, in order to simulate 
the second scenario in which two bent states of the anion are overlapping with only the bent component of the neutral ground state.

The first simulation attempt with the two sets of neutral parameters is shown as the dashed line in the upper panel of Figure (10.4). The two electronic components comprising the simulation are shown as dashed and solid lines in the lower panel of Figure (10.4). For band $X$, the following neutral frequencies were used: $\omega_{1}^{\prime}=160 \mathrm{~cm}^{-1}, \omega_{2}^{\prime}=51.2 \mathrm{~cm}^{-1}$. For band $A, \omega_{1}^{\prime}=$ $171 \mathrm{~cm}^{-1}$ and $\omega_{2}^{\prime}=47 \mathrm{~cm}^{-1}$. For the anion, $\omega_{1}^{\prime \prime}=264 \mathrm{~cm}^{-1}$ and $\omega_{2}^{\prime \prime}=48 \mathrm{~cm}^{-1}$. Of all these frequencies, the $\omega_{2}^{\prime}$ (band $X$ ), $\omega_{2}^{\prime}$ (band $A$ ), $\omega_{1}^{\prime}$ (band $A$ ), and $\omega_{1}^{\prime \prime}$ are obvious from the spectrum. The remaining frequencies are chosen to fit other more subtle feacures in the spectrum. For instance, the $\omega_{y}^{\prime}$ (band $X$ ) was chosen to give appropriate width to the peak labeled as the origin of $X_{12}$, which overlaps with the $A_{2}$ origin.

The $\omega_{2}^{\prime \prime}$ (anion) frequency was chosen for consistency with two features in the spectrum. First is the broadness of the $47 \mathrm{~cm}^{-1}$ progression in band A. The profile of an extended progression is fairly sensitive to the spatial extent of the wave function of the lower state. Since the $47 \mathrm{~cm}^{-1}$ progression in band $A$ is so extended, with five peaks being comparably intense near the maximum intensity of the progression, the anion wave function for this mode must be very broad. In this case, $\omega_{2}^{\prime \prime}=48 \mathrm{~cm}^{-1}$ adequately fit the observed profile. This is in shim contrast with the progression observed in the $\ln _{2} P \cdot Z$ ZKE spectrum [Figure (10.3)], which has a much narrower profile in spite of its extension. The second feature matched by the anion $\omega_{2}^{\prime \prime}=48 \mathrm{~cm}^{-1}$ is the hot band found just to lower detachment energy $\left(19729 \mathrm{~cm}^{-1}\right)$ of the $X_{2}$ origin. Granted, it is not obvious whether the origin of band $X$ is as it is shown in Figure (10.4) or if it is the peak to $50 \mathrm{~cm}^{-1}$ lower detachment energy that has been assigned to a hot band in the preceding simulation. However, the profile of band $X$ could not be matched assuming the latter given $\omega_{2}^{\prime \prime}=48 \mathrm{~cm}^{-1}$. If the anion frequency were higher, the profile of band $X$ could be fit given the lower energy origin, but the more distinct profile of band $A$ would then not be matched. 
While the frequencies chosen for the two neutral states in the preceding simulation are not identical, they are very proximal, which leaves the feasibility that one neutral state is being accessed by two close-lying anion states possible. For the simulation assuming two anion states and one neulral state, the sole neutral frequencies for $v_{1}$ and $v_{2}$ were taken to be 171 $\mathrm{cm}^{-1}$ and $47 \mathrm{~cm}^{-1}$, as they are most definitively determined from the less congested progressions in band A. However, no matter how the anion frequencies were chosen, the confinement to one set of neutral parameters would not yield a good fit. First, the band $X$ progression, while exhibiting similar spacing to the band A progression, is not well matched with a $47 \mathrm{~cm}^{-1}$ frequency. Second, with $\omega_{1}^{\prime}=171 \mathrm{~cm}^{-1}$, the $X_{12}$ origin falls exactly on the $A_{2}$ origin, and no other transitions fill in the several broad peaks where the two bands overlap.

Based on the simulations and the arguments presented at the beginning of this section, we feel confident that the two bands in the ZEKE spectrum are from transitions from ane anion atate to the two components of the Renner-Teller pair of the neutral. Band $X$ is assigned to the ${ }^{2} B_{1} \leftarrow{ }^{1} A_{1}$ transition, and band $A$ is assigned to the ${ }^{2} A_{1}\left({ }^{2} \Pi_{3}\right) \leftarrow{ }^{1} A_{1}$ transition. It's not clear that the origins chosen for the simulations are definitive, particulariy for the very extended progression in band $A$, but an approximate value for the Renner-Teller relaxation can be determined from the energy interval between the twc bands, which is approximately $160 \mathrm{~cm}^{-1}$. This is substantially less than the predicted value, 800 to $1600 \mathrm{~cm}^{-1}$ [Ref. (3)] suggesting that the bond angle of the ${ }^{2} \mathrm{~B}_{1}$ state may be wider than predicted, which makes it even more feasible that a $150^{\circ}$ anion could overlap with both bent and linear components. Moreover, it is possible that the ${ }^{t} \mathrm{~A}_{1}$ component is only nearly linear or quas,-linear, further facilitating Franck-Condon overtap with a bent anion.

Finally, from Weltner's work on GaAs diatomics and mixed triatomics trapped in raregas matrices, a frequency for the antisymmetric stretch of the ground state of $\mathrm{Ga}_{2} \mathrm{As}$ was determined to be 204.0 to $205.4 \mathrm{~cm}^{-1}$, depending on the $\mathrm{Ga}$ isotopic arrangement. ${ }^{19}$ A weaker line at $160 \mathrm{~cm}^{-1}$ was noted as a possible candidate for $v_{1}$. The higher $v_{3}$ frequency is consistent 
with the wide-angle $\mathrm{C}_{2 v}$ molecule, and the $160 \mathrm{~cm}^{-1}$ suggested for $v_{1}$ is exactly what we independently determined for the $v_{1}$ frequency in our simulation of band $X$. This further supports ous assignment of band $X$ to the bent $\leftarrow$ bent transition, and since band $A$ has the more extended progression in the bend, the possibiliky that it is a linear $\leftarrow$ linear transition seems less likely, and the linear $\leftarrow$ bent transition assignment seems further reinforced.

Comparison between the $\mathrm{Ga}_{2} \mathrm{As}^{-}$and $\ln _{2} \mathrm{P}^{-}$ZEKE spectra. The striking similarity between the band structure in the PES of $\mathrm{Ga}_{2} \mathrm{As}^{-}$and $\ln _{2} \mathrm{P}^{-}$suggests that these two molecules have the same overall electronic structure in both the anions and neutrals. Because both components of the Renner-Tcller pair are cbserved in the $\mathrm{Ga}_{2} \mathrm{As}^{*}$ spectrum, the anion must have the ${ }^{1} A_{1}$ electronic orbital configuration. Any triplet anion could not access both components via a one-electron transition. This then implies that the $\ln _{2} \mathrm{P}$ ground state has the ${ }^{1} \mathrm{~A}_{1}$ configuration. However, the observation of the two Renner-Teller components in the $\mathrm{Ga}_{2} \mathrm{As}^{-}$ spectrum contrasts sharply with our only observing the bent component in the $\ln _{2} \mathrm{P} \cdot$ spectrum. This suggests that the ground anion state of $\mathrm{Ga}_{2} \mathrm{As}^{-}$is more nearly linear than the ground anion state of $\mathrm{In}_{2} \mathrm{P}$ - While it is not immediately obvious why this is the case, we can speculate on the differences in bonding between the two species based on a few observations.

First, the $v_{2}$ frequencies in both $\ln _{2} P$ and $\mathrm{Ga}_{2} A$ are nearly identical, despite the fact that the reduced mass of $\mathrm{Ga}_{2} \mathrm{As}$ is much higher than $\ln _{2} P$. An economical CISD calculation performed using the Gaussian 92 software package gave a $25 \mathrm{cmi}^{-1}$ bend frequency for the ${ }^{2} \mathrm{~B}_{1}$ ground state of $\ln _{2} P$ and a reduced mass of 45 amu, 30 and since the calculated frequency is low, either the force constant was underestimated, or the reduced mass was overestimated for both). On the other hand, the reduced mass of $\mathrm{Ga}_{2} \mathrm{As}$ for any mode will be over 69 amu. Therefore, the bend potential for $\mathrm{Ga}_{2} A s$ must actually be stiffer than for $\ln _{2} P$, and the $G a$ atoms, in spite of their lower mass, are not necessarily moving with greater amplitude than the In atoms. Tre stiffer bending potential in $\mathrm{Ga}_{2} \mathrm{As}$ may be due to the better overlap between the p-orbitals of all three atoms in the molecule; for $\ln _{2} P$, the end p-orbitals are much larger than the p-orbital of 
the phosphorous atom. The larger p-orbitals in the In atoms may aiso lend to further RennerTeller stabilization since the closer the In atoms are, the better the mutual p-orbital overlap. The In $\mathrm{P}$ molecule does not have to bend as much in order to get some overlap between the endatom atomic orbitals as does $\mathrm{Ga}_{2} \mathrm{As}$. The In-In bond distance is calculated to be $0.3 \AA$ longer than the Ga-Ga bond distance, while the bulk in-P bond distance is only $0.09 \dot{A}$ longer than the GaAs buik bond distance. Therefore, it is possible that the electronic character of $\ln _{2} P$ is more sensitive to the bend (i.e., stronger Renner-Teller interaction, which subsequently implies a smaller bond angle in the bent component) than does $\mathrm{Ga}_{2} \mathrm{As}$.

Another interesting difference between the two species that can be extracted from the spectra is that the $\ln _{2} P^{-} v_{2}$ frequency was found to be much higher than the neutral $v_{2}$ frequency, while for $\mathrm{Ga}_{2} \mathrm{As}$, the anion and neutral frequencies are comparable. All of this points to the $1 b_{1}$ orbital having more In-In bonding character in $\ln _{2} P$ than $G a-G a$ bonding in $G_{a_{2}} A s$, which is the result of the stronger Renner-Teller interaction for the indium phosphide. Unfortunately, the anion frequencies determined from the spectra are not definitive; they are based to a larye extent on progr.:sion profiles which could be affected by other things souch as mode coupling. Hence, the above arguments should be considered only speculative, and naturally, some higher-level frequency calculations are desired for these complex species.

\section{v. Conclusion}

The negative ion photodetachment spectra of small gallium arsenide clusters presented in this paper exhibit the electronic complexity characteristic of small semiconductor ciusters. The PES of mass-resolved $\mathrm{Ga}_{x} A s_{y}{ }^{-}(x=1,2 ; y=1,2,3)$ obtained using $4.66 \mathrm{eV}$ photon energy access at least two electronic bands for each cluster, and in the case of $\mathrm{Ga}_{2} \mathrm{As}^{-}$, the higherresolution ZEKE spectrum reveals two partially overlapping electronic transitions where only one is observed in the PES. The PES of $\mathrm{GaAs}_{2}{ }^{-}$and $\mathrm{GaAs}_{3}{ }^{-}$show considerably more structure than 
their $\ln \mathrm{P}_{\mathrm{y}}-$ counterparts, suggesting that the As atoms dominate the bonding in these As-rich species. The $\mathrm{Ga}_{2} \mathrm{As}_{\mathrm{y}}{ }^{*}$ spectra more closely resemble the analogous $\ln _{2} \mathrm{P}_{\mathrm{y}}$ - clusters, and the similar electronic structures suggest more equivalent bonding between all of the atoms in the cluster. The $\mathrm{Ga}_{2} \mathrm{As}_{2}{ }^{-}$spectrum is even similar to the PES of isoelectronic $\mathrm{Si}_{4}{ }^{-}$, further supporting this assessment.

The higher-resolution PES of $\mathrm{GaAs}^{\star}$ shows partially-resolved electronic transitions to the low-lying triplet states of neutral GaAs as well as well-resolved transitions to the higher-lying singlet states. Analysis of the singlet band, which lies approximately $0.55 \mathrm{eV}$ higher in energy than the triplet band, indicates that the ${ }^{2} \Pi(3 / 2)$ anion state lies $80 \mathrm{~cm}^{-1}$ higher than the ${ }^{2} \Sigma^{+}$ ground anion state. Also, the ${ }^{1} \Sigma^{+}$excited neutral state lies $1306 \mathrm{~cm}^{-1}$ higher than the ${ }^{1} \Pi$ state, which is the lowest-lying of the reutral states.

The ZEKE spectrum of $\mathrm{Ga}_{2} A s^{-}$shows two close-lying neutral transitions that appear to be transitions from the ${ }^{1} A_{1}$ anion state to the bent ${ }^{2} B_{1}$ and linear ${ }^{2} A_{1}\left({ }^{2} \Pi_{u}\right)$ neutral states, which are the two components of a Renrisi-Teller pair. The two states are approximately $160 \mathrm{~cm}^{-1}$ apart, suggesting that the ${ }^{2} \mathrm{~B}_{1}$ component has a very broad bend angle. Two vibrational modes are active in both bands. For the bent component, the $v_{2}$ bend is $50 \pm 2 \mathrm{~cm}^{-1}$, and the $v_{1}$ symmetric stretch is determined to be approximately $160 \mathrm{~cm}^{-1}$ from spectral simulations. This is identical to a line observed in GaAs clusters trapped in rare gas matrices that could be attributed to $v_{1}$ of the $\mathrm{Ga}_{2}$ As ground state [Ref. (19)]. In the transition to the linear component, the progression in the $47.0 \pm 0.7 \mathrm{~cm}^{-1}$ bend mode is much more extended, and the stretch frequency is $171 \mathrm{~cm}^{-1}$. The anion stretch is determined from $z$ hot band transitions to be 259 $\mathrm{cm}^{-1}$. The ZEKE spectrum of $\mathrm{Ga}_{2} A s^{-}$differs from the specirum of $\ln \mathrm{n}_{2} \mathrm{P}$ in that the $\ln _{2} \mathrm{P}^{-}$ spectrum only exhibits one distinct electronic transition. This is counterintuitive based on the remarkable similarity between the respective PES. This suggests that while the electronic structures are similar between these species, the bonding between the constituent atoms, the 
group III atoms in particular, is different. This may be due to the differences in the atomic sizes or the disparate polarities along the III-V bonds.

\section{Acknowledgments}

This work is supported under NSF grant no, DMR-9201159. 
1 J. Faist, F. Capasso, D. L. Sivco, C. Sirtori, A. L. Hutchinson, and A. Y. Cho, Science 264, 553 (1994).

$2 \mathrm{Ga}_{x} A s_{x}$ M. A. Al-Laham and K. Raghavachari, Chem. Phys. Lett. 187, 13 (1991); $A I_{x} P_{x}$ and $M g_{x} S_{x}$ M. A. Al-Laham, G. W. Trucks, and K. Paghavachari, J. Chem. Phys. 96.1137 (1992).

${ }^{3}$ GaAs and insb mixed triatomics: K. K. Das and K. Balasubramanian, J. Chem. Phys. 94, 6620 (1991).

${ }^{4} G a_{x} 4 s_{y}$ U. Meier, S. D. Peyerimhoff, and F. Grein, Chem. Phys. 150, 331 (1991).

$5 \mathrm{Ga}_{x} A s_{y}:$ L. Lou, L. Wang, L. P. F. Chibante, R. T. Laaksonen, P. Nordlander, and R. E. Smailey, J. Chem. Phys. 94, 8015 (1991); L. Lou, P. Nordlander, and R. E. Smalley, J. Chem. Phys. 97, 1858 (1992).

${ }^{6}$ R. M. Graves and G. E. Scuseria, J. Chem. Phys. 95, 6602 (1991).

${ }^{7}$ E. C. Honea, J. S. Kraus, J. E. Bower, and M. F. Jarrold, Z. Phys. D 26, 141 (1993); E. C. Honea, A. Ogura, C. A. Murray, K. Raghavachari, W. O. Sprenger, M. F. Jarrold, and W. L. Brown, Nature 366, 42 (1993).

${ }^{B}$ C. Xu, E. deBeer, D. W. Amold, C. C. Amold, and D. M. Neumark, J. Chem. Phys. 100, 5406 (1994).

${ }^{9}$ C. C. Arnold and D. M. Neumark, Canı. J. Phys. (1994) in press.

10 T. N. Kitsopoulos, C. J. Chick, Y. Zhao, and D. M. Neumark, J. Chem. Phys. 95, 5479 (1991);

C. C. Arnold, T. N. Kitsopoulos, and D. M. Neumark, J. Chem. Phys. 99, 766 (1993).

11 T. N. Kitsopoulos, C. J. Chick, A. Weaver, and D. M. Neumark, J. Chem. Phys. 93, 6108 (1990); C. C. Amold and D. M. Neumark, J. Chem. Phys. 99, 3353 (1993); C. C. Arnold and D. M. Neumark, J. Chem. Phys. 100, 1797 (1994). 
12 G. R. Surton, C. Xu, and D. M. Neumark, (in preparation); C. C. Amold, C. Xu, G. R. Burton, and D. M. Neumark (in preparation).

${ }^{13}$ E. P. Wigner, Phys. Rev. 73, 1002 (1948).

14 K. J. Reed, A. H. Zimmerman, H. C. Andersen, and J. I. Brauman, J. Chem. Phys. 64, 1368 (1976).

15 K. D. Kolenbrander and M. L. Mandich, J. Chem. Phys. 92, 4659 (1990); K. -D. Rinnen, K. D. Kolenbrander, A. M. DeSantolo, and M. L. Mandich, J. Chem. Phys. 96, 4088 (1992); M. L. Mandich and K. D. Rinnen, Z. Phys. D 26, 147 (1993).

${ }^{16}$ C. Jin, K. J. Taylor, J. Conceicao, and R. E. Smalley, Chem. Phys. Lett. 175, 17 (1990).

17 G. W. Lemire, G. A. Bishea, S. A. Heidecke, and M. D. Morse, J. Chem. Phys. 92, 121 (1990).

18 S. Li, R. J. Van Zee, and W. Weltner, Jr., J. Phys. Chem. 98, 2275 (1994).

19 S. Li, R. J. Van Zee, and W. Weltner, Jr., J. Phys. Chem. 97, 11393 (1993).

20 U. Meier, S. D. Peyerimhoff, P. J. Bruna, and F. Grein, J. Mol. Spec. 134, 259 (1989).

21 K. Balasubramanian, J. Mol. Spec. 139, 405 (1990).

22 T. G. Dietz, M. A. Duncan, D. E. Powers, and R. E. Smalley, J. Chem. Phys. 74, 6511 (1981).

${ }^{23}$ R. B. Metz, A. Weaver, S. E. Bradforth, T. N. Kitsopoulos, and D. M. Neumark, J. Phys. Chem. 94, 1377 (1990).

${ }^{24}$ D. Proch and T. Trickl, Rev. Sci. Instrum. 60, 713 (1989). 
${ }^{25}$ T. N. Kitsopoulos, I. M. Waller, J. G. Loeser, and D. M. Neumark, Chem. Phys. Lett. 159, 300 (1989); C. C. Amold, Y. Zhao, T. N. Kitsopoulos, and D. M. Neumark, J. Chem. Phys. 97, 6121 (1992).

${ }^{26}$ K. Balasubramanian, J. Phys. Chem. 94, 7764 (1990).

${ }^{27}$ H. Hotop and W. C. Lineberger, J. Phys. Chem. Ref. Data 14, 73 1 (1985).

$28 \mathrm{~J}$. Berkowitz, Photoabsorption, Photoionization, and Photoelectron Spectroscopy (Academic, New York, 1979).

${ }^{29}$ C. M. Rohlfing and K. Raghavachari, J. Chem. Phys. 96, 2214 (1992).

30 The LANLDZ effective core potentia! basis set was used; Gaussian 92, Revision E.2, M. J. Frisch, G. W. Trucks, M. Head-Gordon, P. M. W. Gill, M. W. Wong, J. B. Foresman, B. G. Johnson, H. B. Schlegel, M. A. Robb, E. S. Replogle, R. Gomperts, J. L. Andres, K. Raghavachari, J. S. Binkley, C. Gonzalez, R. L. Martin, D. J. Fox, D. J. Detrees, J. Baker, J. J. P. Stewart, and J. A. Pople, Gaussian, Inc., Pittsburgh, PA, 1992. 
Table (10.1) Adiabatic electron affinities and term energies of the low-lying electronic states of $\mathrm{Ga}_{x} \mathrm{As}_{y}$ clusters $(x=1,2 ; y=1,2,3)$. For $y>t$, values are determined from the $4.657 \mathrm{eV}$ PES and are considered good to $\pm 0.05 \mathrm{eV}$. For $\mathrm{Ga}_{2} \mathrm{As}$, the EA. is determined from the origin of the ZEKE spectrum, and the $T_{e}(A)$ is from the $4.657 \mathrm{eV}$ PES. The EA and $T_{e}$ 's for GaAs are determined from the $2.98 \mathrm{eV} P E S$.

Cluster Energetics

(ev)

GaAs

$\mathrm{Ga}$ As $\quad E A=2.452^{*}$
Cluster

Energetics

(eV)

GaAs?

$E A=1.83$

$T_{A}(A)=0.74$

$T_{A}(B)=2.01$

$\mathrm{Ga}_{2} \mathrm{As}, \quad E A=1.87$

$T_{A}(A)=0.70$

$T_{A}(B)=1.59$

$\mathrm{T}_{a}(C)=2.41$
Cluster Energetics (eV)

$\mathrm{GaAs}_{\mathbf{3}} \quad \mathrm{EA}=\mathbf{1 . 9 5 1}$

$T_{A}(A)=0.43$

$T_{A}(B)=1.06$

$T_{A}(C)=1.87$

$\mathrm{Ga}_{2} \mathrm{As}_{\mathfrak{3}} \quad E A=2.66$

$T_{A}(A)=0.38$ 
Table (10.2) Peak positions and relative energies for the PES of GaAs- obtained using 2.98 eV photon energy.

\begin{tabular}{|c|c|c|c|}
\hline Peak & $\begin{array}{l}\text { Peak Position, eKE } \\
(\mathrm{eV})\end{array}$ & $\begin{array}{l}\text { Relative energy } \\
\left(\mathrm{cm}^{-1}\right)\end{array}$ & Tentative Assignment \\
\hline$c$ & 1.039 & -460 & \\
\hline$b$ & $1.022^{\dagger}$ & -323 & \\
\hline a & $1.005^{*}$ & -186 & \\
\hline 1 & $0.982^{\dagger t}$ & 0 & \\
\hline 2 & 0.962 & 161 & \\
\hline 3 & $0.955^{\dagger}$ & 218 & \\
\hline 4 & $0.929^{*}$ & 427 & \\
\hline 5 & 0.896 & 694 & \\
\hline 6 & $0.871^{*}$ & 895 & \\
\hline 7 & 0.861 & 976 & \\
\hline 8 & $0.838^{*}$ & $116 i$ & \\
\hline 9 & $0.806^{*}$ & 1419 & \\
\hline 10 & $0.797^{*}$ & 1492 & \\
\hline 11 & 0.766 & 1742 & \\
\hline 12 & $0.751^{*}$ & 1863 & \\
\hline 13 & $0.736^{*}$ & 1984 & \\
\hline 14 & $0.708^{*}$ & 2210 & \\
\hline 15 & $0.676^{*}$ & 2468 & \\
\hline 16 & $0.579 t$ & 3250 & \\
\hline & -_.........- & m.n-m.m & 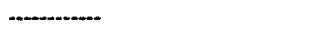 \\
\hline 17 & $0.447^{\dagger}$ & 4315 & ${ }^{1} \Pi\left(v^{\prime}=0\right) \leftarrow{ }^{2} I(3 / 2)\left(v^{\prime \prime}=1\right)$ \\
\hline 18 & $0.434^{\dagger}$ & 4420 & \\
\hline 19 & $0.416^{\dagger}$ & 4565 & ${ }^{1} \Pi\left(v^{\prime}=0\right) \leftarrow{ }^{2} \Pi(3 / 2)\left(v^{\prime \prime}=0\right)$ \\
\hline 20 & 0.406 & 4645 & ${ }^{1} \Pi\left(v^{\prime}=0\right) \leftarrow{ }^{2} \Sigma^{+}\left(v^{\prime \prime}=0\right)$ \\
\hline 21 & 0.381 & 4847 & ${ }^{1} \Pi\left(v^{\prime}=1\right) \leftarrow{ }^{2} \Pi(3 / 2)\left(v^{\prime \prime}=0\right)$ \\
\hline 22 & $0.368^{*}$ & 4952 & ${ }^{1} \Pi\left(v^{\prime}=1\right) \leftarrow-2 \Sigma^{+}\left(v^{\prime \prime}=0\right)$ \\
\hline 23 & $0.345^{\dagger}$ & 5137 & ${ }^{1} \Pi\left(v^{\prime}=2\right) \leftarrow{ }^{2} \Pi(3 / 2)\left(v^{\prime \prime}=0\right)$ \\
\hline 24 & $0.335^{*}$ & 5218 & ${ }^{1} \Pi\left(v^{\prime}=2\right) \leftarrow 2 \Sigma^{+}\left(v^{\prime}=0\right)$ \\
\hline 25 & $0.305^{*}$ & 5460 & \\
\hline 26 & $0.292^{\dagger}$ & 5565 & ${ }^{1} \Pi\left(v^{\prime}=3\right) \leftarrow{ }^{2} \Sigma^{+}\left(v^{\prime \prime}=0\right)$ \\
\hline 27 & 0.277 & 5686 & \\
\hline 28 & $0.254^{\dagger}$ & 5871 & ${ }^{1} \Sigma^{+}\left(v^{\prime}=0\right) \leftarrow^{2} \Sigma^{+}\left(v^{\prime \prime}=0\right)$ \\
\hline 29 & $0.219^{\dagger}$ & 6154 & \\
\hline
\end{tabular}


Table (10.3) Peak positions and relative energies for the ZEKE spectrum of $\mathrm{Ga}_{2} \mathrm{As}^{-}$.

\begin{tabular}{|c|c|c|c|}
\hline Peak & $\begin{array}{l}\text { Position } \\
\left(\mathrm{cm}^{-1}\right)\end{array}$ & $\begin{array}{l}\text { Relative } \\
\text { Energy } \\
\left(\mathrm{cm}^{-1}\right)\end{array}$ & $\begin{array}{l}\text { Tentative } \\
\text { Assignment }\end{array}$ \\
\hline$x_{p}^{\prime}(0)$ & 19518 & -259 & $x(1,0)$ \\
\hline$x_{0}^{\prime}(1)$ & 19551 & -226 & $X\left(1,2_{0}^{\prime}\right)$ \\
\hline$x_{2}^{\prime}(2)$ & $\begin{array}{l}19569 \\
19729\end{array}$ & $\begin{array}{l}-208 \\
-48\end{array}$ & $\begin{array}{l}X\left(1,2_{0}^{2}\right) \\
X\left(2_{1}^{0}\right)\end{array}$ \\
\hline$x_{2}(0)$ & $19 \pi 7$ & 0 & Band $X$ origin \\
\hline$x_{7}(1)$ & 19827 & 50 & $X\left(2_{0}^{\prime}\right)$ \\
\hline$x_{2}^{\prime}(2)$ & $\begin{array}{l}19876 \\
19897\end{array}$ & $\begin{array}{l}96 \\
120\end{array}$ & $\begin{array}{l}x\left(2_{0}^{2}\right) \\
A\left(2^{0}\right)\end{array}$ \\
\hline$x_{2}(3)$ & 19922 & 145 & $x\left(2_{0}^{3}\right)$ \\
\hline$x_{1}(0)$ & 19938 & 101 & $X\left(1_{0}^{1}\right)$ \\
\hline$A_{0}(0)$ & 19950 & 173 & Band $\mathbf{A}$ origin \\
\hline$x_{12}(1)$ & 19975 & 198 & $x\left(10_{0}^{\prime}\right)$ \\
\hline$A_{2}(1)$ & 19988 & 211 & $A\left(2_{0}^{3}\right)$ \\
\hline$A_{0}(2)$ & 20039 & 262 & $A\left(2_{0}^{2}\right)$ \\
\hline & 20071 & 294 & $A\left(t_{0}^{1} 2_{1}^{\circ}\right)$ \\
\hline$A_{2}(3)$ & 20085 & 308 & $A\left(2_{0}^{3}\right)$ \\
\hline$A_{1,}(0)$ & 20114 & 337 & $A\left(1_{0}^{1}\right)$ \\
\hline$A_{0}(4)$ & 20131 & 354 & $A\left(2_{0}^{4}\right)$ \\
\hline$A_{1}(1)$ & 20163 & 386 & $A\left(1_{0}^{1} 2_{0}^{1}\right)$ \\
\hline$A_{2}(5)$ & 20178 & 401 & $A\left(2_{0}^{5}\right)$ \\
\hline$A_{1},(2)$ & 20209 & 432 & $A\left(12_{0}^{2}\right)$ \\
\hline$A_{2}(6)$ & 20229 & 452 & $A\left(2_{0}^{6}\right)$ \\
\hline$A_{1}(3),\left[A_{2}(7)\right]$ & 20257 [ [20 279] & $480[502]$ & $A\left(1_{0}^{1} 2_{0}^{3}\right),\left[\left(2_{0}^{7}\right)\right]$ \\
\hline$A_{1}(4)$ & 20306 & 539 & $A\left(1_{0}^{\prime} 2_{0}^{d}\right),\left(1_{0}^{2}\right)$ \\
\hline$A_{12}(5)$ & 20356 & 579 & $A\left(12_{0}^{5}\right),\left(1_{0}^{2} 2_{0}^{\prime}\right)$ \\
\hline$A_{1},(6)$ & 20402 & 625 & $A\left(1_{0}^{1} 2_{0}^{6}\right),\left(1_{0}^{2} 2_{0}^{2}\right)$ \\
\hline$A_{10}(7)$ & 20450 & 673 & $A\left(10_{0}^{1} 2_{0}^{7}\right),\left(1_{0}^{2} 2_{0}^{3}\right)$ \\
\hline$A_{1},(8)$ & 20501 & 724 & $A\left(10_{0}^{1} 2^{8}\right),\left(10_{0}^{2} 2_{0}^{4}\right)$ \\
\hline
\end{tabular}


Figure (10.1) Anion PES of small $\mathrm{Ga}_{x} \mathrm{As}_{y}$ clusters cbtained using $4.66 \mathrm{eV}$ photon energy.

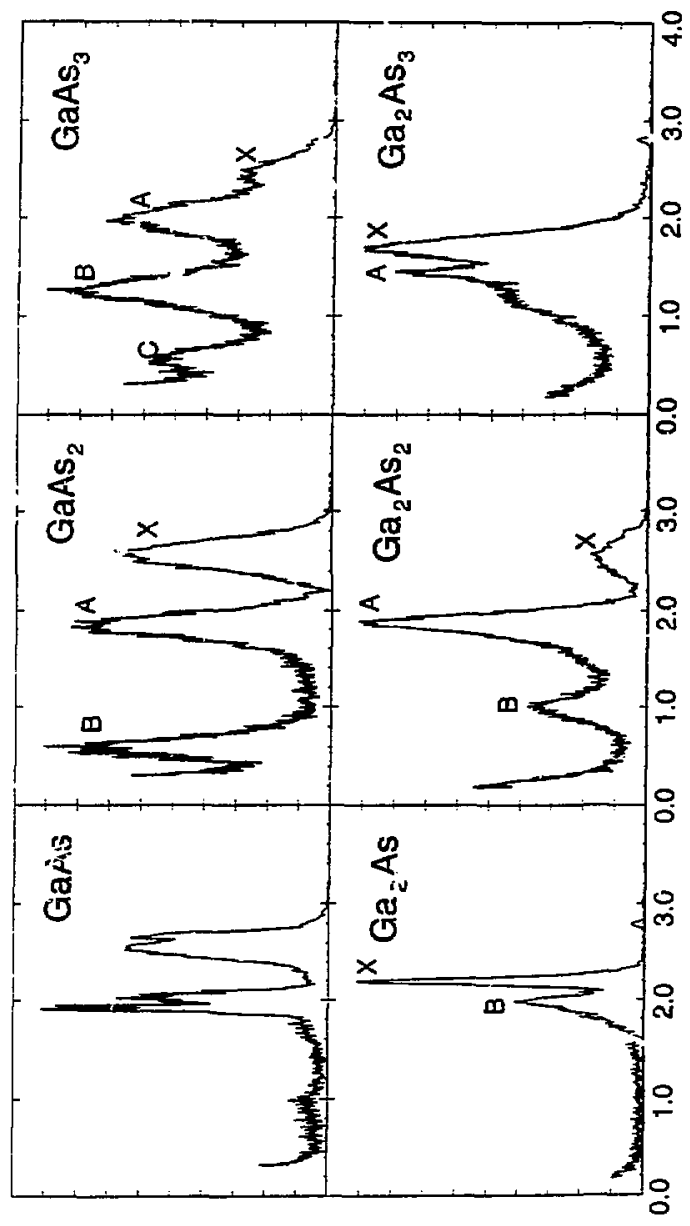


Figure (10.2) Higher resolution PES of GaAs obtained at three different laser polarizations and a photon energy of $2.98 \mathrm{eV}$.

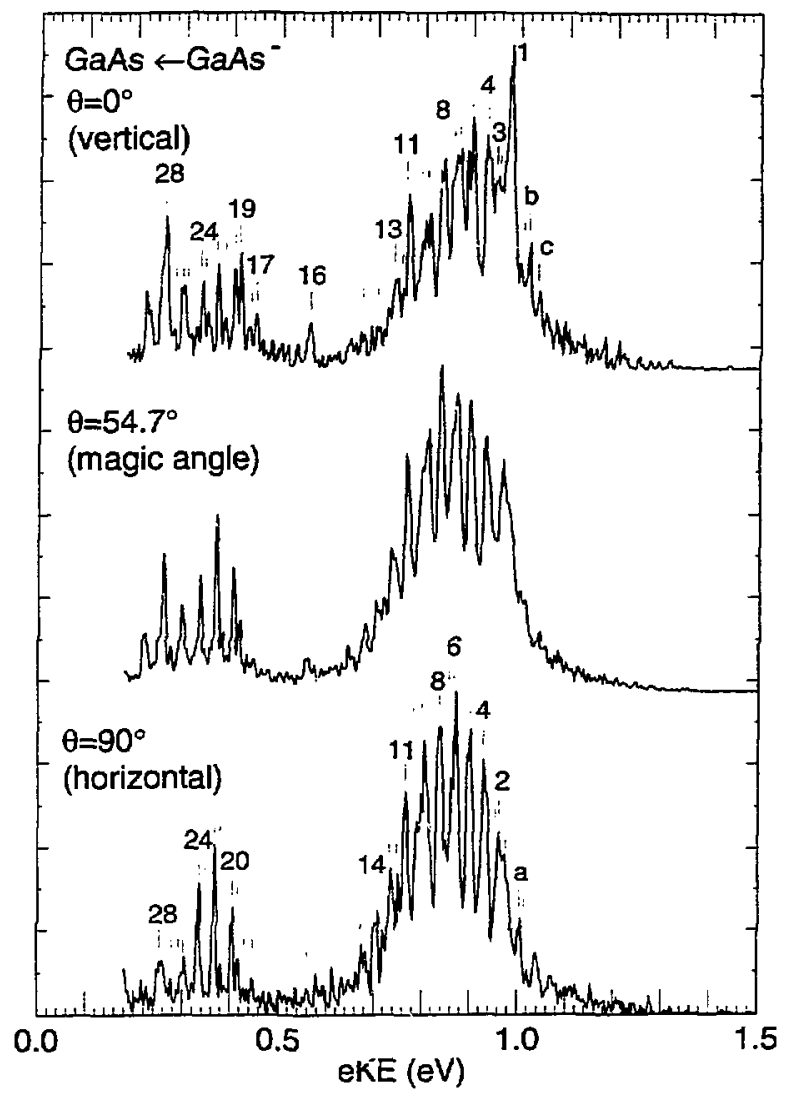


Figure (10.3) Companison of 4.66 eV PES (dashed line) and ZEKE spectra (solid line) of $\mathrm{Ga}_{2} \mathrm{As}^{-}$(upper traces) and isoelectronic $\ln _{2} \mathrm{P}^{-}$(lower traces).

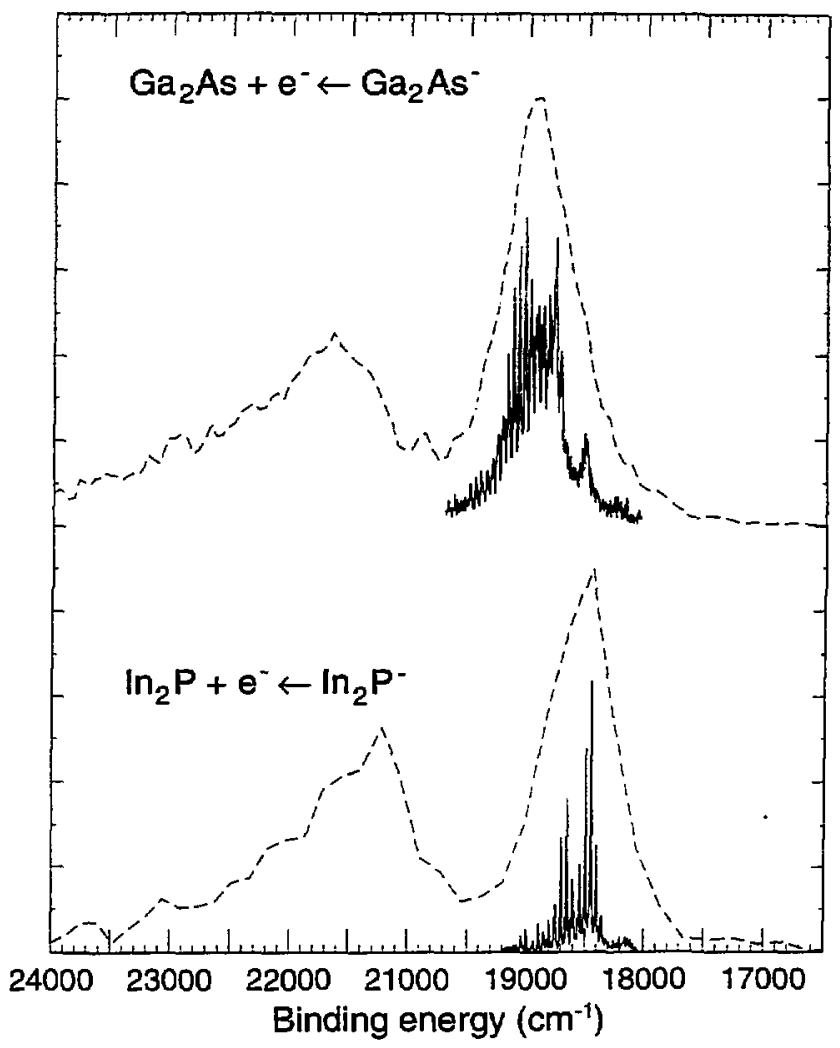


Figure (10.4) ZEKE spectnum of $\mathrm{Ga}_{2} \mathrm{As}$ :

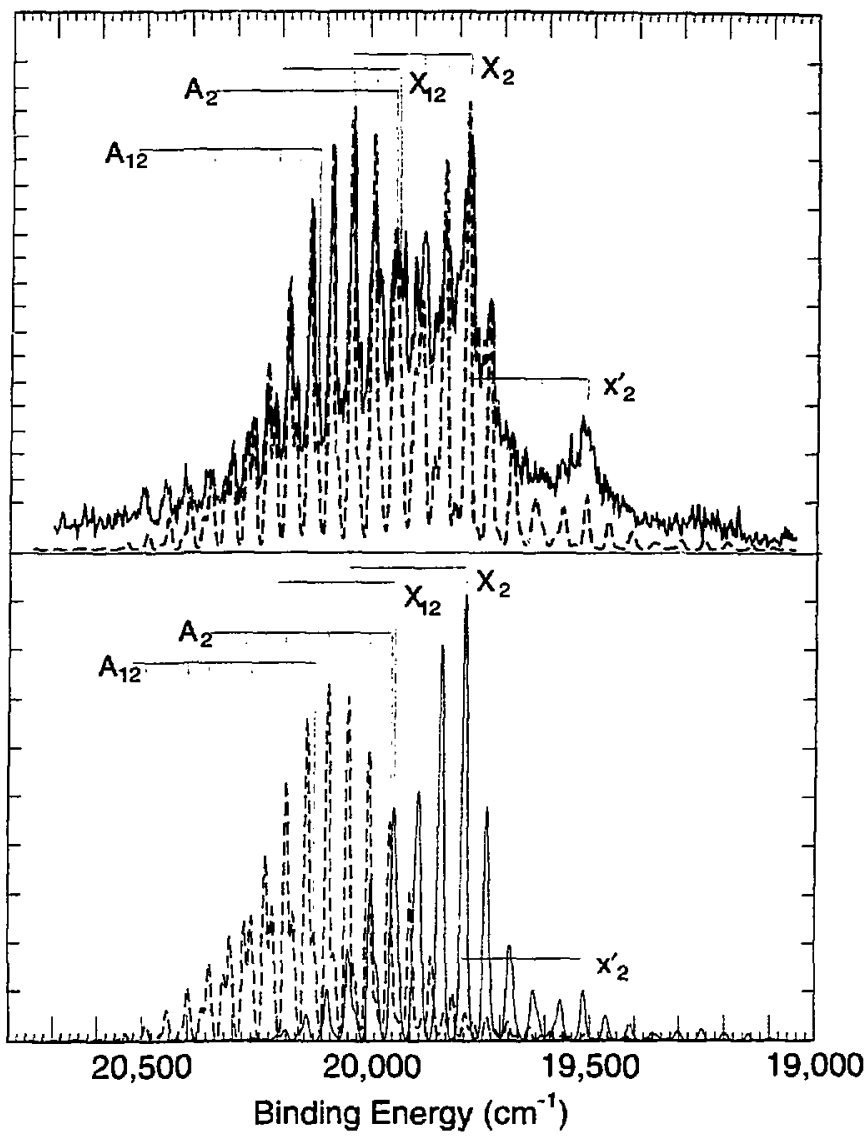


Figure (10.5) One-electron iransitions between the two low-lying electronic states of GaAs" and the five lowest-lying electronic states of GaAs.

$\mathrm{GaAs}_{2}$

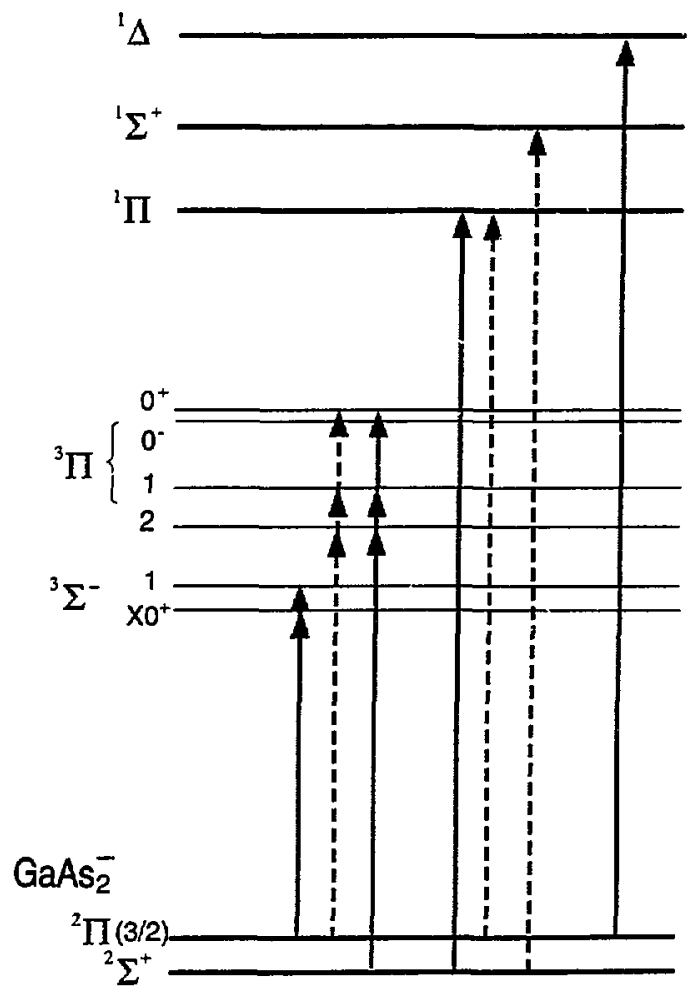




\section{Chapter 11. The power of negative ion ZEKE Spectroscopy; an aside on the $\mathrm{I} \cdot \mathrm{CH}_{3} \mathrm{I} \mathrm{S}_{\mathrm{N}} 2$ reaction complex}

\section{Abstract}

The negative ion zero electron kinetic energy (ZEKE) spectrum of $\mathrm{I}^{-} \cdot \mathrm{CH}_{3} \mathrm{l}$ is presented and discussed. In addition to the high frequency vibrational structure previously seen in the photoelectron spectrum (PES) of I- $\cdot \mathrm{CH}_{3}$ l, the ZEKE spectrum reveals low frequency vibrational structure due to excita! ion in two van der Waals modes in the ground $(X)$ statt of the neutral 1. $\mathrm{CH}_{3}$ I complex. Of the two other excited states of the neutral complex, the I state is not distinctly observed, and no vibrational structure due to van der Waals motion is observed for the ll state. 


\section{Introduction}

The dynamics of the intermediates of an $X^{-}+\mathrm{CH}_{3} Y \rightarrow \mathrm{CH}_{3} X+Y^{-}$gas phase $\mathrm{S}_{\mathrm{N}^{2}} 2$ reaction have recently been the focus of considerable investigation, both theoretically and experimentally. $1,2,3,4,5,6,7,8,9$ The ion-dipole stabilized $\mathrm{I}^{-} \cdot \mathrm{CH}_{3}$ l complex, an intermediate in the $\mathrm{I}^{-}$ $+\mathrm{CH}_{3} \mathrm{l}$ identity reaction, has been of particular interest. Recently, Cyr et al. have combined photoelectron spectroscopy (PES) ${ }^{1}$ with photofragmentation studies ${ }^{2}$ to show that chargetransfer excited states of $\mathrm{I}^{-} \cdot \mathrm{CH}_{3} \mathrm{l}$, which are believed to be important in the reaction, ${ }^{3}$ lie near the detachment continuum of the anion complex. The vibrational structure obsened in the PES of $1^{-}$ $\mathrm{CH}_{3} \mathrm{l}$ suggests that the $\mathrm{C}-\mathrm{I}$ bond in the nominally neutral methyl iodide moiety has an elongated C-I bond, which is likely the result of the ground ion-dipole state mixing with the charge transfer state. ${ }^{1}$ This bond elongation has also been predicted in recent calculations by Truhlar. ${ }^{4}$

The experiments Cyr et al. are quite striking in that photofragmentation of $\mathrm{I}^{-} \cdot \mathrm{CH}_{3} \mathrm{l}$ into an anion and neutral fragment is only observed in the vicinity of the threshold for photodetachment. This situation represents an intra-cluster analogue of the phenomenon recently reported by Chupka and co-workers in which $\mathrm{F}$ is observed in the multiphoton ionization of $\mathrm{CH}_{3} \mathrm{l}$ by dissociative attac:3ment of low energy photoelectrons onto $\mathrm{CH}_{3} l^{10}$ in order to bett.ar understand the competition between photofragmentation and photodetachment, it is useful to characterize the neutral $\mid \cdot \mathrm{CH}_{3}$ l complex formed by photodelachment of the anion to a greater extent than was possible from the photoelectron spectrum alone. In this paper, we present the higher resolution negative ion zero electron kinetic energy, or ZEKE, spectrum of $1^{-} \cdot \mathrm{CH}_{3} \mathrm{l}$ in which we observe that the neutral complex is bound sufficiently strongly to support several van der Waals- type vibrations, at least in the ground spin-orbit state. Van der Waals vibrational structure has also been observed in previously obtained negative ion ZEKE spectra of the similar but simpler ionneutral complexes, namely $\mathrm{I}^{-} \cdot \mathrm{CO}_{2}{ }^{11}$ and several rare-gas halides. ${ }^{12}$ The implications of our 
results on the $\mathrm{r} \cdot \mathrm{CH}_{3} \mathrm{l}$ complex will be discussed in the context of the PES and photofragmentation studies.

\section{li. Experimental}

The anion ZEKE apparatus has been described in detail previously, ${ }^{13}$ but the basic operation is as follows. The $\mathrm{I}^{-} \cdot \mathrm{CH}_{3} \mathrm{l}$ complexes are generated and cooled in a pulsed free jet intersected by a $1 \mathrm{keV}$ electron beam per the methods of Johnson and Lineberger ${ }^{14}$ and are mass-selected using time-of-flight. The $\mathrm{l}^{-} \cdot \mathrm{CH}_{3} \mathrm{l}$ complex is then photodetached using a tunable dye laser, and those electrons detached with nearly zero electron kinetic energy are collected as a function of detachment wavelength. This selective detection of low-kinetic energy electrons, based on methods developed by Müller-Dethlets et al., ${ }^{15}$ yields an energy resolution as good as $\pm 3 \mathrm{~cm}^{-1}$. The electron signal is normalized to laser power and ion current. The dyes used in the scans on $1-\mathrm{CH}_{3} \mathrm{I}$ are DMQ and Coumarin 540. The latter was doubled using a BBO I doubling crystal. The spectra were signal averaged for over 3600 shots per point.

\section{Results and Discussion}

The ZEKE spectrum and the previously obtained PES ${ }^{2}$ of $\mathrm{r} \cdot \mathrm{CH}_{3} \mathrm{l}$ are shown in Figure (11.1), where the top pariel shows the PES obtained using a detachment wavelength of $4.66 \mathrm{eV}$, the center panel shows the ZEKE spectrum of band $\mathbf{X}$ in the PES, and the lower panel shows the ZEKE spectrum of band II in the PES. Band $\mathbf{X}$ in both the ZEKE spectrum and the PES corresponds to the transition to $\left.\mid\left({ }^{2} \mathrm{P}_{3 / 2}\right) \cdot \mathrm{CH}_{3}\right)$ complex from the anion, and band II is the transition to the spin-orbit excited I $\left({ }^{2} \mathrm{P}_{1 / 2}\right) \cdot \mathrm{CH}_{3}$ l complex. The two bands are separated by 0.955 $\pm 0.020 \mathrm{eV}$ in the ZEKE spectrum, which is very close to the spin-orbit splitting in the bare neutral iodine atom, $0.943 \mathrm{eV}$. Also, compared to the spectrum of bare $\mathrm{I}^{-}$, these features are 
shifted to higher photon energy by $0.365 \pm 0.020 \mathrm{eV}$, which is fairly close to the enthalpy of formation for the ion dipole complex, $\Delta \mathrm{H}_{\mathrm{a}}=-0.39 \pm 0.01 \mathrm{eV} .^{16}$

Both the photoelectron and ZEKE spectra exhibit high-frequency vibrational structure associated with excitation of vibrations in the neutral methyl iodide moiety: nominally the $v_{3} \mathrm{C}-1$ stretch in $\mathrm{CH}_{3}$ l. The $\mathrm{v}_{3}$ frequency extracted from the position of this $3_{0}^{1}$ transition in the ZEKE spectrum is approximately $550 \pm 20 \mathrm{~cm}^{-1}$, which is very close to the $\mathrm{v}_{3}$ frequency of bare $\mathrm{CH}_{3} \mathrm{l}$, $533 \mathrm{~cm}^{-1} .17$ Howeve:, band $X$ in the ZEKE spectrum exhibits further (partially) resolved lowfreqtiancy vibrational structure with spacings on the order of tens of wave numbers. This structure, the spacing of which is consistent with motion along a van der Waals bond, shows that this partic.'ar state of the neutral-dipole $1 \cdot \mathrm{CH}_{3}$ l complex is bound. The noise level in the ZEKE spectrum of band II is such that no definite structure could be unambiguously discerned. Since the van der Waals well for the excited spin-orbit state is almost certainly shallower than that for the ground state, ${ }^{11,12}$ the resulting lower frequency vibrational structure may be too congested to be resolved.

While the quality of the spectrum does not warrant the extensive analysis that we have previously applied to other weakly bound systems, ${ }^{11}$ there is a definite pattern to the positions of most of the peaks in the origin band. In order to find the simplest possible picture of the vibrational structure, we have performed a simulation wisizin the Franck-Condon approximation, in which transitions between vibrational levels of the anion $\left(v_{n}{ }^{\prime}\right)$ and the neutral $\left(v_{n}\right)$ are assumed proportional to their Franck-Condon factors, $\left|\left\langle v_{n} \| v_{n}\right\rangle\right|^{2}$. Figure (11.2) shows a simulation (solid trace) superimposed onto the ZEKE data (dotted trace). The simulation includes two low-frequency modes of the neutral, and the neutral vibrational wave function was taken to be the product of a Morse oscillator wave function with $\omega_{e}=54.9 \mathrm{~cm}^{-1}, \omega_{e} \chi_{e}=0.4 \mathrm{~cm}^{-1}$ (mode A) and a harmonic osciliator with $\omega_{e}=33 \mathrm{~cm}^{-1}$ (mode B). For a reasonable fit, the anion geometry had to be displaced substantially and comparably along both vibrational coordinates of the neutral. Therefore, the majority of the structure in the simulation is the result of combination 
bands (e.g., $A_{0}^{x} B_{0}^{y}, x, y=1,2, \ldots$ ) in these two modes. The $A_{0}^{y} B_{0}^{1}$ progression in the simulation is indicated on the comb in Figure (11.2) to guide the eye.

There is likely to be some excitation of the low fraquency anion modes as well, but due to the overall congestion of the ZEKE spectrum, it is difficult to determine which structure is due to hot bands or sequence bands, making the choice of anion frequencies for the simulation somewhat arbitrary. However, we found that if the anion frequency corresponding to mode $A$ is chosen to be approximately $100 \mathrm{~cm}^{-1}$, the width of the simulateo progression is in fairly good agreement with the observed progression. Moreover, if the anion frequency of mode $B$ was made to be $53 \mathrm{~cm}^{-1}$ ( $20 \mathrm{~cm}^{-1}$ greater then the neutral frequency), the level of congestion due to hot bands, and thus the intensities of peaks in the fit were closer to the experimental peak intensities. The choice of larger frequencies in the anion relative to the neutral is consistent with the stronger 1 .... $\left(\mathrm{CH}_{3} \mathrm{l}\right)$ interaction in the ion-dipole complex. For the particular simulation shown in Figure (11.2), the origin was placed at $365.04 \mathrm{~nm}$, the anion wave function was displaced from the neutral wave function substantially and comparably along the coordinates of both the $33 \mathrm{~cm}^{-1}$ and the $55 \mathrm{~cm}^{-1}$ modes, and anion vibrational levels were thermally populated at $50 \mathrm{~K}$. It should be noted that even assuming zero vibrational temperature in the anion, most of the peak positions could be matched simply assuming a large activation in the two neutral frequencies. Including transitions from excited anion levels in the simulation serves primarily to fill in some of the intensity. ${ }^{18}$

Truhlar calculated the two lowest frequency modes of the anion to be $64 \mathrm{~cm}^{-1}$ and 66 $\mathrm{cm}^{-1}$; the $100 \mathrm{~cm}^{-1}$ anion frequency used in our simulations is substantially higher than either of these. Simulations using Truhlar's anion frequencies gave profiles much more extended than in the experimental spectrum. In particular, when either the $64 \mathrm{~cm}^{-1}$ or $66 \mathrm{~cm}^{-1}$ frequency modes were taken as the anion mode corresponding to the neutral $55 \mathrm{~cm}^{-1}$ mode, the peak of the extended progression could be approprately simulated, but the simulated structure would tail much further to both the red and blue of the progression maximum than what is observed. 
However, because our treatment of what is likely a very complicated system is so simplified, our anion frequencies cannot be considered definitive. On the other hand, the two neutral frequencies are better determined as the $y$ correspond to observed peak spacings.

We now consider the electronic states of the anion and neutral, and the observed vibrational structure in terms of these electronic states. The anion electronic structure is fairly straightton:ard: a closed-shell anion is bound to a closed-shell neutral molecule by both chargedipole and dipole-induced dipole interactions in addition to some charge-transfer between the $\mathrm{I}^{-}$ and $\mathrm{CH}_{3} l^{3}{ }^{3}$ The calculations by Truhiar suggest that the $1 \cdot \mathrm{CH}_{3} l$ complex has $\mathrm{C}_{3 v}$ symmetry, and the anion electronic state is best described as a ${ }^{1} A_{1}$ state. As mentioned above, Truhlar's vibrational frequency calculations found two low frequency modes involving $\mathrm{I}^{-}-\left(\mathrm{CH}_{3} \mathrm{l}\right)$ relative motion. The $66 \mathrm{~cm}^{-1}$ mode is the $v_{4}\left(a_{1}\right) l^{-}-C$ stretch and the $64 \mathrm{~cm}^{-1}$ frequency corresponds to $v_{8}$, a degenerate (e) rocking mode. The calculated anion frequencies are too close to suggest whether the neutral $a_{1}$ or $e$ van der Waals mode should have the higher frequency.

In contrast to the simple closed-shell/closed-shell interaction of the anion, the sinieraction between an open shell, aisisotropic neutral halogen and a closed-shell moiety is complicated by spin-orbit and orientation effects. These considerations have been discussed in our previous work on $\mathrm{I}^{-} \cdot \mathrm{CO}_{2}{ }^{11}$ and $\left.\mathrm{Rg} \cdot \mathrm{X}^{-}(\mathrm{Rg}=\mathrm{Ar}, \mathrm{Kr}, \mathrm{Xe} ; \mathrm{X}=\mathrm{I}, \mathrm{Br}, \mathrm{Cl})\right)^{1.19}$ Figure (11.3) shows a schematic correlation diagram with the three electronic states of the neutral resulting from this interaction. Approximating $1 \cdot \mathrm{CH}_{3} \mathrm{I}$ as a point dipole $\left(\mathrm{CH}_{3} \mathrm{l}\right)$ interacting with an iodine atom, the electrostatic interactions cause a splitting of the ${ }^{2} \mathrm{P}_{3 / 2}$ ground spin-orbit level of iodine into an $\Omega$ $=1 / 2 \times$ state, and an $\Omega=3 / 2$ I state, where $\Omega$ is the projection of the electronic orbital and spin angular momentum onto the $C-I$ axis. In the Hund's case (c) limit, the $X$ state is composed of $1 / 3 \Pi$ and $2 / 3 \Sigma$ character, and the I state has only $\Pi$ character. ${ }^{20}$ The ${ }^{2} P_{1 / 2}$ level is not split; the resulting $\Omega=1 / 2$ II state has $2 / 3 \Pi$ and $1 / 3 \Sigma$ character in the case (c) limit. The well-depth of the $X$ state is the deepest, with the smallest equilibrium internuclear $\left(r_{C-1}\right)$ separation, and the $I$ state is the shallowest of the three states with the largest equilibrium internuclear separation. 
From the extent of the structure in the $\mathrm{I}^{-} \cdot \mathrm{CH}_{3} \mathrm{I}$ ZEKE spectrum, we can determine a lower limit of the well depth of the $X$ state of $I \cdot \mathrm{CH}_{3} I$ to be $250 \mathrm{~cm}^{-1}$. The true well depth is likely to be considerably larger; the well-depth of the $1 \cdot \mathrm{CO}_{2} \mathrm{X}$ state was more rigorously determined to be $359 \mathrm{~cm}^{-1},{ }^{11}$ and the $\mid \cdot \mathrm{CH}_{3} l$ well-depth is likely to be greater than this due to the stronger iondipole interaction.

The open shell nature of the neutral is also important in considering the vibrational structure associated with the $X$ state. Our analysis shows that at least two van der Waals modes are active, but only one of these (the $v_{4}$ mode) is totally symmetric in the $C_{3 v}$ point group. A progression in the degenerate $v_{8}$ mode (with $e$ symmetry) would normally not be expected if both the anion and neutral have $C_{3 v}$ symmetry; although transitions in which $\Delta v$ is even are allowed by symmetry, an extended progression in this mode is highly unlikely based on FranckCondon factors alone. ${ }^{21}$ Since $\Pi$ states correlate to degenerate $E$ states in $C_{3 v}$ symmetry, the $X$ state (and the other two electronic states) of $1 \cdot \mathrm{CH}_{3}$ l should possess have some $\mathrm{E}$ character. These states can then undergo Jahn-Teller distortion, thereby activating the low-frequency degenerate vibrational mode. This effect is well known in $E+-A$ electronic transitions, ${ }^{22}$ but is less well-characterized for systems with large spin-orbit coupling such as $1 \cdot \mathrm{CH}_{3} \mathrm{l}$.

The ZEKE spectia of $1^{-} \cdot \mathrm{CO}_{2}$ and several of rare gas halides show transitions to the I state of the neutral lying quite close to the $X$ band; the $X-1$ splitting varies from $225 \mathrm{~cm}^{-1}$ for I. $\mathrm{CO}_{2}{ }^{11}$ to $30-40 \mathrm{~cm}^{-1}$ for rare gas halogen dimers sontaining Ar or $\mathrm{Kr} .^{12,19}$ There is indirect evidence for a close-lying I state In the $1^{-} \cdot \mathrm{CH}_{3}$ l photoelectron spectrum; the peak widths in band $\mathbf{X}$ vary with the polarization direction of the photodetachment laser, suggesting they may consist of overlapping electronic transitions. However, no distinct I band is observed in the ZEKE spectrum of $1 . \mathrm{CH}_{3}$. The likely reason for this is that the potential energy minimum for the van der Waals stretch in the neutral I state lies at a considerably larger intemuclear distance than the minimum in the corresponding anion potential. This means that a vertical transition from the anion accesses the repulsive wall of the ! state, and resulting broad and featureless band would 
be ve difficult to observe in the ZEKE spectrum. Two factors favor this explanation. First of all, the dissociation energy of $\mathrm{I}^{1} \cdot \mathrm{CH}_{3} \mathrm{I}$ is substantially higher than that of $\mathrm{I}^{-} \cdot \mathrm{CO}_{2}, 0.39 \mathrm{eV}$ vs. $0.212 \mathrm{eV}$. Secondly, comparing the neutral complexes, 'me expects the I atom to interact more strongly with $\mathrm{CH}_{3}$ l than with $\mathrm{CO}_{2}$ because of the pe:manent dipole moment in $\mathrm{CH}_{3}$ l. This should result in an increased splitting between the $X$ and $I$ states, along with a larger difference in the equilibrium intemuclear distances. Thus, sompared to ${ }^{-} \cdot \mathrm{CO}_{2}$, one expects a larger difference in the equilibrium internuclear distances between the anion and $I$ states for $1-\mathrm{CH}_{3} \mathrm{l}$ photodetachment. A preliminary analysis indicates that the $\mathrm{I}$ band is missing in the $\mathrm{XeBr}$ - ZEKE spectrum for the same reason. ${ }^{19}$

The above discussion offers an explanation of an interesting result observed by Cyr et al. ${ }^{2}$ They found that the fast neutral peak resulting from photodetachment of $1 \cdot \mathrm{CH}_{3} \mathrm{l}$ at $266 \mathrm{~nm}$ was somewhat broader than the parent ion peak, indicating the production of neutral $1+\mathrm{CH}_{3} \mathrm{I}$ photoiragments with nonzero relative kinetic energy. These clearly must come from unbound states of the neutral complex that have Franck-Condon overlap with the anion. Transitions to the continuum of the I state could certainly account for their observation. It is also possible that they were observing vibrational predissociation from the $v_{3}=1$ levels of the $X$ and 1 sfates.

To summarize, ZEKE spectroscopy of the $\mathrm{S}_{\mathrm{N}} 2$ intermediate $\mathrm{I}^{-} \cdot \mathrm{CH}_{3}$ has been used to probe the open shell I. $\mathrm{CH}_{3} \mathrm{l}$ van der Waals complex. The band corresponding to transitions to the $\mathbf{X}$ state of the neutral shows vibrational structure from progressions in two low frequency van der Waals modes of the complex. As only one of these is totally symmetric, this suggests that the neutral complex undergoes Jahn-Teller distortion, activating the second (degenerate) mode. Transitions to the I state of the neutral complex were not apparent in the anion ZEKE spectrum. This was attributed to a significantly larger $\mathrm{C}-1$ bond distance I state compared to the anion, resulting in transitions to the continuum levels of the I state. The II state is observed, but no vibrational structure is resolved, possibly due to spectral congestion. 


\section{Acknowledgments}

This research is supportet by the Air Force Office of Scientific Research under Grant No. F49620-9-4-1-0115 (DMN), anid by the National Science Foundation under Grant No. CHE9207894 (MAJ). 
1D. M. Cyr, M. G. Scarton, and M. A. Johnson, J. Chem. Phys. 99, 4869 (1993).

2D. M. Cyr, G. A. Bishea, M. G. Scarton, and M. A. Johnson, J. Chem. Phys. 97,5911 (1992).

s. S. Shaik, Acta. Chem. Scand. 44, 205 (1990).

4 W.-P.Hu and D. G. Truhlar, J. Phys. Chem. 98,1049 (1994).

5W. N. Olmsteau and J. I. Brauman, J. Am. Chem. Soc. 99, 4219 (1977).

6S. T. Granland and M. 'r. Bowers, J. Am. Chem. Soc. 116, 3875 (1994).

7R. A. J. Ohair, G. E. Davies, J. Hacaloglu, T. T. Dang, and V. M. Bierbaum, J. Am. Chem. Soc. 116, 3609 (1994.

8W. L. Hase and Y. J. Cho, J. Chem. Phys. 98, 8626 (1993).

9Z. Shi and R. ن. Boyd, J. Am. Chem. Soc. 112, 6789 (1990).

10w. A. Chupka, A. M. Woodward, S. D. Colson, and M. G. White, J. Chem. Phys. 82, 4273 (1985).

11Y. Zhao, C. C. Amold, and D. M. Neumark, J. Chem. Soc. Faraday Trans. 89, 1449 (19S3).

12Y. Zhao, G. Reiser, I. Yourshaw, C. C. Amold, and D. M. Neumark, J. Chem. Phys. 101 (1994, in press).

${ }^{19}$ T. N. Kitsopoulos, I. M. Waller, J. G. Loeser, and D. M. Neumarh, Chem. Phys. Lett. 159, 300 (1989); C. C. Arnold, Y. Zhao, T. N. Kitsopoulos, and D. M. Neumark, J. Chem. Phys. 97, 6121 (1992). 
14M. A. Johnson, W. C. Lineberger, in Techniques in Chemistry, Vol. 20, J. M. Farrar and W. H. Saunders, eds. Wiley, New York (1982) p. 591.

15K. Müller-Dethlełs, M. Sander, Schlag, E. W. Z. Naturforsch., Teil a 39, 1089 (1984); K. Müller-Dethlefs, M. Sander, and E. W. Schlag, Chem. Phys. Lett. 43, 291 (1984); K. MütlerDethlefs and E. W. Schlag, Annu. Rev. Phys. Chem. 42, 109 (1991).

${ }^{16}$ R. C. Dougherty, J. Dalton, and J. D. Roberts, Org. Mass Spec. 8, 81 (1974).

${ }^{17}$ A. D. Dickson, I. M. Mills, and B. Crawtord, Jr., J. Chem. Pr.re 27, 445 (1957).

18 We also tried to fit the spectrum using displacement along only one of the normal coordinates, with two modes contributing to the sequence band structure. However, peak positions could not be as well matched, and the level of congestion we could simulate in this manner did not approach the congestion that is observed.

${ }^{19} \mathrm{G}$. Reiser, I. Yourshaw, Y. Zhao, and D. M. Neumark (to be published).

20V. Aquilanti, R. Condori, and F. Pirani, J. Chem. Phys. 89, 6157 (1988); V. Aquilanti, R. Condori, D. Cappeiletti, V. Lorent, and F. Pirani, Chem. Phys. 145, 293 (1990); V. Aquilanti, D. Cappelletti, V. Lorent, E. Luzzatti, and F. Pirani, Chem. Phys. Lett. 192, 153 (1992).

${ }^{21} \mathrm{G}$. Herzberg, Molecular Spectra and Molecular Structure III. Electronic Spectra and Electronic Structure of Polyatomic Molecules, Van Nostrand, New York (1966) pp. 151-157.

22H. C. Longuet-Higgens, U. Opik, M. H. L. Price, and R. A. Sack, Proc. R. Soc. London Ser. A 1, 244 (1958); H. C. Longuet-Higgens, Adv. Spectrosc. 2, 429 (1961). 
Figure (11.1) Previously obtained PES [Ref. (2)] and ZEKE spectrum of $1^{-} \cdot \mathrm{CH}_{3}$. The PES shown in the upper panel was obtained using $4.66 \mathrm{eV}$ detachment energy. The center and lower panels show the ZEKE spectrum in the energy region of the transition to the ground and excited spin-orbit states, respectively, of the neutral complex.
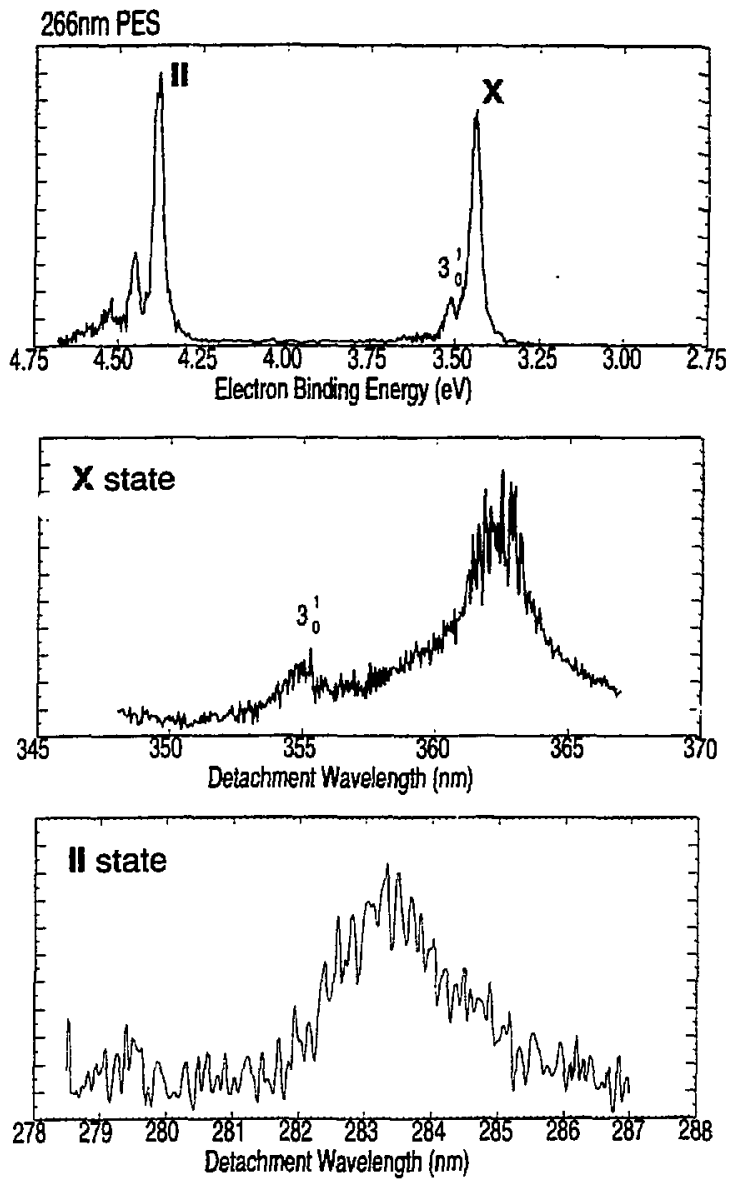
Figure (11.2) ZEKE spectrum of $1 . \mathrm{CH}_{3}$ I shown on an expanded scale near the origin of band $\mathrm{X}$ (dotted line) superimposed onto a spectral fit (solid line, see text).

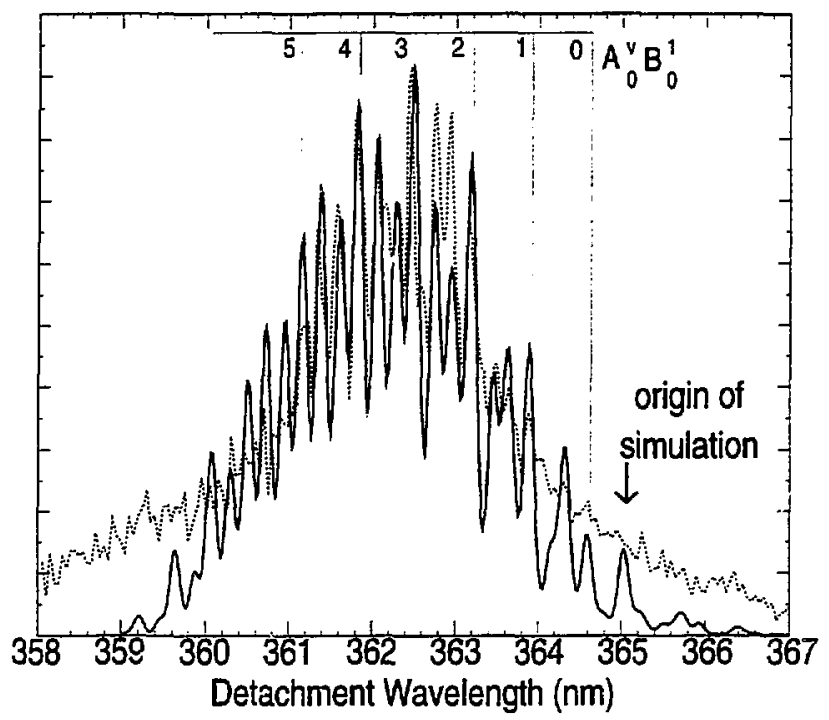


Figure (11.3) Schematic of the electronic states resulting from the electrostatic interaction between $\mathrm{I}^{-}$and I with $\mathrm{CH}_{3} \mathrm{l}$.
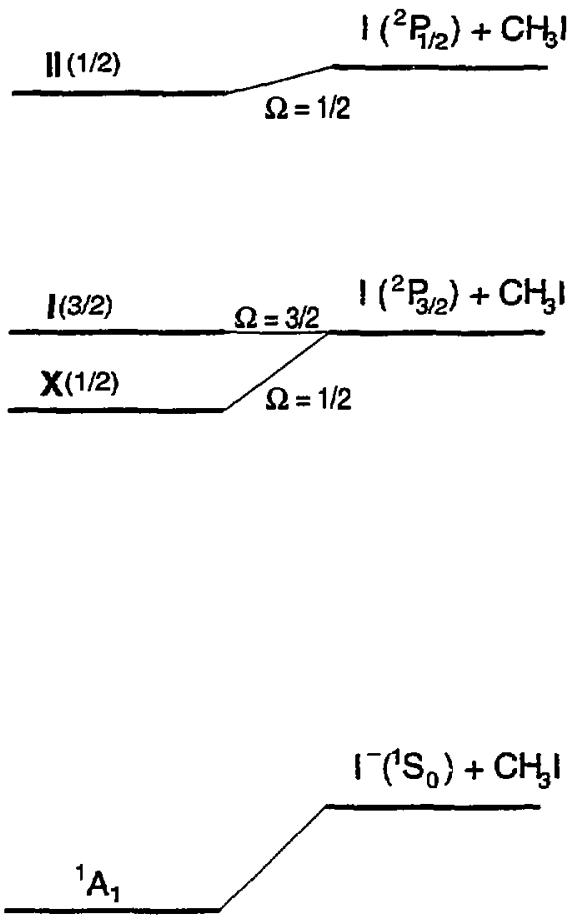

$R\left(1-\mathrm{CH}_{3} \mathrm{l}\right)$ 


\section{Appendix 1}

\section{Publications}

"Study of $\ln n_{2} P / / n_{2} P "$ and $I n F_{2} / n P_{2}-$ using negative ion threshold photodetachment spectroscopy," Caroline C. Amold and Danie! M. Neumark, accepted for publication, Canadian Joumal of Physics (1994).

"Negative Ion Threshold Photodetachment Spectroscopy of the I- $-\mathrm{CH}_{3} I \mathrm{~S}_{\mathrm{N}} 2$ Reaction Intermediate," Caroline C. Amold, Daniel M. Neumark, Donna M. Cyr, and Mask A. Johnson, accepted for publication, Joumal of Physical Chemistry (1994).

"Anion Photoelectron Spectroscopy of Small Indium Phosphide Clusters ( $\ln _{x} P_{y} ; x, y=1-4$ )," Cangshan Xu, Esther de Beer, Don W. Arnold, Caroline C. Amold, and Daniel $M$. Neumark, Joumal of Chemical Physics, 101, 5604 (1994).

"The study of small carbon and silicon clusters using negative ion photodetachment techniques," Caroline C. Amold and Daniel M. Neumark, Advances in Metal and Semiconductor Clusters, Vol. III, Edited by M. Duncan, JAl Press, Greenwich (1994).

"Threshold Photodetachment (ZEKE) Spectroscopy of $\mathrm{Si}_{3}-$, " Caroline C. Amold and Daniel M. Neumark, Joumal of Chemical Physics 100, 1797 (1994).

"Study of $\mathrm{Si}_{4}$ and $\mathrm{Si}_{4}{ }^{-}$using Threshold Phcto:Jetachment (ZEKE) Spectroscopy," Caroline C. Arnold and Daniel M. Neumark, Jounval of Chemical Physics 99, 3353 (1993).

"Study of $\mathrm{C}_{6}{ }^{-}$and $\mathrm{C}_{6}$ with Threshold Photodetachment Spectroscopy and Autodetachment Spectroscopy- Reply, "Caroline C. Amold and Daniel M. Neumark, Joumal of Chemical Physics 99, 1442 (1993).

"Reassignment of the $\mathrm{Si}_{2}-$ Photodetachment Spectra," Caroline C. Amold, Theofanis N. Kitsopoulos and Daniel M. Neumark. Journal of Chemical Physics 99, 766 (1993).

"Study of the $1 . \mathrm{CO}_{2}$ van der Waals Complex by Threshoid Photodetachment Spectroscopy of I$\mathrm{CO}_{2}$," Yuexing $\mathrm{X}$. Zhao, Caroline C. Arnold and Daniel M. Neumark, Journal of the Chemical Society- Faraday Transactions 89, 1449 (1993).

"Study of $\mathrm{C}_{6}$ " and $\mathrm{C}_{6}$ with Threshold Photodetachment Spectroscopy and Autodetachment Spectroscopy, " Caroline C. Amold, Yuexing X. Zhao, Theofanis N. Kitsopoulos and Daniel M. Neumark, Joumal of Chemical Physics 97, 6121 (1992).

"Dielectric Relaxation of Intercalated $\mathrm{Cd}_{2} \mathrm{P}_{2} \mathrm{~S}_{6}$ " Jeff A. Read, Caroline Chick and Anthony $\mathrm{H}$. Francis, Journal of Physical Chemistry 96, 2010 (1992). 
"Threshold Photodetachmeni Spectroscopy of $\mathrm{C}_{5}{ }^{-, "}$ Theofanis N. Kitsopoulos. Caroline J. Chick, Yuexing Zhao and Daniel M. iVtumark, Joumal of Chemical Physics 95, 5479 (1991).

"Study of the Low-lying Electronic States $\mathrm{St}_{\mathrm{Si}}$ and $\mathrm{Si}_{-}-$Using Negative lon Photodetachment Techniques," Theofanis N. Kitsopoulos, Caroline J. Chick, Yuexing Zhao and Daniel M. Neumark, Joumal of Chemical Physics 95, 1441 (1991).

"Vibrationalty Fiesolved Photoelectron Spectra of $\mathrm{Si}_{3}{ }^{-}$and $\mathrm{Si}_{4}{ }^{-}, "$ Theofanis N. Kitsopoulos, Caroline J. Chick, Alexandra Weaver and Daniel M. Neumark, Journal of Chemical Physics 93, 6108 (1990).

\section{Submitted for publication or in preparation}

"Study of small gallium arsenide clusters using negative ion photodetachment lechniques," Caroline C. Amold, Cangshan Xu, Gordon R. Burton, and Daniel M. Neumark, in preparation.

"Study of the low-lying states of Ge2- and Ge2 using negative ion ZF:KE spectroscopy," Carivine C. Amold, Cangshan Xu, Gordon R. Burton, and Daniel M. Neumark, in preparation.

"Study of the ArBr", Arl", and $\mathrm{Krl}^{-}$Anions and their Corresponding Neutral van der Waals Complexes by Anion Zero Electron Kinetic Energy Spectroscopy," Yuexing Zhao, Georg Reiser, Ivan Yourshaw, Caroline C. Amald, and Daniel M. Neumark, submitted to Journal of Chemical Physics, May, 1994. 


\title{
Appendix II
}

\section{Jahn-Teller interaction code (Fortran)}

\author{
Program jtchick
}

c This program, written by ccarnold 26 May 93, sets up a matrix for

c a degenerate $\mathrm{D} 3 \mathrm{~h}$ state (e.g., Si3-) experiencing Jahn-Teller

c distortion. The Matrix, all of the eigenvalues and eigenfunctions

$c$ are put into fort.9, and a shortened version is in fort.8. The

$c$ immediate output gives the matrix elements individually.

c

c format using $£ 77$-o jtchickl.f -o jtchickl -lnagd

$c$ ndim is $2 *(n+0.5(n-1))$ where $n$ is max vib quantum number

Parameter (ndim $=420$ )

implicit double precision $(a-h, 0-z)$

double precision evals (ndim), evecs (ndim, ndim)

double precision wk(ndim)

real*8 PROT (ndim, ndim)

write $(9, *)$ 'Chick $j-t$ coupling code output'

write $(8, *)$ 'chick short jt output'

write $(8, *)$ 'Erom jtchick. $\mathbf{f}^{\prime}$

write $(9, *), '$

write $(8, *)$ '"

$c$ puppy is the linear coupling constant, $k$, as in $k r e x p$ ( $i * p h i$ )

$c \mathrm{~g}$ is the quadratic coupling constant, as in $g\left(x^{\wedge} 2\right) \exp (i * 2 p h i)$

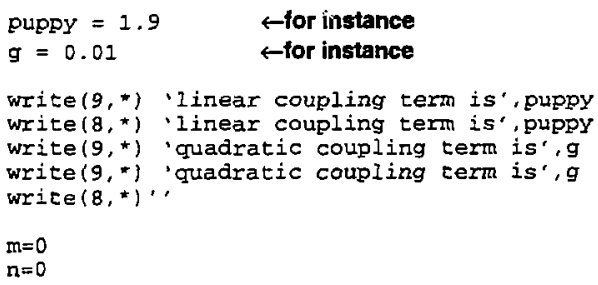

c I is the viorational quantum, $k$ is the ang mom quantum, and $i i$ is

$c$ the sign of the wv fn of the ket. ie, $i, k$, ii is $<n \mathrm{~m}+/-1$ in

$c$ the notation of Longuet-higgens

$c$ aj is the half quantum number 


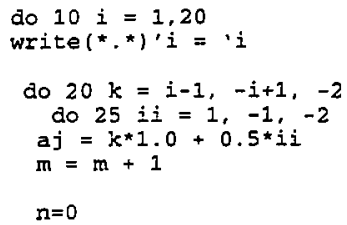

$c j$ is the vibrational quantu, 1 is the ang mom quantum, and $i$ is $c$ the sign of the wrfn of the bra. ie, j, $1, j j$ is $\mid n m+/ \rightarrow$

c bj is the half-quantum number

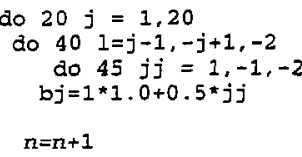

c write $(*, *) ' m=\prime, m^{\prime} n=\prime, n$

if $((i . e \cdot j)$. and. (1.eq. $(x+1)$.and. $(a j \cdot e q . b j))$ then $\operatorname{PROT}(m, \Omega)=i \star 1.0$ endif

c These two terms set up linear coupling

if $((j . e q .(i+1))$.and. $(1$. eq. $(k+1))$.and. $(a j \cdot e q . b j))$ then PROT $(m, n)=$ puppy ${ }^{\star} \operatorname{sqrt}(0.5 *(i+k+1) * 1.0)$ endif

if $((i . e q \cdot(j-1))$. and. $(k \cdot e q \cdot(1+1))$. and. $(a j \cdot e q \cdot b j))$ then $\operatorname{PROT}(m, n)=$ puppy* sqrt $(0.5 *(j-1-1) * 1.0)$ endif

c These three terms set up the quadratic coupling if $((j . e q .(i+2))$. and. (bj.eq. $(a j+3.0)))$ then $\operatorname{PROT}(m, n\}=0.25 * g * \operatorname{sqrt}(\{i+k+1) *(i+k+3)\} * 1,0)$ endif

if $(\{i \cdot$ eq. $(j-2))$. and. $(a j \cdot e q \cdot(b j+3.0)))$ then endif PROT $(m, n)=0.25^{*} g^{\star} \operatorname{sgrt}((j-1-1) *(j-1-3) * 1,0)$

if $((j . e q . i)$. and. (bj.eq. $(a j+3.0))$ then

PROT $(m, n)=0.5^{*} g^{*} \operatorname{sgrt}((i+k+1) *(i-k-1) * 1.0)$ endif

$\operatorname{PRUTT}(n, m)=\operatorname{PROT}(m, n)$

45 zontinue

40 continue

30 continue

25 continue

20 continue

10 continue 